\title{
Ground-Water Withdrawals, Water-Level Changes, Land-Surface Subsidence, and Ground-Water Quality in Fort Bend County, Texas, 1969-87
}

By Glenn L. Locke

U.S. GEOLOGICAL SURVEY

Water-Resources Investigations Report 90-4012

Prepared in cooperation with

FORT BEND COUNTY

Austin. Texas 
DEPARTMENT OF THE INTERIOR

MANUEL LUJAN, JR., Secretary

U.S. GEOLOGICAL SURVEY

Dallas L. Peck, Director

For additional information write to:

District Chief

U.S. Geological Survey

8011 Cameron Rd.

Austin, TX 78753
Copies of this report can be purchased from:

U.S. Geological Survey

Books and Open-File Reports Section Federal Center, B1dg. 810

Box 25425

Denver, C0 80225 
Abstract-1

Introduction-10.3

Location and extent of the area- 3

Wel1-numbering system-1... 3

Previous investigations-1... 6

Hydrogeology

Development of ground water-1... 8

Ground-water withdrawals- 10

Water-level changes-1.

Water-level changes in the Houston district-...... 14

Water-level changes in Fort Bend County-..... 14

Upper unit of the Chicot aquifer-

Lower unit of the Chicot aquifer

Evangeline aquifer- 19

Land-surface subsidence-1. 19

Thickness of clay-..... 24

Relation between thickness of clay, water-level

declines, and subsidence-1..... 25

Areas most susceptible to land-surface subsidence-1................. 33

Quality of ground water. 33

Summary and conclusions-1... 34

References cited-1 37

Supplemental data-1... 40

\section{ILLUSTRATIONS}

Figure 1. Map showing location of Fort Bend County- 4

2. Map showing location of wells

3. Map showing location of principal areas of ground-water withdrawals and average withdrawal rates in 1986 in the Houston district and northern Fort Bend County-... 7

4. Graph showing ground-water withdrawals in Fort Bend County, 1969-86-11

5. Graph showing ground-water withdrawals in the Houston and Katy areas of the Houston district and in the northeast area and Katy area of Fort Bend County, 1930-86

6. Approximate altitude of water levels in wells screened in the lower unit of the Chicot aquifer, spring 1987-.... 15

7. Approximate altitude of water levels in wells screened in the Evangeline aquifer, spring 1987-........ 16

8. Approximate change in water levels in wells screened in the lower unit of the Chicot aquifer, 1968-69 to 1987-1. 
Figure 9. Hydrographs showing water levels in wells screened

in the lower unit of the Chicot aquifer in the Katy

area and southwestern part of Fort Bend County-.... 20

10. Hydrographs showing water levels in wells screened in

the lower unit of the Chicot aquifer in the

northeastern part of Fort Bend County

11. Map showing approximate change in water levels in

wells screened in the Evangeline aquifer,

1968-69 to 1987

12. Hydrographs showing water levels in wells screened in

the Evangeline aquifer.

13-16. Maps showing:

13. Approximate cumulative thickness of clay beds

in the Chicot aquifer-... 26

14. Approximate cumulative thickness of clay beds

in the Evangeline aquifer... 27

15. Approximate cumulative thickness of clay beds

in the Chicot and Evangeline aquifers.............. 28

16. Location of compaction monitor sites in the

Houston district and average specific-unit

compaction-... 30

17. Graph showing depth to water below land surface at well

JY-65-10-702 and subsidence of a bench mark at Katy-

TABLES

Table 1. Correlation of geologic and and hydrologic units

2. Records of selected wells in Fort Bend County-............ 42

3. Drillers' logs of selected wells in

Fort Bend County, 1969-87-2 72

4. Water levels in selected wells in

Fort Bend County, 1969-87-131

5. Average specific-unit compaction values-_...

6. Chemical analyses of water from wells

in Fort Bend County, 1969-87-144

7. Statistical summary of the concentrations of major

dissolved ions, and the values of specific conductance,

hardness, and sodium-adsorption ratio- 


\section{CONVERSION FACTORS}

Factors for converting inch-pound units to metric (International System) units are given in the following table:

\begin{tabular}{|c|c|c|}
\hline Multiply inch-pound unit & By & To obtain metric units \\
\hline inch (in.) & 25.4 & millimeter (mm) \\
\hline acre & 0.4047 & hectare \\
\hline foot $(\mathrm{ft})$ & 0.3048 & meter (m) \\
\hline per foot $(f t-1)$ & 3.2808 & per meter $\left(m^{-1}\right)$ \\
\hline foot per year $(\mathrm{ft} / \mathrm{yr})$ & 0.3048 & meter per year $(\mathrm{m} / \mathrm{yr})$ \\
\hline gallon per minute $(\mathrm{gal} / \mathrm{min})$ & 0.06308 & liter per second $(L / s)$ \\
\hline mile $(m i)$ & 1.609 & kilometer $(\mathrm{km})$ \\
\hline $\begin{array}{l}\text { million gallons per day } \\
\text { (Mgal/d) }\end{array}$ & 0.04381 & $\begin{array}{l}\text { cubic meter per second } \\
\left(\mathrm{m}^{3} / \mathrm{s}\right)\end{array}$ \\
\hline square mile $\left(\mathrm{mi}^{2}\right)$ & 2.590 & square kilometer $\left(\mathrm{km}^{2}\right)$ \\
\hline
\end{tabular}

Milligrams per liter $(\mathrm{mg} / \mathrm{L})$ : A unit expressing the concentration of a chemical constituent in solution as weight (milligrams) of solute per unit volume (1iter) of water. One $\mathrm{mg} / \mathrm{L}$ equals 1,000 micrograms per liter.

Sea level: In this report "sea level" refers to the National Geodetic Vertical Datum of 1929 (NGVD of 1929)--a geodetic datum derived from a general adjustment of the first-order level nets of both the United States and Canada, formerly called "Sea Level Datum of 1929." 


\title{
GROUND-WATER WITHDRAWALS, WATER-LEVEL CHANGES, \\ LAND-SURFACE SUBSIDENCE, AND GROUND-WATER QUALITY
}

IN FORT BEND COUNTY, TEXAS, 1969-87

\author{
By \\ Glenn L. Locke
}

\section{ABSTRACT}

Fort Bend County, which has one of the fastest growing populations of all counties in the United States, is dependent entirely on ground water for public supply. Since 1969, at least 90 large-capacity wells have been drilled, of which 57 were public supply wells, 23 were irrigation wells, and 10 were industrial wells. All but seven of the new public-supply wells are located in the northeastern part of the county.

The withdrawal of ground water in Fort Bend County increased from 56 million gallons per day in 1969 to 72 million gallons per day in 1982, and then decreased to 53 million gallons per day in 1986. Withdrawals for public supply increased from 4 million gallons per day in 1969 to 28 million gallons per day in 1986. Withdrawals for irrigation averaged 38 million gallons per day during 1969-82 and in 1984, but averaged 20 million gallons per day in 1983, 1985, and 1986. Withdrawals for industrial use declined from 13 million gallons per day in 1969 to 5 million gallons per day in 1986.

Water levels in wells screened in the upper unit of the Chicot aquifer generally fluctuated less than 4 feet between 1968-69 and 1987. During the same period, water-level declines in wells screened in the lower unit of the Chicot aquifer ranged from less than 10 feet in most of the western part of the county to 100 feet in the northeastern corner. In the southwestern part of the county, the decline was less than 20 feet. Hydrographs of wells completed in the lower unit of the Chicot showed that water levels continued to decline from 1969 to the early 1980's. The hydrographs of wells located outside of the northeast area generally show a stabilization of water levels corresponding to the reduction in withdrawals after 1982 in Fort Bend County and in the neighboring Houston metropolitan area to the northeast.

Withdrawals from the Evangeline aquifer increased from 15 percent of the total in 1969 to 50 percent in 1986. Water-level declines in wells screened in the Evangeline aquifer during the same period ranged from less than 25 feet in the northwestern part of the county to 125 feet in the northeastern part. 
In the southwestern one-quarter of the county, the decline was less than 50 feet.

Declines in the potentiometric surfaces of the aquifers have caused compaction of clay resulting in land-surface subsidence. Between 1906 and 1978, about 65 percent of the county subsided more than 0.5 foot. Studies in the Houston area have shown that most clay compaction occurs in the Chicot aquifer and that the compressibility of the clays increases towards the coast. The total thickness of clays in the Chicot aquifer increases from less than 150 feet in the northwestern part of Fort Bend County to more than 350 feet along the eastern border. The total thickness of clay in the Evangeline aquifer increases from an average of about 700 feet in the northwest to about 1,100 feet in the east. The northeastern part of Fort Bend County is most susceptible to future subsidence because it is the area where the largest water-level declines have occurred and where the thickest, most compressible clays in the county are found.

The concentrations of dissolved solids in water from wells in Fort Bend County have not changed appreciably from 1969 to 1987 . The median concentrations of dissolved solids are 475 milligrams per liter in water from the upper unit of the Chicot aquifer, 337 milligrams per liter in water from the lower unit of the Chicot aquifer, and 307 milligrams per liter in water from the Evangeline aquifer. 


\section{INTRODUCTION}

The population of Fort Bend County in southeastern Texas (fig. 1) is one of the fastest growing in the United States, increasing from 52,314 in 1970 (U.S. Bureau of the Census, 1973) to 179,732 in 1986 (Texas A\&M University, 1986). Ground water has been developed extensively to meet the needs of the growing population of the county and the city of Houston in adjacent Harris County. An investigation of the ground-water resources of Fort Bend County was conducted by the U.S. Geological Survey during 1968-69 (Wesselman, 1972), but little data has been collected since.

This report documents ground-water withdrawals and changes in water levels and water quality in Fort Bend County since the 1968-69 investigation by Wesselman (1972), and delineates areas susceptible to land-surface subsidence. The investigation was a cooperative project of the U.S. Geological Survey and Fort Bend County.

The investigation included: (1) An inventory of industrial, public supply, and irrigation wells, and a representative number of domestic and livestock wells; (2) an inventory of the withdrawal of ground water for industrial, public supply, and irrigation uses; (3) compilation and analysis of measurements of water levels in wells since 1969; (4) determination of clay thickness based on electric logs of wells and test holes in the Chicot and Evangeline aquifers; (5) delineation of the area most susceptible to landsurface subsidence on the basis of clay thickness, water-level declines, and studies from adjacent areas; and (6) analyses of water samples to determine changes in the chemical quality of the water.

\section{Location and Extent of the Area}

Fort Bend County, located in southeast Texas, is bordered by Harris County on the northeast, Brazoria County on the southeast, Wharton County on the southwest, and Austin and Waller Counties on the northwest (fig. 1). The county has an area of $862 \mathrm{mi}^{2}$. The topography varies from level to rolling prairie. The altitude ranges from $50 \mathrm{ft}$ above sea level in the southeast to $200 \mathrm{ft}$ above sea level in the northwest. The county is drained by the Brazos and San Bernard Rivers. The climate is subtropical.

\section{We11-Numbering System}

The location of wells in Fort Bend County is shown in figure 2. The well-numbering system used in this report is the system adopted by the Texas Water Development Board for use throughout the State. In this system, each 1degree quadrangle in the State is given a number consisting of two digits from 01 to 89. These are the first two digits in the well number. Each 1-degree 


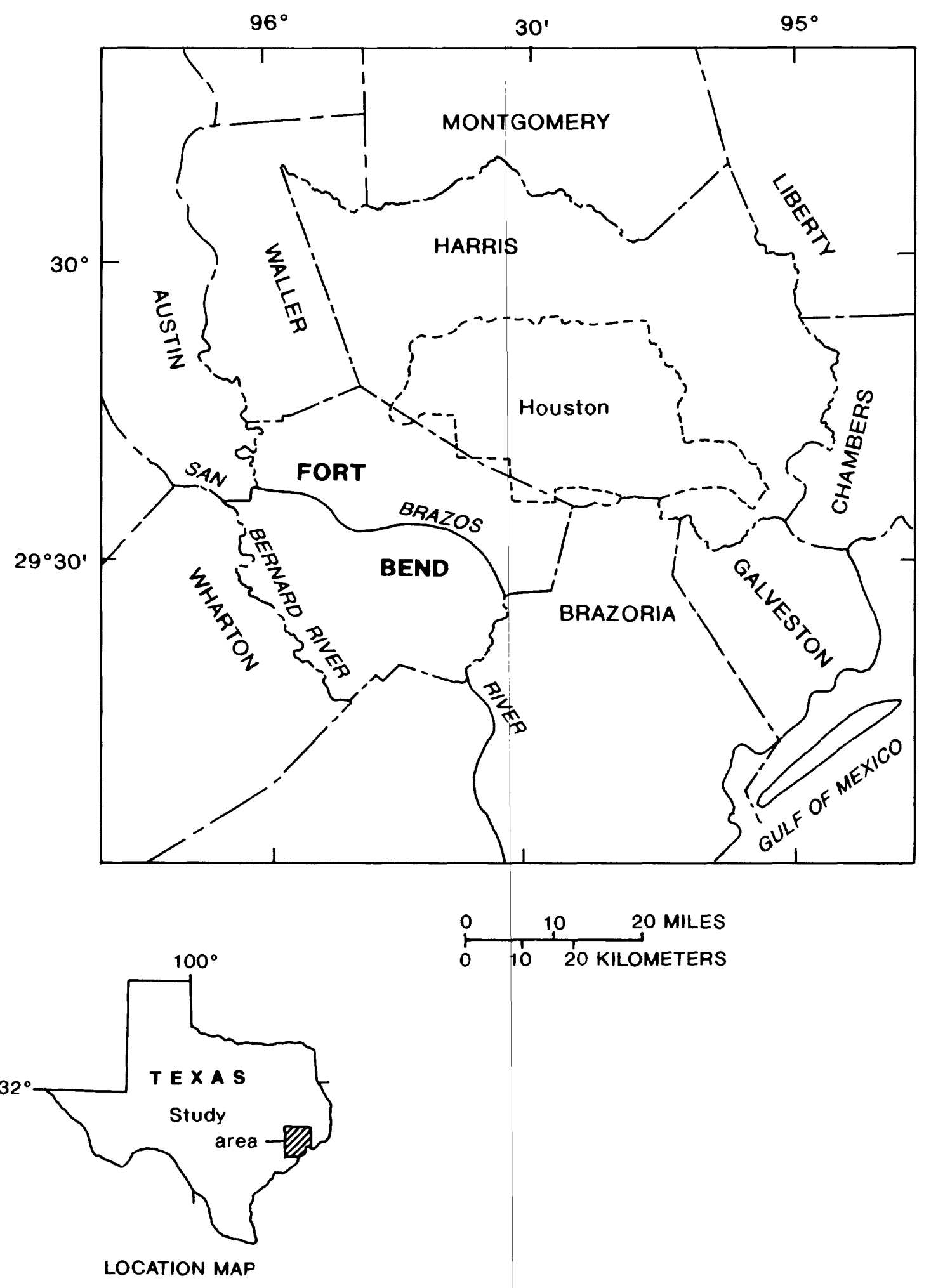

Figure 1.--Location of Fort Bend County. 


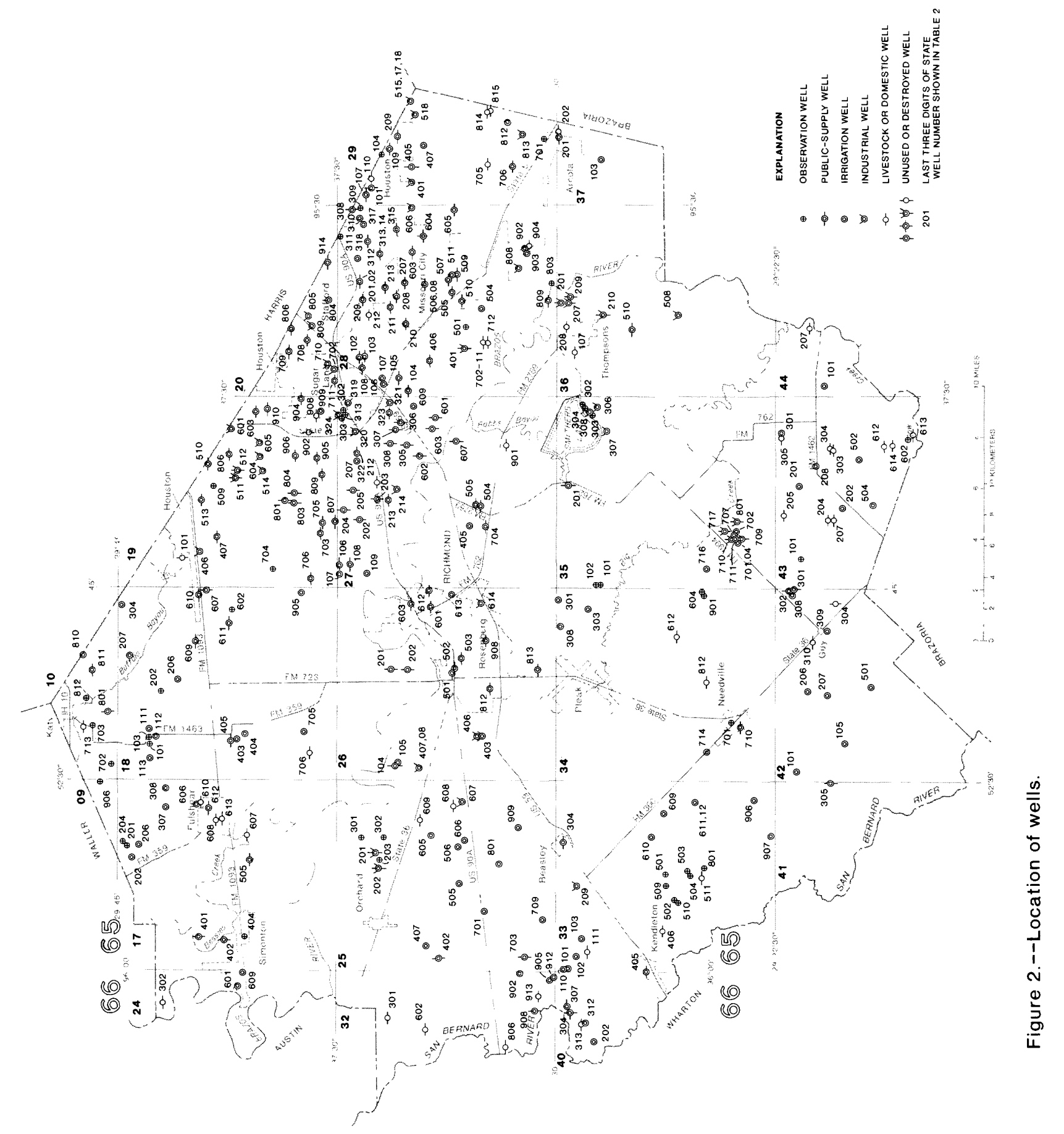


quadrangle is divided into 7-1/2-minute quadrangles which are given a 2-digit number from 01 to 64 . These are the third and fourth digits of the well number. Each 7-1/2-minute quadrangle is divided into 2-1/2-minute quadrangles and given single-digit numbers from 1 to 9 . This is the fifth digit of the well number. Finally, each well within a 2-1/2-minute quadrangle is given a two-digit number in the order in which it was inventoried, starting with 01. These are the last two digits of the well number. In addition to the 7-digit well number, a 2-letter prefix is used to identify the county. The prefix for Fort Bend County is JY. As an example, well JY-65-26-501 is located in Fort Bend County, in the 1-degree quadrangle 65 , in the 7-1/2-minute quadrangle 26, in the 2-1/2-minute quadrangle 5, and was the first well inventoried, 01 .

\section{Previous Investigations}

Wesselman (1972, p. 6) summarized the earlier ground-water investigations of Fort Bend County. Records of the large-capacity wells were presented by Naftel and others (1976), Ratzlaff and others (1983), and Williams and others (1986). These records of wells, drillers' logs, water-level measurements, and chemical analyses are incorporated in tables in the section "Supplemental Data" at the end of this report.

Several reports have described the ground-water hydrology of the Houston district (fig. 3) of which Fort Bend County is a part. The Houston district, as used in this report, includes all of Harris and Galveston Counties and parts of Chambers, Liberty, and Fort Bend Counties. Gabrysch (1972, 1980, and 1984), and Williams and Ranzau (1987) described the ground-water development of the district. Wood and Gabrysch (1965) constructed the first analog model of the ground-water system of the Houston district. Jorgensen (1975) updated the analog model. Meyer and Carr (1979) created a digital ground-water model of the Houston district. Gabrysch (1969 and 1982), and Gabrysch and Bonnet (1975) evaluated land-surface subsidence, Holzer and Gabrysch (1987) related fault creep to ground-water withdrawals, and Gabrysch (1977) presented a map of the area of recharge to the aquifers. A series of water-level change and water-level altitude maps have been prepared annually since 1977; the latest were prepared by Williams, Ranzau, and Lind (1987) and by Williams, Lind, and Coplin (1987).

Wilson (1967) reported on the ground-water resources of adjacent Austin and Waller Counties. Sandeen and Wesselman (1973) investigated the groundwater resources of Brazoria County. Loskot and others (1982) reported on ground-water resources of Wharton County.

\section{Hydrogeology}

The Chicot and Evangeline aquifers, as described by Wesselman (1972, p. 


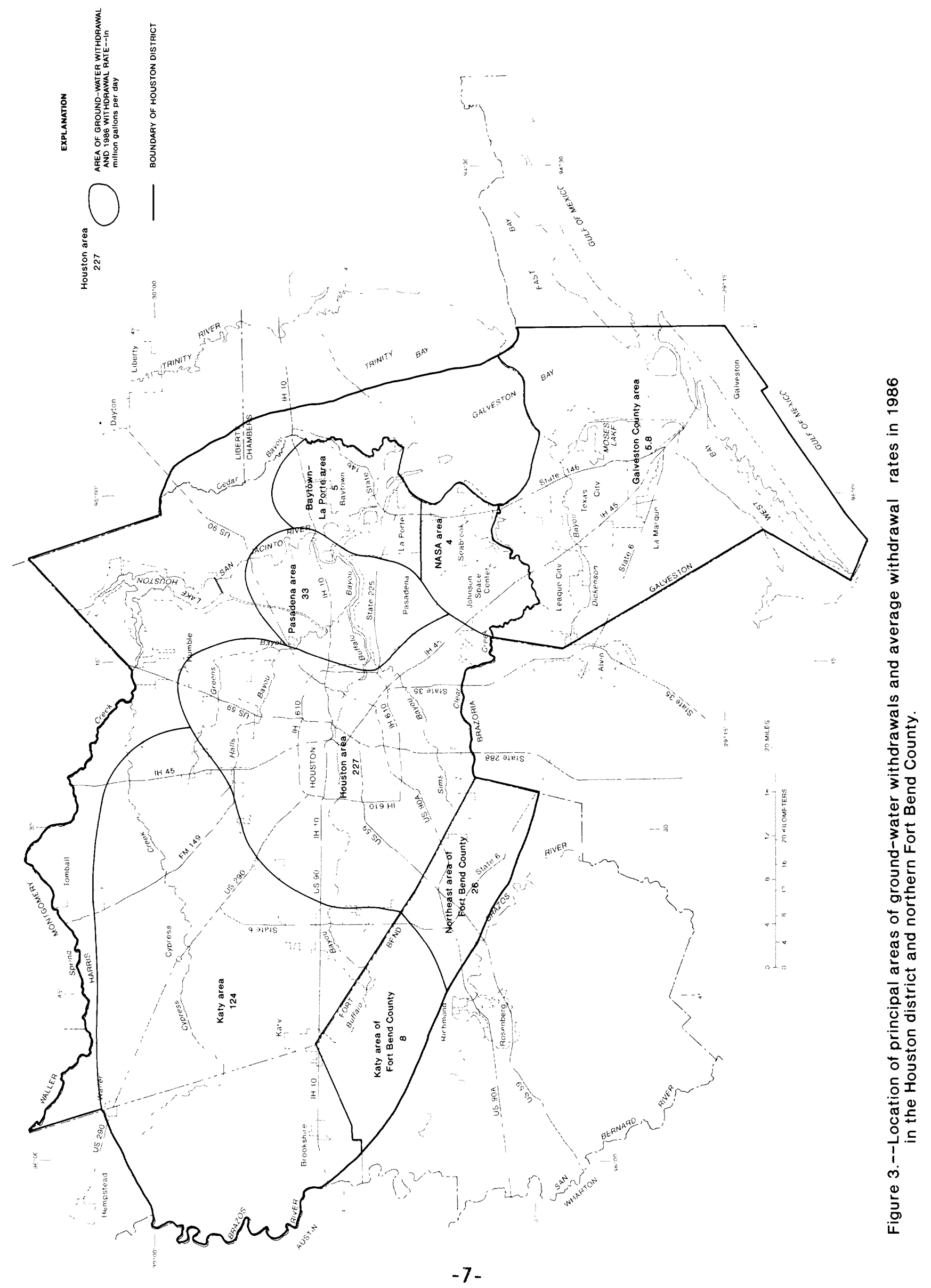


6-16) are the major freshwater aquifers in the county. The relation between these units and geologic units is shown in table 1 (Supplemental Data).

The Chicot aquifer consists of interbedded and discontinuous lenses of clay, silt, sand, and gravel from land surface to the top of the Evangeline aquifer. In the southeastern part of the county, the Chicot aquifer can be separated into an upper and lower unit by a clay layer, 200 to $300 \mathrm{ft}$ below 1 and surface (Wesselman, 1972, fig. 19). According to Jorgensen (1975, p. 10), in areas where the Chicot is undifferentiated, "Wells that are completed in the uppermost sand layers of the Chicot aquifer and that have water levels that are distinctly higher than water levels in wells completed in the underlying sand layers are considered to produce water from the upper unit."

Artesian conditions exist in most of the Chicot aquifer. Water-table conditions exist in the upper unit of the aquifer along major stream valleys where surficial sand deposits overlie shallow sands of the aquifer. In some outcrop areas, water-level declines have been great enough in some upper sands in the aquifer to cause a change from artesian to water-table conditions.

Because of their similar origin, the sediments of the Evangeline aquifer are much like the sediments of the Chicot aquifer, consisting of interbedded clay, silt, sand, and gravel. However, the Evangeline is distinguished from the Chicot by its finer grained sands, smaller sand to clay ratio, smaller hydraulic conductivity, and lower water levels.

\section{Development of Ground Water}

Since 1969, at least 90 large-capacity wells have been drilled in Fort Bend County (table 2 in Supplemental Data), more than doubling the number of large-capacity wells in the county. A large-capacity well for the purpose of this report is considered to be one with a discharge greater than $500 \mathrm{gal} / \mathrm{min}$. Fifty-seven wells were drilled for public supply, 23 wells for irrigation, and 10 wells for industrial supply ( 2 wells drilled by the Jefferson Lake Sulphur Company have been unused since 1984).

In 1968, 12 large-capacity and three 10-inch diameter public-supply wells had been drilled to an average depth of $960 \mathrm{ft}$. Eight of the wells were located north of the Brazos River and seven south of the river. In 1987, 69 1 arge-capacity public-supply wells were in use (average depth, 1,130 ft), 61 of which were located north of the Brazos River, primarily in the northeastern part of the county. Of the 57 wells drilled since 1969, 50 are in the northeastern part of the county and supply water to large housing subdivisions built and expanded since 1969. The average depth and yield of these 50 wells are $1,180 \mathrm{ft}$ and $1,460 \mathrm{gal} / \mathrm{min}$. The typical public-supply well drilled in the northeastern part is screened a total of $260 \mathrm{ft}$ between 700 and $1,150 \mathrm{ft}$ below 
land surface. About 80 percent of the screened sections are in the Evangeline aquifer.

In 1968, 36 large-capacity rice-irrigation wells had been drilled in the county (Wesselman, 1972, table 4). In addition, Wesselman listed 32 riceirrigation wells that had possible discharges greater than $500 \mathrm{gal} / \mathrm{min}$. The average depth of these 68 wells was $640 \mathrm{ft} ; 43$ of these wells (63 percent) were located north of the Brazos River, mostly in the Katy area. In 1987, 63 rice-irrigation wells (average depth $670 \mathrm{ft}$ ) were in use, but only 27 wells (43 percent) were located in the Katy area. Eighteen of the 23 large-capacity wells drilled for irrigation since 1969 are used for rice irrigation. The average depth and yield of the rice-irrigation wells are $735 \mathrm{ft}$ and 3,320 gal/min. The typical irrigation well in use in 1987 has a slotted casing for a total of $500 \mathrm{ft}$ between $240 \mathrm{ft}$ and $750 \mathrm{ft}$ below 1 and surface. About $75 \mathrm{per}$ cent of the slotted casing is open to the Chicot aquifer.

Wesselman (1972, table 4) reported 18 large-capacity industrial wells in Fort Bend County. Twenty-three additional wells had probable discharges greater than $500 \mathrm{gal} / \mathrm{min}$ or were used continuously in sulfur or salt mining. The average depth of the 41 industrial wells was $520 \mathrm{ft}$. Twenty-two of these wells were used in sulfur mining, which has since stopped. In 1987, 17 largecapacity industrial wells, which had an average depth of $825 \mathrm{ft}$, were in use. The 10 industrial wells drilled since 1969 have an average depth of $725 \mathrm{ft}$ and an average yield of 1,180 gal/min. The typical industrial well in use in 1987 is screened a total of $185 \mathrm{ft}$ between $425 \mathrm{ft}$ and $720 \mathrm{ft}$ below land surface. About 75 percent of the screened section is open to the Chicot aquifer.

Only a few of the hundreds of small domestic wells in the county were inventoried. According to U.S. Bureau of the Census data, the number of households served by a domestic well (a well supplying water to less than 6 houses) in 1970 was 5,964, or 40 percent of the 15,009 households in the county (U.S. Bureau of the Census, 1972). Most of the households, 59 percent, were served by a public-supply system. One percent of the households had sources of water other than ground water (creeks, rivers, cisterns, etc). In 1980, the number of households served by a domestic well increased to 7,379, but this represented only 17 percent of the 43,092 households in the county (U.S. Bureau of the Census, 1983). The number of households served by a public-supply system increased to 83 percent.

Drillers' logs of some of the recentiy drilled wells are presented in table 3 in Supplemental Data. Water levels in selected wells are shown in table 4 in Supplemental Data. 


\section{GROUND-WATER WITHDRAWALS}

Average daily withdrawal of ground water in Fort Bend County for public supply, irrigation, and industrial use is shown in figure 4 . The amount of ground-water withdrawn for public supply and industrial use was obtained from the Texas Water Development Board (written commun., 1987). Estimates of withdrawal for irrigation use are based primarily on amount of water applied per acre for rice irrigation and on the acreage planted. The average amount of ground water applied per acre of rice was reported by Gabrysch (1980, 1982), Williams and Ranzau (1987), and J.F. Williams (U.S. Geological Survey, written commun., 1987). The number of acres of rice planted was obtained from the U.S. Department of Agriculture (written commun., 1987).

The total amount of ground water withdrawn for all uses in 1986 (53 $\mathrm{Mgal} / \mathrm{d}$ ) was about the same as in 1969 (56 Mgal/d). Until 1982, the principal use of ground water in the county was for irrigation, and the total withdrawals have paralleled the variations in irrigation withdrawals.

Use of ground water for public supply has steadily increased from about 4 $\mathrm{Mgal} / \mathrm{d}$ in 1969 to $28 \mathrm{Mgal} / \mathrm{d}$ in 1986. Most of this increase has been caused by the urbanization of the northeastern part 1 of the county. Withdrawals for public supply in this area were about $2 \mathrm{Mgal} / \mathrm{d}$ in 1969 and $22 \mathrm{Mgal} / \mathrm{d}$ in 1986. About 50 percent of the total public-supply withdrawal of $4 \mathrm{Mgal} / \mathrm{d}$ in 1969 came from the Evangeline aquifer. In 1986, about 80 percent of the total public-supply withdrawal of $28 \mathrm{Mgal} / \mathrm{d}$ came from the Evangeline aquifer.

Ground-water withdrawals for irrigation averaged 38 Mgal/d during 1969-82 and 1984, but only averaged $20 \mathrm{Mgal} / \mathrm{d}$ in 1983, 1985, and 1986. Withdrawal for irrigation is directly related to number of acres planted. Rice is the principal irrigated crop in Fort Bend County. In 1969, 1974, 1979, and 1984, about 90 percent of the irrigated 1 and in the county was planted in rice (Texas Water Development Board, 1975, 1986). In these years, ground water was used to irrigate about 70 percent of the 1 and $p l$ anted in rice. The number of acres irrigated with surface water was obtained from the Brazos River Authority (oral commun., 1987) and the Richmond Canal Company (oral commun., 1987). The acreage planted in rice and irrigated with ground water since 1969 has ranged from 15,000 to 19,000 acres, with an average of 17,500 acres, excluding 1983, 1985, and 1986 when the acreage averaged 8,700 acres.

Water withdrawn for irrigation is principally from the Chicot aquifer. In 1969, about 90 percent of the $39 \mathrm{Mgal} / \mathrm{d}$ withdrawn for irrigation was from the Chicot; in 1986, about 85 percent of the $19 \mathrm{Mgal} / \mathrm{d}$ of water came from the chicot aquifer. The remainder of the water was withdrawn from the Evangeline aquifer. Most of the water withdrawn is in the northwestern and southern parts of the county. 


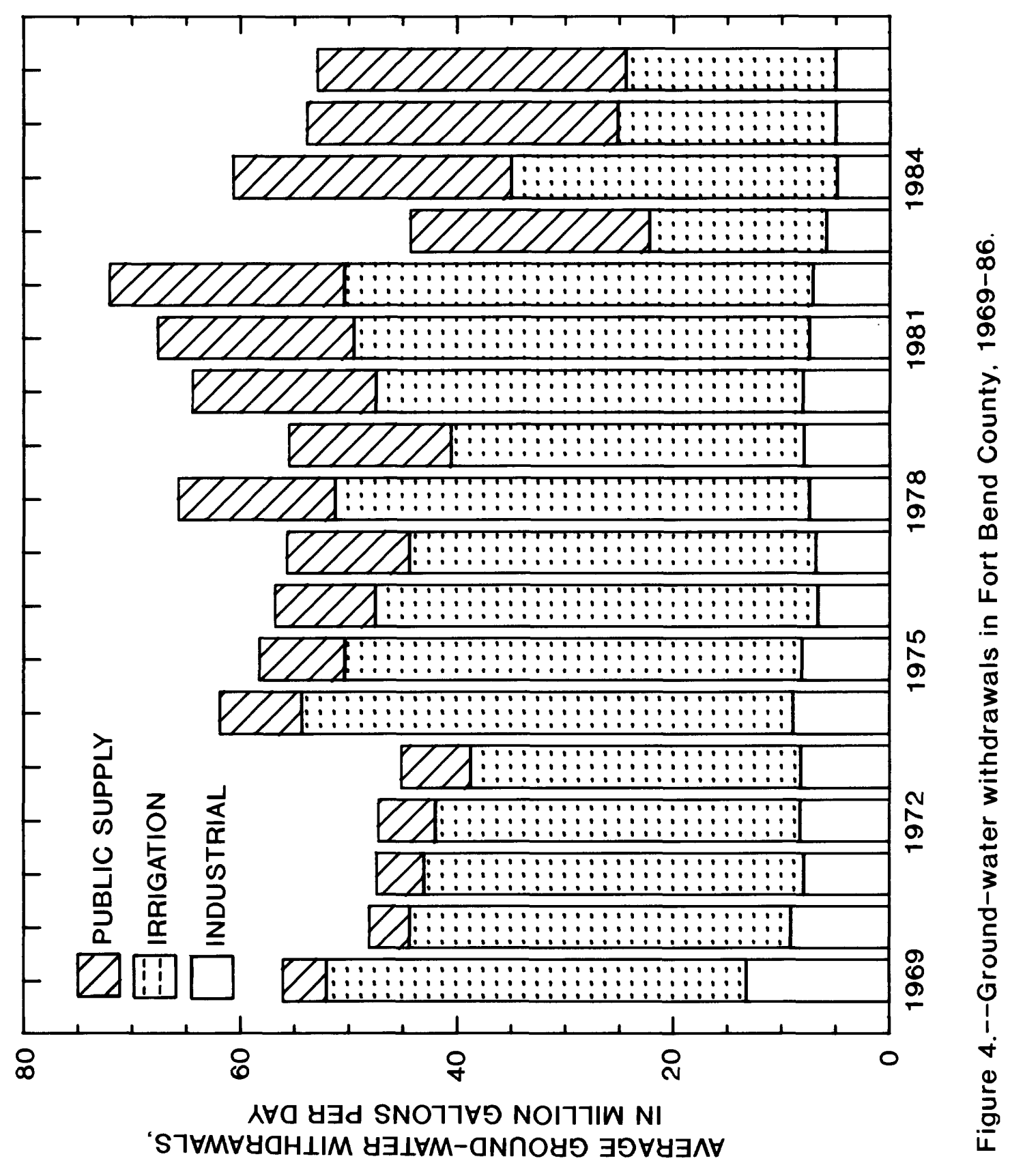


Withdrawal for industrial use was about $13 \mathrm{Mgal} / \mathrm{d}$ in 1969 but only 5 $\mathrm{Mgal} / \mathrm{d}$ in 1986. Three sulfur-mining operations used $10 \mathrm{Mgal} / \mathrm{d}$ of ground water in 1969. Two of the mines closed in 1970, and the other mine closed in 1984. In 1969, 90 percent of the water used for industrial purposes came from the Chicot aquifer. In 1986, 70 percent of the water came from the Chicot aquifer. Since 1969, surface water has supplied an estimated 85 percent of the water requirements for industrial purposes.

Water from the Evangeline aquifer accounted for more of the total withdrawals in 1986 than in 1969 because of the increase in public-supply withdrawal from the Evangeline aquifer and the decrease in industrial and irrigation withdrawal from the Chicot aquifer. In 1969, about 15 percent of the $56 \mathrm{Mgal} / \mathrm{d}$ of ground-water withdrawn in the county came from the Evangeline aquifer. In 1986, about 50 percent of the $53 \mathrm{Mgal} / \mathrm{d}$ withdrawn was from the Evangeline aquifer.

Ground-water withdrawals in the northeast and Katy areas of rort Bend County and the adjacent Katy and Houston areas of the Houston district are shown in figure 5 . Historically, variations in withdrawals in the Katy areas, have paralleled each other, whereas the withdrawal pattern has differed in the Houston and northeast areas. From the early 1960's to 1986, ground-water withdrawals in the Katy area of the Houston district averaged about 140 $\mathrm{Mgal} / \mathrm{d}$. In the Katy area of Fort Bend County, withdrawals averaged about 20 Mgal/d during the same period, except in 1983, 1985, and 1986, when withdrawals decreased to an average of about $8 \mathrm{Mgal} / \mathrm{d}$ because of decreases in rice acreage planting. Increases in public-supply withdrawals in the Katy area of the Houston district have offset the more than 50-percent decrease in irrigation withdrawals in those years. Public-supply and industrial withdrawals have made up less than 5 percent of the withdrawals in the Katy area of Fort Bend County.

In the Houston area of the Houston district, ground-water withdrawals nearly doubled from about $100 \mathrm{Mgal} / \mathrm{d}$ in the early 1960's to about $195 \mathrm{Mgal} / \mathrm{d}$ in the early 1970's. Withdrawals increased from about $185 \mathrm{Mgal} / \mathrm{d}$ in 1975 to about $260 \mathrm{Mgal} / \mathrm{d}$ in 1982, but decreased to about $230 \mathrm{Mgal}$ in 1986. About 95 percent of the withdrawals have been for public supply. Withdrawals in the northeastern part of Fort Bend County have steadily increased from about 2 $\mathrm{Mgal} / \mathrm{d}$ in the early 1960's to about $26 \mathrm{Mgal} / \mathrm{d}$ in 1986, resulting primarily from increases in public-supply withdrawals.

\section{WATER-LEVEL CHANGES}

The withdrawal of water from an artesian aquifer causes an immediate lowering of the potentiometric surface in the vicinity of the well--a cone of depression. The potentiometric surfaces of both the Chicot and Evangeline 

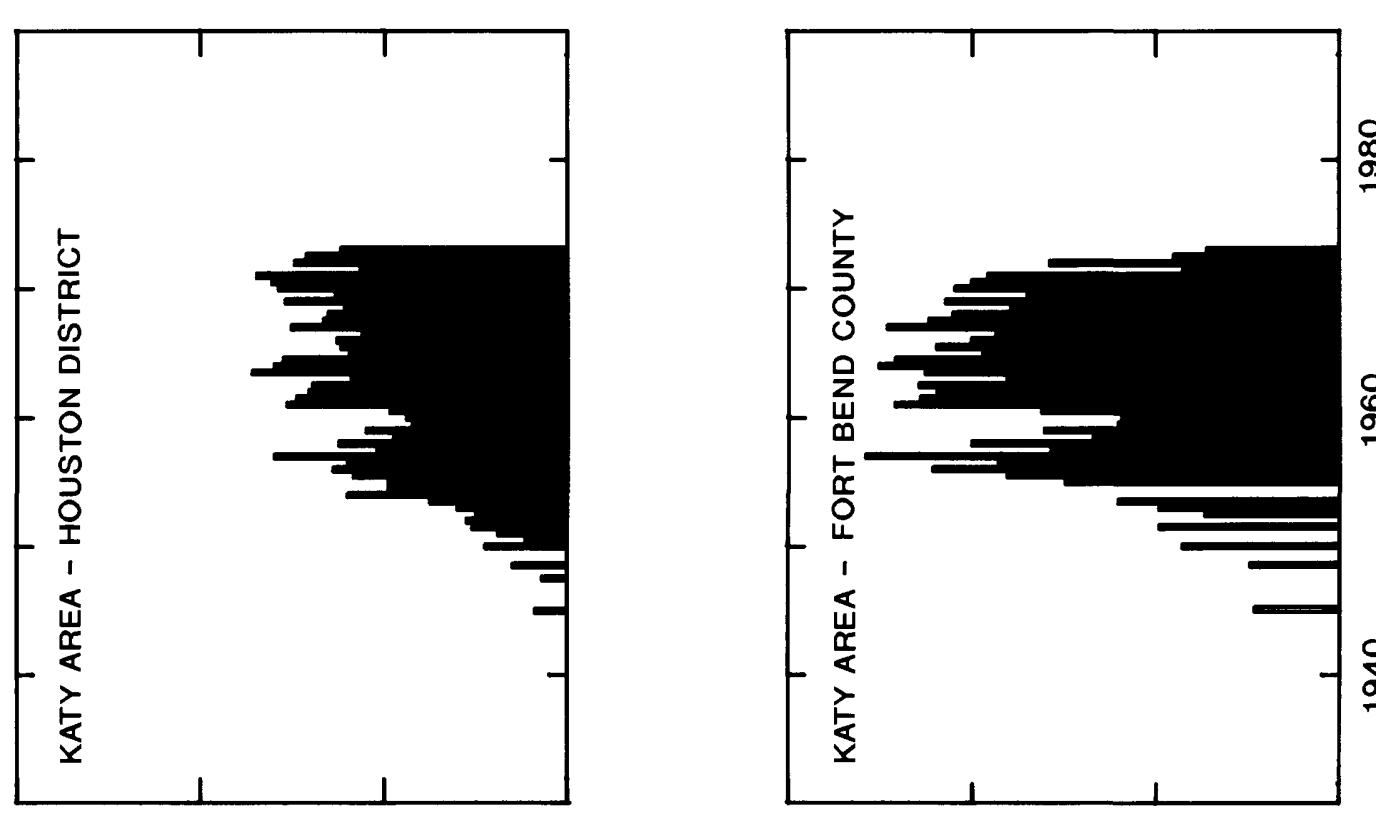

$\subseteq$

음

范

$\frac{\frac{1}{0}}{\frac{0}{0}}$

응

옹

$\stackrel{\infty}{\mp}$

ธ०

is

$\pi$

ㅇำ

$\Phi$

๙

응 응

옹 눙
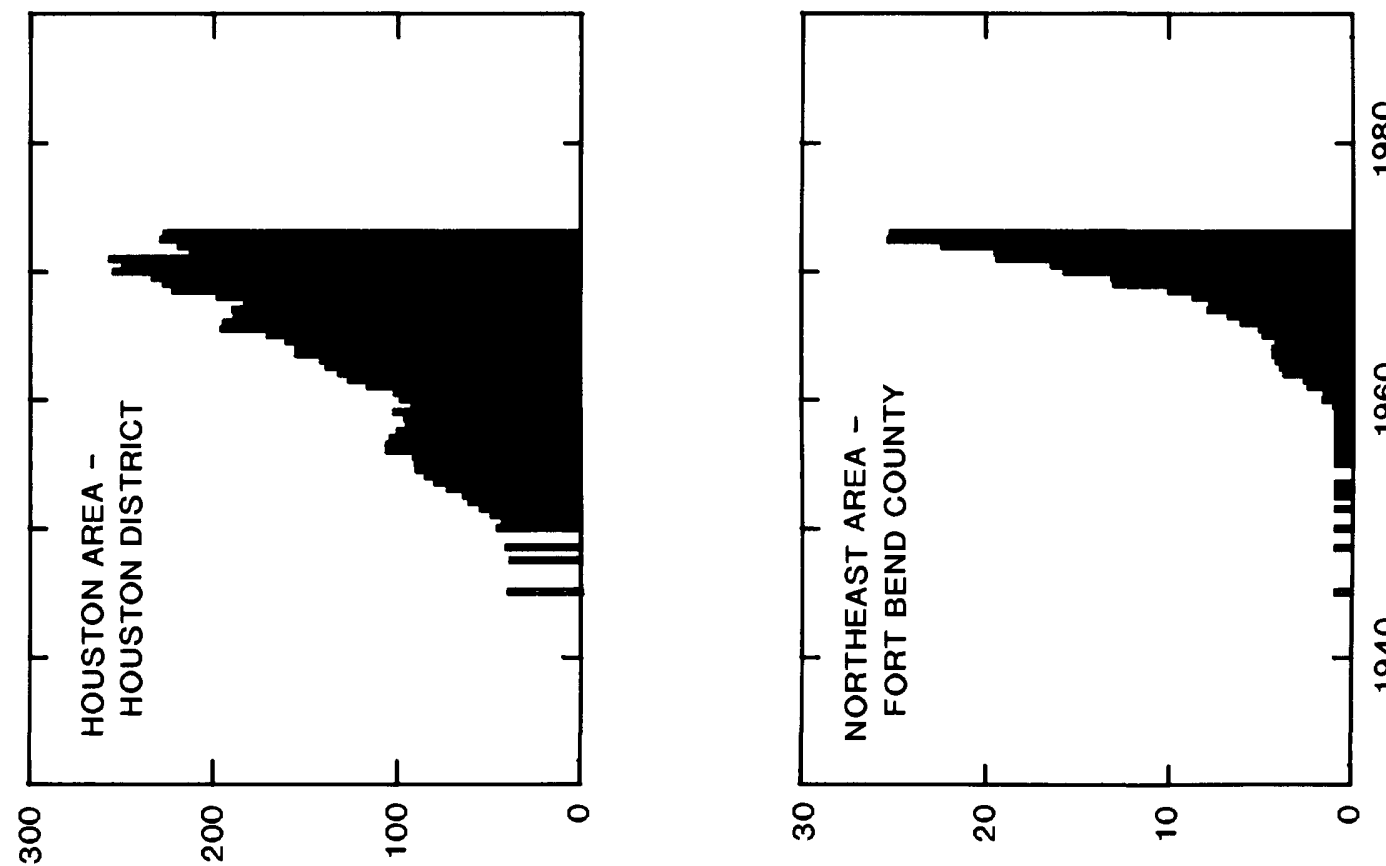

그눙

늄

क

$\frac{\infty}{\pi}$

3

苋

일

\&

可

त

$3 \stackrel{1}{1}$

은 응

잉

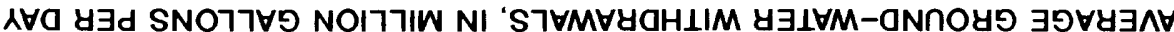


aquifers were estimated to have been about 15 to $35 \mathrm{ft}$ above land surface and nearly flat before the large-scale development of ground water began in the Houston district in about 1900 (Jorgensen, 1981, p. 421). The combined effect of the withdrawal of water from the hundreds of wells in the Houston district east of Fort Bend County has been the formation of regional cones of depression in the potentiometric surfaces of the Chicot and Evangeline aquifers. Although the depressions are centered around areas of greatest withdrawal, areas without substantial withdrawal are affected by this regional lowering of water levels. Wesselman (1972, p. 34-35), and Wood and others (1963, p. 78) attributed much of the decline in water levels in eastern Fort Bend County to withdrawals in the Houston district.

\section{Water-Level Changes in the Houston District}

Since 1969, substantial changes in ground-water withdrawals and water levels have been evident in the Houston district. Withdrawals in the eastern and southern parts of the district (Pasadena, Baytown-La Porte, NASA, and Galveston County) have declined sharply beginning in 1977 because of the increased substitution of surface water for ground water; the combined withdrawals had decreased 75 percent (132 Mgal/d) by 1986 . As a result, water levels in the eastern and southern parts of the Houston district have risen as much as $120 \mathrm{ft}$ in wells screened in the Evangeline aquifer and as much as 140 $\mathrm{ft}$ in wells screened in the Chicot aquifer between 1977 and 1985 (Williams and Ranzau, 1987, p. 18-20). Ground-water withdrawals in the Katy and Houston areas of the district, which are adjacent to and include parts of Fort Bend County, increased 35 percent (111 Mgal/d) from 1969 to 1982, then decreased 18 percent ( $75 \mathrm{Mgal} / \mathrm{d})$ from 1982 to 1986 . This withdrawal pattern is similar to that found in Fort Bend County. Water-level declines of as much as $80 \mathrm{ft}$ in wells screened in the Chicot aquifer and as much as $140 \mathrm{ft}$ in wells screened in the Evangeline aquifer have been recorded in the western part of the district (Katy and Houston areas) between 1977 and 1985 (Williams and Ranzau, 1987, p. 18-20). The lowest potentiometric surfaces of the Chicot and Evangeline aquifers, which historically have been east of downtown Houston, are now located about $8 \mathrm{mi}$ west of downtown Houston, towards Fort Bend County.

\section{Water-Level Changes in Fort Bend County}

Wesselman (1972) reported the results of water-level measurements made during 1968-69 in wells screened in the upper and lower units of the Chicot aquifer and in the Evangeline aquifer. Water levels in wells screened in these aquifers were measured during 1986-87 as part of this study to determine what changes had occurred since 1968-69. Water levels in observation wells measured between 1969 and 1987 are presented in table 4. The approximate altitudes of water levels in wells screened in the lower unit of the chicot aquifer and in the Evangeline aquifer in 1987 are shown in figures 6 and 7, 


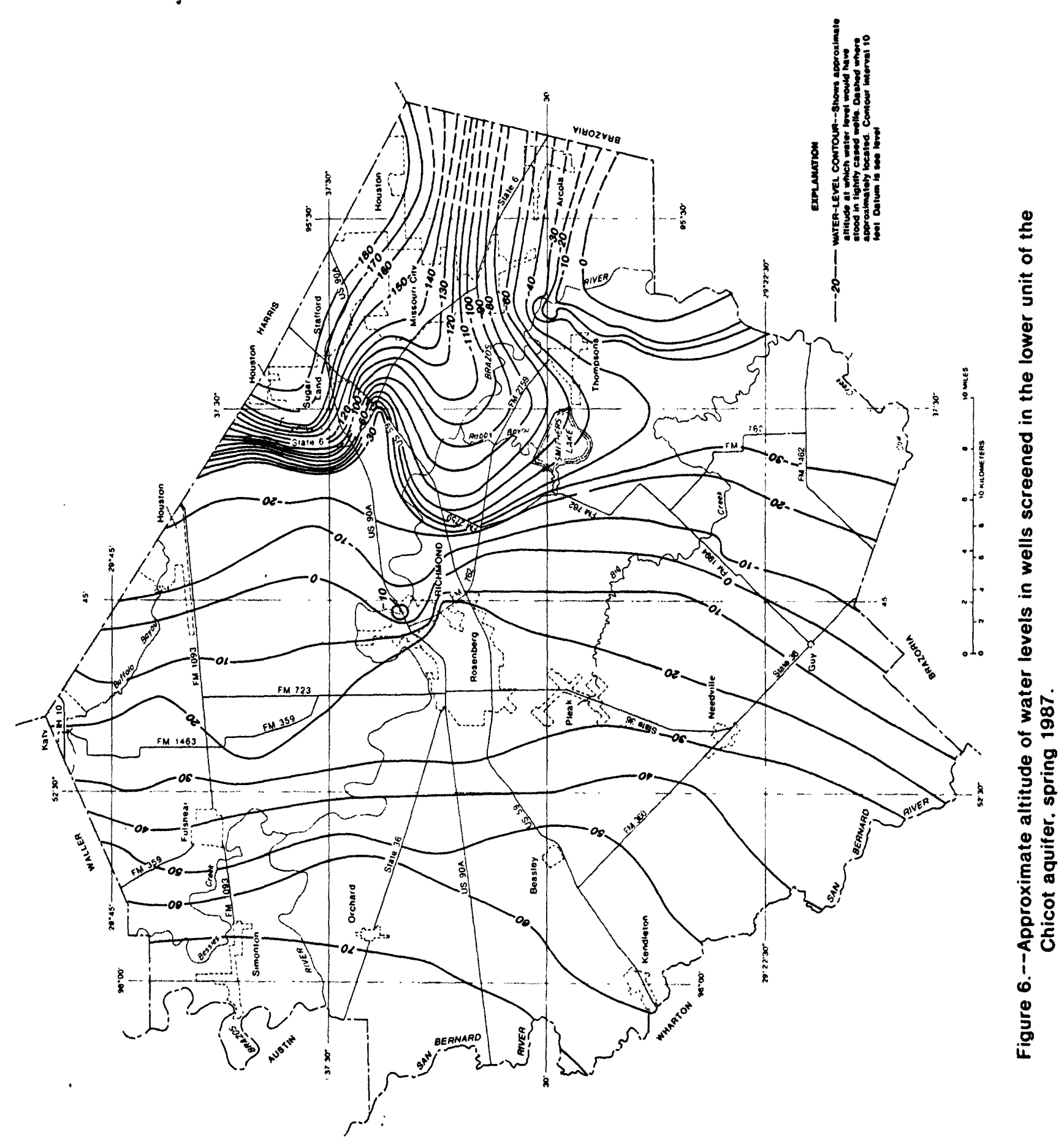

1 


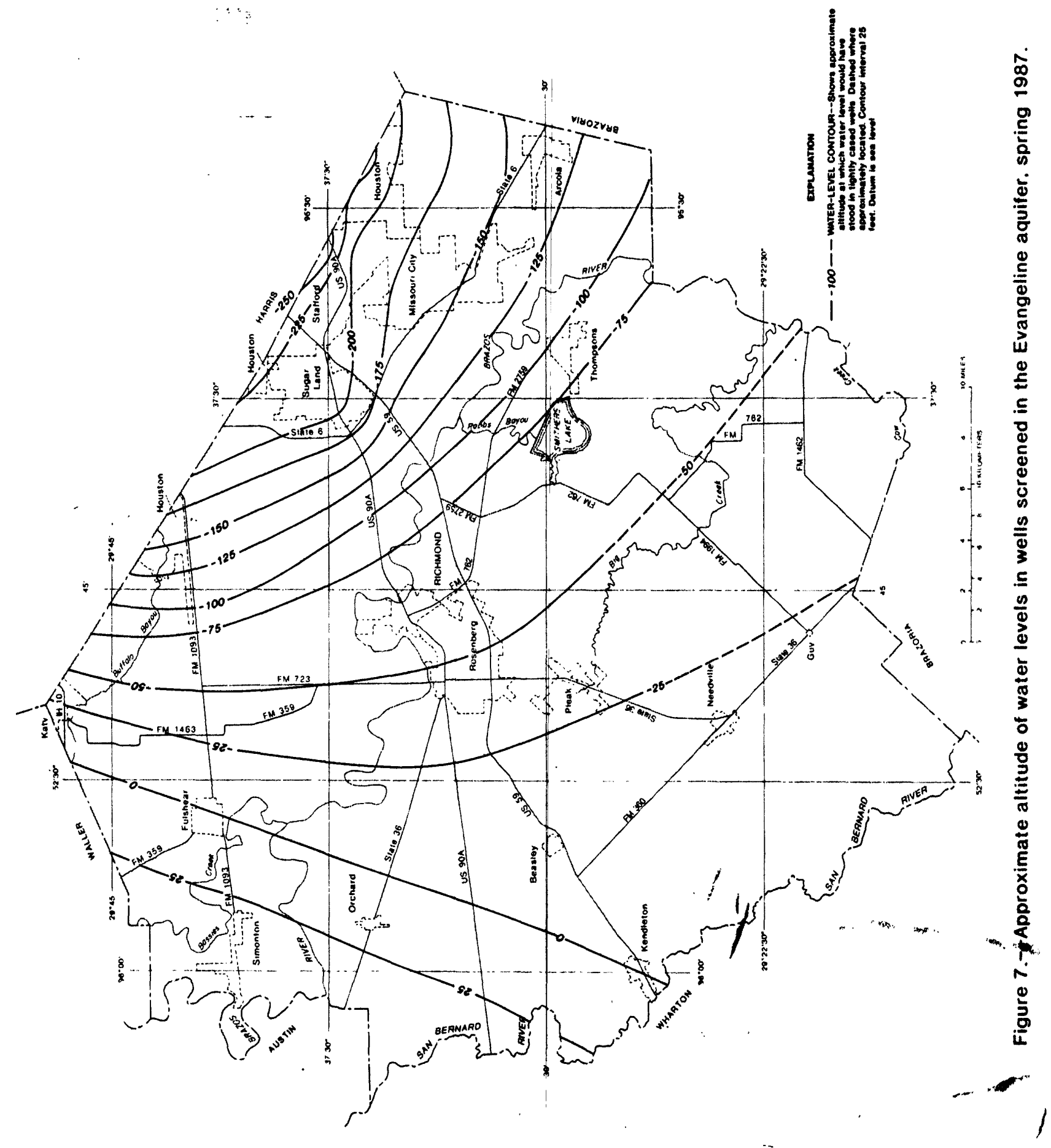


respectively. Water levels shown to be estimated on the altitude maps were based on measurements made in 1986.

\section{Upper Unit of the Chicot Aquifer}

Measurements of water levels in 81 wells were used to construct the 196869 water-level altitude map for the upper unit of the chicot aquifer (Wesselman, 1972, fig. 24). Water levels were measured in 21 of these wells and in 14 additional wells in 1986 or 1987. Comparison of the water levels indicates that there has been little change (generally rises or declines of less than $4 \mathrm{ft}$ ). In wel1 JY-65-35-101 (86 ft deep), water levels had a net rise of $0.65 \mathrm{ft}$ between 1969 and 1986 (table 4). In the adjacent well, JY-6535-102 (180 ft deep), the water level declined about $4 \mathrm{ft}$ between 1969 and 1987. Between 1947 and 1986, water levels in each well declined about $17 \mathrm{ft}$, most of which (about $13 \mathrm{ft}$ ) occurred before 1962. Wesselman (1972, p. 43) attributed much of the decline in water levels in the upper unit of the Chicot aquifer to leakage into the lower unit because there is little withdrawal from the upper unit, except in areas of irrigation.

\section{Lower Unit of the Chicot Aquifer}

Between 1947 and 1987, the estimated water-level declines in wells screened in the lower unit of the chicot aquifer ranged from less than $10 \mathrm{ft}$ along the western border of the county to more than $200 \mathrm{ft}$ in the northeastern part of the county. Between 1968-69 and 1987, water-level declines (fig. 8) ranged from less than $10 \mathrm{ft}$ in the western part of the county to about $100 \mathrm{ft}$ in the northeastern corner of the county. In the southwestern part of the county, the decline was less than $20 \mathrm{ft}$. The rate of decline in individual wells ranged from less than $0.6 \mathrm{ft} / \mathrm{yr}$ to $5 \mathrm{ft} / \mathrm{yr}$.

The localized $50-\mathrm{ft}$ rise in water levels east of Needville is the result of the closing of a sulfur-mining plant in 1984. The plant withdrew an average of $3.8 \mathrm{Mgal} / \mathrm{d}$ from 1969 to 1982 .

The Geological Survey has measured or maintained records of water-levels in 31 wells throughout the county that are screened in the lower unit of the Chicot aquifer. In 25 of the 27 wells outside of the northeast area of the county, water levels generally declined from 1969 to 1982 and have since risen a few feet or remained stable, corresponding to the overall reduction in ground-water withdrawals after 1982 (fig. 4). In the remaining two wells and in the four wells in the northeast area, water levels have continuously declined after 1969. Local ground-water withdrawals in the northeastern part have generally been increasing since 1969 . 


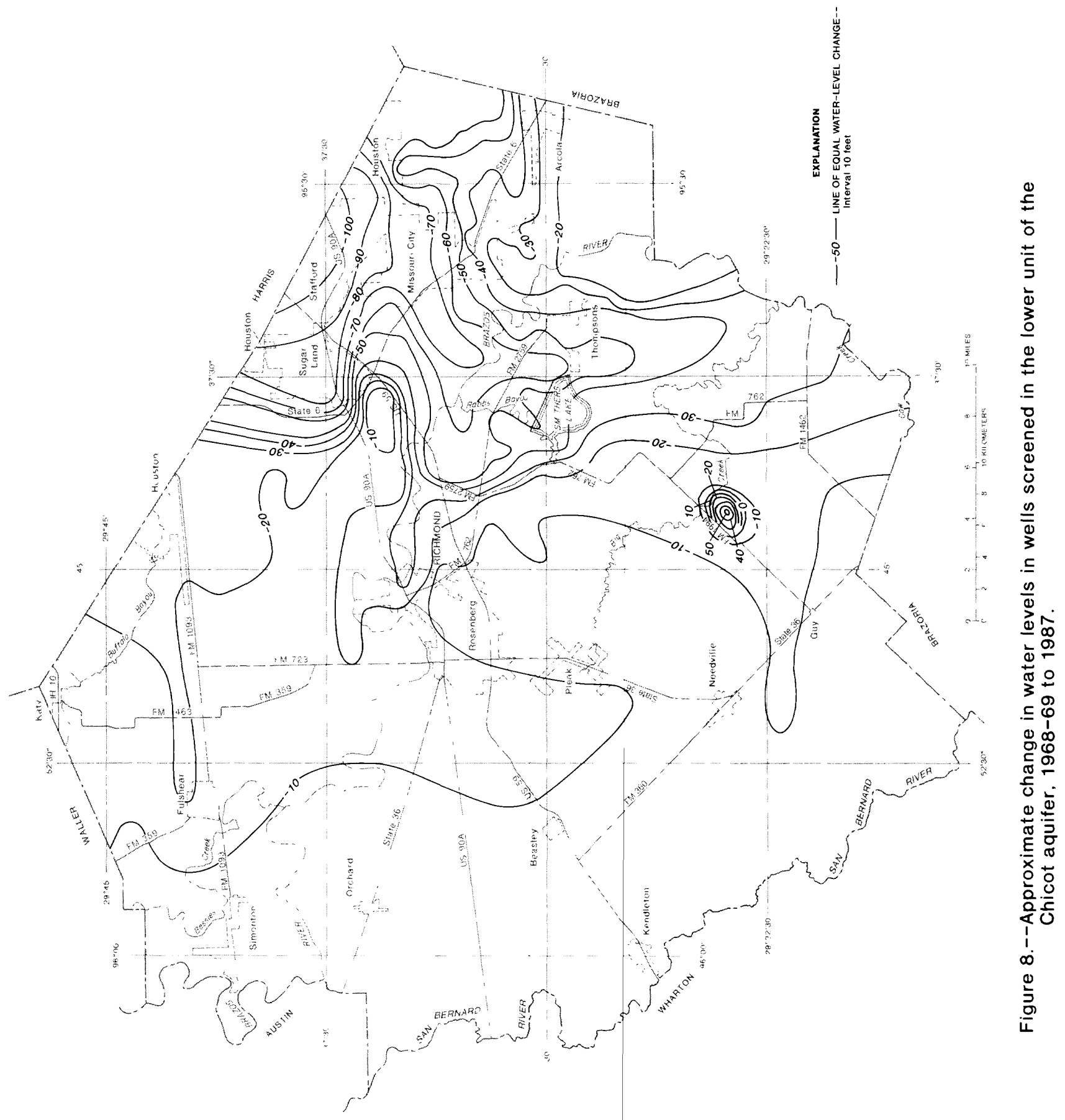


The changes in water levels that typify most of the observation wells in the county are shown in figure 9 . The overall rate of decline in both wells from 1969 to 1987 is about $0.5 \mathrm{ft} / \mathrm{yr}$, al though between 1969 and 1983, the rate of decline was about $1.0 \mathrm{ft} / \mathrm{yr}$. Between 1983 and 1987, water levels have risen $4 \mathrm{ft}$ in the Katy area irrigation well JY-65-17-201 and $6 \mathrm{ft}$ in the irrigation well JY-65-33-504, located in the southwestern part of the county.

Water levels in selected wells located in the northeastern part of the county are illustrated in figure 10. Water levels in well JY-65-29-701 declined at a rate of $1.8 \mathrm{ft} / \mathrm{yr}$ from 1969 to 1986 . Water levels in well JY65-27-303 declined at a rate of $4.7 \mathrm{ft} / \mathrm{yr}$ from 1969 to 1986.

\section{Evangel ine Aquifer}

Between 1947 and 1987, estimated water-level declines ranged from less than $75 \mathrm{ft}$ in the southwestern part to $225 \mathrm{ft}$ in the northeastern part of the county. In much of the northeastern one-quarter of the county the declines were more than $100 \mathrm{ft}$. Between 1968-69 and 1987, water-level declines in wells in the Evangeline aquifer (fig. 11) ranged from less than $25 \mathrm{ft}$ in the northwestern part to $125 \mathrm{ft}$ in the northeastern part of the county. The rate of decline in individual wells ranged from less than $1.5 \mathrm{ft} / \mathrm{yr}$ to $7.0 \mathrm{ft} / \mathrm{yr}$. In the southwestern one-quarter of the county, the decline in water levels was less than $50 \mathrm{ft}$.

The Geological Survey has measured water levels in nine wells that were completed in the Evangeline aquifer. The wells are located along the Fort Bend-Harris County line from Katy to Missouri City. Water levels of six of the wells are shown in figure 12. Water-level records from only three of the wells span most of the years 1969 to 1987 (fig. 12).

Near Katy, water levels declined $1.0 \mathrm{ft} / \mathrm{yr}$ in well JY-65-17-404 between 1970-87, and 0.6 ft/yr in well JY-65-10-812 between 1983-87. In the northeastern part of the county, water levels declined $5.6 \mathrm{ft} / \mathrm{yr}$ in well JY-65-27302 between 1969-86 and $8.2 \mathrm{ft} / \mathrm{yr}$ in well JY-65-28-311 between 1974-87. The less than 5 feet of water-level decline in wells JY-65-19-509, JY-65-28-309, and JY-65-28-311 (shown in figure 12), and in wells JY-65-28-313 and JY-65-28508 (not shown in figure 12) between 1985-87 may indicate a recent stabilization of the water levels in the northeastern part of the county.

\section{LAND-SURFACE SUBSIDENCE}

Land-surface subsidence has been studied extensively in the Houston area. As part of these studies, subsidence maps were prepared that included Fort Bend County (Gabrysch and Bonnet, 1975, figs. 8-10, and Gabrysch, 1982, figs. 14-16). These maps were based on differences in altitudes of bench marks 


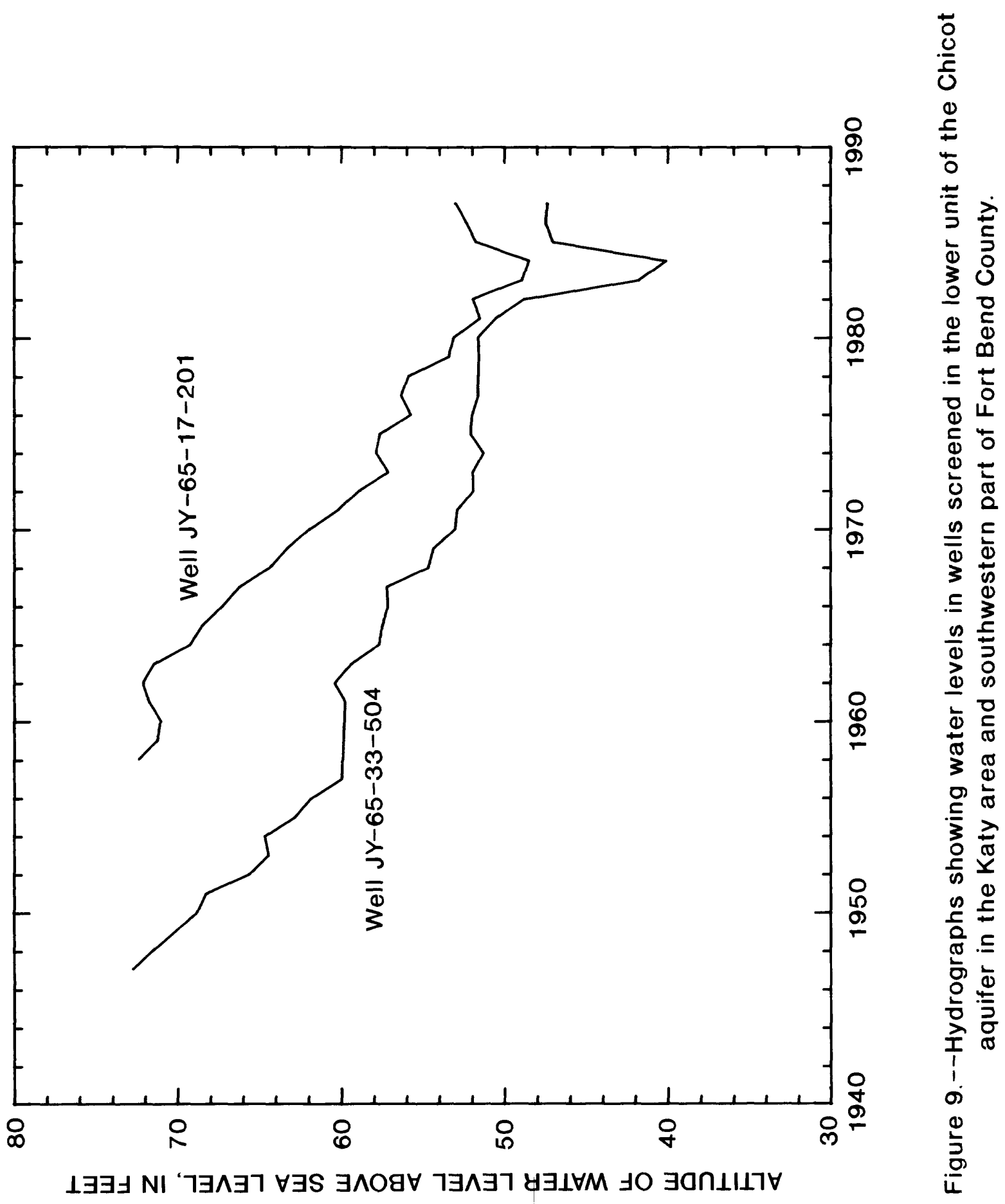




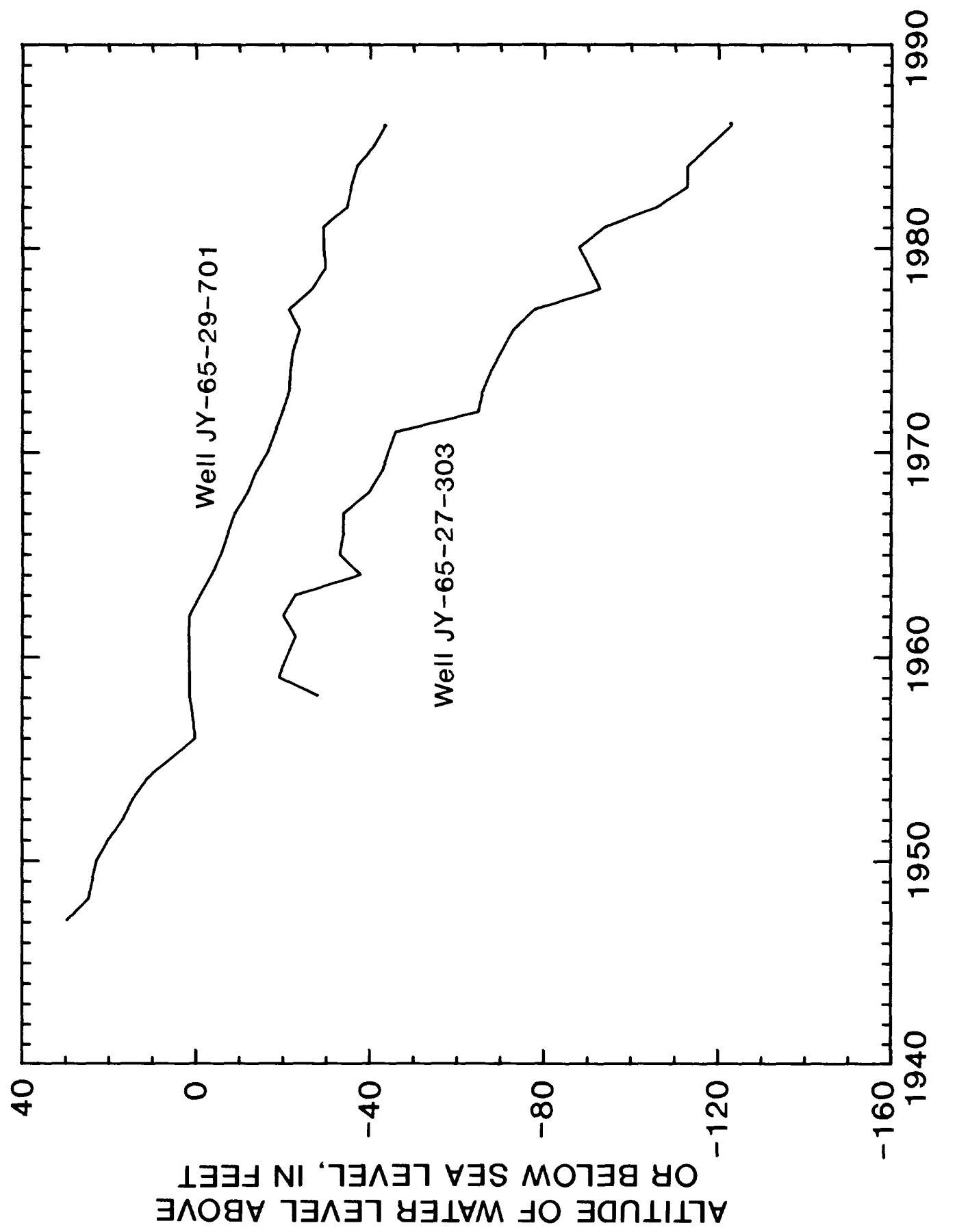

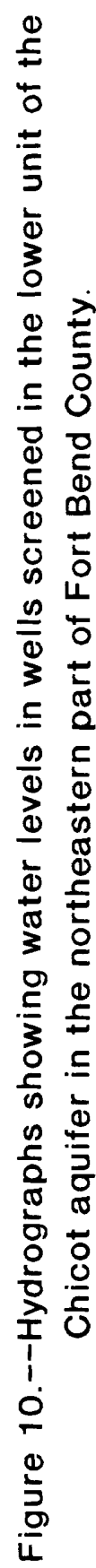




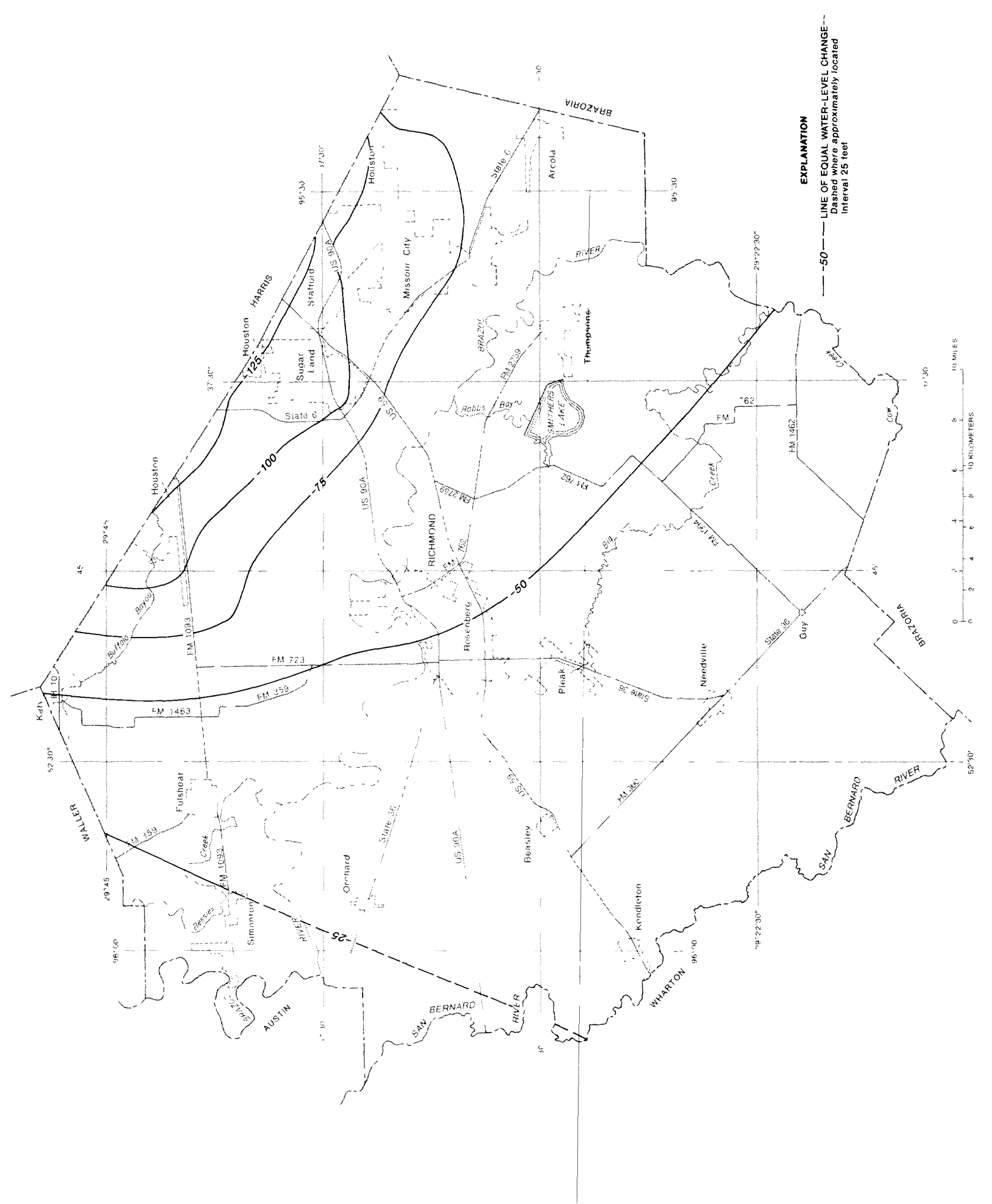

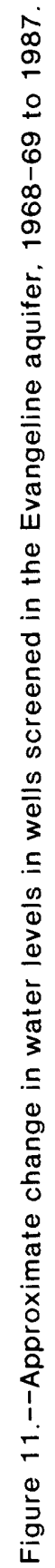




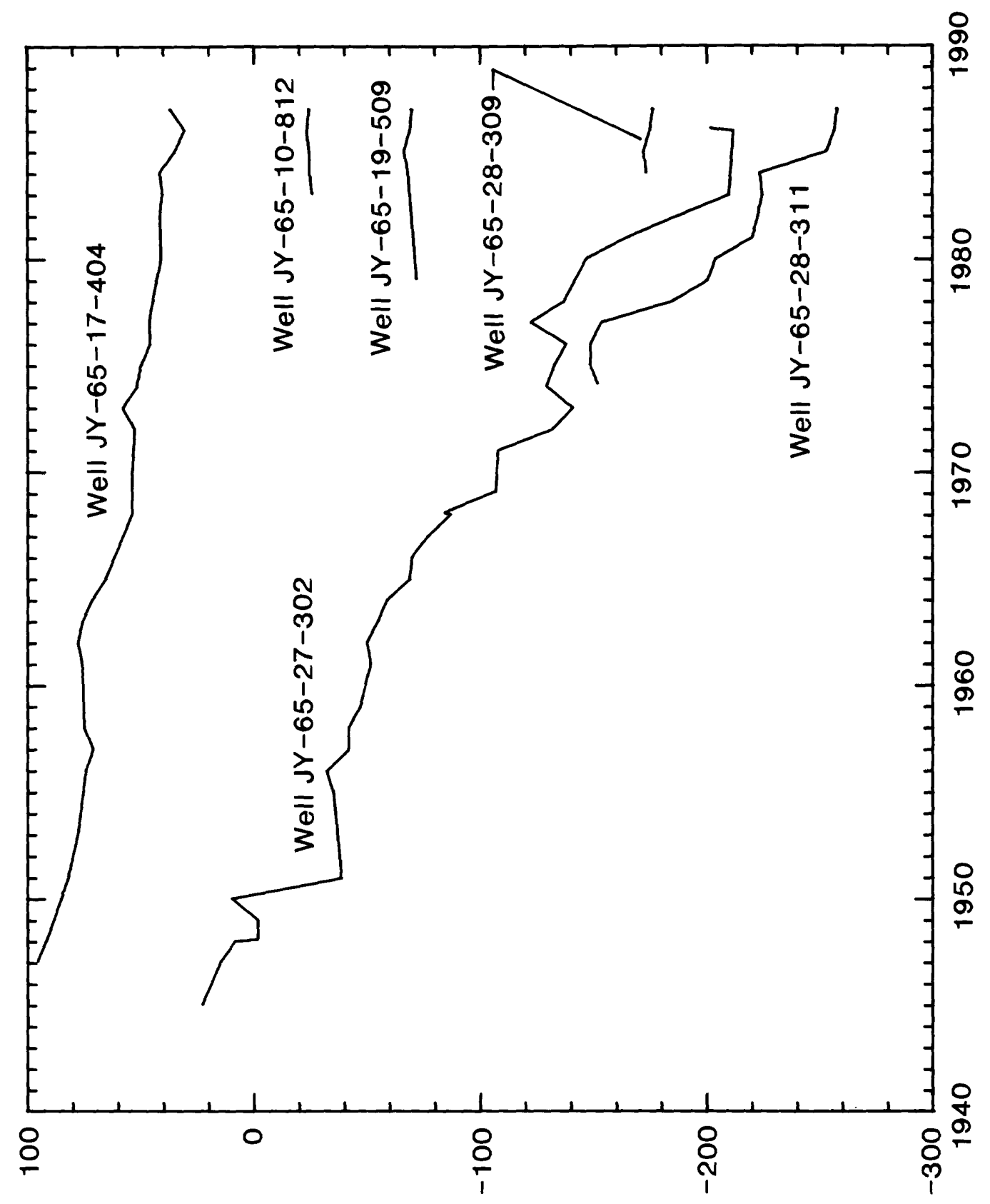

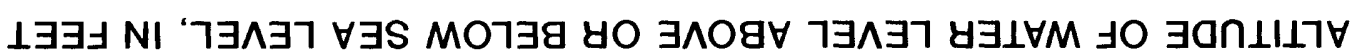

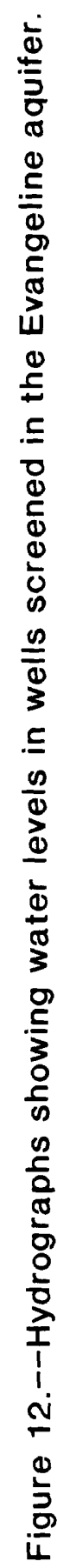


measured by the National Geodetic Survey. Altitudes of bench marks in most of Fort Bend County were determined in 1942-43; altitudes in the eastern part of Fort Bend County were redetermined in 1973 and 1978. Subsidence for the periods between these surveys was computed for the eastern part of the county. Subsidence in Fort Bend County between 1906 and 1943 has been estimated from subsidence measured in Harris and Galveston Counties. Between 1906 and 1978, the land surface in about 65 percent of Fort Bend County $\left(580 \mathrm{mi}^{2}\right)$ subsided more than $0.5 \mathrm{ft}$; about 5 percent of the county $\left(40 \mathrm{mi}^{2}\right)$ subsided more than 2 $\mathrm{ft}$. The maximum measured subsidence between 1943 and 1978 was $2.264 \mathrm{ft}$ at bench mark K805, located $1 \mathrm{mi}$ northwest of Missouri City. From 1973 to 1978, the measured subsidence ranged from $0.014 \mathrm{ft}$ at bench mark V1212, $6 \mathrm{mi}$ south of Katy, to $0.486 \mathrm{ft}$ at bench mark K805.

Land-surface subsidence is caused by the compaction of clay sediments. Gabrysch (1969, p. 44) stated: "Withdrawal of water from the artesian aquifers results in an immediate decrease in hydraulic pressure, which partially supports the weight of the overburden. With reduction in pressure, an additional load is transferred to the skeleton of the aquifers, and a pressure difference between the sands and clays causes water to move from the clays to the sands. Most of this process of sediment compaction takes place in the clays." Land-surface subsidence is directly dependent on the thickness of clay beds within the aquifers and the decline in water pressure in the sands adjacent to the clay beds.

\section{Thickness of Clay}

Gabrysch (1969, fig. 11) related the thickness of clay, in percent, to subsidence in the Houston district. Among the factors affecting this relation are the clay mineralogy, the thickness of individual clay beds, and the compressibility of the clays. Of the clay minerals, montmorillonite is the most compressible. The most common clay assemblage in the Houston district is montmorillonite, illite, and kaolinite (Gabrysch, 1969, p. 42). The time lag between an increase in stress (water-level decline) and the resulting compaction of a clay bed is directly related to thickness of the individual bed. In general, the thickness of individual clay beds increases with depth in the Houston district.

The compressibility of clays is thought to be inversely related to its age and depth of burial (Gabrysch, 1982, p. 56). Analysis of data from compaction monitors installed at various depths in southern Harris County indicated that a large part (55 percent) of the subsidence in this area resulted from compaction of clays in the younger and shallower Chicot aquifer (Gabrysch and Bonnet, 1975, p. 15). The depth to which compaction occurs is unknown. It is assumed (Gabrysch, 1982, p. 56) that because of the withdrawal of water, all the compaction occurs above the base of the Evangeline aquifer. 
In northern Harris County, compaction of clays within the Evangeline is thought to be more important, caused, in part, by the decreased amount of clay in the Chicot aquifer (Jorgensen, 1981, p. 418).

For this study, the approximate clay thicknesses in the Chicot aquifer (upper and lower units combined) and Evangeline aquifer were determined from electric logs. The clay thicknesses are for the total thickness of each aquifer. The distribution of the locations of the electric logs is biased towards the eight salt domes in the county, where oil and gas exploration has occurred. The thickness of clay in the chicot aquifer increases from less than $150 \mathrm{ft}$ in the northwestern part of the county to more than $350 \mathrm{ft}$ in the eastern part of the county (fig. 13). The increase is caused by increased thickness of the aquifer along the regional dip towards the coast and a corresponding increase in the percentage of clay. The clay increases from an average of about 25 percent of the aquifer in the northwestern part of the county to an average of about 45 percent in the eastern part.

The thickness of clay in the Evangeline aquifer (fig. 14) increases from about $700 \mathrm{ft}$ in the northwestern part of the county to about $1,100 \mathrm{ft}$ in the eastern part. The thickness of the aquifer also increases in the same direction from about $1,300 \mathrm{ft}$ to more than $2,000 \mathrm{ft}$, but the percentage of clay remains relatively constant at about 55 percent.

The total thickness of clay in both the Chicot and Evangeline aquifers (fig. 15) increases from an average of about $850 \mathrm{ft}$ in the northwestern part of the county to an average of about $1,500 \mathrm{ft}$ in the eastern part. The percentage of total clay thickness varies slightly, with a mean of about 50 percent.

\section{Relation Between Thickness of Clay, Water-Level Declines, and Subsidence}

The relative susceptibility of areas with different amounts of clay and rates of water-level decline to clay compaction can be made by comparison of specific-unit compaction. Specific-unit compaction is defined as the compaction of deposits per unit of clay thickness per unit of increase in applied stress during a specified time.

Gabrysch (1982, table 1) calculated smaller values of specific-unit compaction for 1906-43 than for 1943-64, 1964-73, and 1973-78. His explanation was that the aquifers had been subjected to a natural stress in geologic time (preconsolidation stress) before withdrawal of ground water had begun. He further speculated that the higher (by almost one order of magnitude) specific-unit compaction values determined for the later leveling periods were caused by preconsolidation stress that had subsequently been exceeded, and that greater amounts of subsidence per unit of water-level 


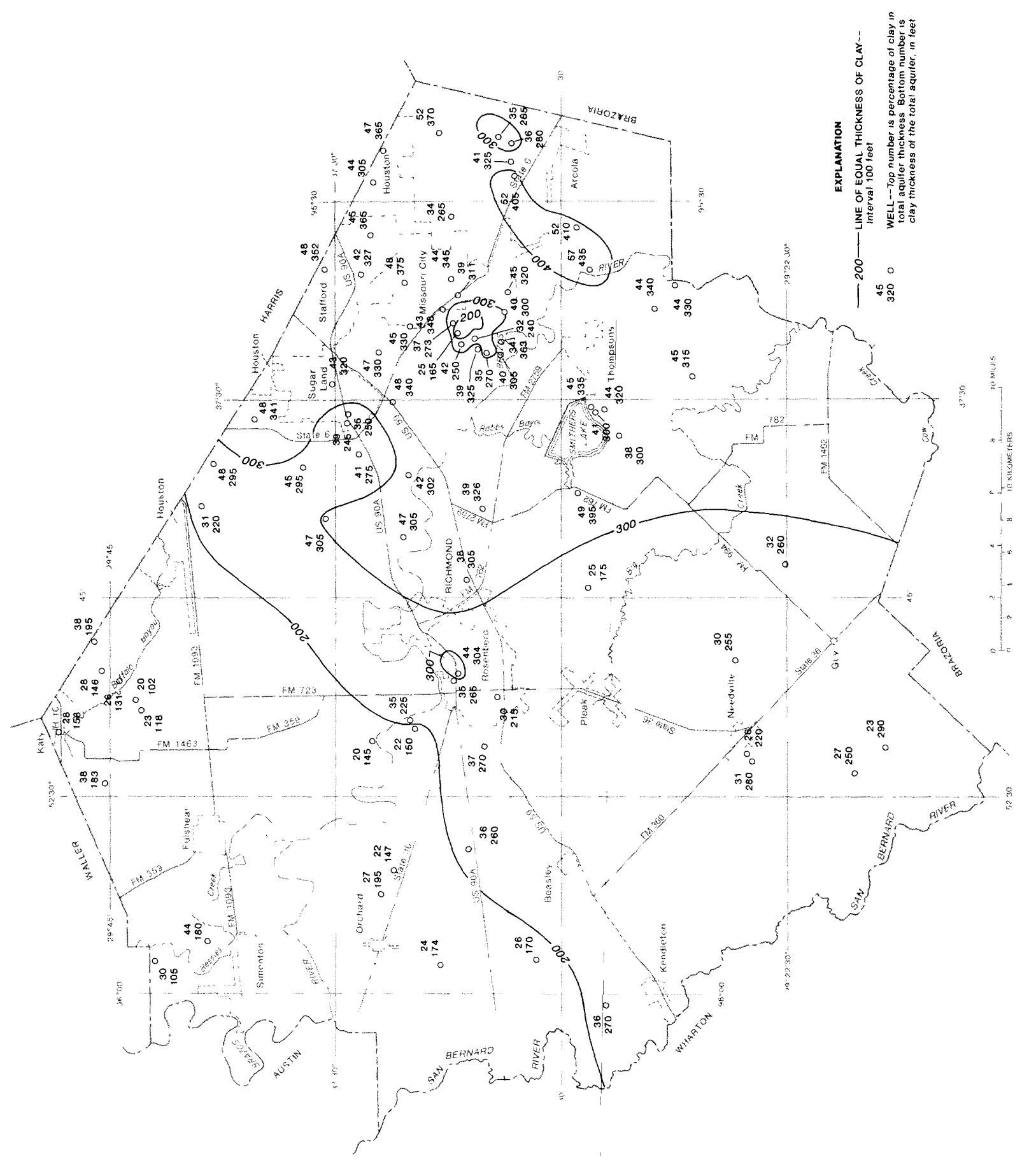

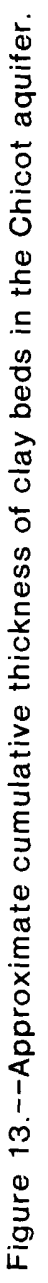




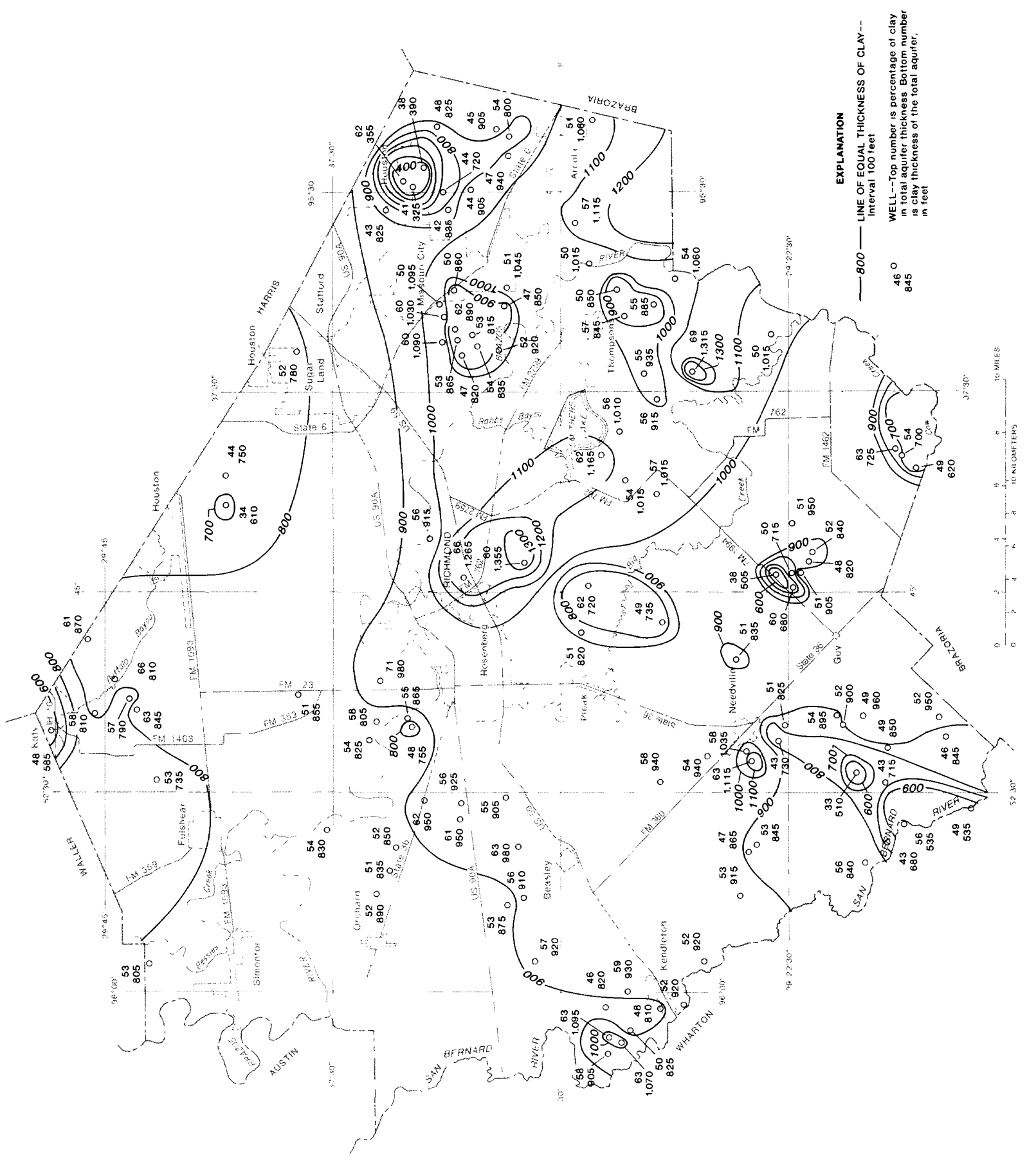

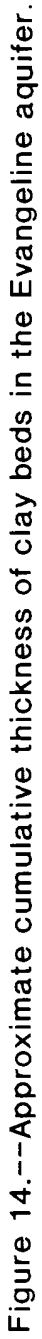




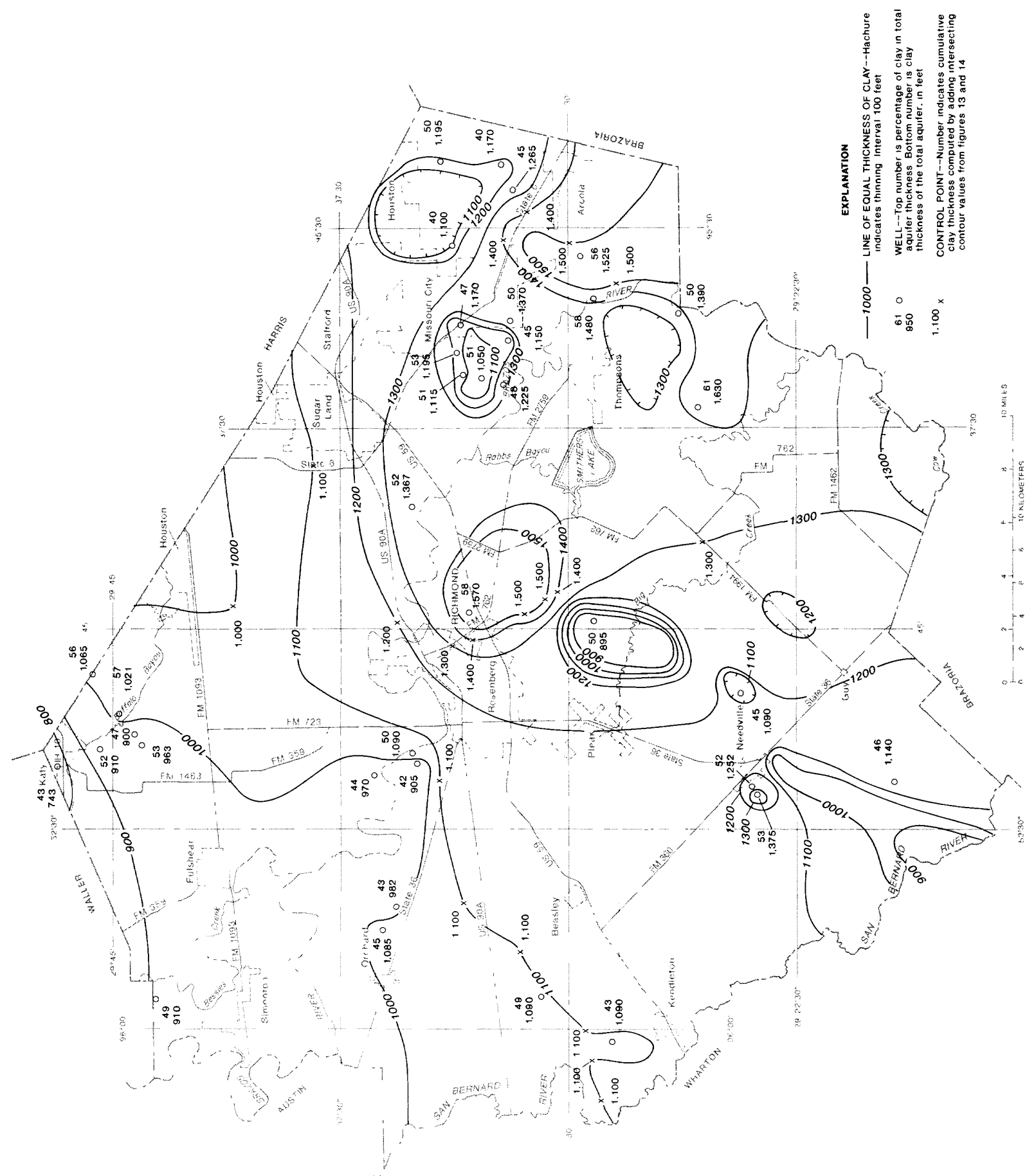

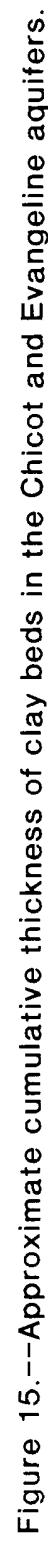


decline could be expected in the future. Most clay compaction that occurs after the preconsolidation stress is exceeded is permanent. The specific-unit compaction values for 1906-78 indicated that the younger clays nearer the coast were more susceptible to compaction than the older, more inland clays (Gabrysch, 1982, p. 65).

Locations of the compaction monitor sites and calculated values of specific-unit compaction are shown in figure 16. Much of Fort Bend County is as equidistant from the coast as the Addicks, Houston-Northeast, HoustonSouthwest, and Lake Houston compaction monitor sites, and the geologic setting is equivalent. The values of specific-unit compaction for Fort Bend County were calculated using measured subsidence, estimated water-level declines, and estimated total thickness of $\mathrm{clay}$ in the Chicot and the Evangeline aquifers (fig. 15). An average water-level decline was determined by dividing the estimated water-level decline by 1.33 , the ratio of maximum to average waterlevel decline reported by Gabrysch (1982, p. 64, table 1). The values were averaged for several bench marks in a particular area. Specific-unit compaction values calculated for the Katy area using the average water-level decline are presented in table 5 (Supplemental Data).

For 1943-73, the specific-unit compaction values along the Fort BendHarris County line were similar, averaging $1.2 \times 10^{-5} \mathrm{ft}^{-1}$, which indicates a similarity in the compressibility of the clays along this border. These values also are similar to the value at the inland Addicks compaction monitor site $\left(1.2 \times 10^{-5} \mathrm{ft}^{-1}\right.$ shown in figure 16$)$. In contrast, the specific-unit compaction values for $1943-73$ averaged about $5.1 \times 10^{-5} \mathrm{ft}^{-1}$ at the sites nearer the coast (Baytown, Clear Lake, Moses Lake, Pasadena, and Seabrook). For 1906-78, the specific-unit compaction value at Katy $\left(1.2 \times 10^{-5} \mathrm{ft}^{-1}\right.$ shown in table 5) is comparable to the average value $\left(1.2 \times 10^{-5} \mathrm{ft}^{-1}\right)$ of the inland sites at Addicks, Houston-Northeast, Houston-Southwest, and Lake Houston. The specific-unit compaction values of the five monitor sites nearer the coast averaged $3.1 \times 10^{-5} \mathrm{ft}^{-1}$ during 1906-78. Comparison of the specific-unit compaction values along the northeast boundary of Fort Bend County with the more inland compaction monitor sites of the Houston district indicates a similarity in their compressibility, which is less than that of the sites nearer the coast.

In the central part of Fort Bend County, the specific-unit compaction for 1943-73 averaged about $6.7 \times 10^{-6} \mathrm{ft}^{-1}$. The smaller values may indicate that the stress caused by water-level declines was less than the preconsolidation stress of the clays during all or most of the period. The compaction of the clays is less for stresses (water-level declines) smaller than the preconsolidation stress. The two benchmarks with relatively large specific-unit compaction $\left(1.5 \times 10^{-3} \mathrm{ft}^{-1}\right)$ are located over the Thompson 011 and gas field. 


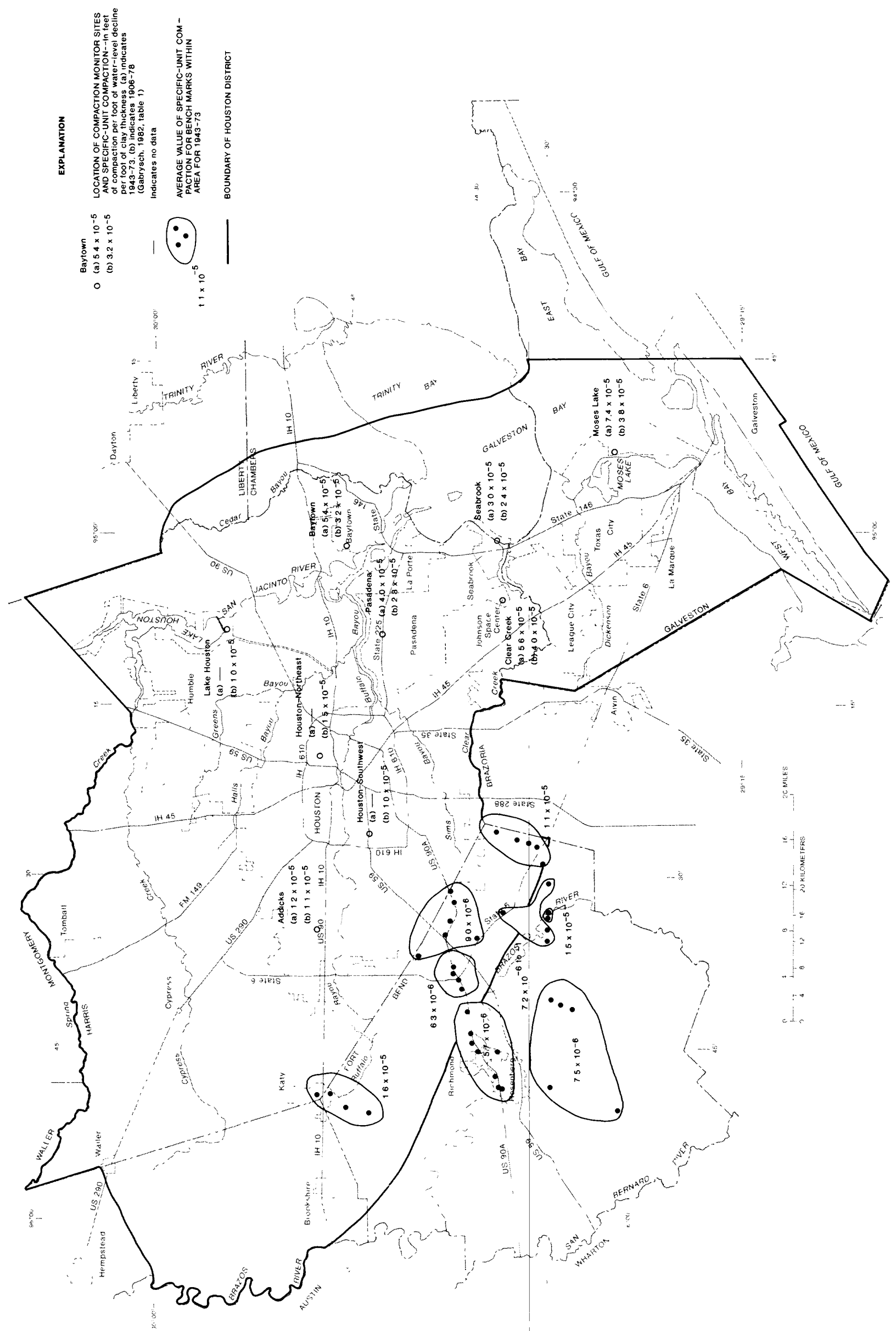

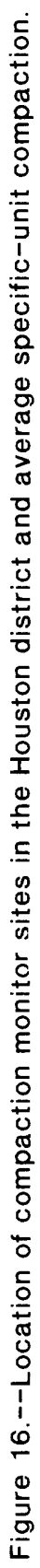


Holzer (1981, table 1) estimated that for stresses caused by water-level declines less than the preconsolidation stress, the ratio of land subsidence to water-level declines in the Chicot aquifer ranged from 0.007 to 0.011 . The declines were conservatively estimated to be equivalent to depth to water from land surface in wells because the original potentiometric surface of the aquifers was above land surface. For water-level declines greater than the preconsolidation stress, the ratio ranged from 0.027 to 0.036 . The relation between the subsidence of bench mark $Y 7$ at Katy and the depth to water is shown in figure 17 . When the depth to water exceeded about $75 \mathrm{ft}$, the ratio of subsidence to water-level decline increased from about 0.004 to 0.010 , an indication from Holzer's work that the preconsolidation stress had been exceeded. Corresponding to this ratio increase, the specific-unit compaction increased from $4.3 \times 10^{-6} \mathrm{ft}^{-1}$ for $1906-54$ to $2.7 \times 10^{-5} \mathrm{ft}^{-1}$ for 1954-78.

Holzer (1981) estimated that the preconsolidation stress was equivalent to about 100 to $200 \mathrm{ft}$ of water-level decline (from land surface) in the Chicot aquifer. However, Meyer and Carr (1979, p. 13) estimated that the preconsolidation stress was equivalent to about $70 \mathrm{ft}$ of decline in the Houston district. At Katy, the preconsolidation stress apparently is equivalent to about $75 \mathrm{ft}$ of decline (fig. 17).

In 1943, the depth to water in wells in the lower unit of the Chicot aquifer ranged from about $25 \mathrm{ft}$ to $50 \mathrm{ft}$ below land surface. Only in the Katy area and in the far northeastern corner of the county were water levels in wells between 50 and $65 \mathrm{ft}$ below 1 and surface. Gabrysch and Bonnet (1975, fig. 10) estimated that the only subsidence in Fort Bend County between 190643 (less than $0.4 \mathrm{ft}$ ) occurred in the Katy area and northeastern part.

By 1973, the depth to water in wells completed in the lower unit of the Chicot aquifer ranged from about $50 \mathrm{ft}$ in the southern and western parts of the county to $200 \mathrm{ft}$ in the northeastern corner of the county. In the northeastern part of the county, the depth to water in wells was greater than 70 $\mathrm{ft}$. The measured subsidence between 1943 and 1973 ranged from $0.5 \mathrm{ft}$ to almost $2.0 \mathrm{ft}$ (Gabrysch and Bonnet, 1975, fig. 9). The decline in water levels and the amount of subsidence indicate that the preconsolidation stress of the clays had been exceeded. Holzer's data indicate that for an additional $100 \mathrm{ft}$ of water-level decline above the preconsolidation stress, about $3 \mathrm{ft}$ of subsidence will occur. Less subsidence for an equivalent water-level decline (about $1 \mathrm{ft}$ of subsidence per $100 \mathrm{ft}$ of decline) is expected in the western and southwestern parts of the county where the preconsolidation stress probably has not been exceeded. In 1987, the depth to water in wells in the lower unit of the Chicot aquifer was still less than $70 \mathrm{ft}$ in the western and southwestern parts of the county. 


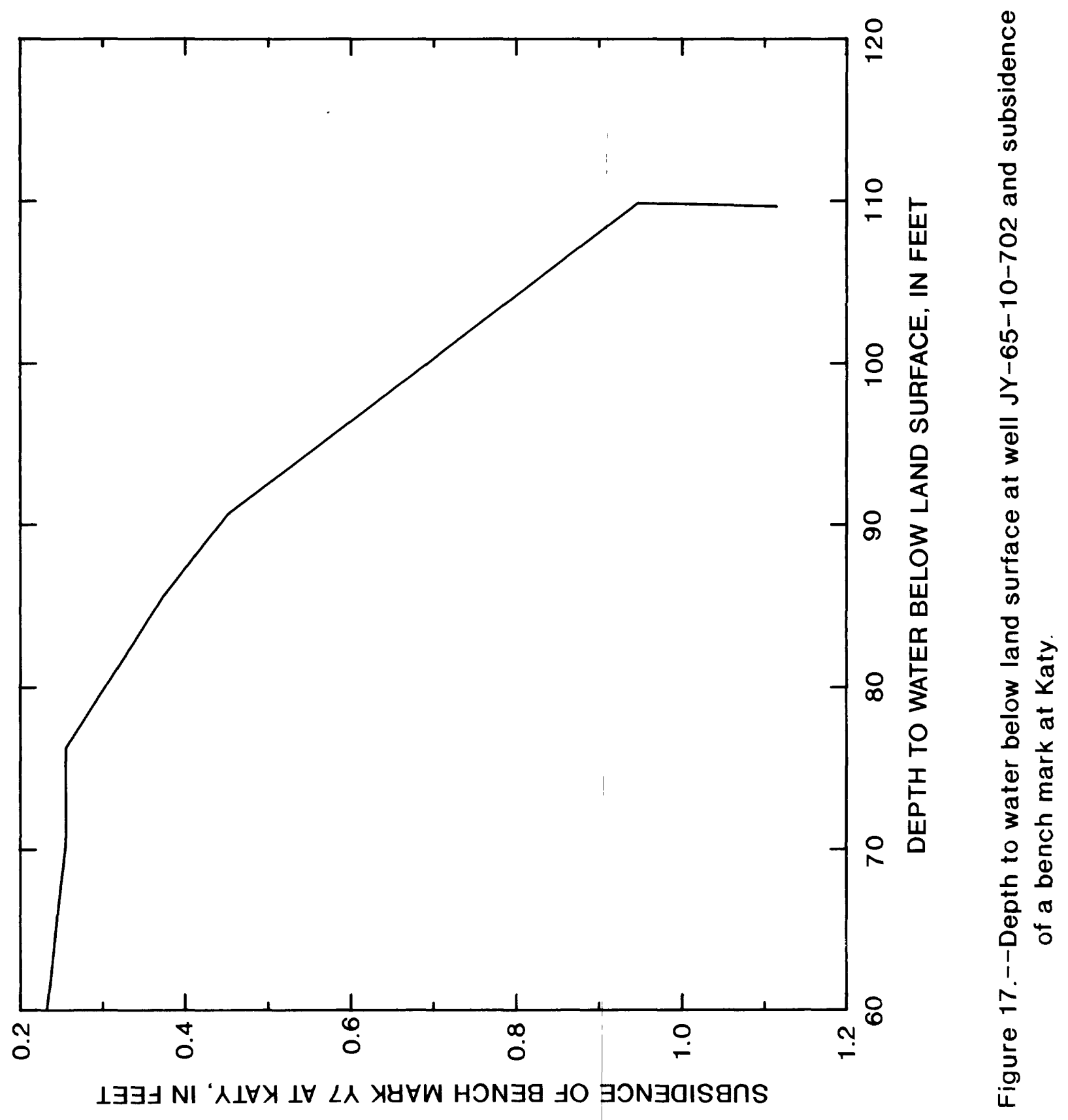




\section{Areas Most Susceptible to Land-Surface Subsidence}

The susceptibility of areas in Fort Bend County to 1 and-surface subsidence is dependent on the total thickness and compressibility of the clays and on the water-level declines (stress) to which the clays have been subjected. The total thickness and compressibility of the clays, and, therefore, the susceptibility to subsidence, increase towards the coast. The greatest thickness of $\mathrm{clay}$ and most compressible clays are in the eastern half of the county adjacent to Brazoria County. In the northeastern one-quarter of Fort Bend County, stress related to water-level decline probably has exceeded the preconsolidation stress of the clays. Consequently, the amount of subsidence per unit of water-level decline in this area is expected to be three times greater than in the southwestern one-quarter of the county. The northeastern part is the most susceptible to subsidence because it is in the area where the largest water-level declines have occurred and where some of the thickest amounts of the most compressible clay in the county are located.

\section{QUALITY OF GROUND WATER}

Wesselman (1972, fig. 7) reported that fresh ground water is present throughout Fort Bend County, usually above the base of the Evengeline aquifer. This water is suitable for public supply, irrigation, and industrial uses. A11 public water systems in Fort Bend County meet the State's drinking-water standards for heavy metals, organic compounds, and radiological properties according to the Texas Department of Health (1983). Within the freshwater zone, water containing dissolved-solids concentrations greater than $1,000 \mathrm{mg} / \mathrm{L}$ is present in the vicinity of six shallow salt domes (Wesselman, 1972, fig. 7).

Water samples from 30 wells were analyzed in 1986 for calcium, magnesium, sodium, potassium, bicarbonate, sulfate, and chloride. Water samples from about 90 wells were analyzed for specific conductance, $\mathrm{pH}$, temperature, bicarbonate, and chloride in 1986 and 1987. Water samples from an additional 21 wells were analyzed by the Geological Survey between 1969 and 1986 . Water from an additional 40 wells was analyzed by private laboratories since 1969. The Texas Department of Health provided analyses of the water from 61 wells. The results of the analyses are presented in table 6 (Supplemental Data).

Fifty-one wells located throughout the county, including areas adjacent to the shallow salt domes and areas of large water-level decline, had been sampled at least twice between 1969 and 1987. The comparison of these analyses, or the analyses between nearby wells similarly completed but sampled years apart, indicate that there has been no substantial change in the concentrations of dissolved constituents since 1969. 
Bicarbonate is the predominant anion in all the water samples. In the vicinity of the shallow salt domes, the second most common anion is chloride, or the percentages of chloride and bicarbonate ions are equal. Sodium is the predominant cation in most water samples from the Evangeline aquifer; the most common cations in water from both units of the Chicot aquifer are calcium and sodium. Water samples from the Evangeline aquifer can be characterized as a sodium bicarbonate type, whereas water samples from both units of the Chicot aquifer are a calcium or sodium bicarbonate type, or a calcium-sodium bicarbonate type.

A statistical summary of the concentrations of major dissolved ions and the values of specific conductance, hardness, and sodium adsorption ratio in water samples from each aquifer from this study and from Wesselman (1972, table 7) is presented in table 7 (Supplemental Data). For a well with multiple chemical analyses, the median values of the individual analyses were used to determine the median values for samples from the aquifer. About 20 analyses with high chloride concentrations (all from wells near a salt dome) were not used, because water from these wells generally is not used.

The median concentrations of calcium, magnesium, bicarbonate, and chloride decreased in water samples taken from the progressively deeper aquifers (the upper unit of the Chicot to the Evangeline aquifer). The median concentrations of sodium plus potassium, and sulfate were relatively constant in the aquifers. The median hardness of water samples decreased with depth from a very hard $258 \mathrm{mg} / \mathrm{L}$ in the upper unit of the Chicot aquifer, to $182 \mathrm{mg} / \mathrm{L}$ (hard) in the lower unit of the Chicot aquifer, to $105 \mathrm{mg} / \mathrm{L}$ (moderately hard) in the Evangeline aquifer. Also, the median concentration of dissolved solids decreased from $475 \mathrm{mg} / \mathrm{L}$ in the upper unit of the Chicot aquifer to $337 \mathrm{mg} / \mathrm{L}$ in the lower unit of the Chicot aquifer to $307 \mathrm{mg} / \mathrm{L}$ in the Evangeline aquifer.

\section{SUMMARY AND CONCLUSIONS}

The population of Fort Bend County has increased from about 52,314 in 1969 to 179,732 in 1986. The water needs of the population are supplied by ground water. In 1980, 17 percent of the households obtained water from individual wells and 83 percent from public-supply systems. Of the at least 90 large-capacity wells drilled since 1969, 57 were for public supply. Fifty of these new public supply wells have been drilled in the urban northeast area of the county. These wells average $1,180 \mathrm{ft}$ in depth and have about 80 percent of their screens open to the Evangeline aquifer. In addition, at least 23 large-capacity irrigation wells and 10 large-capacity industrial wells have been drilled in the county since 1969. Eighteen of the new irrigation wells are for rice irrigation. These wells have an average depth of $735 \mathrm{ft}$ and have about 75 percent of their screens open to the Chicot aquifer. The 10 industrial wells drilled since 1969 have an average depth of 
$725 \mathrm{ft}$ and have about 75 percent of their screens open to the Chicot aquifer. of the total 149 large-capacity wells in the county in 1987, 69 were publicsupply wells, 63 were rice-irrigation we1ls, and 17 were industrial wells.

The total withdrawal of ground water increased irregularly from $56 \mathrm{Mgal} / \mathrm{d}$ in 1969 to $72 \mathrm{Mgal} / \mathrm{d}$ in 1982, but then decreased to $53 \mathrm{Mgal} / \mathrm{d}$ in 1986 . Public-supply withdrawals, paralleling the steadily growing population, increased from $4 \mathrm{Mgal} / \mathrm{d}$ in 1969 to $28 \mathrm{Mgal} / \mathrm{d}$ in 1986. About 80 percent of the total public-supply withdrawal came from the northeastern part of the county where most of the new public-supply wells were drilled. In 1969, withdrawal for public supply from the Chicot and Evangeline aquifers was about equal, but in 1986, most of the water withdrawn ( 80 percent) came from the Evangeline aquifer.

Total withdrawal within the county historically has varied with irrigation withdrawal, which, in turn, is related to acres of rice grown. About 90 percent of the irrigated land is planted in rice. During 1969-82 and 1984, an average of 17,500 acres of rice was irrigated by ground water each year, whereas in 1983, 1985, and 1986 the yearly average was 8,700 acres. Groundwater withdrawals for irrigation averaged 38 Mgal/d during 1969-82 and 1984, but only averaged $20 \mathrm{Mgal} / \mathrm{d}$ in 1983, 1985, and 1986. Almost all of the water withdrawn for irrigation ( 80 to 90 percent) has been from the Chicot aquifer.

Industrial withdrawal decreased from $13 \mathrm{Mgal} / \mathrm{d}$ in 1969 to $5 \mathrm{Mgal} / \mathrm{d}$ in 1986. Most of the water withdrawn (70 to 90 percent) has been from the Chicot aquifer. With the increasing withdrawals for public supply and the decreasing withdrawals for irrigation and industry, the principal use of ground water changed from irrigation to public supply. Because of this change, ground water was withdrawn about equally from the Chicot and Evangeline aquifers in 1986. In 1969, most of the water withdrawn ( 85 percent) was from the Chicot aquifer.

Water levels in wells screened in the upper unit of the Chicot aquifer generally have fluctuated less than $4 \mathrm{ft}$ between 1968-69 and 1987, even in areas of large decline in the potentiometric surfaces of the underlying units. The net decline in the potentiometric surface of the lower unit of the Chicot aquifer ranged from less than $10 \mathrm{ft}$ in much of the western part to about 100 $\mathrm{ft}$ in the northeastern part of the county between 1968-69 and 1987. In the southwestern part of the county, the net decline was less than $20 \mathrm{ft}$. Hydrographs indicate that water levels in wells in the lower unit of the Chicot aquifer have either risen a few feet or remained constant after 1982 in much of the county. Water levels in wells screened in the lower unit of the chicot aquifer in the northeastern part of the county have continued to decline. 
The net decline in the potentiometric surface of the Evangeline aquifer ranged from less than $25 \mathrm{ft}$ in the northwestern part to $125 \mathrm{ft}$ in the northeastern part of the county from 1968-69 through 1987. Hydrographs of water levels in wells screened in the Evangeline aquifer in the northeastern part of the county also indicate a recent stabilization of water levels.

The declining water levels have resulted in land-surface subsidence. The subsidence measured by 1978 ranged from more than $0.5 \mathrm{ft}$ in most of the county to more than $2.0 \mathrm{ft}$ in the northeastern part. Studies in the adjacent Houston area showed that most of the compaction occurs in the shallower chicot aquifer and that the compressibility of the clays increases towards the coast. The amount and the percentage of $\mathrm{clay}$ in the Chicot aquifer also increases towards the coast. The total thickness of clay in the Chicot aquifer increases from less than $150 \mathrm{ft}$ near Katy to more than $350 \mathrm{ft}$ in the eastern part of the county. The amount of clay in the Evangeline aquifer also increases towards the coast from an average of about $700 \mathrm{ft}$ near Katy to about 1,100 ft in the eastern part of the county. The compressibility of the clays in Fort Bend County, as indicated by specific-unit compaction values, is similar to that of the inland compaction monitor sites of Addicks, Houston-Northeast, HoustonSouthwest, and Lake Houston. Water-level declines greater than the preconsolidation stress of the clays probably have occurred in the northeastern part of the Fort Bend County, but not in the western and southwestern parts. The northeastern part of the county is the most susceptible to landsurface subsidence because it is in the area with the largest water-level declines and the thickest amount of the most compressible clays.

Concentrations of dissolved constituents in the water from wells have not changed substantially since 1969. Water from the upper unit of the chicot aquifer is very hard and has a median dissolved-solids concentration of $\mathbf{4 7 5}$ $\mathrm{mg} / \mathrm{L}$. Water from the lower unit of the Chicot aquifer is hard and has a median dissolved-solids concentration of $337 \mathrm{mg} / \mathrm{L}$. Water from the Evangeline aquifer is moderately hard and has a median dissolved-solids concentration of $307 \mathrm{mg} / \mathrm{L}$. 


\section{REFERENCES CITED}

Gabrysch, R.K., 1969, Land-surface subsidence in the Houston-Galveston region, Texas: International symposium on land subsidence, Tokyo, Japan, 1969, Proceedings, Publication no. 88, AIHS, p. 43-54.

-.--1972, Development of ground water in the Houston District, Texas 1966-69: Texas Water Development Board Report 152, 24 p.

1977, Approximate areas of recharge to the Chicot and Evangeline aquifer systems in the Houston-Galveston area, Texas: U.S. Geological Survey Open-File Report 77-754, 1 p.

-.--1980, Development of ground water in Houston district, Texas, 1970-74: Texas Department of Water Resources Report 241, 49 p. (also published as U.S. Geological Survey Open-File Report 77-413, 1977).

...-1982, Ground-water withdrawals and land-surface subsidence in the Houston-Galveston region, Texas, 1906-80: U.S. Geological Survey OpenFile Report 82-571, 68 p. (also published as Texas Department of Water Resources Report 287, 1984).

-.--1984, Ground-water withdrawals and changes in water levels in the Houston district, Texas, 1975-79: Texas Department of Water Resources Report 286, 42 p. (also published as U.S. Geological Survey Open-File Report $82-431,1982)$.

Gabrysch, R.K., and Bonnet, C.W., 1975, Land-surface subsidence in the Houston-Galveston region, Texas: Texas Water Development Board Report. 188, 19 p. (also published as U.S. Geological Survey Open-File Report $74-123,1974)$.

Holzer, T.L., 1981, Preconsolidation stress of aquifer systems in areas of induced land subsidence: Water Resources Research, v. 17, no. 3, p. 693704.

Holzer, T.L., and Gabrysch, R.K., 1987, Effect of water-level recoveries on fault creep, Houston, Texas: Ground Water, v. 25, no. 4, p. 392-397.

Jorgensen, D.G., 1975, Analog-model studies of ground-water hydrology in the Houston district, Texas: Texas Water Development Board Report 190, 84 p. (also published as U.S. Geological Survey Open-File Report 74-113, 1974).

Jorgensen, D.G., 1981, Geohydrologic models of the Houston District, Texas: Ground Water, v. 19, no. 4, p. 418-428.

Lang, J.W., Winslow, A.G., and White, W.N., 1950, Geology and ground-water resources of the Houston district, Texas: Texas Board of Water Engineers Bulletin 5001, $55 \mathrm{p}$.

Loskot, C.L., Sandeen, W.M., and Follett, C.R., 1982, Ground-water resources of Colorado, Lavaca, and Wharton Counties, Texas: Texas Department of Water Resources Report 270, 245 p. (also published as U.S. Geological Survey Open-File Report 79-212, 1979). 


\section{REFERENCES CITED--Continued}

Naftel, W.L., Vaught, Kenneth, and Fleming, Bobbie, 1976, Records of wells, drillers' logs, water-level measurements, and chemical analyses of ground water in Brazoria, Fort Bend, and Waller Counties, Texas, 196674: Texas Water Development Board Report 201, $90 \mathrm{p}$.

Ratzlaff, K.W., Ranzau, C.E., and Lind, W.B., 1983, Records of wells, drillers' logs, water-level measurements, and chemical analyses of ground water in Brazoria, Fort Bend, and Waller Counties, Texas, $1975-$ 79: Texas Department of Water Resources Report 277, $57 \mathrm{p}$.

Sandeen, W.M., and Wesselman, J.B., 1973, Ground-water resources of Brazoria County, Texas: Texas Water Development Board Report 163, $199 \mathrm{p}$.

Texas A\&M University, 1986, Texas population estimates, county place totals: Texas A\&M University, Department of Rural Sociology, $43 \mathrm{p}$.

Texas Department of Health (eds.), 1983, Chemical analyses of public water systems, $135 \mathrm{p}$.

Texas Water Development Board, 1975, Inventories of irrigation in Texas, 1958, 1964, 1969, and 1974: Texas Water Development Board Report 196, 265 p. -1986, Surveys of irrigation in Texas, 1958, 1964, 1969, 1974, 1979, and 1984: Texas Water Development Board Report 294, 243 p.

U.S. Bureau of the Census, 1972, Census of housing: 1970, Housing characteristics for States, cities, and counties, v. 1, chapter B, part 45, Detailed housing characteristics: U.S. Government Printing Office.

-..-1973, Census of population: 1970, Characteristics of the population, v. 1, part 45, Texas: U.S. Government Printing office

1983, Census of housing: 1980, Characteristics of housing units, v. 1, chapter B, part 45, Detailed housing characteristics: U.S. Government Printing office.

Wesselman, J.B., 1972, Ground-water resources of Fort Bend County, Texas: Texas Water Development Board Report 155, $185 \mathrm{p}$.

Williams, J.F., III, Lind, W.B., and Copl in, L.S., 1987, Approximate altitude of water levels in wells in the Chicot and Evangeline aquifers in the Houston area, Texas, Spring 1987: U.S. Geological Survey Open-File Report 87-233, 2 sheets.

Williams, J.F., III, and Ranzau, C.E., Jr., 1987, Ground-water withdrawals and changes in ground-water levels, ground-water quality, and land-surface subsidence in the Houston district, Texas, 1980-84: U.S. Geological Survey Water Resources Investigations Report 87-4153, $56 \mathrm{p}$.

Williams, J.F., III, Ranzau, C.E., Jr., and Lind, W.B., 1987, Approximate water-level changes in wells completed in the Chicot and Evangeline aquifers, 1977-87 and 1986-87, and measured compaction, 1973-87, in the Houston-Galveston region, Texas: U.S. Geological Survey Open-File Report $87-108,7$ sheets. 


\section{REFERENCES CITED--Continued}

Williams, J.F., III, Ranzau, C.E., Jr., Lind, W.B., and Coplin, L.S., 1986, Records of wells, drillers' logs, water-level measurements, and chemical analyses of ground water in Brazoria, Fort Bend, and Waller Counties, Texas, 1980-84: U.S. Geological Survey Open-File Report 86-068, 51 p.

Wilson, C.A., 1967, Ground-water resources of Austin and Waller Counties, Texas: Texas Water Development Board Report 068, $231 \mathrm{p}$.

Wood, L.A., and Gabrysch, R.K., 1965, Analog model study of ground water in the Houston District, Texas: Texas Water Commission Butletin 6508, $103 \mathrm{p}$.

Wood, L.A., Gabrysch, R.K., and Marvin, Richard, 1963, Reconnaissance investigation of the ground-water resources of the Gulf Coast region, Texas: Texas Water Commission Bulletin 6305, $114 \mathrm{p}$. 
SUPPLEMENTAL DATA

$-40-$ 
Table 1.--Correlation of geologic and hydrologic units

(Modified from Williams and Ranzau, 1987)

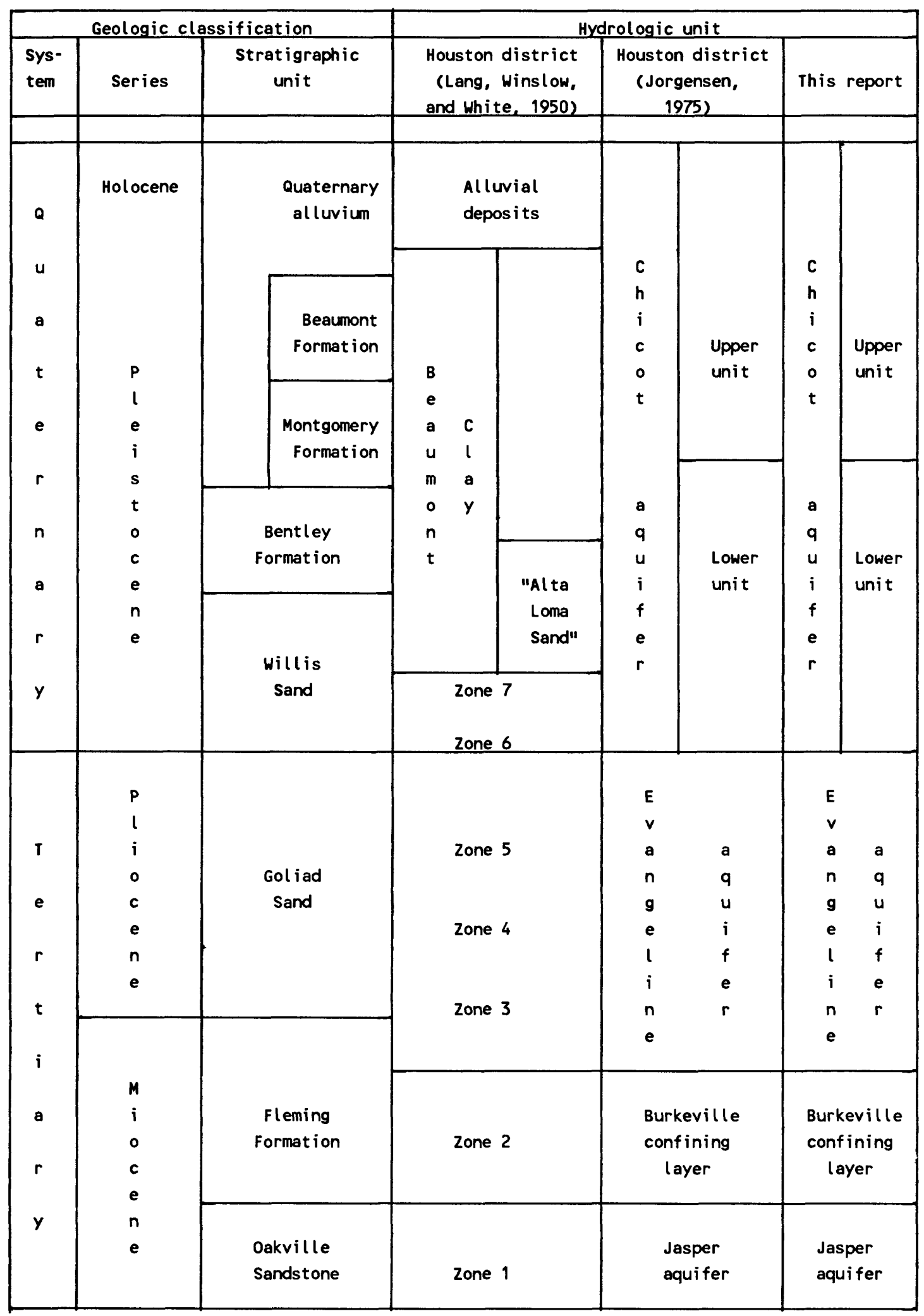




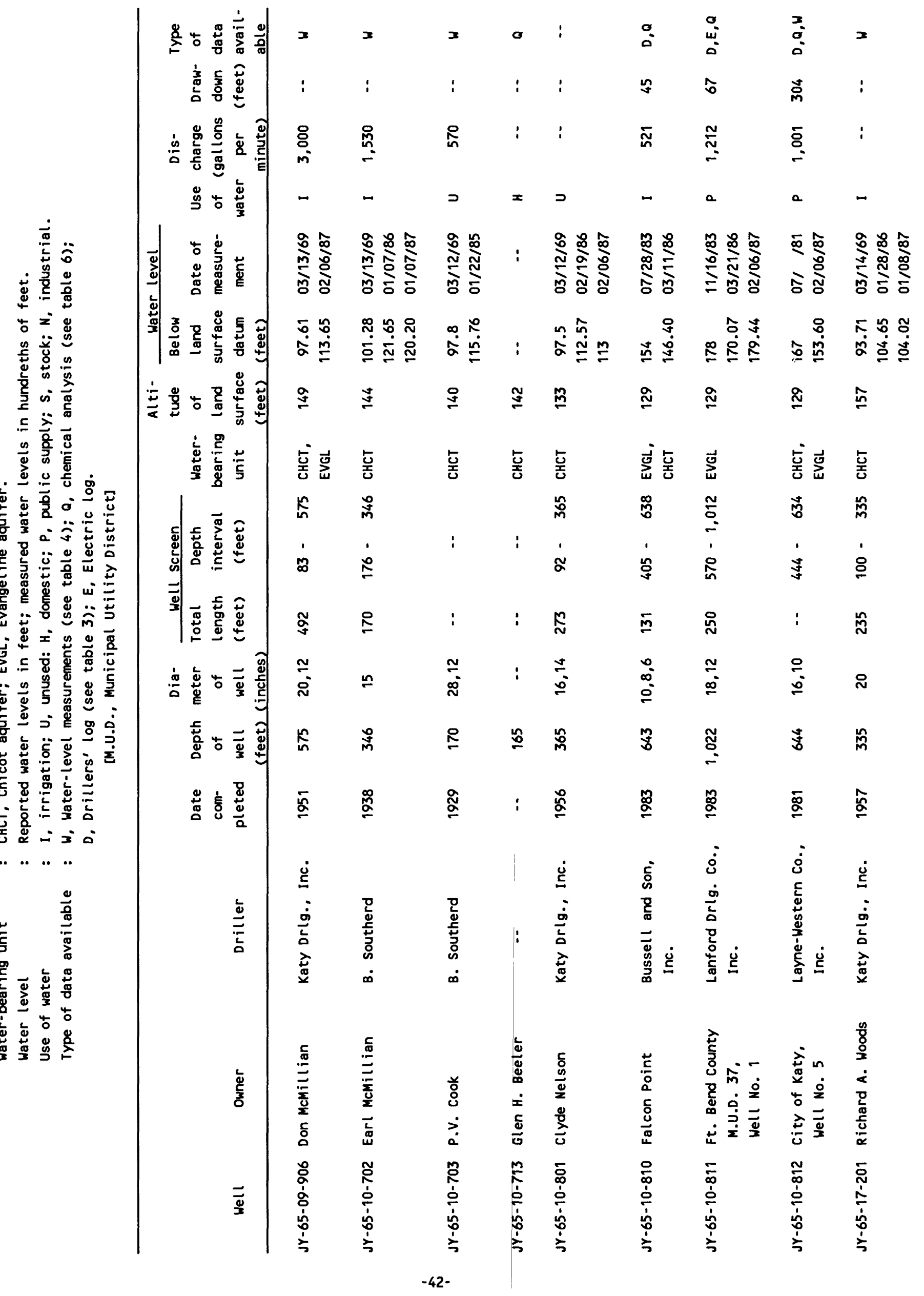




\begin{tabular}{|c|c|c|c|c|c|c|c|c|c|c|c|c|}
\hline 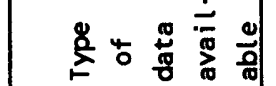 & o & $=$ & $\stackrel{8}{\circ}$ & : & 0 & : & : & : & $=$ & . & . & 0 \\
\hline 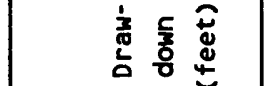 & $:$ & : & n & : & ฉ̊ & : & : & : & : & : & స̃ & : \\
\hline 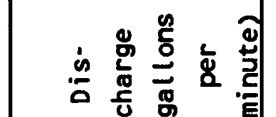 & $:$ & $:$ & $\underset{\substack{\tilde{a} \\
\dot{m}}}{ }$ & $\underset{\substack{o \\
i}}{i}$ & 商 & : & 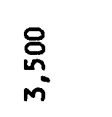 & : & $\stackrel{\varrho}{\varrho}$ & : & $\stackrel{8}{g}$ & $\stackrel{8}{2}$ \\
\hline 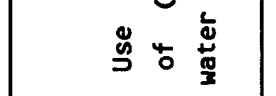 & - & - & - & - & - & o & $=$ & D & o & a & - & $x$ \\
\hline 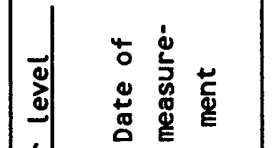 & $:$ & 商产 & 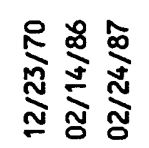 & 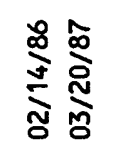 & $\frac{0}{\stackrel{0}{0}}$ & 总 & 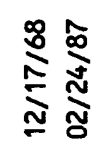 & 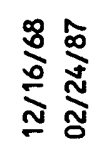 & 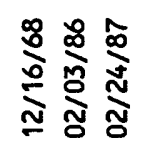 & 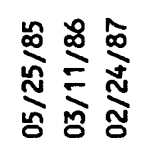 & $\begin{array}{l}\cong \\
\equiv\end{array}$ & 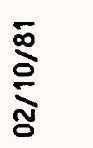 \\
\hline 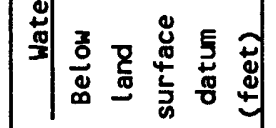 & $:$ & 魚 & 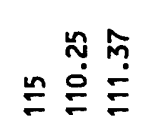 & 㝵号 & $\nsubseteq$ & $\stackrel{\square}{\dot{g}}$ & $\stackrel{8}{9}$ & 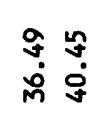 & 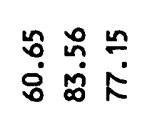 & 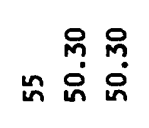 & $\cong$ & is \\
\hline 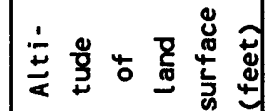 & $\stackrel{\infty}{\check{0}}$ & $\underline{n}$ & $\underline{n}$ & $\Xi$ & $\cong$ & \pm & $\stackrel{ \pm}{ \pm}$ & $\cong$ & \pm & $\stackrel{\circ}{\circ}$ & $\cong$ & $\stackrel{\text { L }}{\underline{\theta}}$ \\
\hline 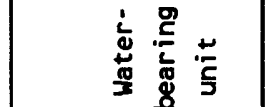 & 厗 & 产 & 寡总 & 瞎 总 & 瞎 崽 & $\stackrel{\breve{z}}{\underline{\underline{t}}}$ & 喜 & 产 & 喜 & $\stackrel{\underline{\underline{I}}}{\underline{\underline{y}}}$ & 寡 总 & 홇 \\
\hline & & & $\stackrel{\sim}{\mathfrak{m}^{\circ}}$ & $\hat{0}$ & $\bar{\hbar}$ & & $\stackrel{\infty}{m}$ & ồ & & g & $\tilde{D}$ & ळ్ \\
\hline 氮 & $:$ & $:$ & $\stackrel{\circ}{\circ}$ & $\tilde{\tilde{n}}$ & $\Phi$ & : & $\dot{\infty}$ & $\cong$ & : & ๙ิ & i & $\stackrel{8}{N}$ \\
\hline 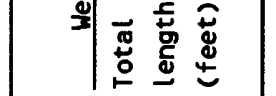 & $:$ & $:$ & $\widehat{\Im}$ & 兽 & 8 & $:$ & $\stackrel{2}{2}$ & $\stackrel{8}{\circ}$ & $:$ & ळ & $\%$ & న \\
\hline 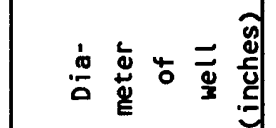 & $\stackrel{\sim}{2}$ & i & $\stackrel{\sim}{\simeq}$ & $\frac{n}{i}$ & $\frac{n}{i}$ & $\checkmark$ & i & $\stackrel{\simeq}{\circ}$ & 。 & $\stackrel{\circ}{:}$ & $\stackrel{n}{i}$ & $\stackrel{4}{\circ}$ \\
\hline 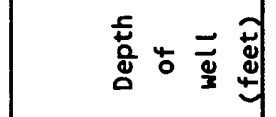 & $\tilde{\tilde{m}}$ & 品 & $\mathscr{m}$ & $\hat{\bar{o}}$ & $\bar{\Sigma}$ & $\stackrel{\circ}{\circ}$ & 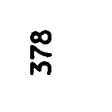 & 总 & $\stackrel{8}{=}$ & $\ddot{g}$ & $\tilde{\varpi}$ & $\stackrel{్}{\sim}$ \\
\hline 吉言 & $\underline{\hat{\alpha}}$ & $\stackrel{q}{2}$ & $\stackrel{2}{g}$ & ๕ & $\stackrel{\circ}{\circ}$ & $:$ & $\underline{\tilde{Q}}$ & $\stackrel{\circ}{\varrho}$ & $\stackrel{\text { g }}{\varrho}$ & $\stackrel{\leftrightarrow}{\circ}$ & $\tilde{\Xi}$ & $\stackrel{\bar{\Phi}}{\mathscr{D}}$ \\
\hline & 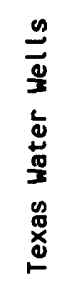 & $\begin{array}{l}\frac{0}{8} \\
\frac{0}{3} \\
\dot{\alpha} \\
\frac{0}{0} \\
\frac{0}{0} \\
\frac{6}{\alpha}\end{array}$ & 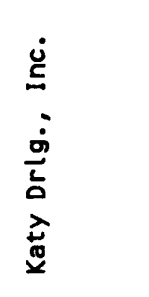 & 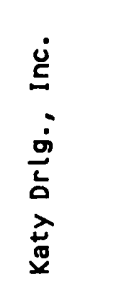 & 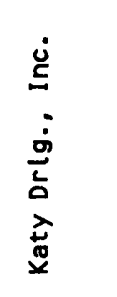 & : & 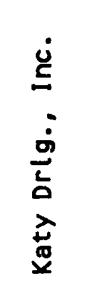 & 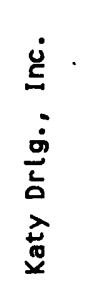 & 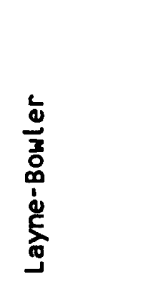 & 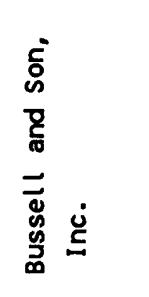 & 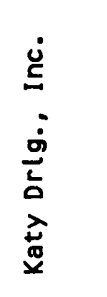 & 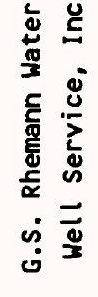 \\
\hline 誩 & 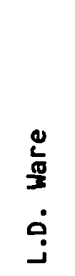 & 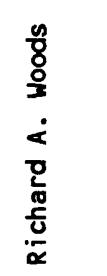 & 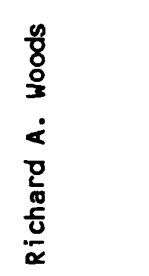 & 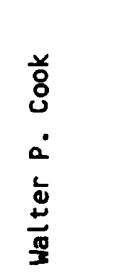 & 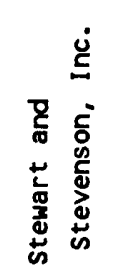 & 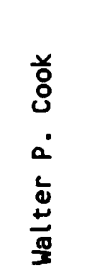 & 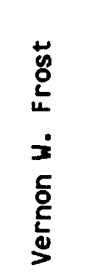 & 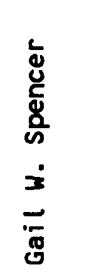 & 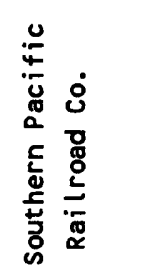 & 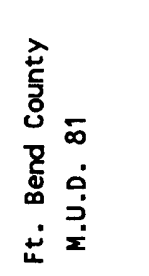 & 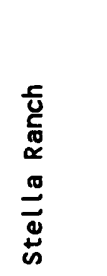 & \\
\hline$\overline{\underline{\underline{D}}}$ & 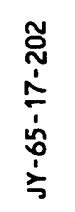 & 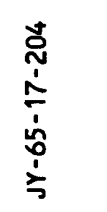 & 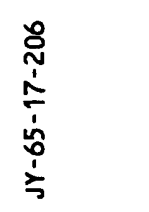 & 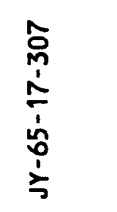 & $\begin{array}{l}\infty \\
\stackrel{0}{0} \\
\stackrel{5}{1} \\
\stackrel{0}{0} \\
\grave{3}\end{array}$ & 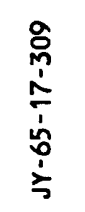 & 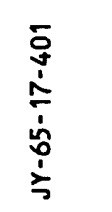 & 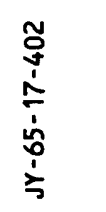 & 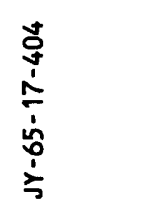 & 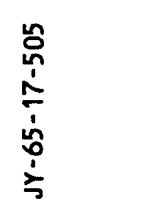 & 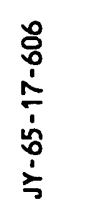 & 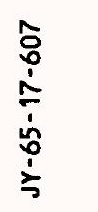 \\
\hline
\end{tabular}




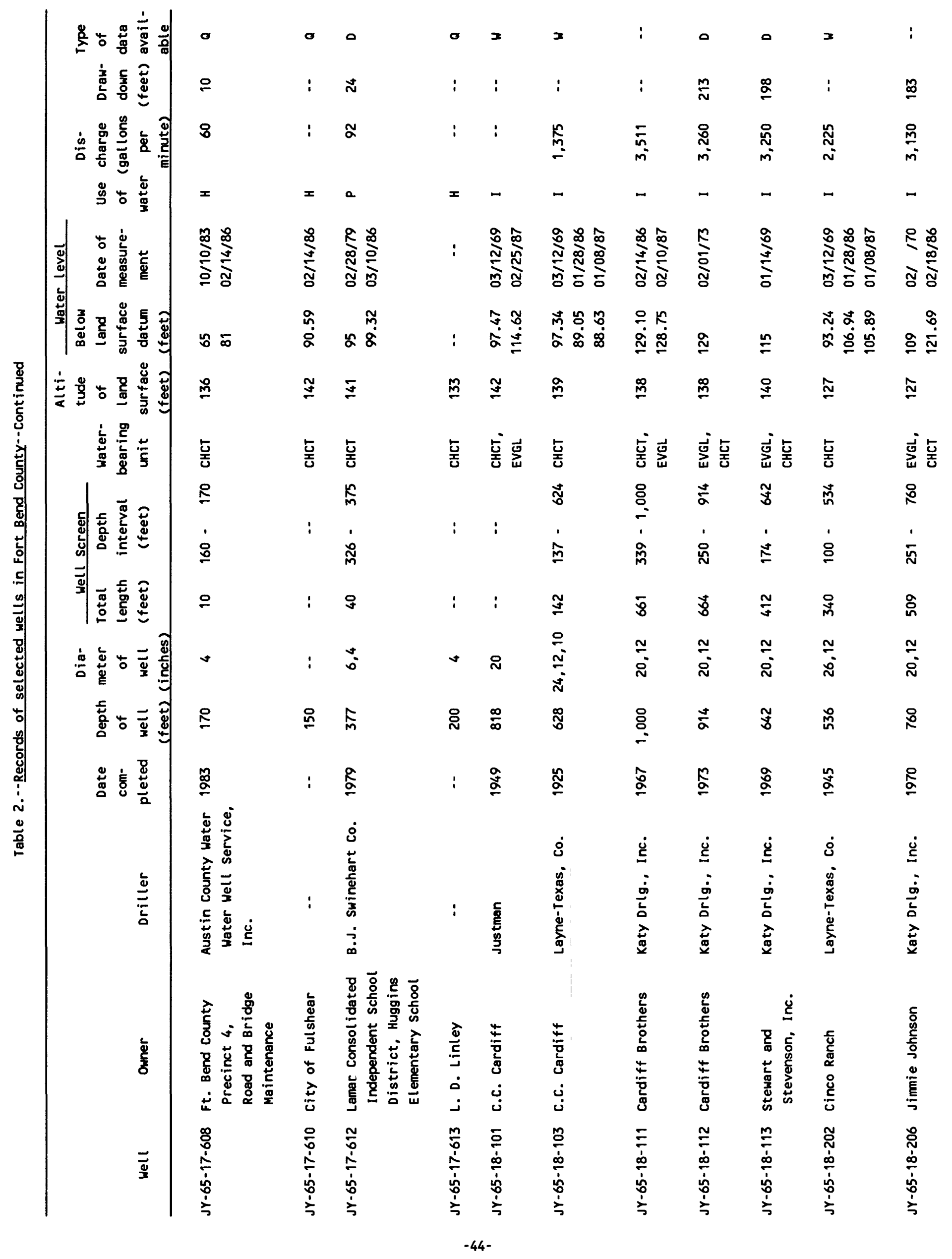




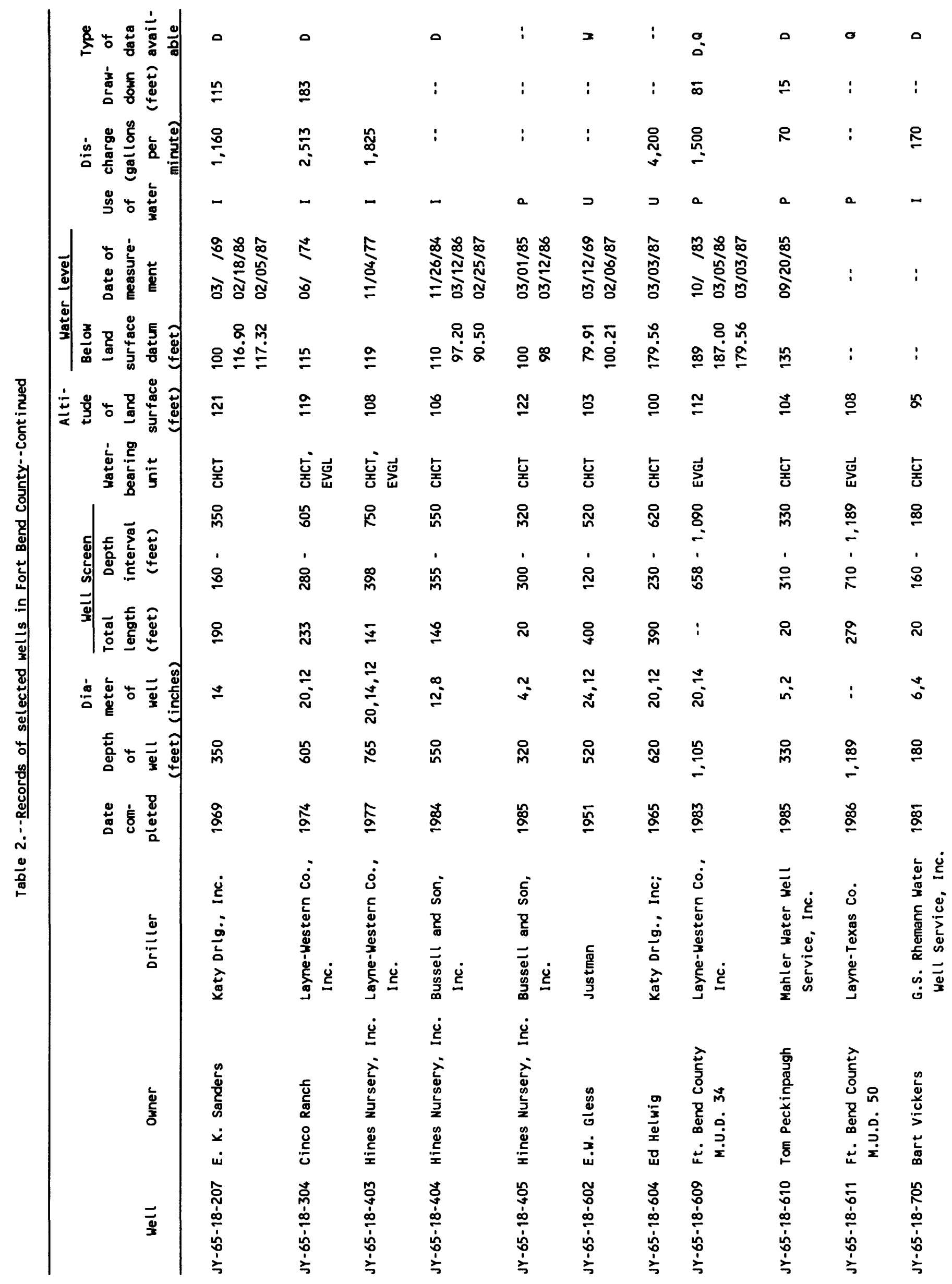




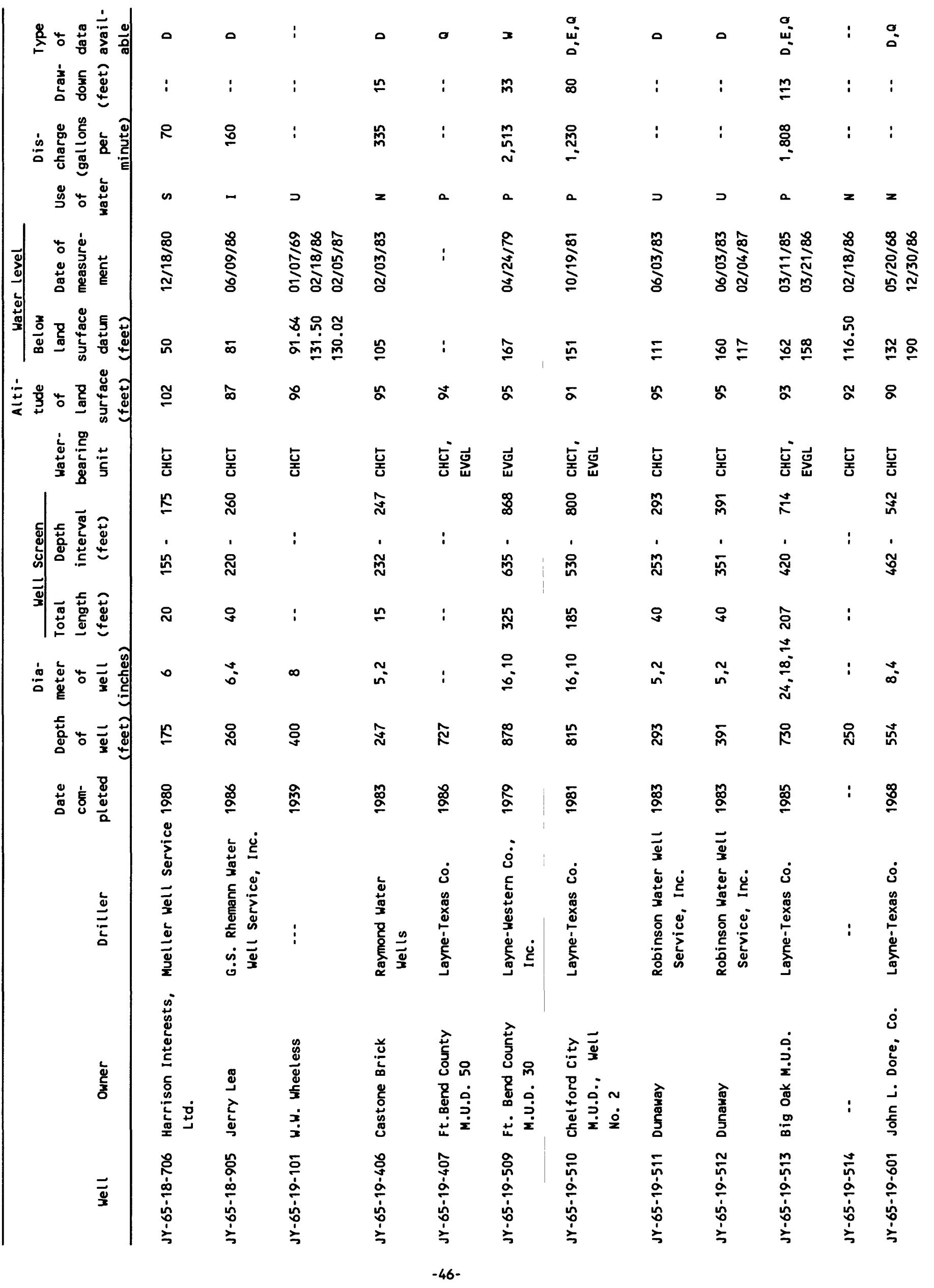




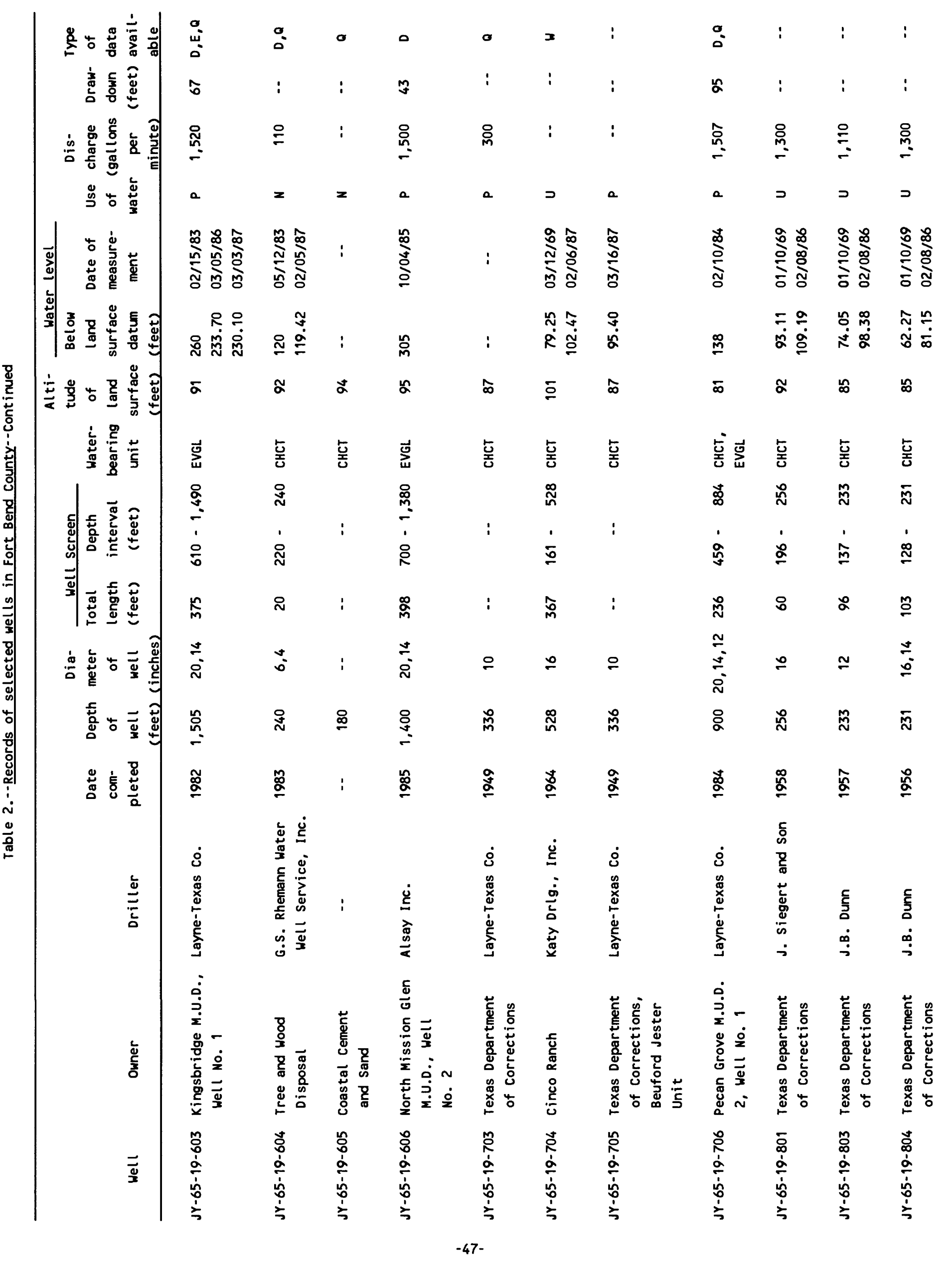




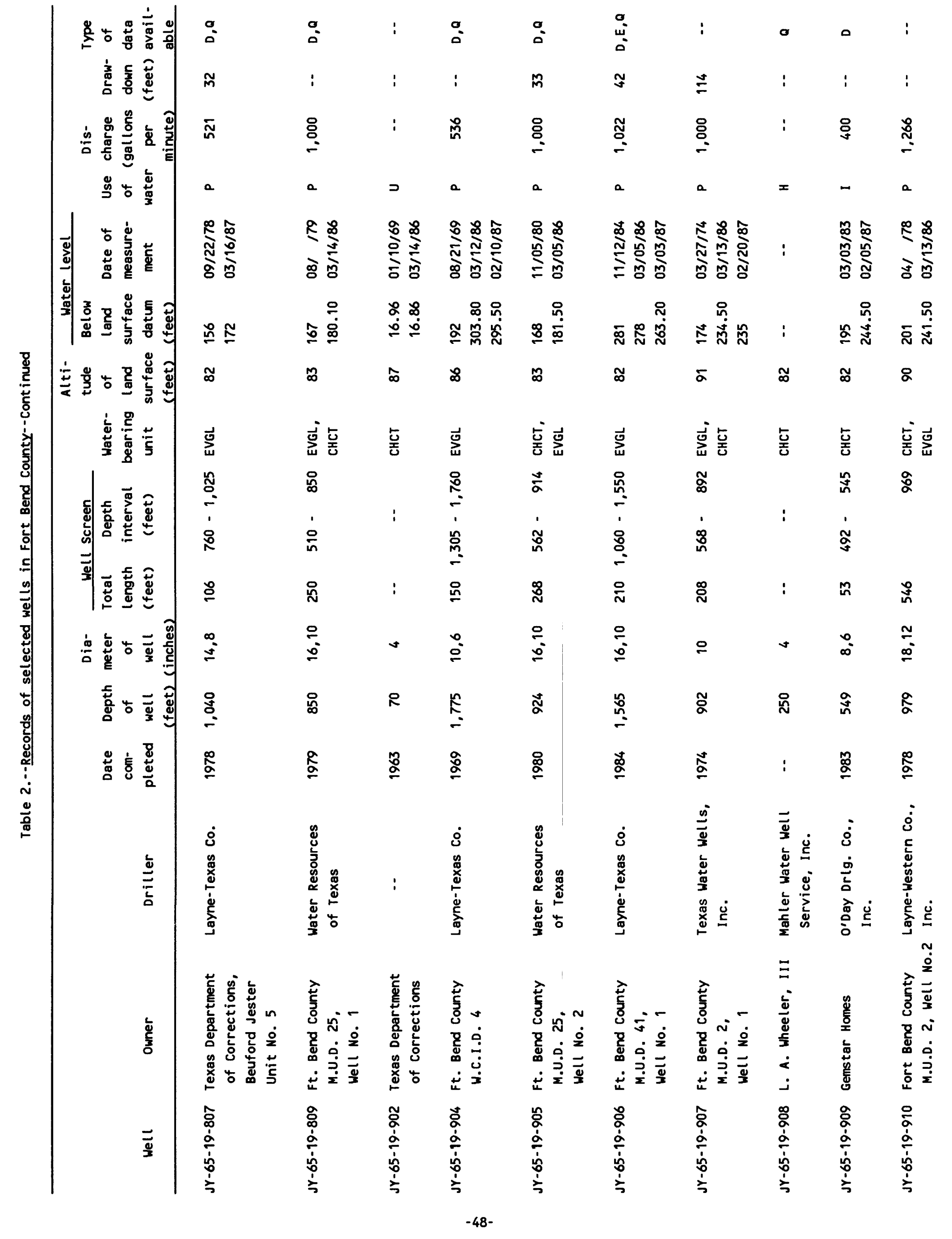




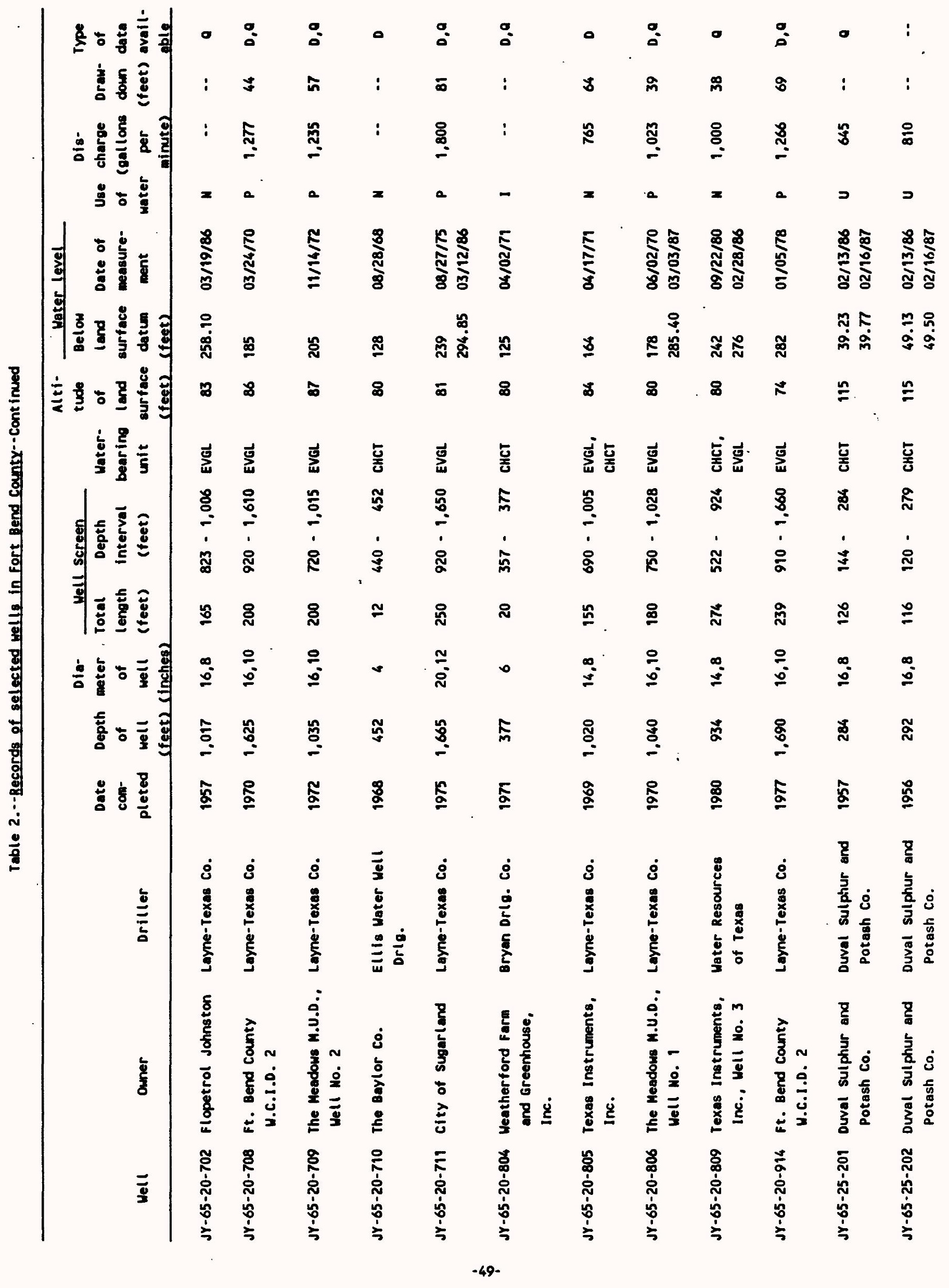




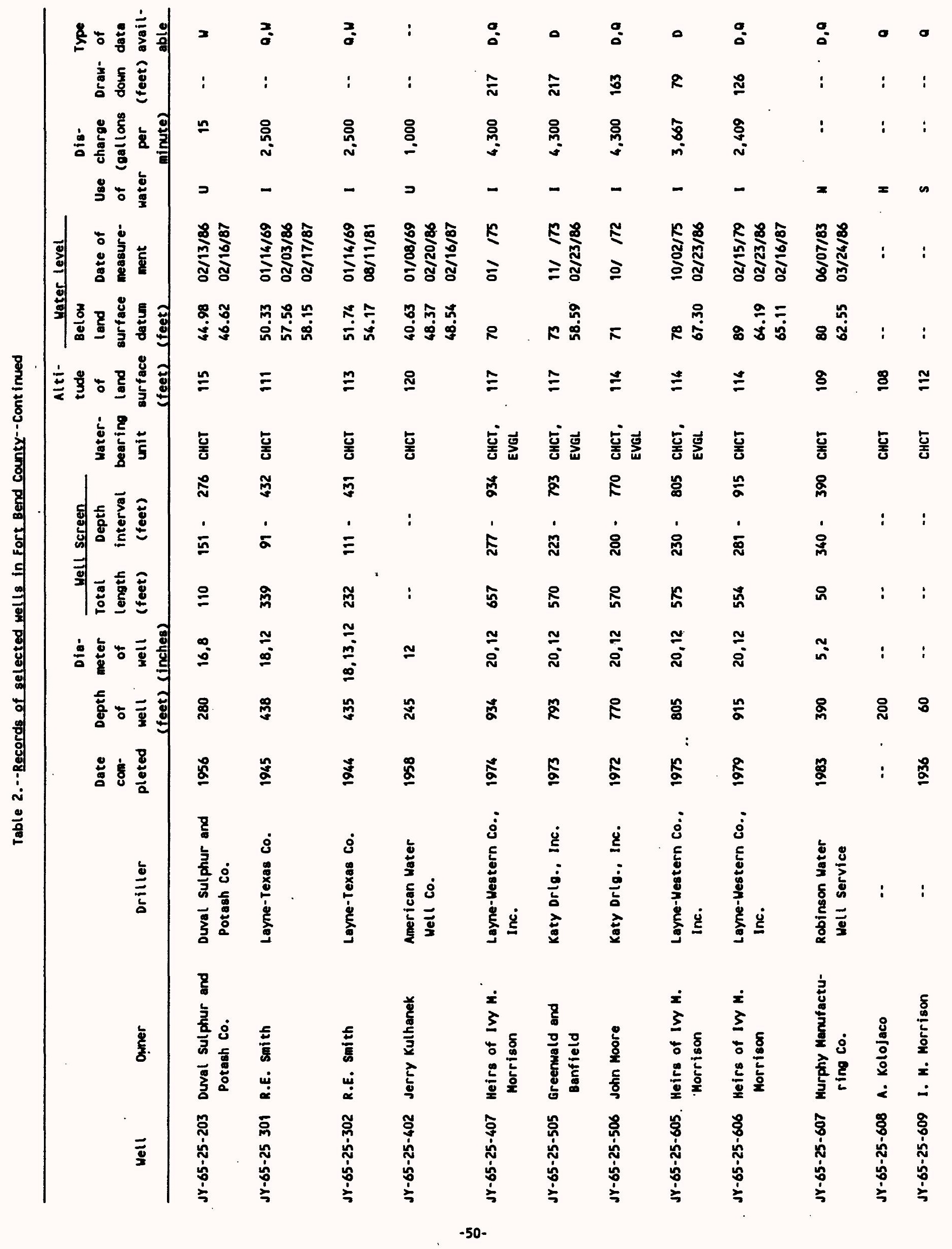




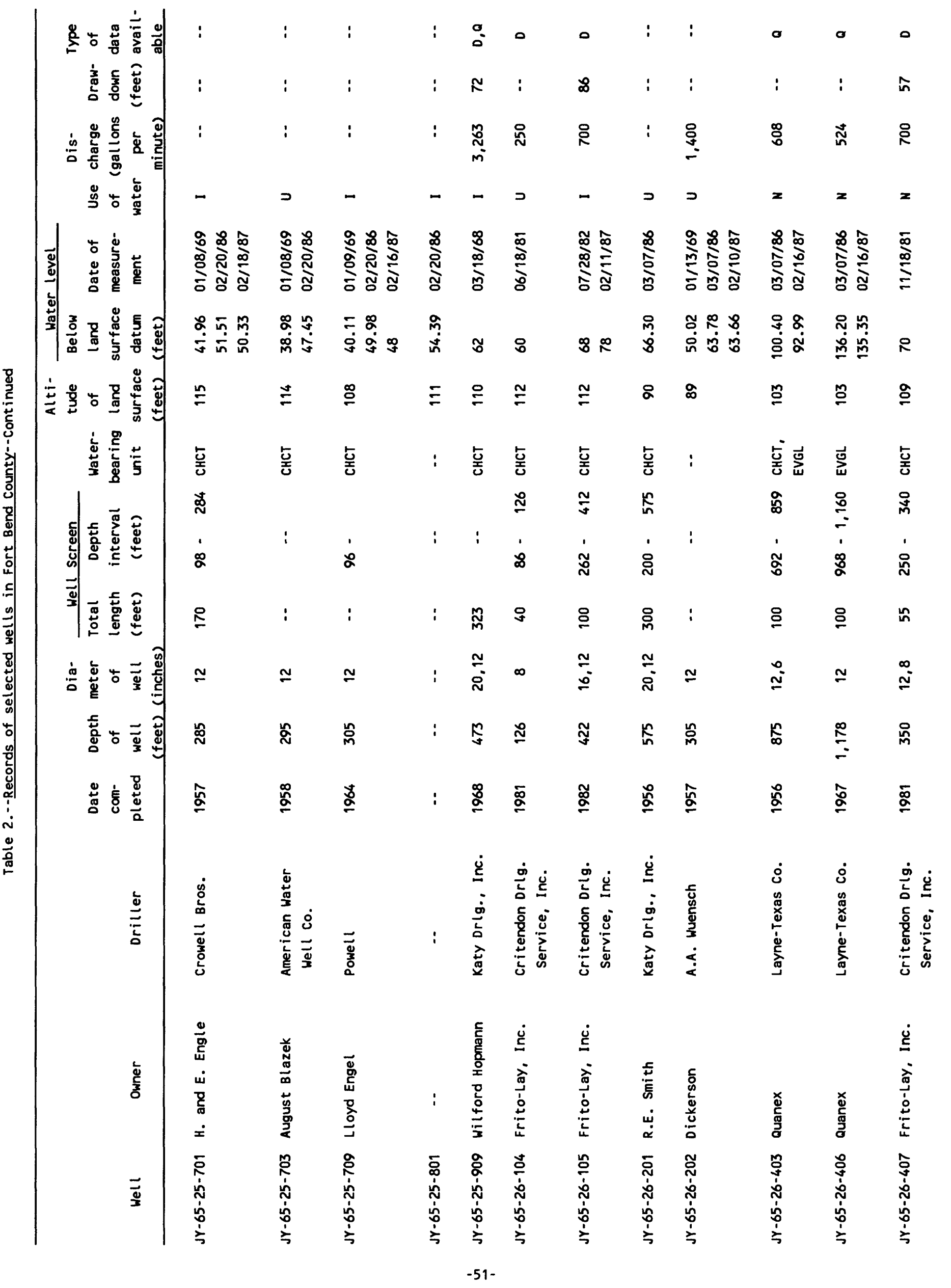




\begin{tabular}{|c|c|c|c|c|c|c|c|c|c|c|c|}
\hline 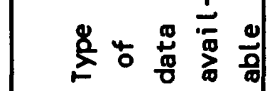 & 0 & 0 & 0 & 0 & 0 & 0 & $\stackrel{a}{\circ}$ & 0 & 0 & 0 & 0 \\
\hline 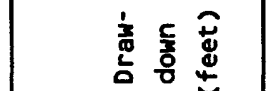 & $F$ & $:$ & $:$ & $:$ & $:$ & $:$ & $\approx$ & $:$ & $:$ & $:$ & $\approx$ \\
\hline 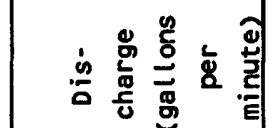 & 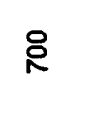 & $\mathscr{N}$ & R & $\underset{\sim}{\tilde{N}}$ & $\stackrel{\substack{m \\
:}}{=}$ & $\stackrel{\widetilde{\Xi}}{=}$ & 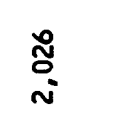 & $\stackrel{8}{\circ}$ & $\cong$ & $\stackrel{\text { O⿱ }}{-}$ & 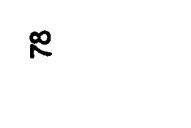 \\
\hline 50 & $=$ & a & a & a & a & a & a & - & $=$ & a & a \\
\hline 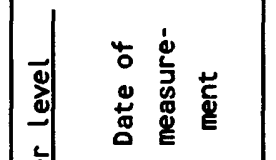 & 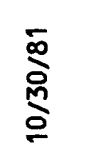 & 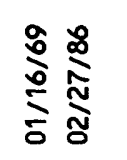 & 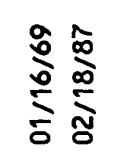 & 产 & 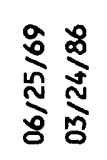 & 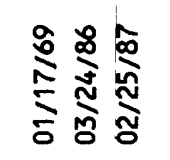 & 果雚 & 趁 & $\stackrel{\stackrel{M}{0}}{\stackrel{\stackrel{M}{I}}{\Xi}}$ & 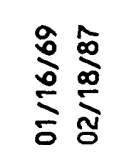 & 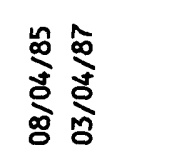 \\
\hline : & 8 & 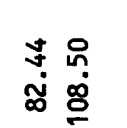 & 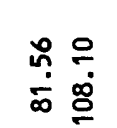 & $\frac{\circ}{8}$ & 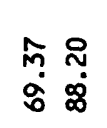 & مُ & 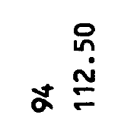 & 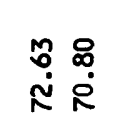 & $\underline{0}$ & 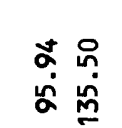 & $\stackrel{\mathscr{n}}{\stackrel{\mathscr{\omega}}{\infty}}$ \\
\hline 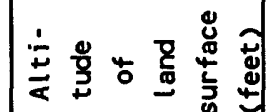 & $\stackrel{8}{g}$ & $\ddot{g}$ & $\stackrel{0}{0}$ & $\dddot{q}$ & ณ & 2 & $\approx$ & $\approx$ & ஃ & 8 & $\approx$ \\
\hline & 厗 & 瞎总 & 瞎喜 & 喜 & $\stackrel{\underline{\underline{s}}}{\underline{s}}$ & 预 & 喜 & 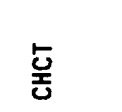 & 喜 & 喜 & 总 \\
\hline & 品 & 第 & 8 & 总 & $f$ & in & 㽞 & $\tilde{n}$ & 苟 & 品 & 品 \\
\hline 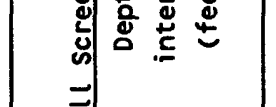 & 总 & 密 & ชิ & 8 & $\hat{n}$ & $\stackrel{\sim}{m}$ & m & $\approx$ & 总 & $\stackrel{\dot{\circ}}{\circ}$ & 怘 \\
\hline 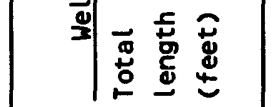 & in & $\stackrel{\sim}{\simeq}$ & 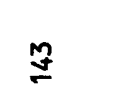 & ఎ & i & $\stackrel{\circ}{m}$ & $\tilde{\text { N }}$ & $\cong$ & $\approx$ & $\stackrel{\text { ڤ }}{\sigma}$ & $\approx$ \\
\hline 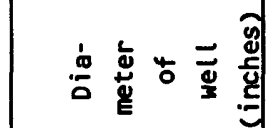 & $\stackrel{\infty}{\sim}$ & $\stackrel{\infty}{\stackrel{\infty}{\infty}}$ & $\stackrel{\infty}{\stackrel{\infty}{ \pm}}$ & $\stackrel{\circ}{\circ}$ & $\stackrel{\infty}{\dot{\infty}}$ & $\stackrel{\circ}{\circ}$ & $\stackrel{\circ}{\circ}$ & $\simeq$ & $\underset{\substack{j \\
\dot{j} \\
0}}{0}$ & $\simeq$ & $\tilde{\sigma}$ \\
\hline 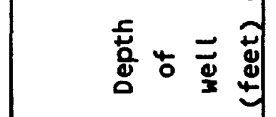 & 品 & 电 & $\frac{2}{2}$ & 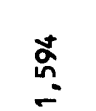 & $\stackrel{\infty}{乛}$ & $\frac{\infty}{n}$ & $\dddot{W}$ & חั & 获 & $\stackrel{m}{n=}$ & 商 \\
\hline 站言 & $\stackrel{\bar{g}}{\circ}$ & $\hat{g}$ & פू & 参 & $\underline{\underline{g}}$ & $\underline{\alpha}$ & $\stackrel{\infty}{\varrho}$ & $\stackrel{\check{\Phi}}{\stackrel{0}{0}}$ & $\stackrel{\Xi}{\mathscr{m}}$ & o & $\stackrel{\ddot{m}}{\mathscr{\Xi}}$ \\
\hline & 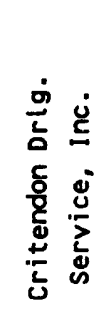 & 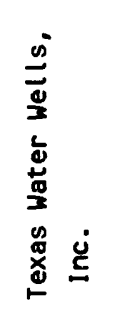 & 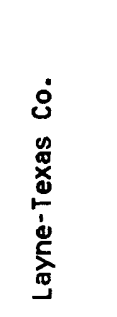 & 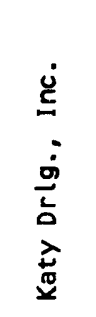 & $\begin{array}{l}\frac{c}{5} \\
\frac{5}{10} \\
\frac{8}{8}\end{array}$ & 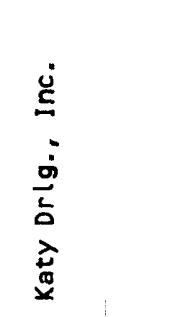 & 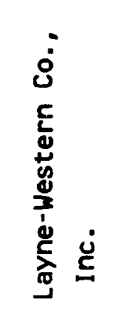 & 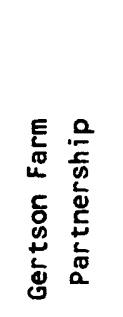 & 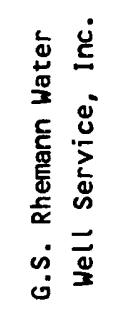 & 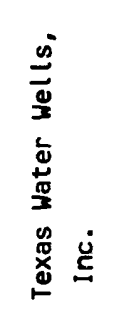 & 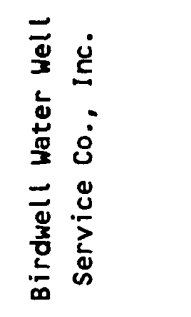 \\
\hline 譬 & 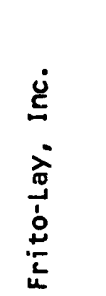 & 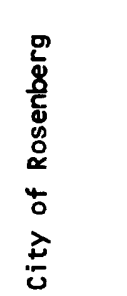 & 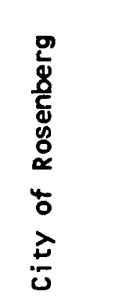 & 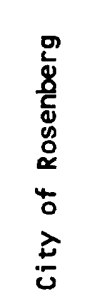 & 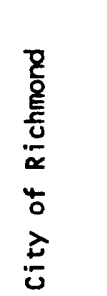 & 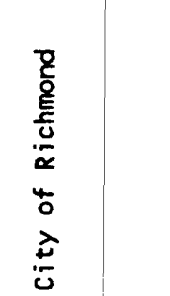 & 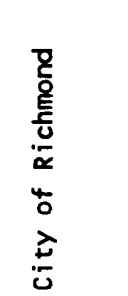 & 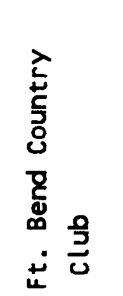 & 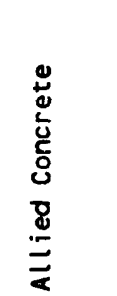 & 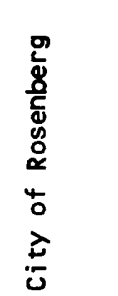 & 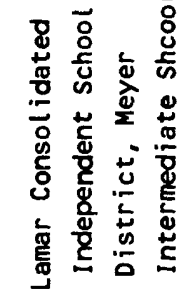 \\
\hline$\overline{\overrightarrow{\mathbf{a}}}$ & 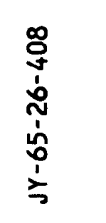 & 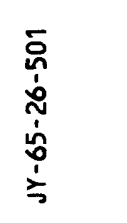 & 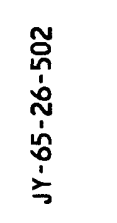 & 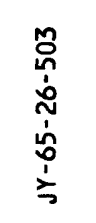 & 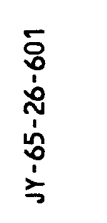 & $\begin{array}{l}\text { m. } \\
\dot{0} \\
\dot{\omega} \\
\dot{\omega} \\
\dot{0} \\
\grave{j}\end{array}$ & 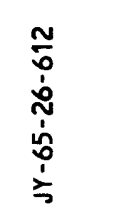 & 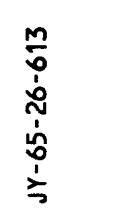 & 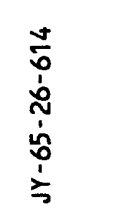 & 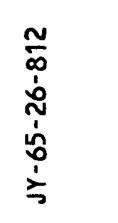 & 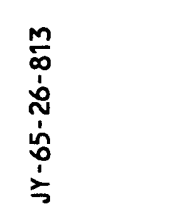 \\
\hline
\end{tabular}




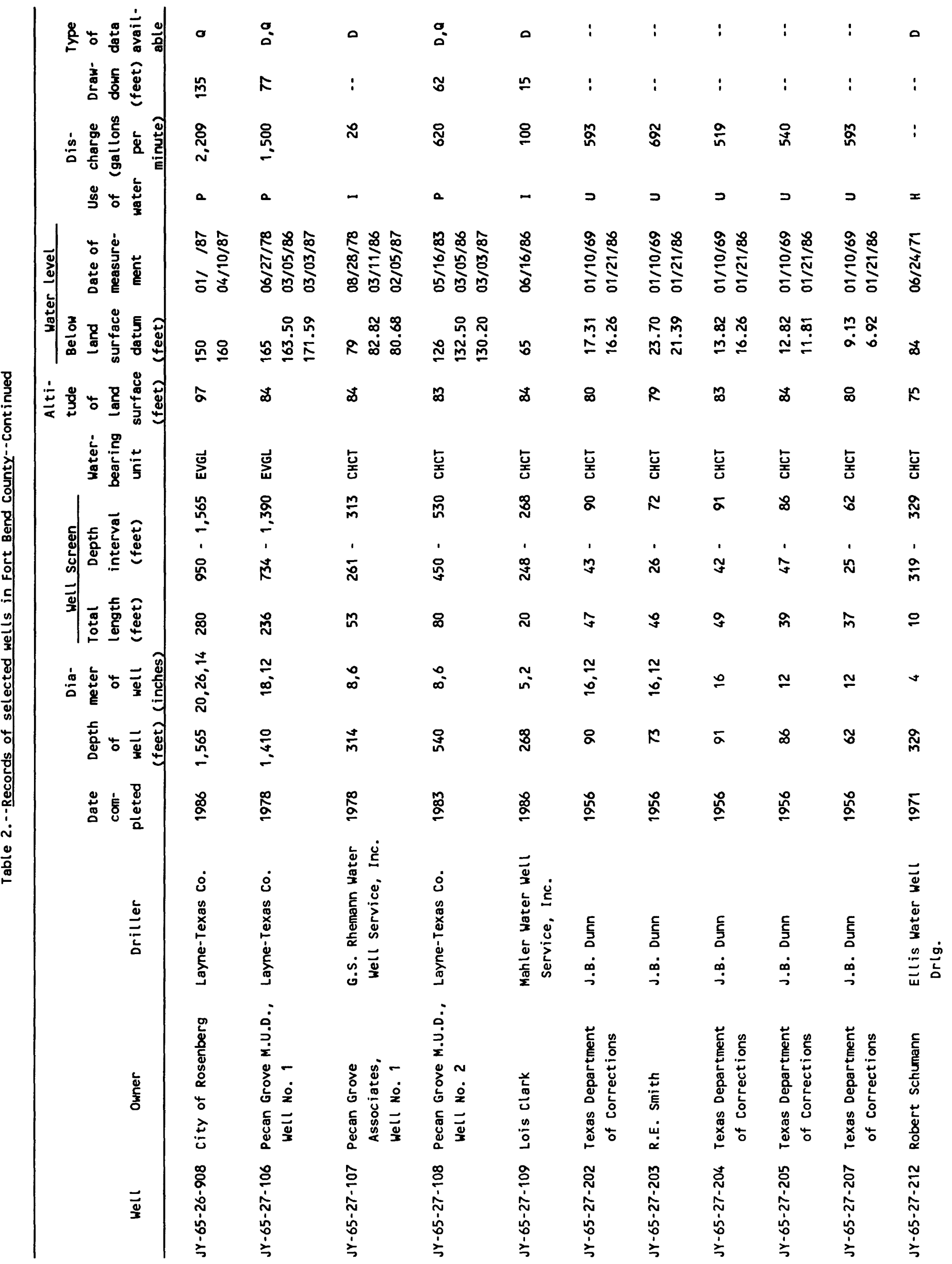




\begin{tabular}{|c|c|c|c|}
\hline 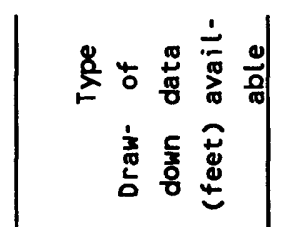 & 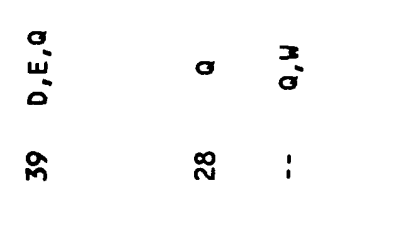 & & $\frac{3}{\partial}=$ \\
\hline 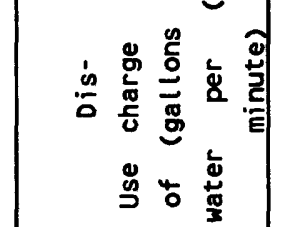 & 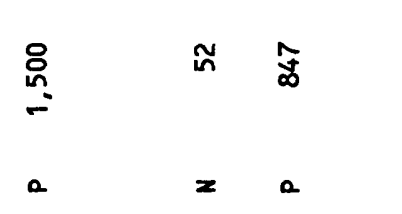 & 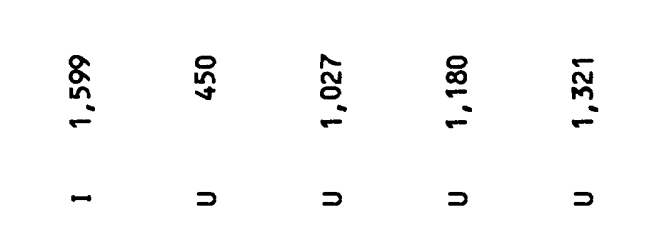 & $: \frac{5}{3}$ \\
\hline & & 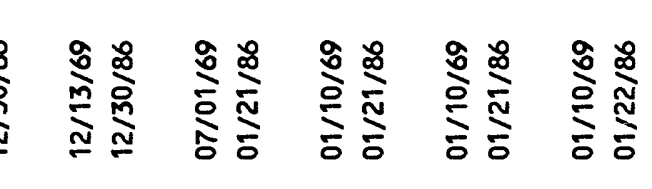 & \\
\hline 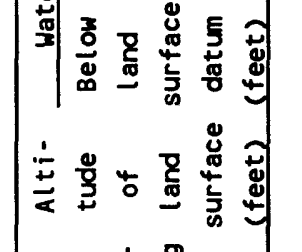 & & 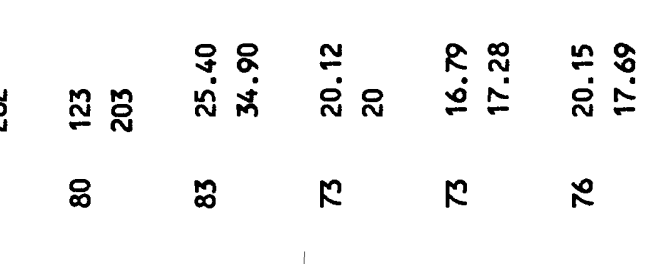 & \\
\hline 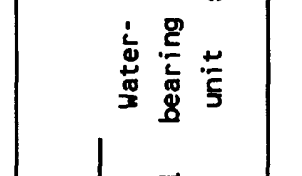 & $\frac{1}{5}$ & 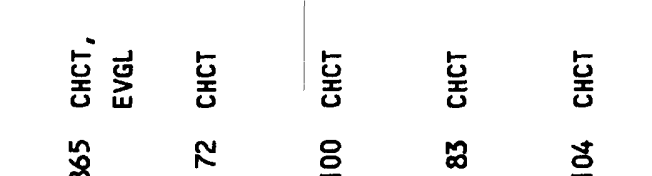 & \\
\hline $\bar{m}$ & 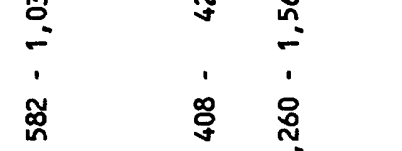 & 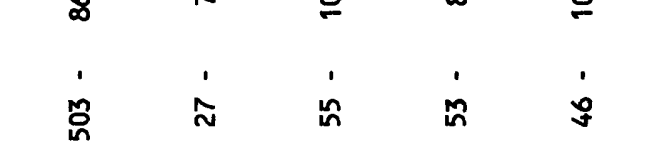 & \\
\hline 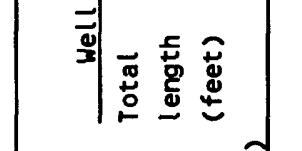 & 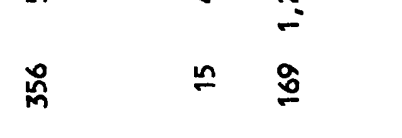 & 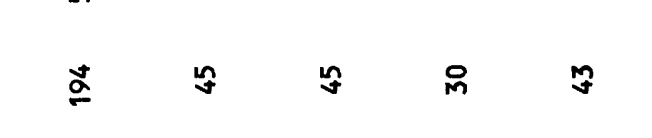 & \\
\hline 的氮 & & $\ddot{z}=2$ & \\
\hline 彭㕸言 & 9 & 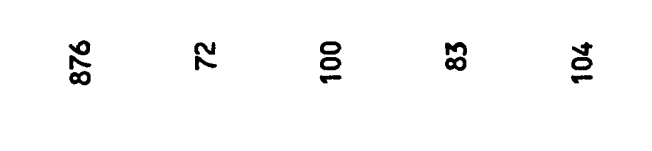 & \\
\hline 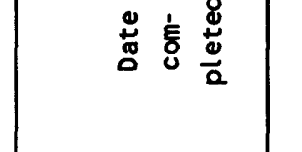 & 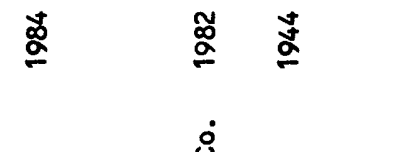 & $\frac{2}{3}$ & \\
\hline & $\frac{1}{2 !}$ & 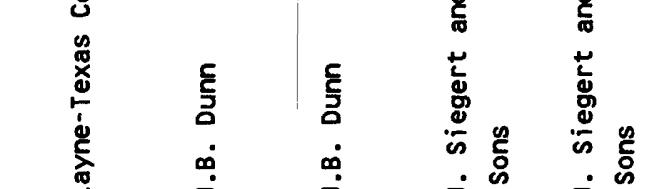 & \\
\hline & $\frac{1}{2}$ & 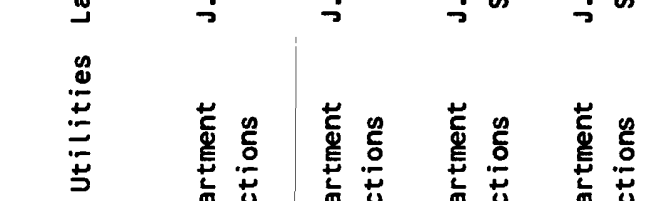 & \\
\hline & & & \\
\hline & & & \\
\hline
\end{tabular}




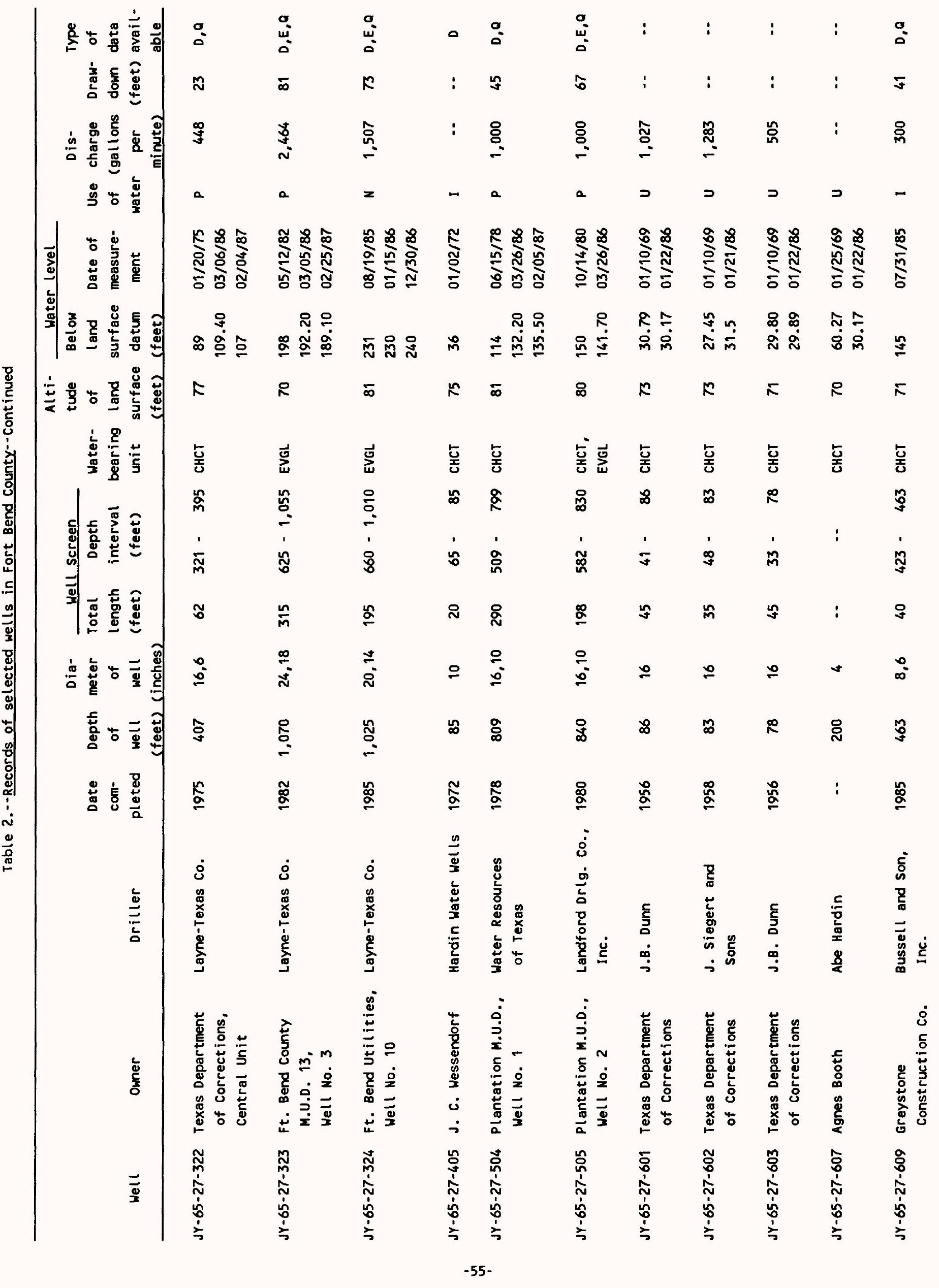




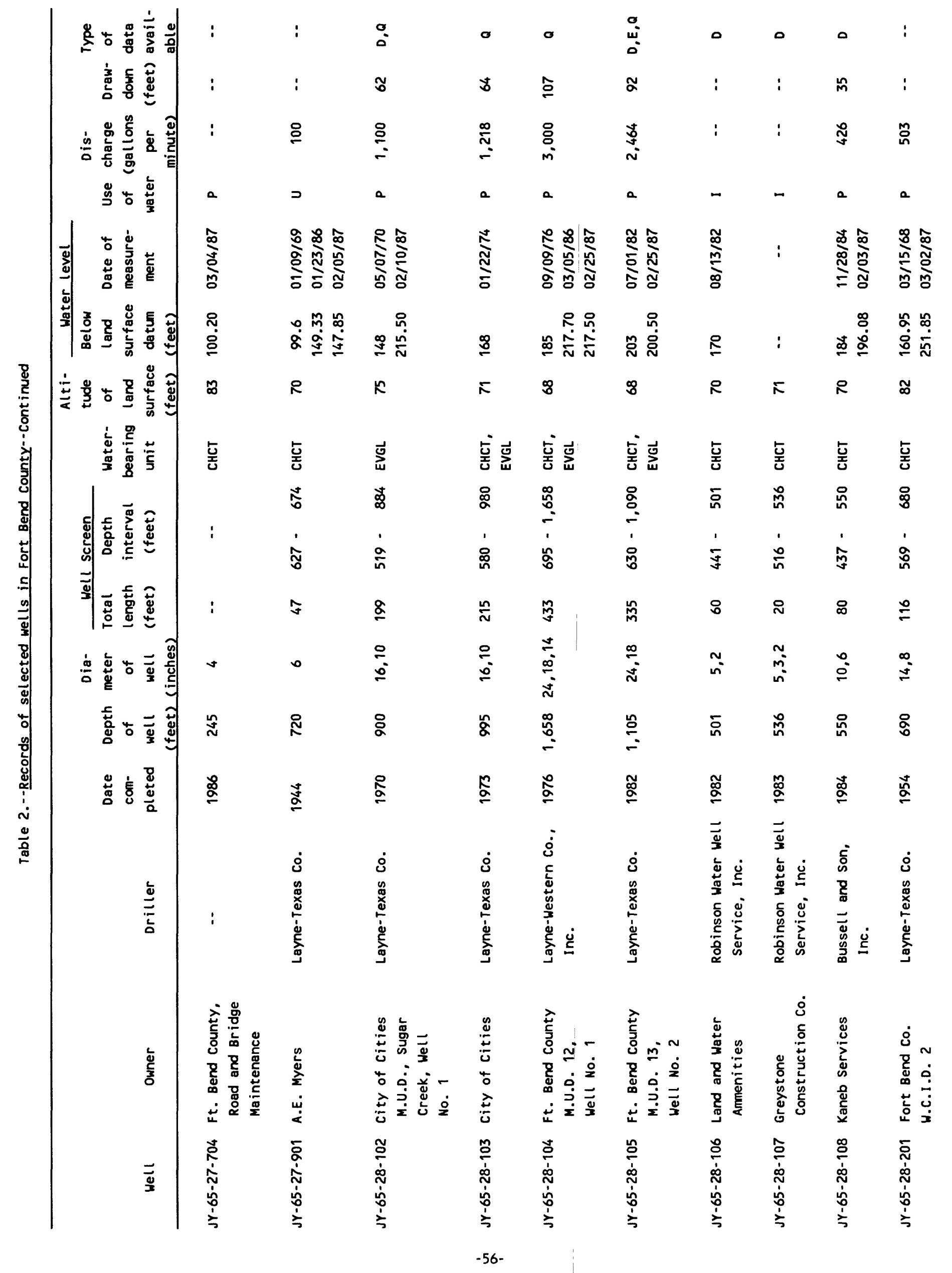




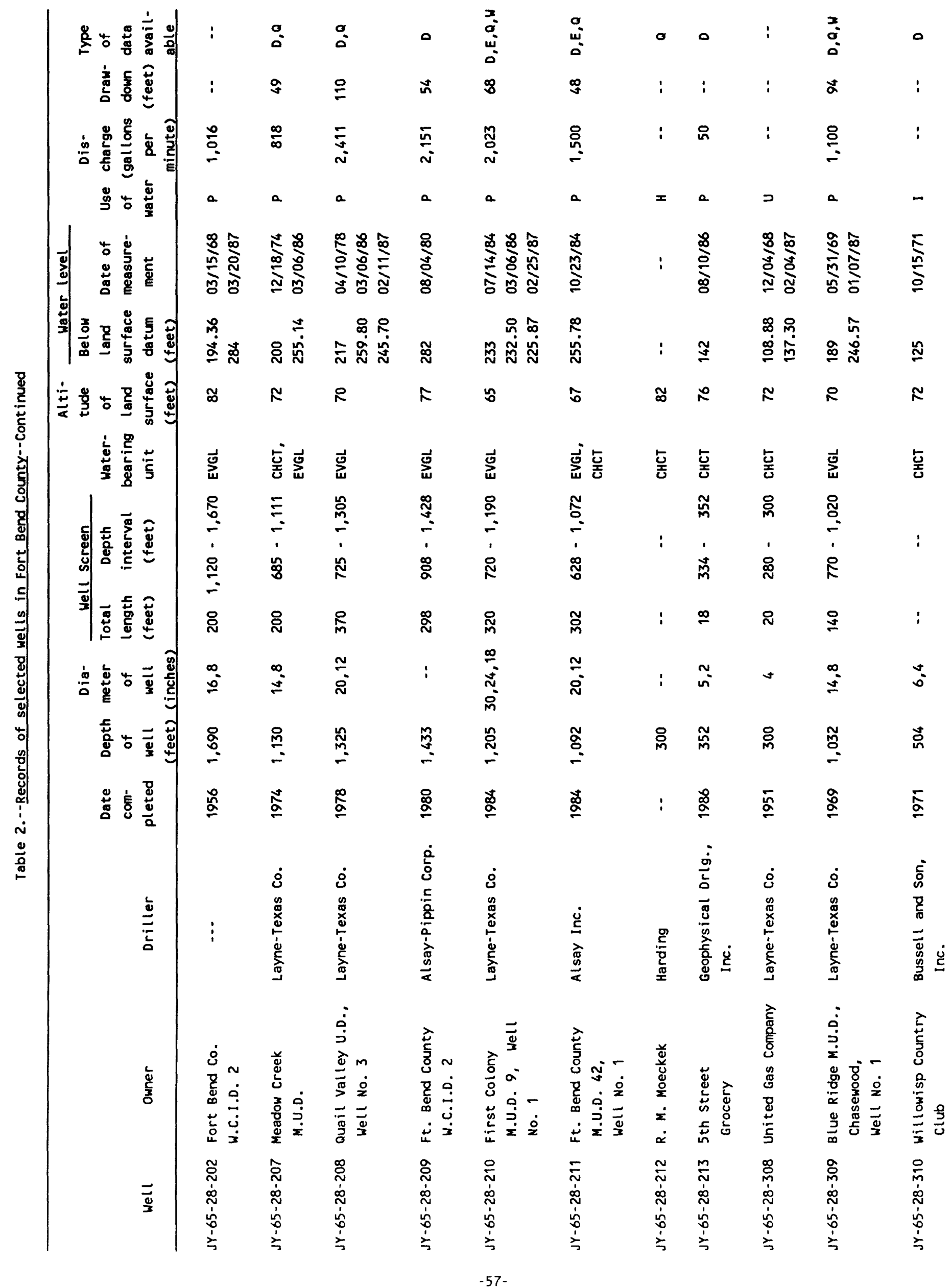




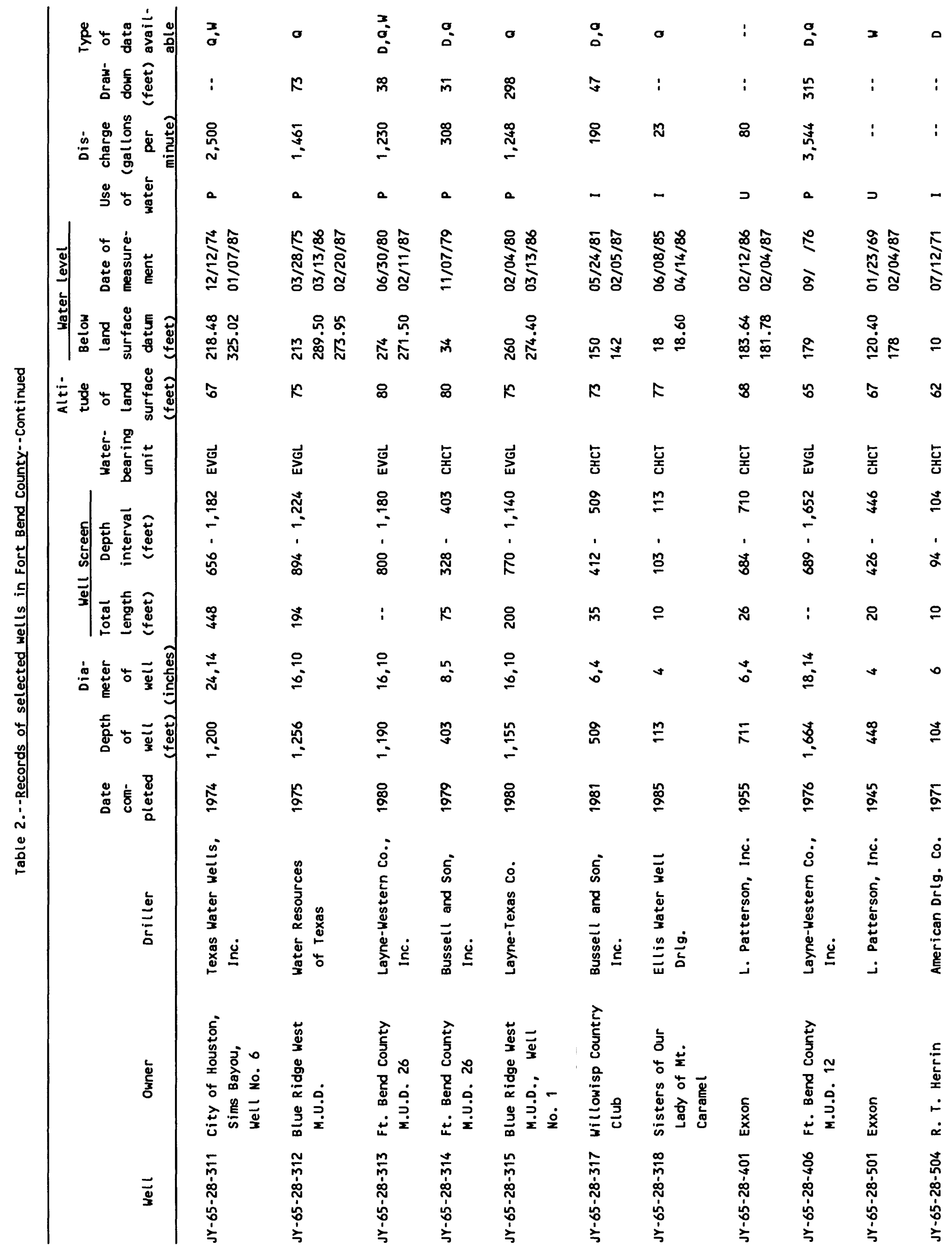




\begin{tabular}{|c|c|c|c|c|c|c|c|c|}
\hline 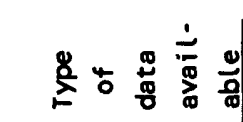 & 。 & $\stackrel{8}{\circ}: \frac{3}{0}$ & $\stackrel{\leftrightarrow}{\ddot{\leftrightarrow}}$ & 品 & $\sigma$ & $\stackrel{8}{\circ}$ & & \\
\hline 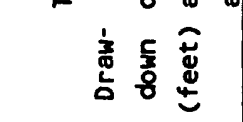 & : & $8 \mathrm{~g}$ & $\because$ & $:$ & & $=$ & & $=$ \\
\hline 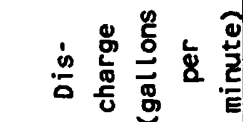 & $\stackrel{8}{:}$ & $\$$ & 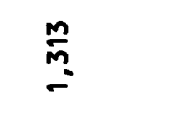 & $\stackrel{g}{:}$ & & 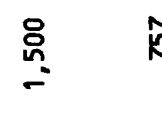 & 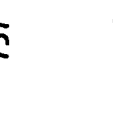 & \\
\hline$\frac{5}{3}$ & $\therefore$ & & & & - & - & & \\
\hline 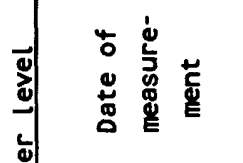 & 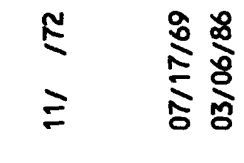 & 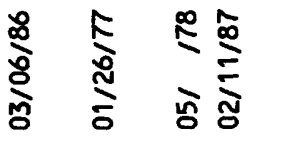 & 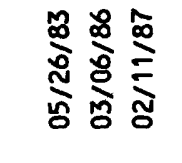 & 善 & 戀 & 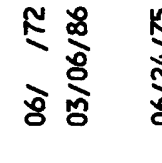 & & 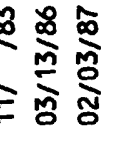 \\
\hline 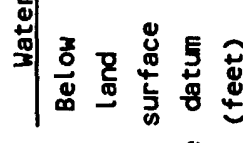 & 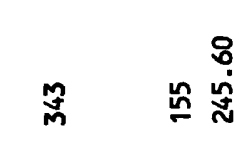 & 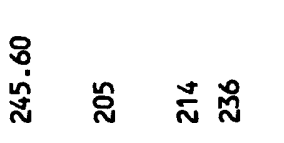 & 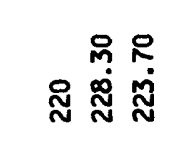 & & 8 & 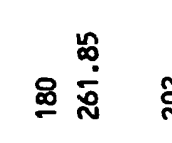 & & 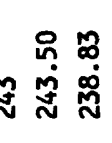 \\
\hline 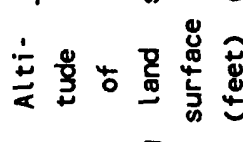 & $8 \quad 8$ & $8 z$ & $\therefore$ & 8 & ' & ${ }^{k}$ & $=$ & $=$ \\
\hline 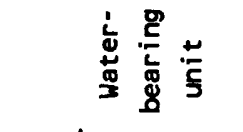 & 总总喜 & 豆畐 & 总 & 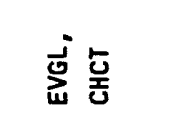 & $\frac{5}{5}$ & 喜 & 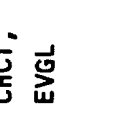 & \\
\hline 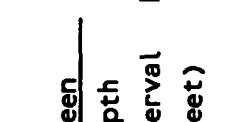 & $\stackrel{g}{g}$ & 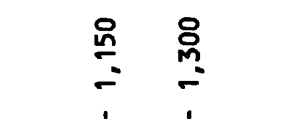 & 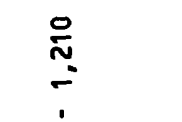 & 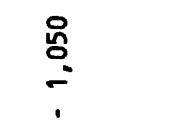 & & 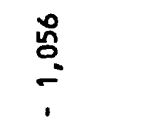 & & 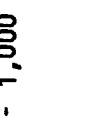 \\
\hline 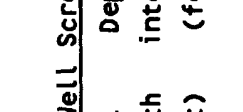 & $\stackrel{\check{z}}{3}$ & $\dot{\grave{s}}$ & $\frac{n}{\hat{x}}$ & 8 & & 8 & 8 & 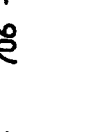 \\
\hline 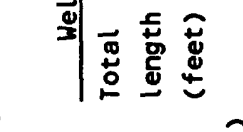 & $\cong \cong$ & $\cong$ & 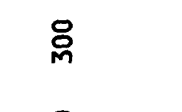 & ฐ & : & $\approx$ & 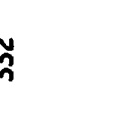 & \\
\hline 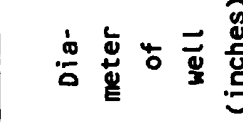 & $=\dddot{\Xi}$ & $\ddot{\Xi}$ & $\frac{\mathscr{g}}{\dot{B}}$ & ؛̊: & 。 & $\simeq$ & & 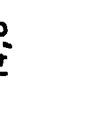 \\
\hline 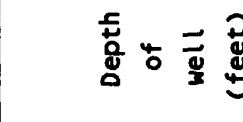 & ฐู & $\cong$ & § & $\stackrel{\dddot{a}}{:}$ & 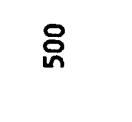 & $\stackrel{5}{5}$ & & 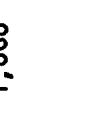 \\
\hline 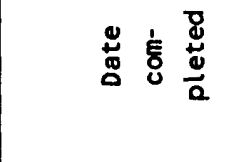 & 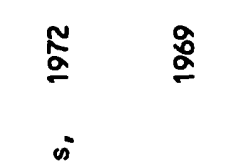 & ڤ̊ㅗㅇ & $\$$ & $\stackrel{g}{g}$ & $\stackrel{x}{g}$ & 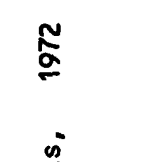 & & \\
\hline & & & & & & & & \\
\hline & 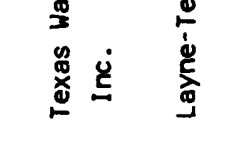 & & & & & & & \\
\hline & & & & 拿高 & & & & \\
\hline & 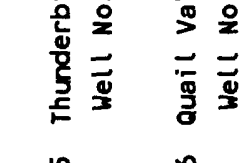 & & 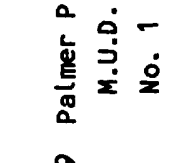 & 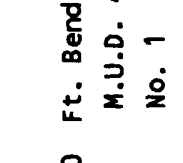 & $\frac{a}{2}$ & 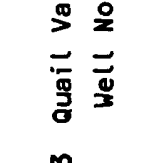 & & 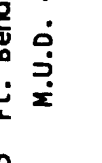 \\
\hline & 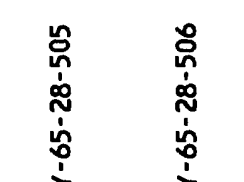 & 意耪 & & & & & & \\
\hline
\end{tabular}




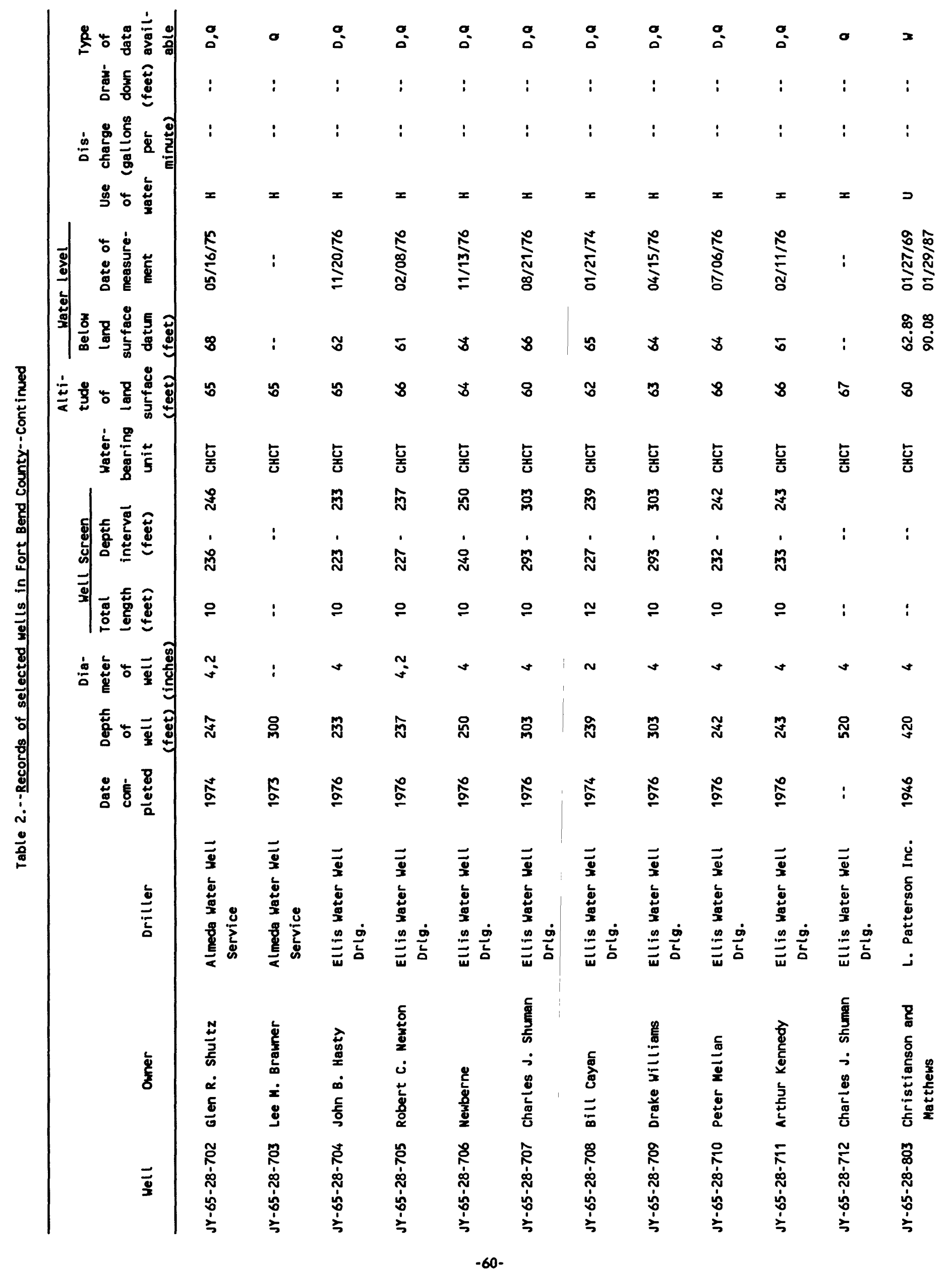




\begin{tabular}{|c|c|c|c|c|c|c|c|c|c|c|c|c|}
\hline 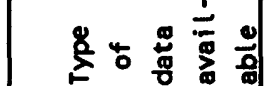 & 0 & $:$ & 0 & 0 & 0 & $=$ & $\frac{2}{0}$ & 0 & $\stackrel{0}{0}$ & 0 & $\stackrel{8}{\circ}$ & 0 \\
\hline 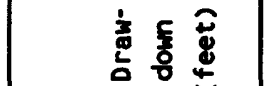 & : & : & : & : & : & $:$ & : & $n$ & : & : & ఉ & : \\
\hline 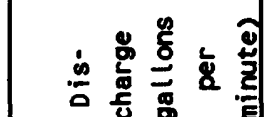 & $:$ & $:$ & : & $\stackrel{8}{0}$ & $\stackrel{i}{0}$ & 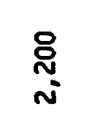 & 尺ి & 各 & $\underset{\substack{0 \\
\text { N }}}{ }$ & $:$ & $\stackrel{\circ}{!}$ & 品 \\
\hline 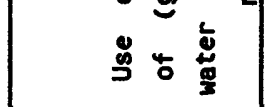 & $=$ & $\infty$ & - & - & $x$ & D & $a$ & $a$ & a & $x$ & $a$ & $=$ \\
\hline 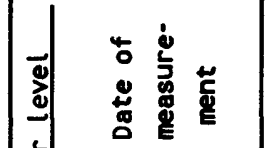 & $\stackrel{2}{\stackrel{2}{2}}$ & 商 & 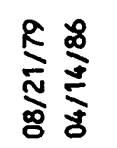 & 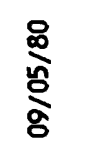 & 荵 & $\stackrel{2}{\stackrel{2}{2}}$ & 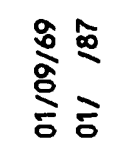 & 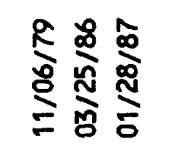 & 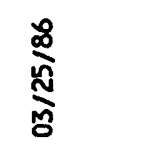 & : & 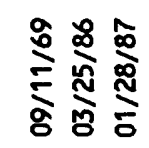 & $\begin{array}{l}\hat{s} \\
\equiv\end{array}$ \\
\hline 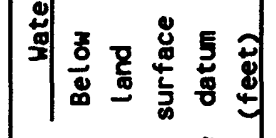 & 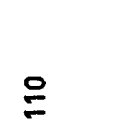 & $\underset{\mathscr{n}}{\mathscr{m}}$ & $\stackrel{\hat{y}}{0}$ & $\infty$ & : & 蕰 & 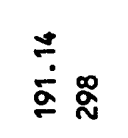 & 总离 & 品 & $:$ & $=\stackrel{0}{i}$ & $\stackrel{\infty}{\sim}$ \\
\hline 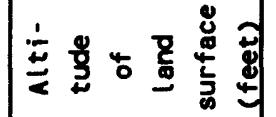 & 8 & in & 8 & $\stackrel{\circ}{\circ}$ & $\overline{0}$ & 8 & ถ & 8 & 8 & 8 & ถ & $\Sigma$ \\
\hline 悹 & 㲋 & 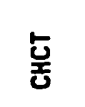 & $\stackrel{t}{i}$ & 浐 & 喜 & 喜 & 喜 & 喜 & 喜 & $\stackrel{t}{\underline{t}}$ & 喜 & $\underline{\underline{\underline{u}}}$ \\
\hline & 8 & & $\approx$ & $\triangleright$ & 品 & ○ & 怘 & 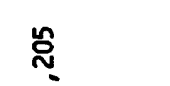 & 巳্̣ & & 葛 & $\stackrel{\$}{*}$ \\
\hline 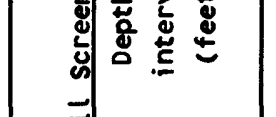 & 品 & $:$ & $\dot{\leftrightarrow}$ & 8 & $\stackrel{\text { Nิ }}{\text { }}$ & i & $\hat{\kappa}$ & R & : & $:$ & 8 & 8 \\
\hline 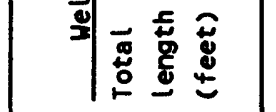 & i & $:$ & $\therefore$ & $\div$ & $\div$ & 号 & 욤 & $\stackrel{\circ}{n}$ & $\stackrel{8}{3}$ & $:$ & 9 & \& \\
\hline 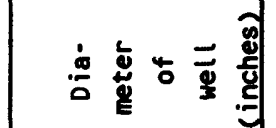 & $\mathfrak{z}$ & + & $\circ$ & ○ & $\bullet$ & $\stackrel{\sim}{\infty}$ & $:$ & $\stackrel{\sim}{\infty}$ & $\cong$ & $\sim$ & $\stackrel{\infty}{=}$ & $\circ$ \\
\hline 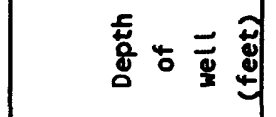 & 8 & ฉ̊ & $\approx$ & ○ & 忿 & $\approx$ & $\frac{\circ}{a}$ & $\underset{\mathrm{N}}{\stackrel{乛}{\sim}}$ & $\stackrel{\mathbb{\widetilde { N }}}{=}$ & $\stackrel{\circ}{ }$ & $\stackrel{8}{:}$ & $\mathbb{q}$ \\
\hline 岁 & $\stackrel{2}{g}$ & $:$ & 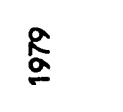 & $\stackrel{\circ}{\mathscr{g}}$ & $\stackrel{2}{g}$ & $\stackrel{q}{a}$ & $\stackrel{\circ}{\circ}$ & $\stackrel{?}{g}$ & 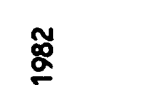 & $\stackrel{8}{2}$ & $\stackrel{\circ}{g}$ & $\stackrel{\infty}{\alpha}$ \\
\hline$\stackrel{\varrho}{=}$ & 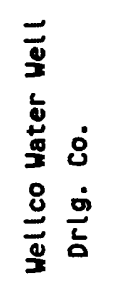 & 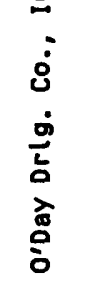 & 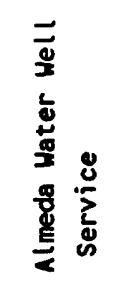 & 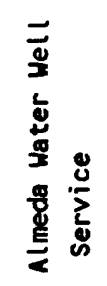 & 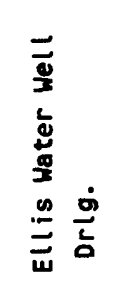 & 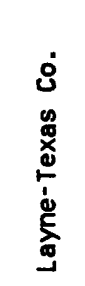 & 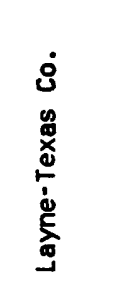 & 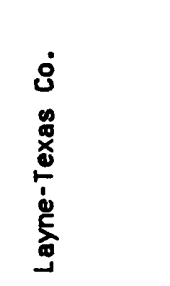 & 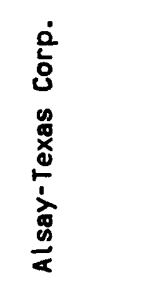 & $:$ & 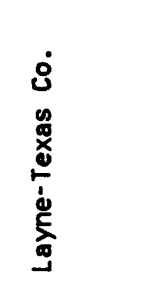 & 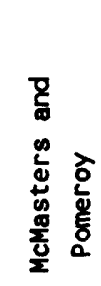 \\
\hline 产 & 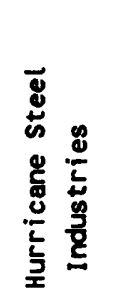 & 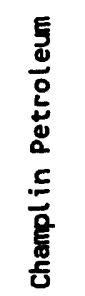 & 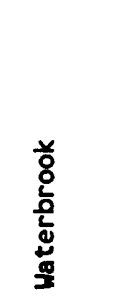 & 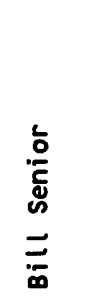 & $\begin{array}{l}\frac{\sigma}{\tilde{g}} \\
\frac{5}{5} \\
\dot{\omega} \\
\dot{L} \\
\dot{0}\end{array}$ & 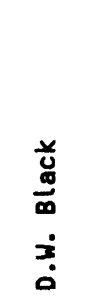 & 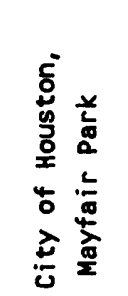 & 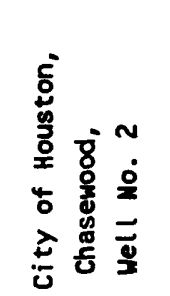 & 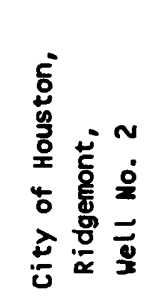 & 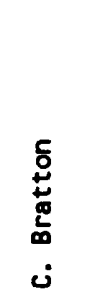 & 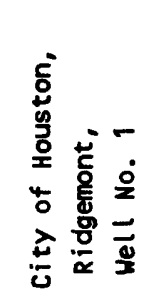 & 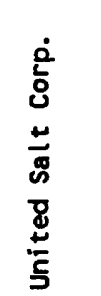 \\
\hline$\overline{\overline{\underline{I}}}$ & 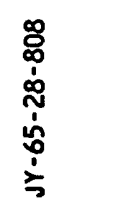 & 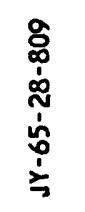 & 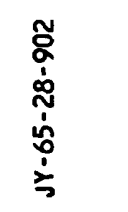 & 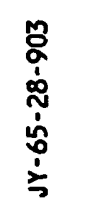 & 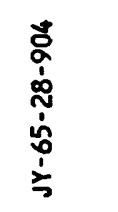 & $\begin{array}{l}\bar{o} \\
\grave{d} \\
\grave{\omega} \\
\grave{L} \\
\grave{j}\end{array}$ & 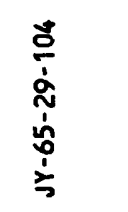 & 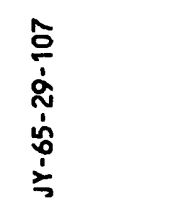 & 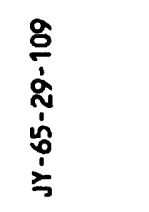 & 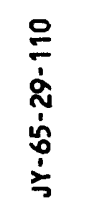 & 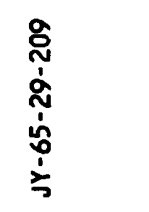 & 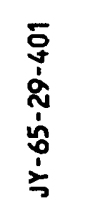 \\
\hline
\end{tabular}




\begin{tabular}{|c|c|c|c|c|c|c|c|c|c|c|c|}
\hline 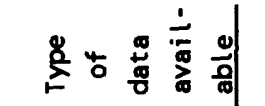 & : & 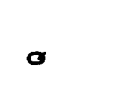 & 0 & $\because$ & 0 & 0 & $x$ & $:$ & 苛 & 0 & $\stackrel{a}{\circ}$ \\
\hline & $:$ & $:$ & $:$ & $:$ & : & $:$ & $:$ & $:$ & in & $:$ & $:$ \\
\hline 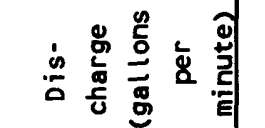 & $\stackrel{n}{0}$ & $:$ & $:$ & $:$ & $:$ & 品 & $:$ & $:$ & $\stackrel{\tilde{0}}{=}$ & 品 & 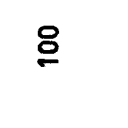 \\
\hline 0 & $=$ & - & $z$ & $=$ & $z$ & $=$ & $\mathrm{J}$ & $x$ & a & - & $=$ \\
\hline 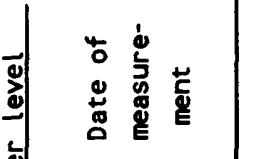 & 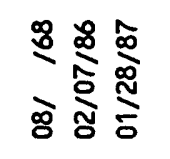 & 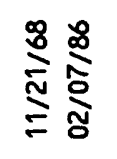 & $\frac{0}{\circ}$ & 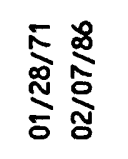 & 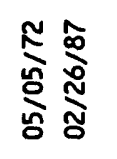 & 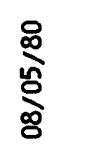 & 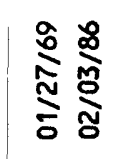 & 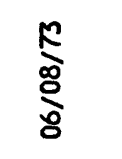 & 总商 & 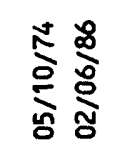 & $\stackrel{\bar{\Phi}}{\stackrel{N}{\Xi}}$ \\
\hline & 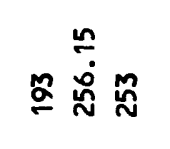 & 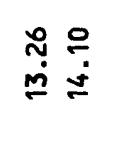 & 喜 & $\stackrel{\infty}{\simeq} \pm$ & 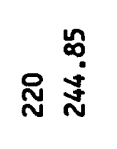 & 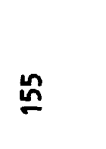 & 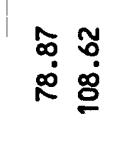 & $\approx$ & 蛋 & $\pm \stackrel{\mathscr{Q}}{\dot{0}}$ & $\simeq$ \\
\hline 30 & $\approx$ & 8 & $\tilde{\circ}$ & 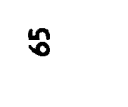 & 8 & $\tilde{\circ}$ & $\hat{\theta}$ & $R$ & $\approx$ & $R$ & R \\
\hline 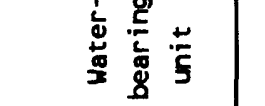 & $\stackrel{\underline{\underline{t}}}{\underline{t}}$ & $\stackrel{\underline{t}}{\underline{t}}$ & 橲 & $\stackrel{\underline{s}}{\tilde{t}}$ & 喜 & 壱 & $\stackrel{t}{s}$ & $\stackrel{t}{\underline{s}}$ & 喜 & 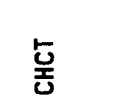 & 预 \\
\hline & $n$ & 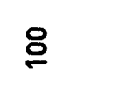 & $\stackrel{n}{\alpha}$ & 飠 & 禺 & 品 & & 8 & 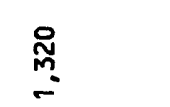 & $\underline{E}$ & $\kappa$ \\
\hline & $\stackrel{\infty}{i n}$ & 2 & in & 品 & $\bar{\Phi}$ & 售 & 1 & $\bar{J}$ & $\dot{ळ}$ & $\Xi$ & $\ddot{\circ}$ \\
\hline $\begin{array}{l}0 \\
0\end{array}$ & in & 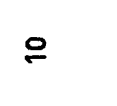 & 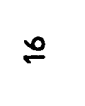 & $\stackrel{i}{2}$ & $\cong$ & $\stackrel{\sim}{\sim}$ & $:$ & $\stackrel{2}{2}$ & 总 & i & i \\
\hline 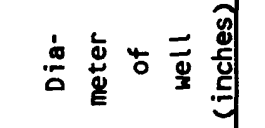 & $\infty$ & $\checkmark$ & $\tilde{v}$ & : & $\stackrel{*}{\circ}$ & $\stackrel{4}{0}$ & $\checkmark$ & $\tilde{s}$ & $\stackrel{5}{i}$ & $\stackrel{+}{\infty}$ & $\stackrel{\circ}{\infty}$ \\
\hline 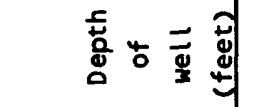 & 莒 & $\stackrel{8}{ }$ & $\stackrel{\infty}{0}$ & 兽 & 웅 & 鬲 & $\ddot{g}$ & 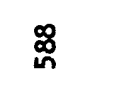 & 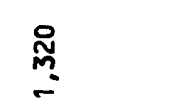 & $\underline{R}$ & $k$ \\
\hline 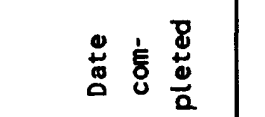 & $\frac{8}{g}$ & 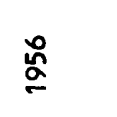 & $\stackrel{\circ}{\alpha}$ & $\underline{\underline{\alpha}}$ & $\stackrel{N}{\alpha}$ & $\stackrel{\circ}{\mathscr{g}}$ & 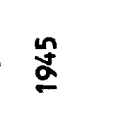 & $\stackrel{\text { so }}{\alpha}$ & $\stackrel{\alpha}{\alpha}$ & $\underline{\underline{z}}$ & $\stackrel{\bar{\alpha}}{\stackrel{\alpha}{ }}$ \\
\hline & 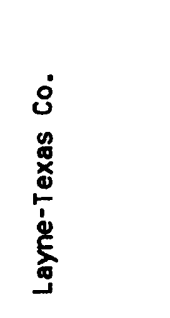 & : & 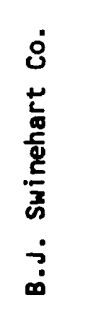 & 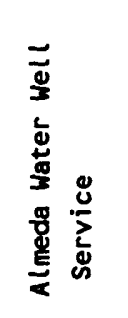 & 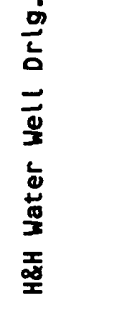 & 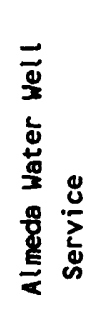 & 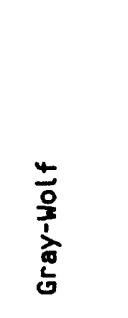 & 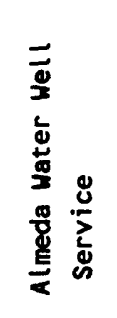 & 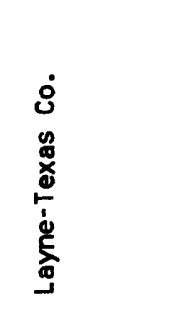 & 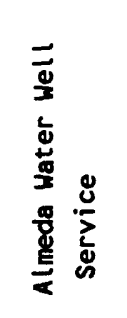 & 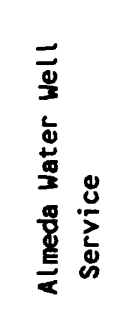 \\
\hline
\end{tabular}

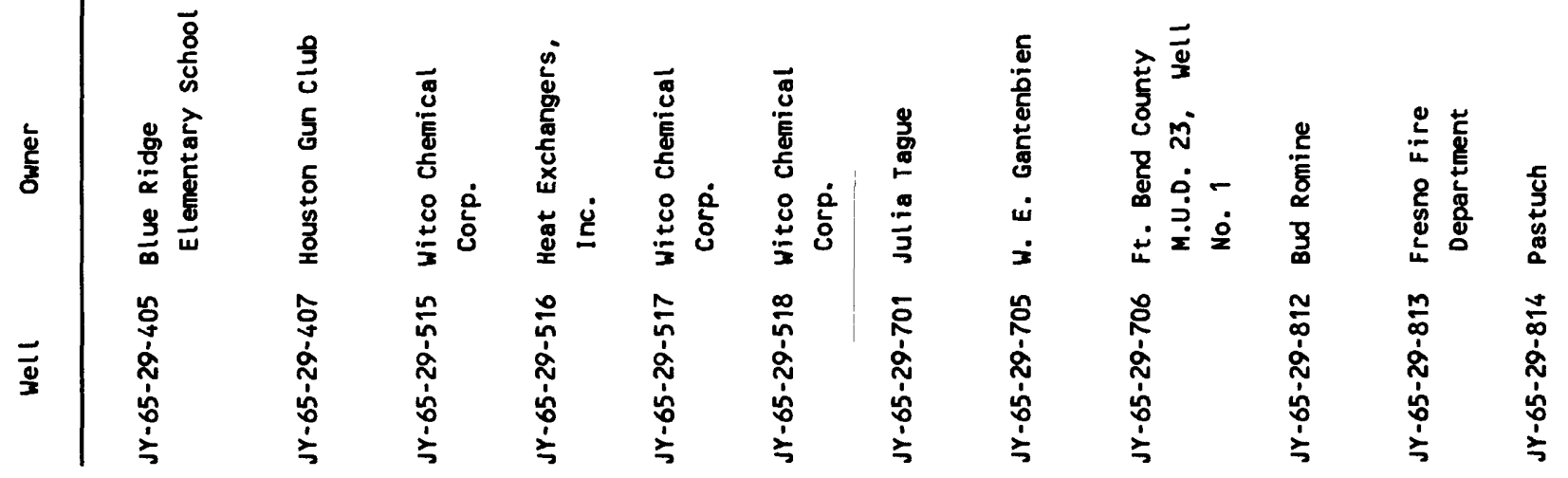




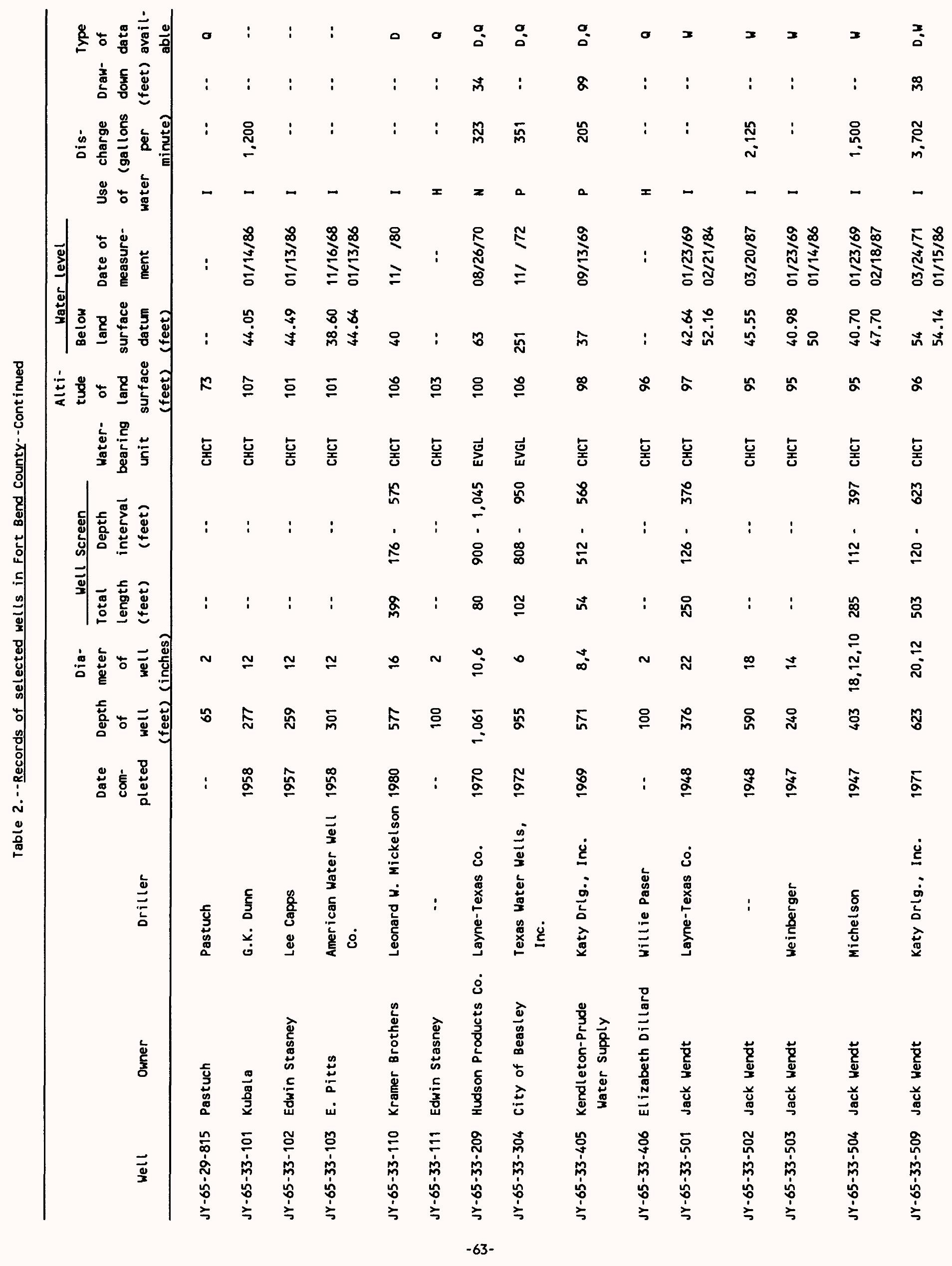




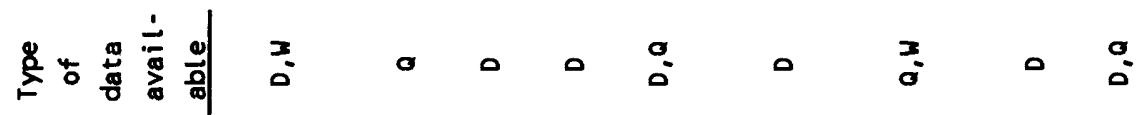

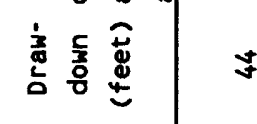

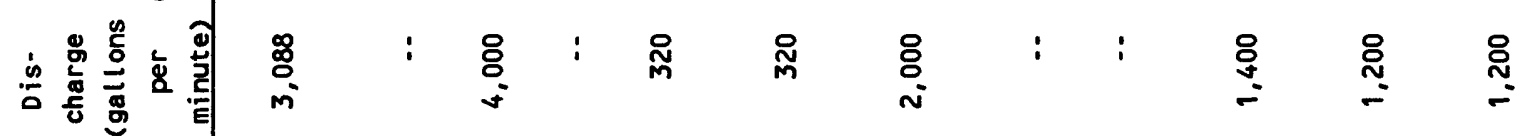

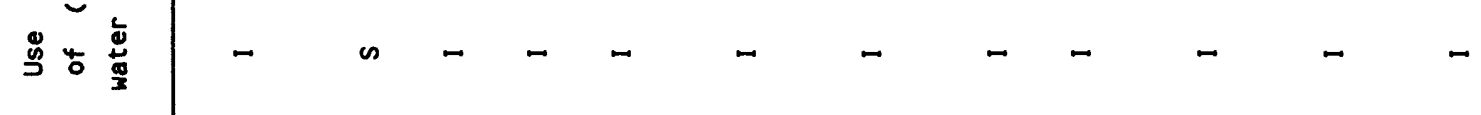

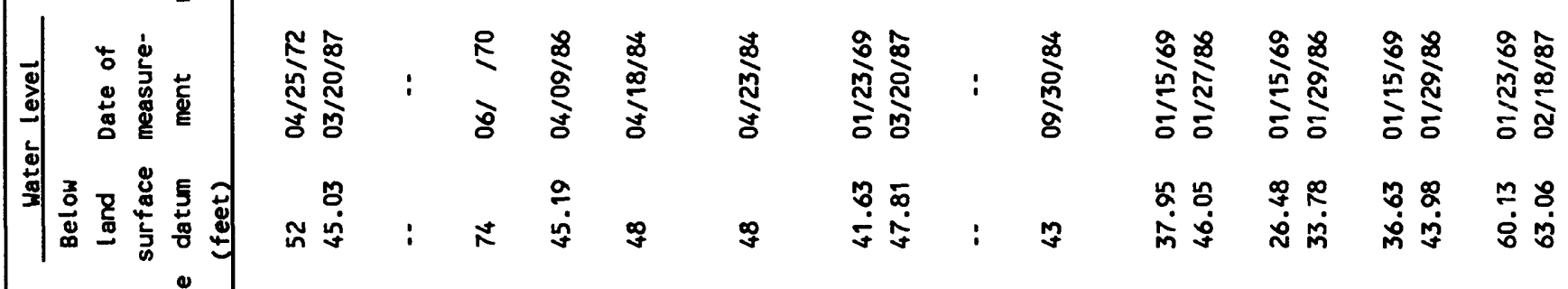

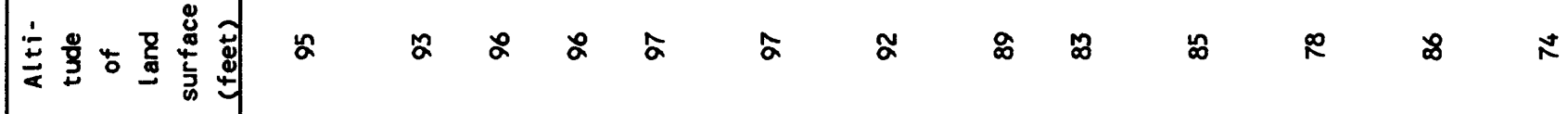

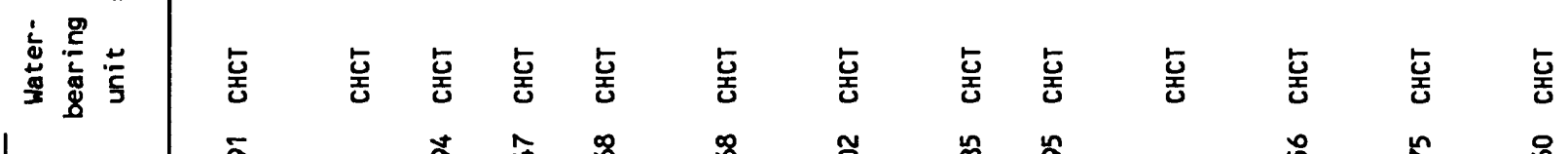

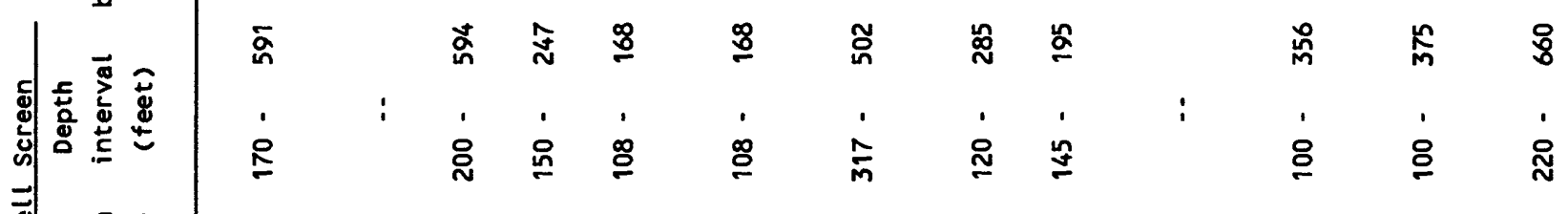
辛昰䓌密

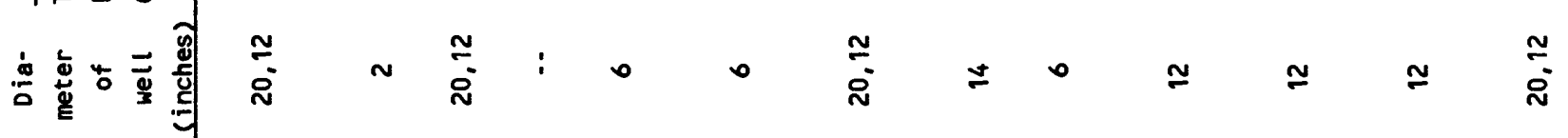

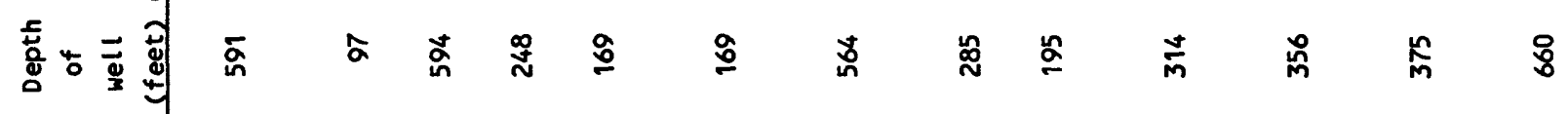

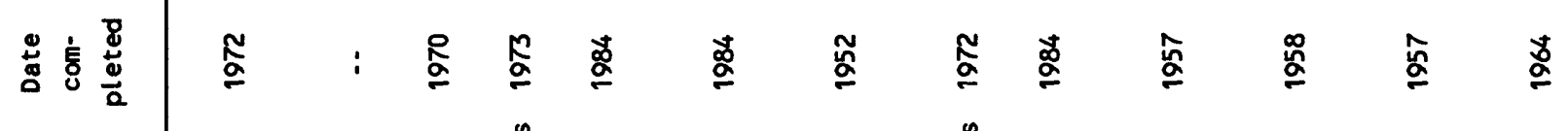

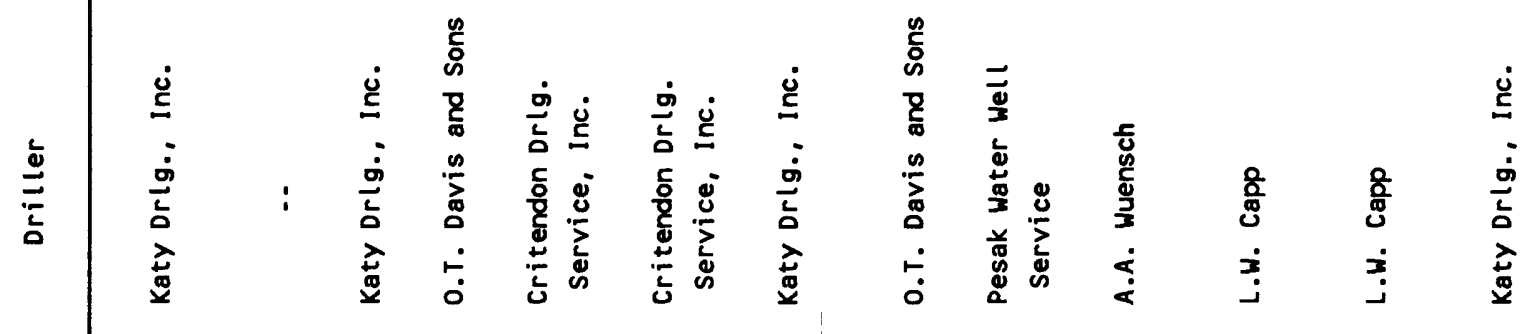

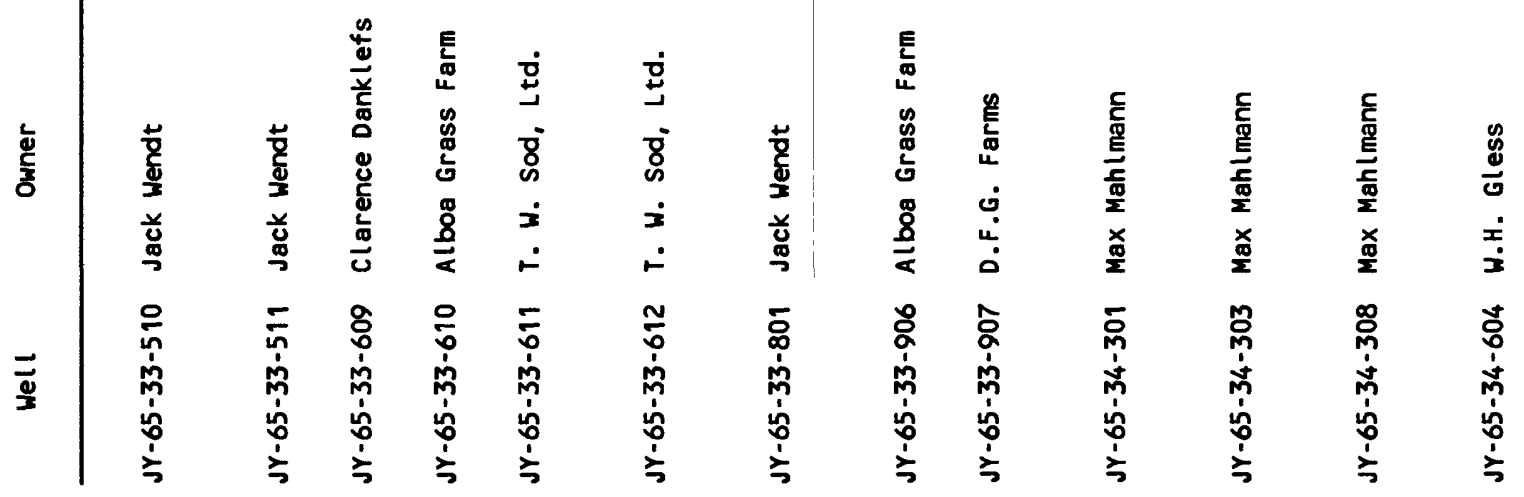




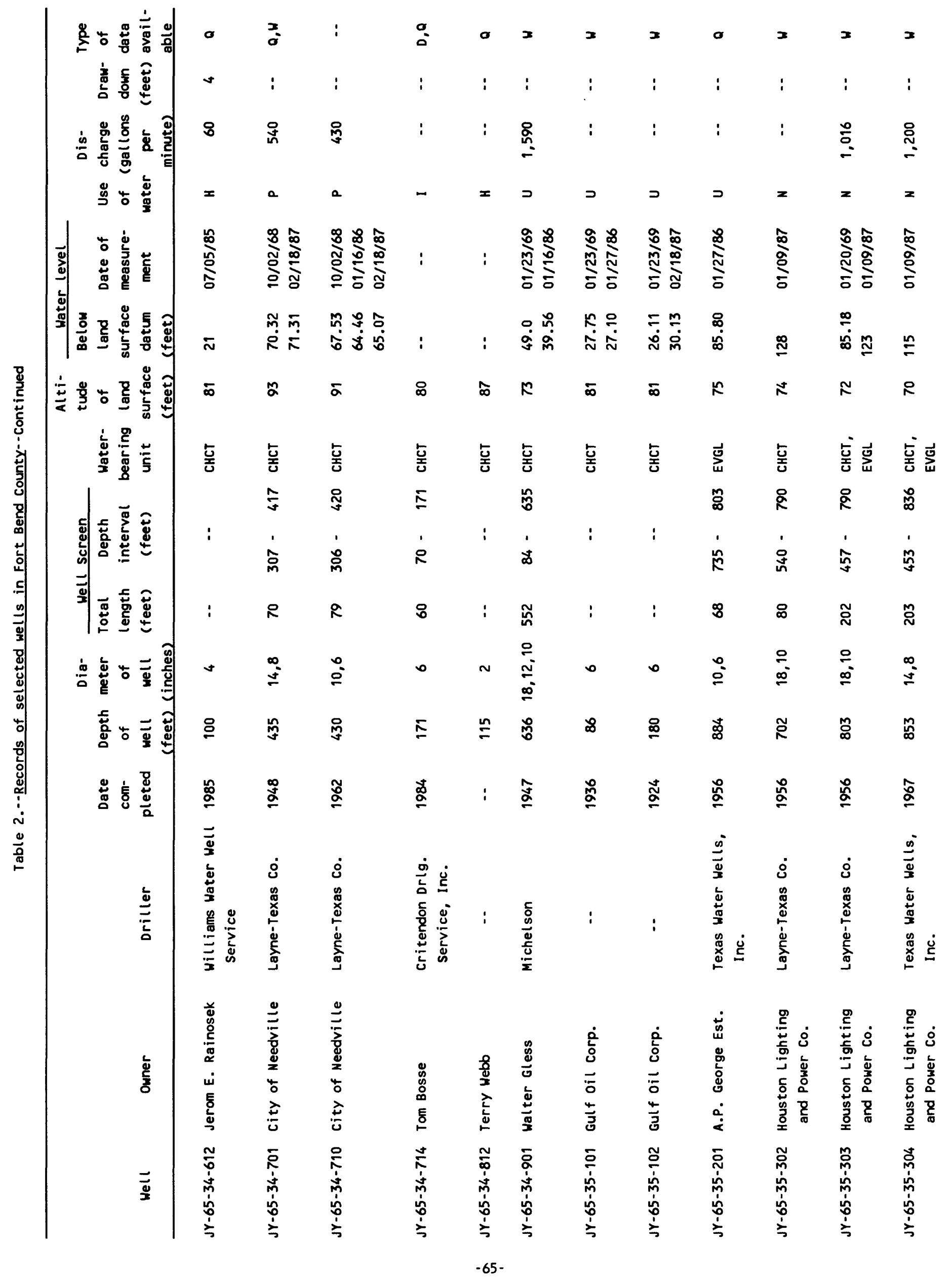




\begin{tabular}{|c|c|c|c|c|c|c|c|c|c|c|}
\hline 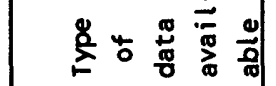 & $\stackrel{a}{\circ}$ & $\stackrel{g}{\circ}$ & 0 & : & : & : & : & : & : & : \\
\hline 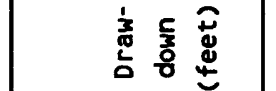 & i & $\bar{J}$ & q & : & : & : & : & : & $:$ & : \\
\hline 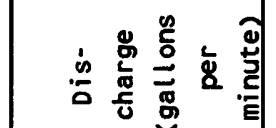 & 品 & 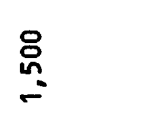 & $\stackrel{\substack{a \\
i}}{i}$ & $:$ & $\stackrel{\circ}{\stackrel{\leftrightarrow}{\circ}}$ & $:$ & $\approx$ & $\bar{\alpha}$ & $\stackrel{\widetilde{\Xi}}{\check{\sigma}}$ & 品 \\
\hline 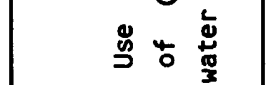 & $=$ & $=$ & $=$ & $=$ & $=$ & $=$ & $=$ & د & $=$ & $=$ \\
\hline 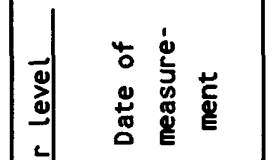 & 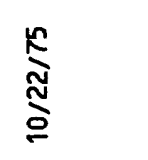 & $\frac{\overline{0}}{\stackrel{5}{5}}$ & 帘 & 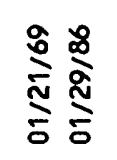 & 离 & 总 & 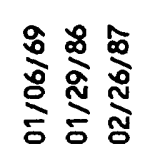 & 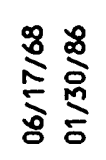 & 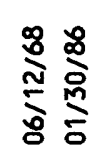 & 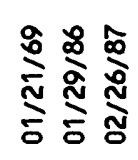 \\
\hline 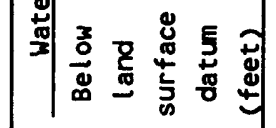 & 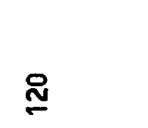 & $\stackrel{̊}{\cong}$ & $\stackrel{n}{=}$ & 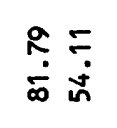 & $\stackrel{?}{\dot{i}}$ & 要 & 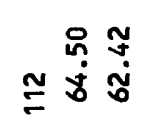 & $\stackrel{\mathscr{D}}{\stackrel{\mathscr{m}}{=}}$ & $\stackrel{n}{a}$ & 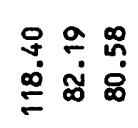 \\
\hline 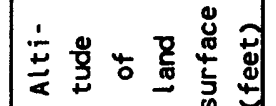 & $R$ & $\kappa$ & $R$ & 8 & $\hat{\imath}$ & 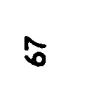 & $\hat{\sigma}$ & 尺 & $\infty$ & 8 \\
\hline 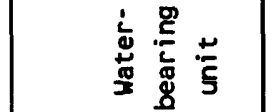 & 鯜 & $\overrightarrow{\underline{s}}$ & 空 & 蹇 & 厗 & 产 & 厗 & 喜 & $\underline{\underline{T}}$ & $\stackrel{\underline{\underline{z}}}{\underline{t}}$ \\
\hline & 触 & 笛 & \$ & & $\underset{R}{n}$ & 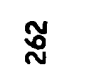 & $\$$ & do & 总 & $\hat{g}$ \\
\hline 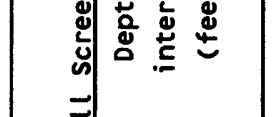 & 8 & \&্̧ & g & : & 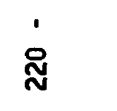 & $\hat{\approx}$ & $\hat{n}$ & 声 & $\stackrel{D}{N}$ & 字 \\
\hline 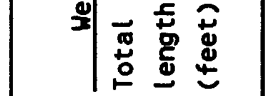 & 只 & $\stackrel{\leftrightarrow}{n}$ & $\underset{N}{\infty}$ & $:$ & $:$ & $\cong$ & 8 & 8 & 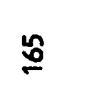 & 2 \\
\hline 它兽 & 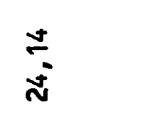 & 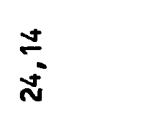 & $\stackrel{ \pm}{\sim}$ & $\stackrel{\circ}{\cong}$ & $\frac{9}{i}$ & $\simeq$ & $\stackrel{\circ}{i}$ & $\stackrel{\circ}{i}$ & $\stackrel{\circ}{i}$ & 足 \\
\hline 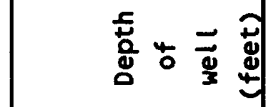 & $\bar{\Xi}$ & 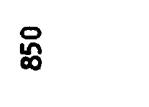 & $\stackrel{\circ}{\otimes}$ & $\stackrel{\leftrightarrow}{\stackrel{\leftrightarrow}{*}}$ & $\stackrel{2}{N}$ & 呙 & $\bar{g}$ & 总 & 吕 & $\hat{g}$ \\
\hline 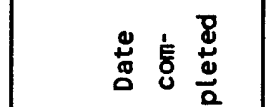 & $\stackrel{\kappa}{\alpha}$ & go & $\stackrel{\bar{\Phi}}{\stackrel{D}{ }}$ & 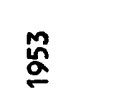 & $\underline{\underline{s}}$ & gू & $\stackrel{\mathrm{g}}{\mathrm{a}}$ & $\stackrel{8}{8}$ & $\stackrel{\circ}{\circ}$ & $\stackrel{\Phi}{\varrho}$ \\
\hline 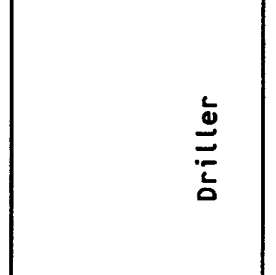 & 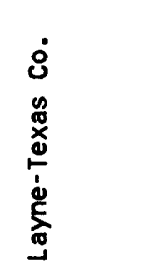 & 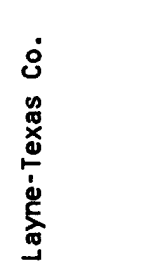 & 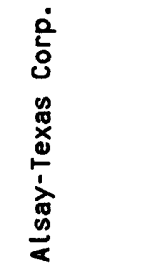 & 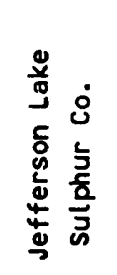 & 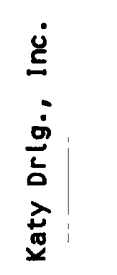 & 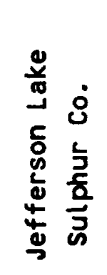 & 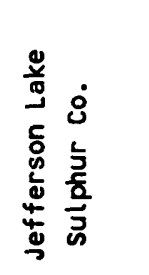 & $\begin{array}{l}\dot{\dot{g}} \\
\dot{\Phi} \\
\dot{\Delta} \\
\dot{\Delta} \\
\dot{\vec{v}}\end{array}$ & 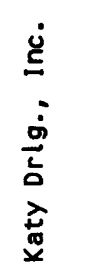 & 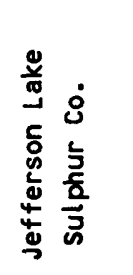 \\
\hline 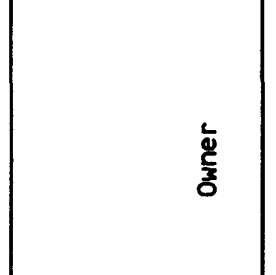 & 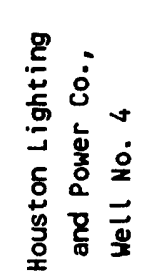 & 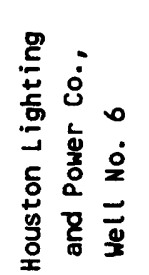 & 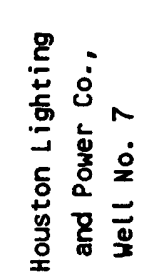 & 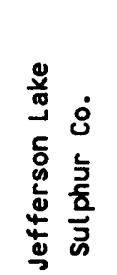 & 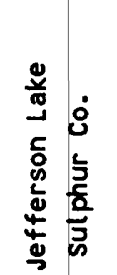 & 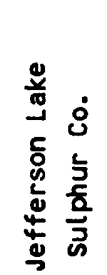 & 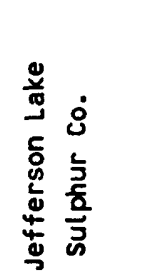 & 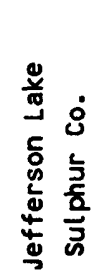 & 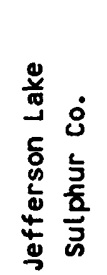 & 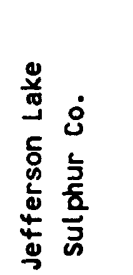 \\
\hline$\overline{\underline{\underline{a}}}$ & 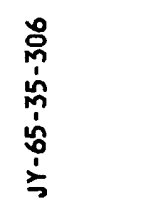 & 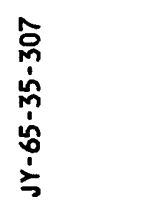 & 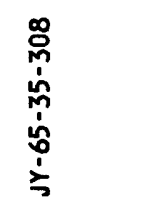 & $\begin{array}{l}\bar{p} \\
\dot{\omega} \\
\dot{\omega} \\
\dot{0} \\
\grave{1} \\
\grave{3}\end{array}$ & 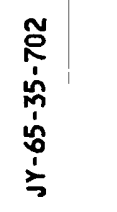 & 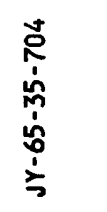 & 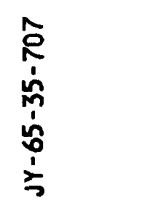 & 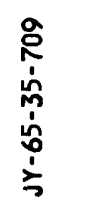 & 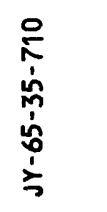 & 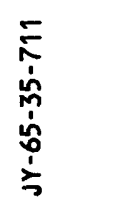 \\
\hline
\end{tabular}




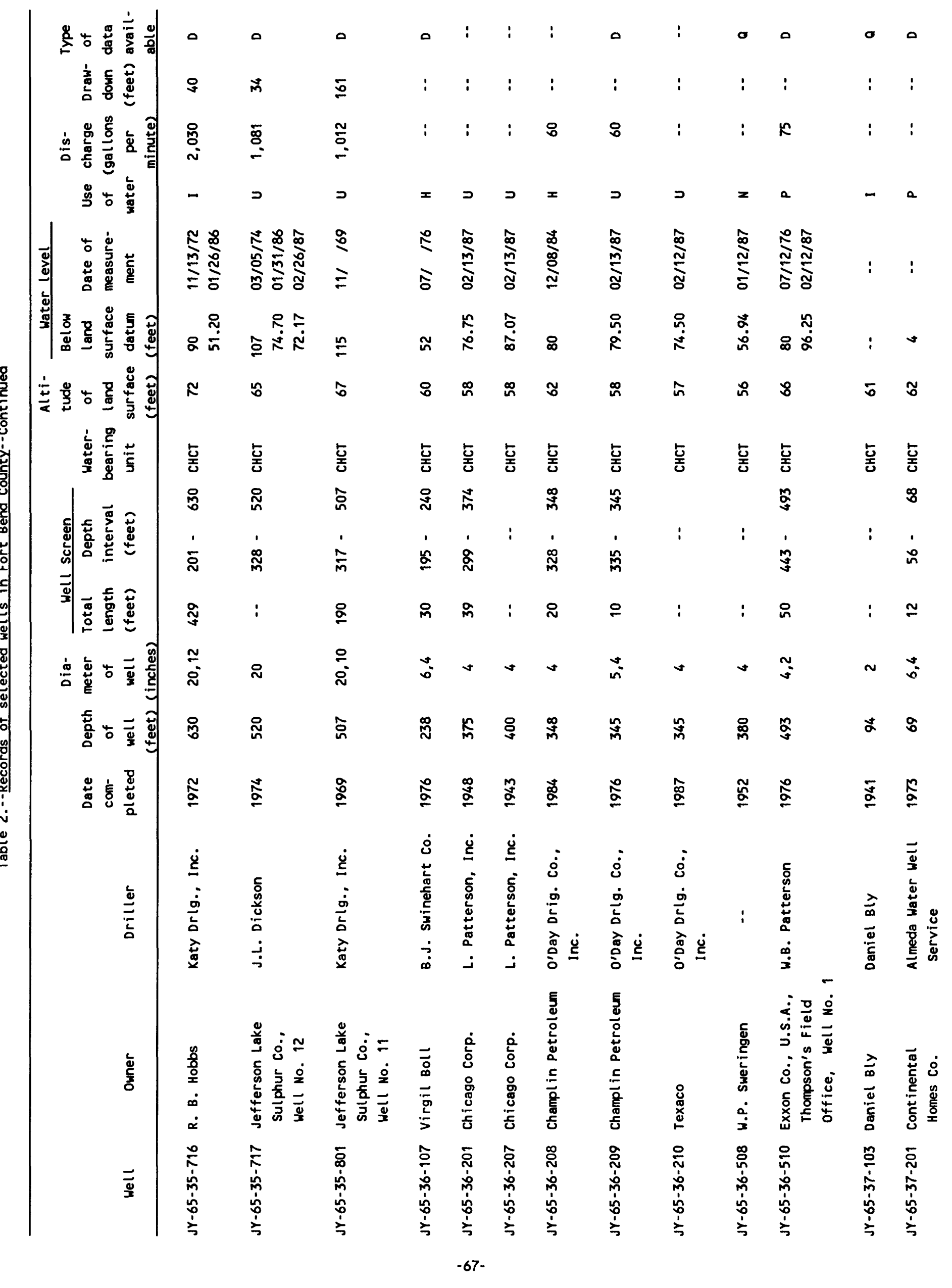




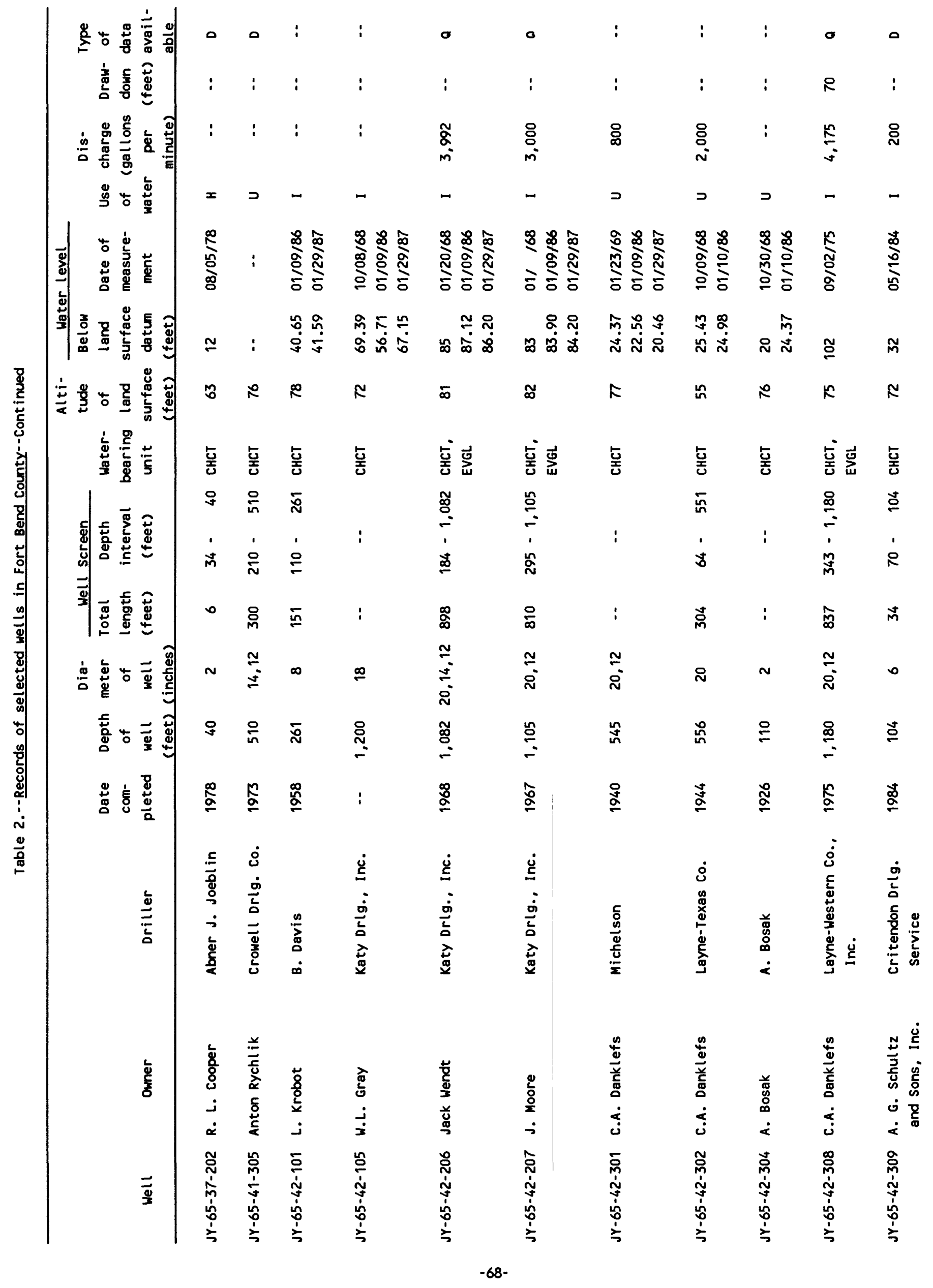




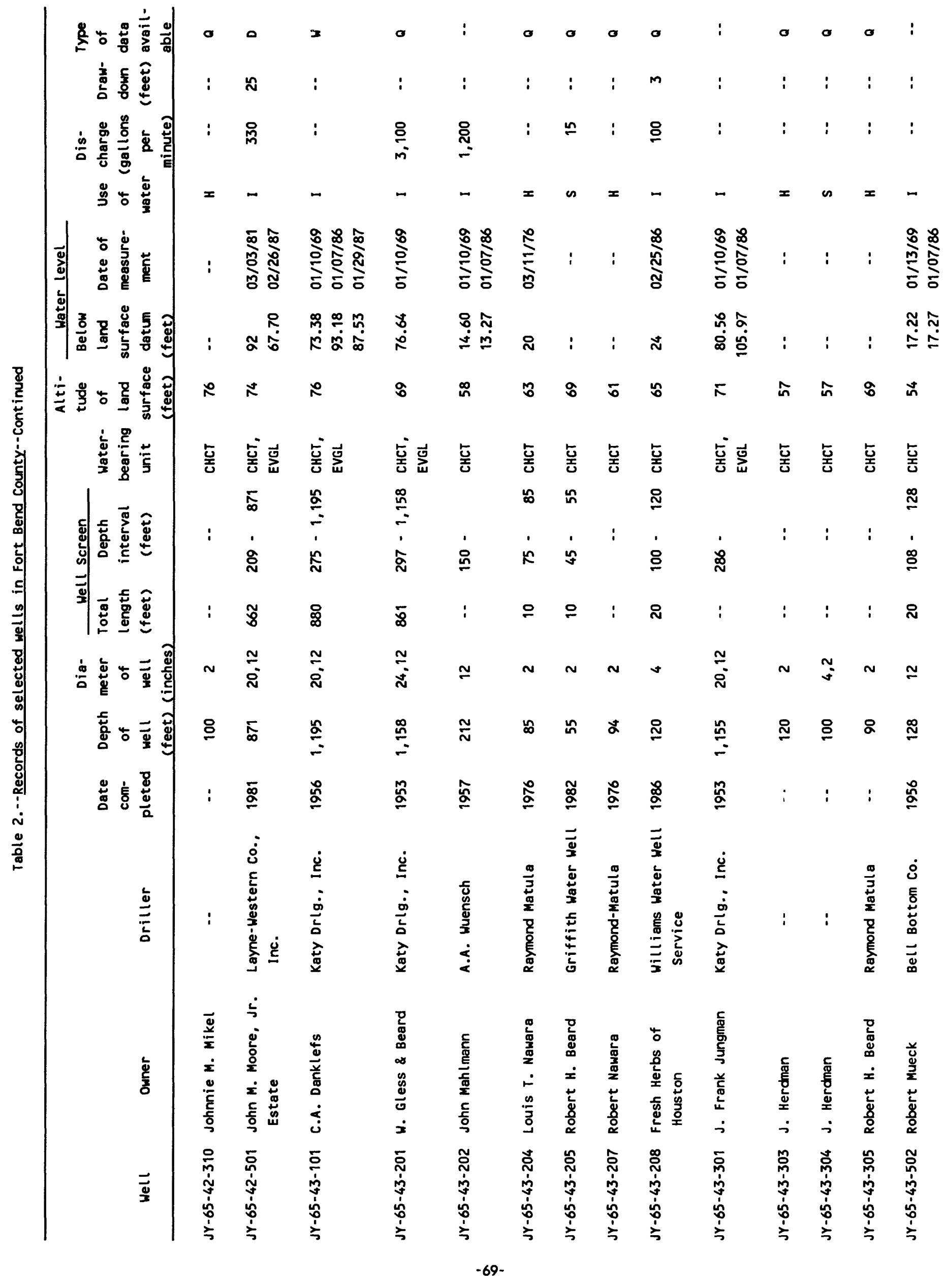




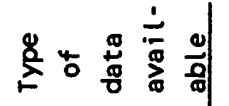

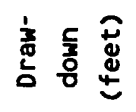

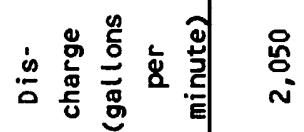

$\underset{\substack{\text { i } \\ \text { N }}}{(}$

8 : ํㅗㅇ

$\stackrel{\mathscr{m}}{3} \div$

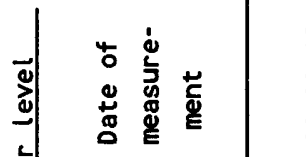

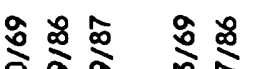

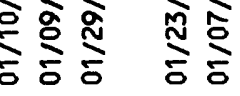

突

商

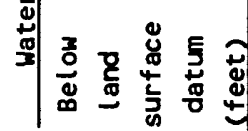

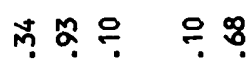

下 $\approx$

ळ

๓⿴囗十

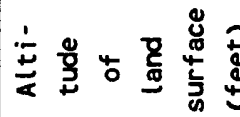
这高 $\frac{5}{5}$ ถ̊

in in in in in

กิ $\equiv \stackrel{m}{=}$

$\stackrel{\cong}{\cong} \stackrel{\infty}{=}$

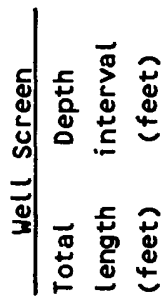

ț

홓 产 홓

홍 홍

홓 总 昰 总

홍 홍 홍 홍

18 索

品 ஜ

挙 点

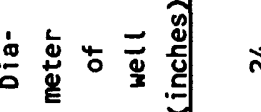

i

$\bar{\Sigma}$

in

宇

忿吕

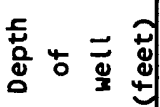

ํํํ

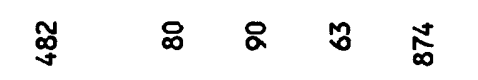

足 ำ ำ

๓

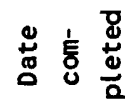

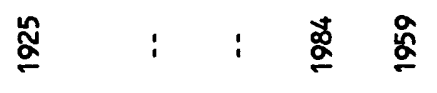

ڤ

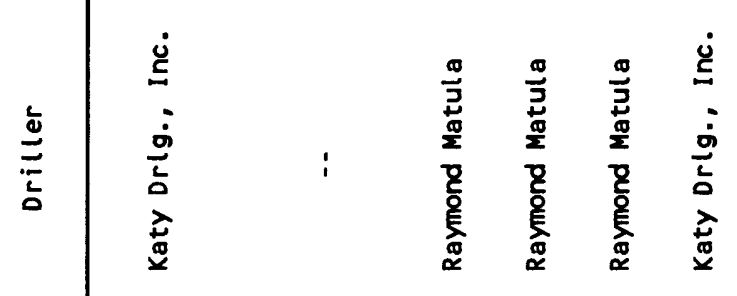

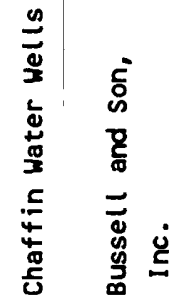

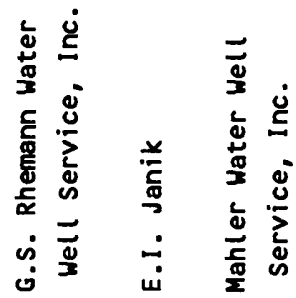

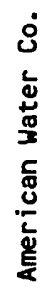

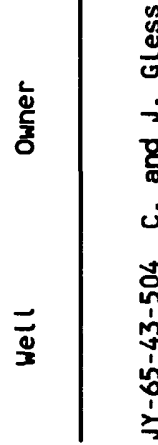

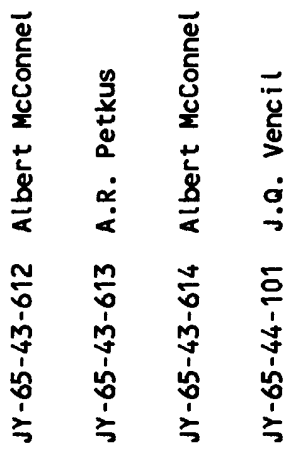

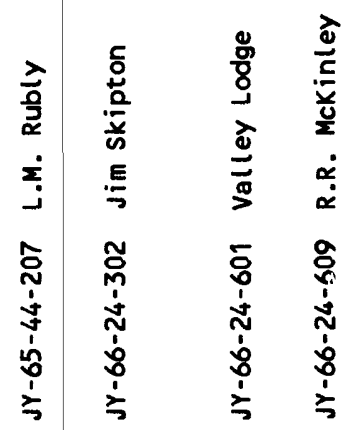

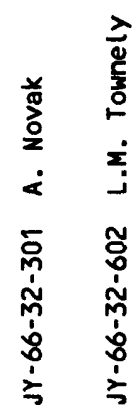

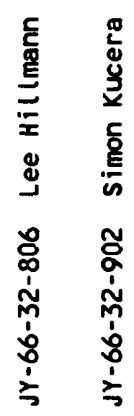




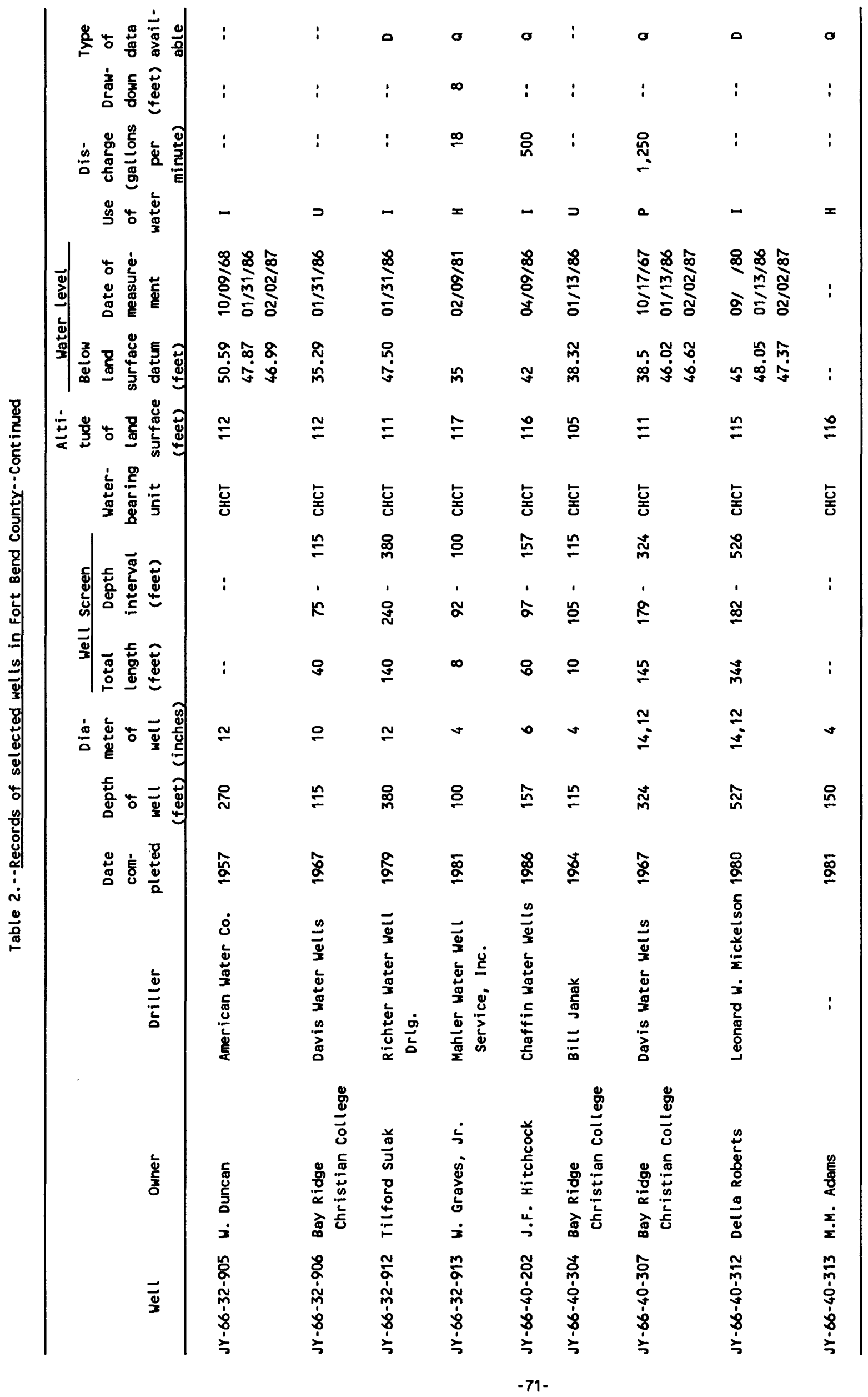


Well JY-65-10-810

Owner: Falcon Point

Driller: Bussell and Son, Inc.

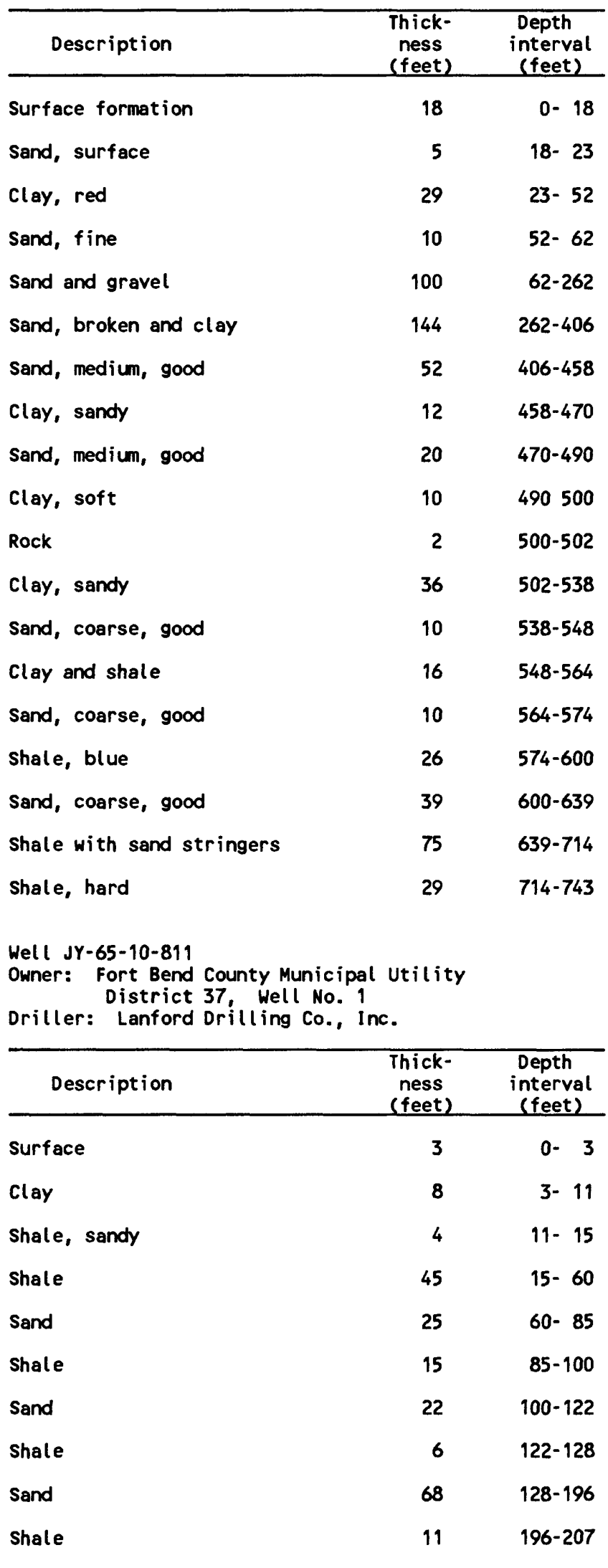

Well JY-65-10-811--Cont inued

\begin{tabular}{|c|c|c|c|}
\hline Sand & 78 & $207-$ & 285 \\
\hline Shale, sandy & 65 & $285-$ & 350 \\
\hline Sand & 30 & $350-$ & 380 \\
\hline Shale, sandy & 32 & $380-$ & 412 \\
\hline Sand & 50 & $412-$ & 462 \\
\hline Shale & 22 & $462-$ & 484 \\
\hline Sand & 20 & 484- & 504 \\
\hline Shale & 30 & 504- & 534 \\
\hline Sand & 20 & 534- & 554 \\
\hline Shale & 12 & $554-$ & 566 \\
\hline Sand & 44 & $566-$ & 610 \\
\hline shale & 12 & $610-$ & 622 \\
\hline Sand & 24 & $622-$ & 646 \\
\hline Shale & 22 & $646-$ & 668 \\
\hline Sand & 22 & $668-$ & 684 \\
\hline Shale & 56 & $684-$ & \\
\hline Sand & 30 & $740-$ & 770 \\
\hline Shale & 39 & $770-$ & 809 \\
\hline Sand & 116 & $809-$ & 925 \\
\hline Shale & 50 & $925-$ & 975 \\
\hline Sand & 37 & $975-1$ & 1,012 \\
\hline Shale & 54 & $1,012-1$ & \\
\hline Shale, sandy & 24 & $1,066-1$ & \\
\hline Shale & 75 & $1,090-1$ & \\
\hline Sand and sandy shale & 17 & $1,165-1$ & \\
\hline Shale & 30 & $1,182-1$ & \\
\hline Sand with shale streaks & 28 & $1,212-1$ & \\
\hline Shale & 67 & $1,240-1$ & \\
\hline
\end{tabular}

Well JY-65-10-812

Owner: City of Katy, Well No. 5

Driller: Layne-Western Co., Inc.

\begin{tabular}{lcr}
\hline Description & $\begin{array}{c}\text { Thick- } \\
\text { ness } \\
\text { (feet) }\end{array}$ & $\begin{array}{c}\text { Depth } \\
\text { interval } \\
\text { (feet) }\end{array}$ \\
\hline Topsoil & 3 & $0-3$ \\
Clay & 15 & $3-18$ \\
Sand, red & 5 & $18-23$ \\
Clay & 3 & $23-26$ \\
Sand & 4 & $26-30$
\end{tabular}


Well JY-65-10-812--Cont inued

\begin{tabular}{|c|c|c|}
\hline Clay & 47 & $30-77$ \\
\hline Sand & 20 & $77-97$ \\
\hline Clay & 23 & $97-120$ \\
\hline Sand & 50 & $120-170$ \\
\hline Clay & 20 & $170-190$ \\
\hline Sand & 13 & $190-203$ \\
\hline Clay & 10 & $203-213$ \\
\hline Sand & 63 & $213-276$ \\
\hline Clay & 3 & $276-279$ \\
\hline Sand & 7 & $279-286$ \\
\hline Clay & 26 & $286-312$ \\
\hline Sand & 12 & $312-324$ \\
\hline Clay & 11 & $324-335$ \\
\hline Rock and sand & 90 & $335-425$ \\
\hline Clay & 5 & $425-430$ \\
\hline Sand & 27 & $430-457$ \\
\hline Clay & 8 & $457-465$ \\
\hline Sand & 11 & $465-476$ \\
\hline Clay & 19 & $476-495$ \\
\hline Sand and clay & 105 & $495-600$ \\
\hline Clay & 26 & $600-626$ \\
\hline Sand & 16 & $626-642$ \\
\hline Clay & 33 & $642-675$ \\
\hline Sand & 11 & $675-686$ \\
\hline Clay & 39 & $686-725$ \\
\hline
\end{tabular}

Well JY-65-17-206

Owner: Richard A. Woods

Driller: Katy Drilling, Inc.

\begin{tabular}{lcr}
\hline \multicolumn{1}{c}{ Description } & $\begin{array}{c}\text { Thick- } \\
\text { ness } \\
\text { (feet) }\end{array}$ & $\begin{array}{c}\text { Depth } \\
\text { interval } \\
\text { (feet) }\end{array}$ \\
\hline Clay & 18 & $0-18$ \\
Clay and sand streaks & 7 & $18-25$ \\
Clay & 55 & $25-80$ \\
Sand and gravel & 43 & $80-123$ \\
Clay and sand streaks & 33 & $123-156$ \\
Sand and gravel & 44 & $156-200$ \\
Shale & 25 & $200-225$ \\
Sand and gravel & 15 & $225-240$
\end{tabular}

Well JY-65-17-206--Cont inued

Clay, hard streaks

$\begin{array}{rr}20 & 240-260 \\ 40 & 260-300 \\ 10 & 300-310 \\ 28 & 310-338 \\ 195 & 338-533 \\ 50 & 533-583\end{array}$

Well JY-65-17-308

Owner: Stewart and Stevenson, Inc.

Driller: Katy Drilling, Inc.

\begin{tabular}{|c|c|c|}
\hline Description & $\begin{array}{l}\text { Thick- } \\
\text { ness } \\
\text { (feet) }\end{array}$ & $\begin{array}{c}\text { Depth } \\
\text { interval } \\
\text { (feet) }\end{array}$ \\
\hline Surface and clay & 18 & $0-18$ \\
\hline Sand & 19 & 18- 37 \\
\hline Clay & 33 & $37-70$ \\
\hline Sand & 14 & $70-84$ \\
\hline Clay & 22 & $84-106$ \\
\hline Sand and gravel & 22 & $106-128$ \\
\hline Clay & 6 & $128-134$ \\
\hline Sand and gravel & 28 & $134-162$ \\
\hline Clay & 14 & $162-176$ \\
\hline Sand & 19 & $176-195$ \\
\hline Clay & 17 & $195-212$ \\
\hline Sand and rock with small clay breaks & 79 & $212-291$ \\
\hline Clay & 33 & $291-324$ \\
\hline Sand and rock & 30 & $324-354$ \\
\hline Clay & 18 & $354-372$ \\
\hline Sand and rock & 32 & $372-404$ \\
\hline Clay, tough & 57 & $404-461$ \\
\hline Sand and rock & 28 & $461-489$ \\
\hline Clay & 11 & $489-500$ \\
\hline Sand and rock & 15 & $500-515$ \\
\hline Clay & 26 & $515-541$ \\
\hline Sand and rock & 7 & $541-548$ \\
\hline Clay & 19 & $548-567$ \\
\hline Sand and rock & 56 & $567-623$ \\
\hline Clay & 9 & $623-632$ \\
\hline Sand and rock & 27 & $632-659$ \\
\hline Clay & 10 & $659-669$ \\
\hline
\end{tabular}


Well JY-65-17-308--Cont inued

\begin{tabular}{lcc} 
Sand & 42 & $669-711$ \\
Clay & 15 & $711-726$ \\
Sand and rock & 8 & $726-734$ \\
Clay & 45 & $734-779$ \\
Sand & 16 & $779-795$ \\
Clay & 5 & $795-800$ \\
Well JY-65-17-505 & & \\
Owner: Fort Bend County Municipal Utility & \\
Driller: Bussell and Son, Inc. & & \\
\hline & & \\
Description & Thick- & Depth \\
Clay & ness & interval \\
(feet)
\end{tabular}

\section{Well JY-65-17-606}

Owner: Stella Ranch

Driller: Katy Drilling, Inc.

\begin{tabular}{lcc}
\hline \multicolumn{1}{c}{ Description } & $\begin{array}{c}\text { Thick- } \\
\text { ness } \\
\text { (feet) }\end{array}$ & $\begin{array}{c}\text { Depth } \\
\text { interval } \\
\text { (feet) }\end{array}$ \\
\hline Clay, surface & 19 & $0-19$ \\
Sand & 21 & $19-40$ \\
Clay and sand & 18 & $40-58$ \\
Sand & 39 & $58-97$ \\
Clay & 7 & $97-104$ \\
Sand and gravel & 126 & $104-230$ \\
Clay and sand & 22 & $230-252$ \\
Sand and rock & 116 & $252-368$ \\
Clay & 11 & $368-379$ \\
Sand and rock & 10 & $379-389$ \\
Clay & 63 & $389-452$
\end{tabular}

Well JY-65-17-606--Cont inued

$\begin{array}{lcc}\text { Sand and rock } & 85 & 452-537 \\ \text { Clay } & 31 & 537-568 \\ \text { Sand and rock } & 81 & 568-649 \\ \text { Clay } & 123 & 649-772 \\ \text { Sand and rock } & 71 & 772-843 \\ \text { Clay } & 17 & 843-860 \\ \text { Sand } & 5 & 860-865 \\ \text { Clay } & 10 & 865-875\end{array}$

Well JY-65-17-607

Owner: Dennis Burke

Driller: G. S. Rhemann Water Well Service, Inc.

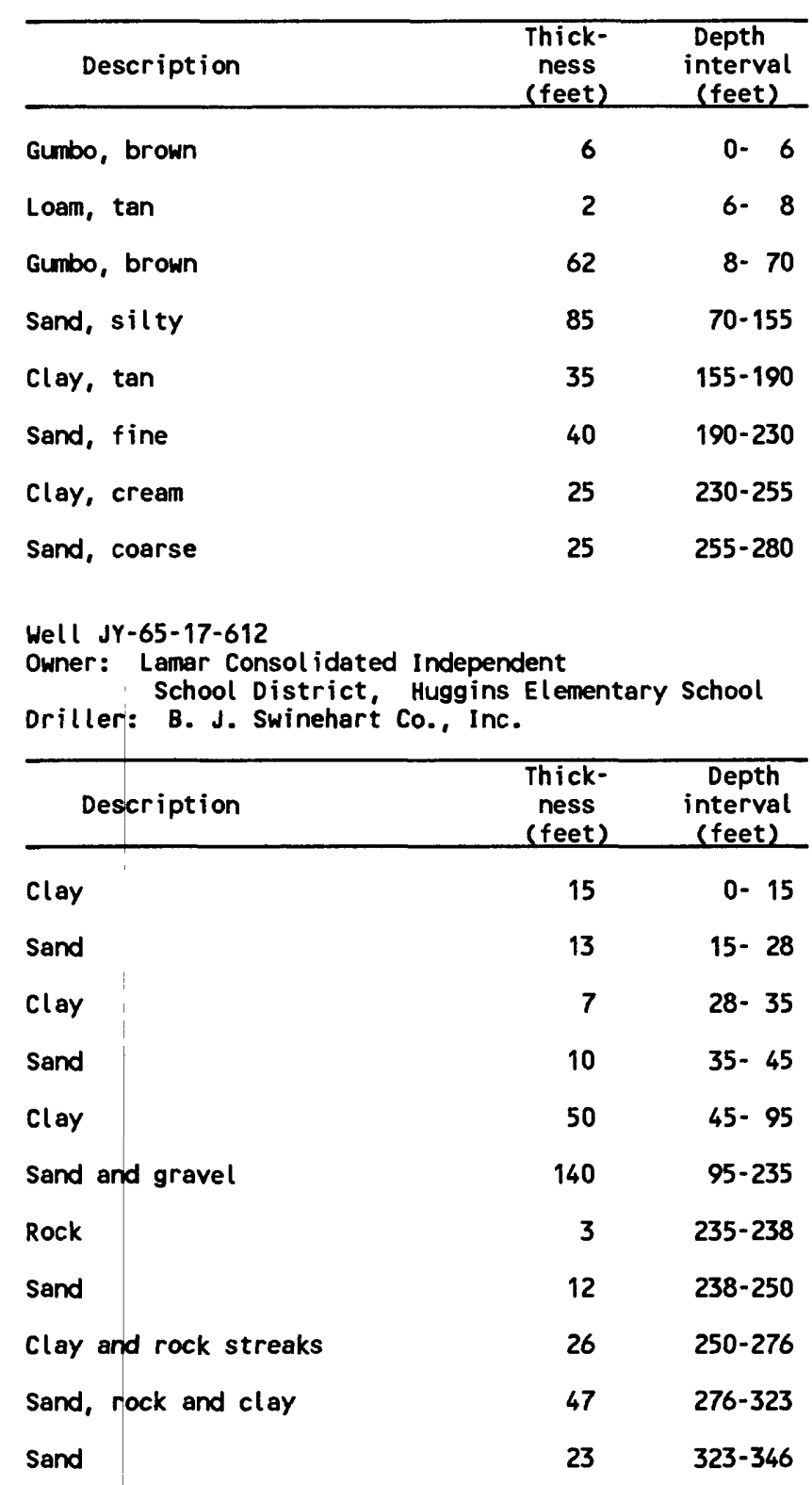


Well JY-65-17-612--Continued

Clay
Sand
Clay
Well JY-65-18-112
Owner: Cardiff Brothers
Driller: Katy Drilling, Inc.

Description

surface and clay

Sand

Clay

Sand

Clay

Sand, gravel and rock

Clay

Sand, hard rock

Clay

Sand, rock

Clay

Sand

Clay

Sand, rock

Clay

Sand, rock

Clay

Sand, rock

Clay

Sand

Clay

Sand, rock

Sand, rock

Clay

Sand

Sand

clay

Sand

Sand and shale
346-352

352-377

$377-378$

$\begin{array}{ll}\text { Thick- } & \text { Depth } \\ \text { ness } & \text { interval } \\ \text { feet) } & \text { (feet) }\end{array}$

28

$0-\quad 28$

$12 \quad 28-\quad 40$

$21 \quad 40-61$

$39 \quad 61-100$

$15 \quad 100-115$

$82 \quad 115-197$

$23 \quad 197-220$

$125 \quad 220-345$

$18 \quad 345-363$

33 363- 396

4 396- 400

5 400- 405

$40 \quad 405-445$

5 445- 450

$47 \quad 450-497$

$159 \quad 497-656$

$16 \quad 656-672$

$17 \quad 672-689$

$39 \quad 689-728$

16 728- 744

$16744-760$

$29 \quad 760-789$

$69 \quad 789-858$

$38 \quad 858-896$

$3 \quad 896-899$

15 899- 914

70 914- 984

$32984-1,016$

$371,016-1,053$
Well JY-65-18-113

Owner: Stewart and Stevenson, Inc.

Driller: Katy Drilling, Inc.

\begin{tabular}{|c|c|c|}
\hline Description & $\begin{array}{l}\text { Thick- } \\
\text { ness } \\
\text { (feet) }\end{array}$ & $\begin{array}{c}\text { Depth } \\
\text { interval } \\
\text { (feet) }\end{array}$ \\
\hline Topsoil & 52 & $0-52$ \\
\hline Sand & 34 & $52-86$ \\
\hline Clay & 14 & $86-100$ \\
\hline Sand & 28 & $100-128$ \\
\hline Clay & 6 & $128-134$ \\
\hline Sand, rocky with fine gravel & 92 & $134-226$ \\
\hline Clay & 4 & $226-230$ \\
\hline Sand with fine gravel & 76 & $230-306$ \\
\hline Clay & 6 & $306-312$ \\
\hline Sand, rocky with clay breaks & 40 & $312-352$ \\
\hline $\mathrm{Clay}$ and rock & 7 & $352-359$ \\
\hline Sand, rocky & 22 & $359-381$ \\
\hline Clay, soft with sand breaks & 97 & $381-478$ \\
\hline Sand and clay streaks & 10 & $478-488$ \\
\hline Clay & 26 & $488-514$ \\
\hline Sand, rock & 14 & $514-528$ \\
\hline Clay & 16 & $528-544$ \\
\hline Sand, rock & 68 & $544-612$ \\
\hline Clay & 16 & $612-628$ \\
\hline Sand, rock & 14 & $628-642$ \\
\hline Clay & 76 & $642-718$ \\
\hline Sand & 15 & $718-733$ \\
\hline Clay & 21 & $733-754$ \\
\hline Sand and rock & 14 & $754-768$ \\
\hline Clay & 35 & $768-803$ \\
\hline
\end{tabular}

Hell JY-65-18-207

Owner: E. K. Sanders

Driller: Katy Drilling, Inc.

\begin{tabular}{lcc}
\hline Description & $\begin{array}{c}\text { Thick- } \\
\text { ness } \\
\text { (feet) }\end{array}$ & $\begin{array}{c}\text { Depth } \\
\text { interval } \\
\text { (feet) }\end{array}$ \\
\hline Clay & 15 & $0-15$ \\
Sand & 5 & $15-20$ \\
Clay & 98 & $20-118$ \\
Sand & 22 & $118-140$ \\
Clay & 17 & $140-157$
\end{tabular}


Well JY-65-18-207--Continued

Sand and gravel
Clay
Sand and clay strips
Sand and gravel
Clay
Sand
Clay
Well JY-65-18-304
Owner: Cinco Ranch
Driller: Layne-Western Co., Inc.

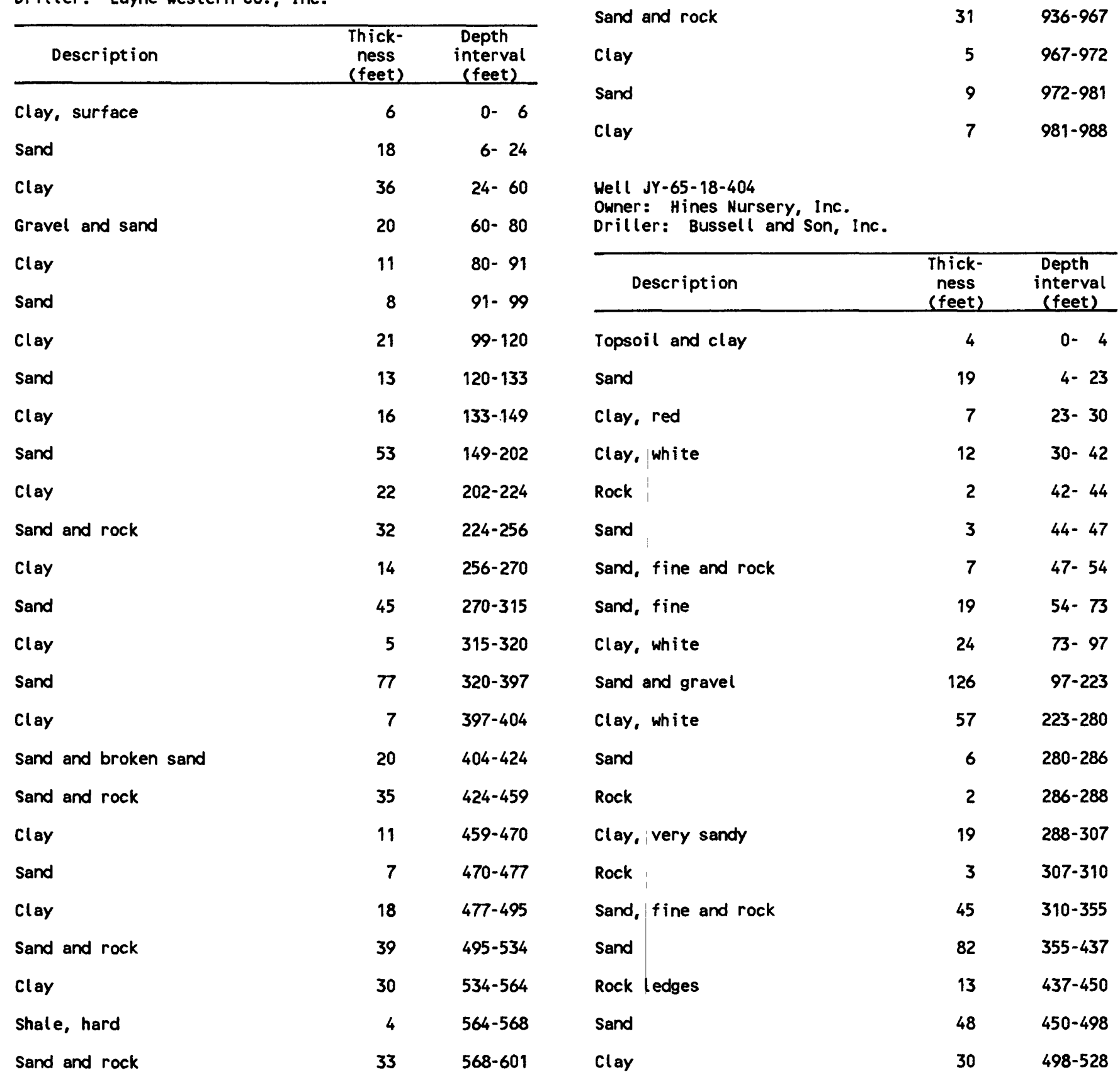

Well JY-65-18-304--Cont inued

$\begin{array}{ll}78 & 157-235 \\ 35 & 235-270 \\ 45 & 270-315 \\ 35 & 315-350 \\ 22 & 350-372 \\ 10 & 372-382 \\ 25 & 382-407\end{array}$

Clay

Sand and rock

44

601-645

Clay

5

$645-650$

Sand and rock

$51 \quad 650-701$

Clay

Sand and rock

34

$701-735$

Clay

Sand

Clay

7

89

$735-741$

71

741-830

830-901

5

901-906

30

906-936

clay

and rock

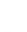


Hell JY-65-18-404--Continued

Sand
Clay, sandy
Sand
Clay, creme
Clay, sandy

Well JY-65-18-609

Owner: Fort Bend County Municipal Utility District 34

Driller: Layne-Western Co., Inc.

Description

Clay and sand

Sand

Clay

Sand and rock

Clay

Sand

Clay

Sand and gravel

Sand

Clay

Sand

clay

Sand

Clay

Sand

Clay

Sand

Clay

Sand

clay

Sand

Clay

Sand

Clay

Sand

clay

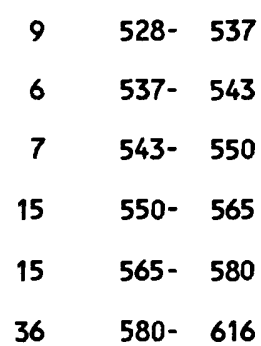

hick- $\begin{gathered}\text { Depth } \\ \text { ness }\end{gathered}$

feet) (feet)

$40 \quad 0-40$

$31 \quad 40-71$

$29 \quad 71-100$

$7 \quad 100-107$

$64 \quad 107-171$

$12 \quad 171 \cdot 183$

$3 \quad 183-186$

$29 \quad 186-215$

$225 \quad 215-440$

183 440- 623

$22 \quad 623-645$

$31 \quad 645-676$

6 676- 682

$7 \quad 682-689$

$18 \quad 689-707$

$40 \quad 707-747$

$19 \quad 747-766$

$62 \quad 766-828$

112 828- 940

7 940- 947

$6 \quad 947-953$

72 953-1,025

$171,025-1,042$

$48 \quad 1,042-1,090$

$601,090-1,150$

$50 \quad 1,150-1,200$
Hell JY-65-18-609--Cont inued

$\begin{array}{lcc}\text { Sand } & 90 & 1,200-1,290 \\ \text { Clay } & 18 & 1,290-1,308 \\ \text { Shale } & 37 & 1,308-1,345 \\ \text { Sand } & 83 & 1,345-1,428 \\ \text { Clay } & 72 & 1,428-1,500\end{array}$

Hell JY-65-18-610

Owner: Tom Peckinpaugh

Driller: Mahler Hater Well Service, Inc.

\begin{tabular}{|c|c|c|c|}
\hline \multirow{2}{*}{$\begin{array}{r}\text { Description } \\
\text { Topsoil }\end{array}$} & \multirow{2}{*}{$\begin{array}{c}\begin{array}{c}\text { Thick- } \\
\text { ness } \\
\text { (feet) }\end{array} \\
5\end{array}$} & \multicolumn{2}{|c|}{$\begin{array}{c}\text { Depth } \\
\text { interval } \\
\text { (feet) }\end{array}$} \\
\hline & & $0-$ & 5 \\
\hline Clay, white & 10 & $5-$ & 15 \\
\hline Sand & 10 & 15- & 25 \\
\hline clay, red & 30 & $25-$ & 55 \\
\hline Shale, white & 15 & $55-$ & 70 \\
\hline Sand & 20 & $70-$ & 90 \\
\hline Shale, blue & 15 & $90-$ & 105 \\
\hline Sand & 5 & $105-$ & 110 \\
\hline Shale, white & 5 & $110-$ & 115 \\
\hline Sand & 25 & $115-$ & 140 \\
\hline Shale & 10 & $140-$ & 150 \\
\hline Sand & 65 & $150-$ & 215 \\
\hline Sand and shale & 30 & $215-$ & 245 \\
\hline Sand & 30 & 245 & 275 \\
\hline Shale & 5 & $275-$ & 280 \\
\hline Sand and shale & 25 & $280-$ & 305 \\
\hline Sand & 25 & $305-$ & 330 \\
\hline $\begin{array}{l}\text { Well JY-65-18-705 } \\
\text { Owner: Bart Vickers }\end{array}$ & Service, & Inc. & \\
\hline Description & $\begin{array}{l}\text { Thick- } \\
\text { ness } \\
\text { (feet) }\end{array}$ & $\begin{array}{r}\text { Dep } \\
\text { inte } \\
\text { (fe }\end{array}$ & $\begin{array}{l}\text { th } \\
\text { rval } \\
\text { et) }\end{array}$ \\
\hline Topsoil & 3 & $0-$ & 3 \\
\hline Sand, tan & 12 & $3-$ & 15 \\
\hline Loam, tan & 24 & $15-$ & 39 \\
\hline Sand & 24 & $39-$ & 63 \\
\hline Rock & 3 & 63- & 66 \\
\hline Clay, tan & 28 & 66- & 94 \\
\hline
\end{tabular}


Well JY-65-18-705--Cont inued

Gravel
Rock
Clay, reddi sh-brown
Sand
Rock
Clay, hard
Clay, rocky
Sand, hard
Sand, coarse
Well Jy-65-18-706
Owner: Harrison Interests Ltd.
Driller: Mueller Well Service

\begin{tabular}{lcc}
\hline \multicolumn{1}{c}{ Description } & $\begin{array}{c}\text { Thick- } \\
\text { ness } \\
\text { (feet) }\end{array}$ & $\begin{array}{c}\text { Depth } \\
\text { interval } \\
\text { (feet) }\end{array}$ \\
\hline Sand & 9 & $0-9$ \\
Clay, red & 32 & $9-41$ \\
Sand & 13 & $41-54$ \\
Gravel & 11 & $54-65$ \\
Sand and yellow clay & 25 & $65-90$ \\
Sand & 22 & $90-112$ \\
Sand and gravel & 24 & $112-136$ \\
Sands tone & 1 & $136-137$ \\
Sand & 38 & $137-175$
\end{tabular}

Well JY-65-18-905

Owner: Jerry Lea

Driller: G. S. Rhemann Water Well Service, Inc.

\begin{tabular}{lcc}
\hline \multicolumn{1}{c}{ Description } & $\begin{array}{c}\text { Thick- } \\
\text { ness } \\
\text { (feet) }\end{array}$ & $\begin{array}{c}\text { Depth } \\
\text { interval } \\
\text { (feet) }\end{array}$ \\
\hline Topsoil & 4 & $0-4$ \\
Clay, brown & 18 & $4-22$ \\
Sand and gravel & 23 & $22-45$ \\
Clay, gray & 10 & $45-55$ \\
Sand and gravel & 5 & $55-60$ \\
Sandstone & 1 & $60-61$ \\
Clay, brown & 23 & $61-84$ \\
Sand & 10 & $84-94$ \\
Clay, brown & 16 & $94-110$ \\
Clay, rocky & 10 & $110-120$
\end{tabular}

Well JY-65-18-905--Cont inued

$\begin{array}{lcc}\text { Gravel and sand } & 18 & 120-138 \\ \text { Clay, brown } & 2 & 138-140 \\ \text { Sand, rocky } & 32 & 140-172 \\ \text { Stone layers } & 28 & 172-200 \\ \text { Clay, gray } & 16 & 200-216 \\ \text { Sand, coarse } & 44 & 216-260\end{array}$

Well JY-65-19-406

Owner: Castone Brick

Driller: Raymond Water Wells

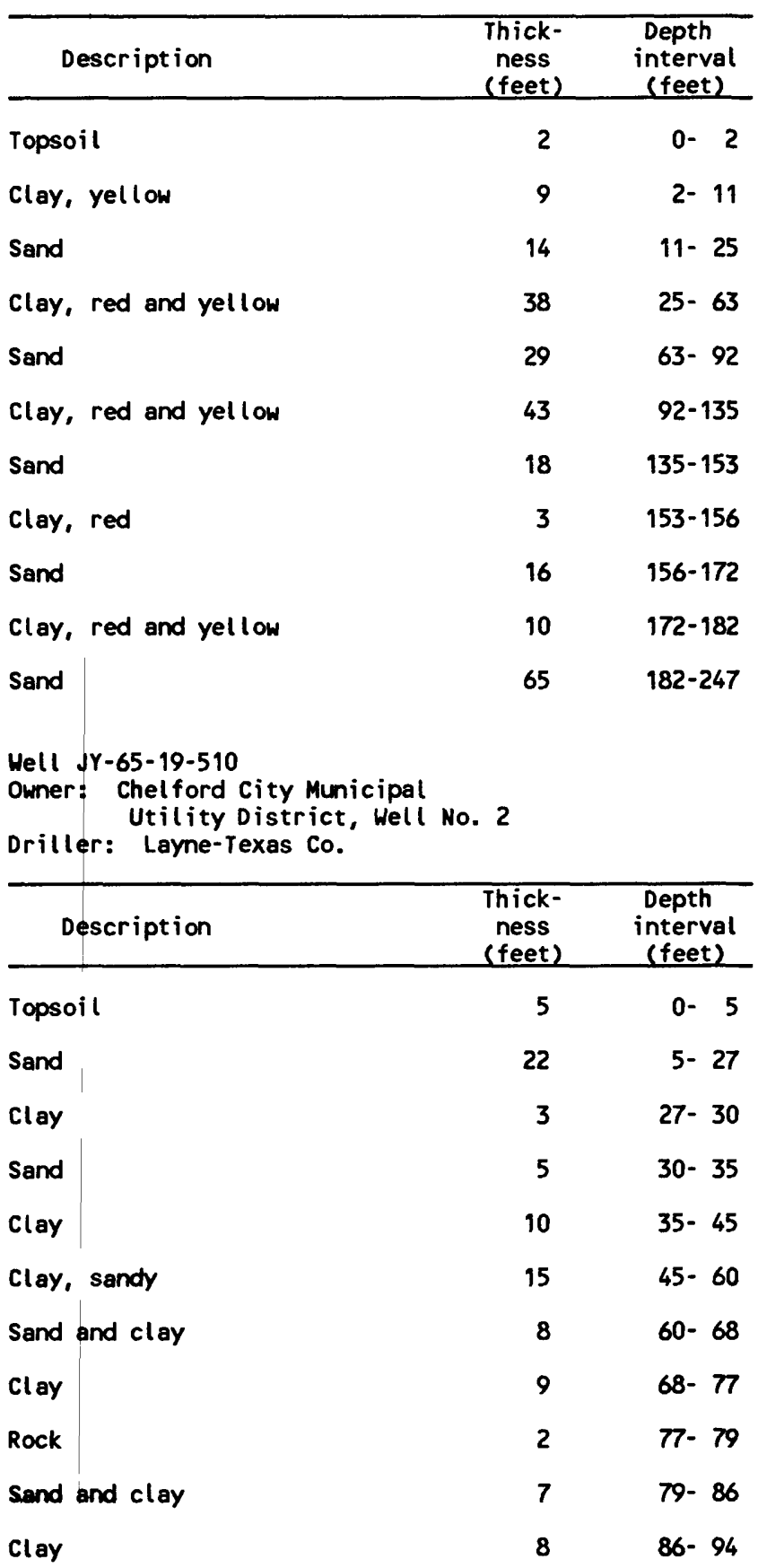


Well JY-65-19-510--Cont inued

\begin{tabular}{|c|c|c|}
\hline Sand and clay & 118 & $94-212$ \\
\hline Sand and shale & 134 & $212-346$ \\
\hline Sand & 10 & $346-356$ \\
\hline Sand and shale & 34 & $356-390$ \\
\hline Shale and sand & 68 & $390-458$ \\
\hline Sand & 42 & $458-500$ \\
\hline Shale & 31 & $500-531$ \\
\hline Sand & 24 & $531-555$ \\
\hline Shale & 12 & $555-567$ \\
\hline Sand & 10 & $567-577$ \\
\hline Shate & 12 & $577-589$ \\
\hline Shale, hard, sandy & 55 & $589-644$ \\
\hline Sand & 6 & $644-650$ \\
\hline Sand and shate & 56 & $650-706$ \\
\hline Shale and sand streaks & 5 & $706-711$ \\
\hline Sand, shale and lime & 35 & $711-746$ \\
\hline Shale & 12 & $746-758$ \\
\hline Sand and lime streaks & 7 & $758-765$ \\
\hline Shale & 9 & $765-774$ \\
\hline Sand & 11 & $774-785$ \\
\hline Sand, shale and lime & 84 & $785-869$ \\
\hline Sand & 11 & $869-880$ \\
\hline \multicolumn{3}{|c|}{$\begin{array}{l}\text { Well JY- } 65-19-511 \\
\text { Owner: Dunaway } \\
\text { Driller: Robinson Water Well Service, Inc. }\end{array}$} \\
\hline Description & $\begin{array}{l}\text { Thick- } \\
\text { ness } \\
\text { (feet) }\end{array}$ & $\begin{array}{l}\text { Depth } \\
\text { interval } \\
\text { (feet) } \\
\end{array}$ \\
\hline Clay & 15 & $0-15$ \\
\hline Sand & 21 & $15-36$ \\
\hline Clay & 127 & $36-163$ \\
\hline Sand & 37 & $163-200$ \\
\hline Clay & 40 & $200-240$ \\
\hline Sand & 53 & $240-293$ \\
\hline
\end{tabular}

Well JY-65-19-512

Owner: Dunaway

Driller: Robinson Water Well Service, Inc.

\begin{tabular}{lcc}
\hline Description & $\begin{array}{c}\text { Thick- } \\
\text { ness } \\
\text { (feet) }\end{array}$ & $\begin{array}{c}\text { Depth } \\
\text { interval } \\
\text { (feet) }\end{array}$ \\
\hline Clay & 12 & $0-12$ \\
Sand & 62 & $12-74$ \\
Clay & 59 & $74-133$ \\
Sand & 107 & $133-240$ \\
Clay & 30 & $240-270$ \\
Sand & 50 & $270-320$ \\
Clay & 30 & $320-350$ \\
Sand & 41 & $350-391$
\end{tabular}

Well JY-65-19-513

Owner: Big Oak Municipal Utility District

Driller: Layne-Texas Co.

\begin{tabular}{|c|c|c|}
\hline Description & $\begin{array}{l}\text { Thick- } \\
\text { ness } \\
\text { (feet) }\end{array}$ & $\begin{array}{c}\text { Depth } \\
\text { interval } \\
\text { (feet) }\end{array}$ \\
\hline Topsoil & 2 & $0-2$ \\
\hline Clay & 70 & 2- 72 \\
\hline Sand & 31 & $72-103$ \\
\hline Clay & 13 & $103-116$ \\
\hline Sand and gravel & 156 & $116-272$ \\
\hline Shale & 34 & $272-306$ \\
\hline Sand and shale streaks & 34 & $306-340$ \\
\hline Shale & 20 & $340-360$ \\
\hline Sand and shale streaks & 46 & $360-406$ \\
\hline Shale and sand streaks & 11 & $406-417$ \\
\hline Sand & 38 & $417-455$ \\
\hline Shale and lime streaks & 37 & $455-492$ \\
\hline Sand with few shale and lime streaks & 94 & $492-586$ \\
\hline Shale and lime streaks & 17 & $586-603$ \\
\hline Shale, sandy & 34 & $603-637$ \\
\hline Shale & 5 & $637-642$ \\
\hline Lime & 15 & $642-657$ \\
\hline Sand and lime streaks & 34 & $657-691$ \\
\hline Lime & 6 & $691-697$ \\
\hline Sand & 20 & $697-717$ \\
\hline Shate & 23 & $740-740$ \\
\hline
\end{tabular}


Well JY-65-19-513--Cont inued

$\begin{array}{llll}\text { Sand } & 31 & 740- & 771 \\ \text { Shale and sand streaks } & 81 & 771-852 \\ \text { Sand and shale streaks } & 18 & 852-870 \\ \text { Shale and sand streaks } & 50 & 870-920 \\ \text { Sand and shale streaks } & 34 & 920-954 \\ \text { Shale } & 34 & 954-988 \\ \text { Sand and shale streaks } & 18 & 988-1,006 \\ \text { Shale } & 94 & 1,006-1,100\end{array}$

Well JY-65-19-601

Owner: John L. Dore Company

Driller: Layne-Texas Co.

\begin{tabular}{|c|c|c|c|}
\hline Description & $\begin{array}{l}\text { Thick- } \\
\text { ness } \\
\text { (feet) }\end{array}$ & $\begin{array}{l}\text { Dep } \\
\text { inte } \\
\text { Cfe }\end{array}$ & th \\
\hline Topsoil & 2 & 0 & 2 \\
\hline Clay, yellow & 4 & $2-$ & 6 \\
\hline Clay, red & 18 & 6- & 24 \\
\hline Clay, yellow & 26 & $24-$ & 50 \\
\hline Clay, red and yellow & 14 & $50-$ & 64 \\
\hline Clay, red & 20 & $64-$ & 84 \\
\hline clay, yellow & 10 & 84- & 94 \\
\hline Clay, gray sandy & 31 & $94-$ & 125 \\
\hline Clay, yellow & 29 & $125-$ & 154 \\
\hline clay, gray & 10 & 154- & 164 \\
\hline Clay, sandy & 13 & $164-$ & 177 \\
\hline Gravel and sand & 8 & $177-$ & 185 \\
\hline Sand and gravel & 11 & $185-$ & 196 \\
\hline $\begin{array}{l}\text { Sand and gravel, clay streaks and } \\
\text { rock streaks }\end{array}$ & 49 & 196- & 245 \\
\hline Sand and gravel and clay streaks & 8 & $245-$ & 253 \\
\hline Clay and sandy clay & 5 & 253- & 258 \\
\hline Sand and gravel and clay streaks & 47 & $258-$ & 305 \\
\hline Rock and clay streaks & 5 & $305-$ & 310 \\
\hline Clay, sandy and clay & 21 & $310-$ & 331 \\
\hline Clay and gravel streaks & 31 & $331-$ & 362 \\
\hline Sand stone and clay streaks & 21 & $362-$ & 383 \\
\hline clay and sandy clay & 22 & $383-$ & 405 \\
\hline Clay and few gravel streaks & 28 & $405-$ & 433 \\
\hline Clay and gravel & 10 & 433- & 443 \\
\hline
\end{tabular}

Well JY-65-19-601--Cont inued

$\begin{array}{lrr}\text { Clay and layers of gravel } & 13 & 443-456 \\ \text { Clay, sandy } & 7 & 456-463 \\ \text { Sand and gravel and clay streaks } & 21 & 463-484 \\ \text { Clay } & 3 & 484-487 \\ \text { Sand and gravel and clay streaks } & 7 & 487-494 \\ \text { Sand, fine } & 6 & 494-500 \\ \text { Clay and gravel } & 7 & 500-507 \\ \text { Rock } & 1 & 507-508 \\ \text { Sand, fine and few hard streaks } & 7 & 508-515 \\ \text { Clay and gravel } & 9 & 515-524 \\ \text { Sand and few streaks of clay } & 18 & 524-542 \\ \text { Clay } & 13 & 542-555\end{array}$

Well JY-65-19-603

Owner: Kingsbridge Municipal Utility District, Well No. 1

Driller: Layne-Texas Co.

\begin{tabular}{|c|c|c|}
\hline Description & $\begin{array}{l}\text { Thick- } \\
\text { ness } \\
\text { (feet) }\end{array}$ & $\begin{array}{c}\text { Depth } \\
\text { interval } \\
\text { (feet) }\end{array}$ \\
\hline Gumbo & 7 & $0-7$ \\
\hline Clay & 19 & 7- 26 \\
\hline Sand & 9 & 26- 35 \\
\hline Clay & 55 & $35-90$ \\
\hline Sand & 32 & $90-122$ \\
\hline Clay and sand streaks & 24 & $122-146$ \\
\hline Sand & 39 & $146-185$ \\
\hline Shale & 17 & $185-202$ \\
\hline Sand and shale & 61 & $202-263$ \\
\hline Rock & 8 & $263-271$ \\
\hline Shale & 15 & $271-286$ \\
\hline Shale and sand streaks & 10 & $286-296$ \\
\hline Shale & 60 & $296-356$ \\
\hline Shale and sand streaks & 25 & $356-381$ \\
\hline Sand & 14 & $381-395$ \\
\hline Shale & 26 & $395-421$ \\
\hline Shale and sand streaks & 18 & $421-439$ \\
\hline Sand and shale streaks & 30 & $439-469$ \\
\hline Shale & 12 & $469-481$ \\
\hline Shale, sandy and sand & 49 & $481-530$ \\
\hline
\end{tabular}


Hell JY-65-19-603--Cont inued

$\begin{array}{lrr}\text { Sand and shale streaks } & 211 & 530-741 \\ \text { Shale } & 39 & 741-780 \\ \text { Sand and shale } & 74 & 780-854 \\ \text { Shale } & 9 & 854-863 \\ \text { Sand and shale streaks } & 54 & 863-917 \\ \text { Shale and sand streaks } & 61 & 917-978 \\ \text { Sand } & 4 & 978-982 \\ \text { Shale and sand streaks } & 62 & 982-1,044 \\ \text { Shale } & 100 & 1,044-1,144 \\ \text { Shale and sand } & 9 & 1,144-1,153 \\ \text { Sand and shale } & 31 & 1,153-1,184 \\ \text { Shale and sand streaks } & 111 & 1,184-1,295 \\ \text { Sand and shale } & 35 & 1,295-1,330 \\ \text { Shale and sand streaks } & 40 & 1,330-1,370 \\ \text { Sand and shale } & 60 & 1,370-1,430 \\ \text { Shale } & 20 & 1,430-1,450 \\ \text { Shale and sand } & 36 & 1,450-1,486 \\ \text { Shale } & 214 & 1,486-1,700\end{array}$

Hell JY-65-19-604

Owner: Tree and Hood Disposal

Driller: G. S. Rhemann Hater Hell Service, Inc.

\begin{tabular}{|c|c|c|c|c|c|c|c|}
\hline \multirow{2}{*}{$\begin{array}{l}\text { Description } \\
\text { Gumbo }\end{array}$} & \multirow{2}{*}{$\begin{array}{c}\text { Thick- } \\
\text { ness } \\
\text { (feet) }\end{array}$} & \multicolumn{2}{|c|}{$\begin{array}{c}\text { Depth } \\
\text { interval } \\
\text { (feet) } \\
\end{array}$} & \multirow{2}{*}{$\begin{array}{l}\text { Clay, grayish white } \\
\text { Sand } \\
\text { Clay, grayish white }\end{array}$} & \multirow{2}{*}{$\begin{array}{r}20 \\
170 \\
20\end{array}$} & \multirow{2}{*}{$\begin{array}{l}444- \\
464- \\
634-\end{array}$} & \multirow{2}{*}{$\begin{array}{l}464 \\
634 \\
654\end{array}$} \\
\hline & & 0 - & 3 & & & & \\
\hline Clay, tan & 25 & 3- & 28 & Sand & 20 & $654-$ & 674 \\
\hline Sand & 7 & 28- & 35 & Clay, grayish white & 20 & $674-$ & 694 \\
\hline Clay & 5 & $35-$ & 40 & Sand & 60 & $694-$ & 754 \\
\hline Clay, $\tan$ and blue & 20 & $40-$ & 60 & Clay, grayish white & 15 & $754-$ & 769 \\
\hline Sand & 16 & $60-$ & 76 & Sand & 45 & $769-$ & 814 \\
\hline Clay, red & 4 & $76-$ & 80 & Clay, gray & 20 & $814-$ & 834 \\
\hline clay, blue & 10 & $80-$ & 90 & Sand & 20 & $834-$ & 854 \\
\hline Sand, rocky & 10 & $90-$ & 100 & Clay, $\tan$ & 10 & 854- & 864 \\
\hline Sand & 26 & $100-$ & 126 & Sand & 40 & $864-$ & 904 \\
\hline Clay, blue & 6 & $126-$ & 132 & Clay, gray & 110 & $904-1$ & .014 \\
\hline Rock and clay & 10 & $132-$ & 142 & Sand & 30 & $1,014-1$ & .044 \\
\hline Sand & 26 & $142-$ & 168 & Clay, gray & 30 & $1,044-1$ & .074 \\
\hline Clay, red and blue & 29 & $168-$ & 197 & Sand & 40 & $1,074-1$ & 114 \\
\hline Sand & 43 & $197-$ & 240 & Clay, gray & 30 & $1,114-1$ &, 144 \\
\hline
\end{tabular}

Well JY-65-19-606

Owner: North Mission Glen Municipal

Utility District, Hell No. 2

\begin{tabular}{|c|c|c|c|}
\hline Description & $\begin{array}{l}\text { Thick- } \\
\text { ness } \\
\text { (feet) }\end{array}$ & $\begin{array}{r}\text { Dep } \\
\text { inte } \\
\text { (fe }\end{array}$ & $\begin{array}{l}\text { th } \\
\text { rval } \\
\text { et) }\end{array}$ \\
\hline Topsoil & 4 & 0- & 4 \\
\hline Sand & 13 & 4- & 17 \\
\hline Clay, red & 22 & $17-$ & 39 \\
\hline Sand & 19 & 39- & 58 \\
\hline $\mathrm{Clay}$, red & 46 & $58-$ & 104 \\
\hline Sand & 16 & 104- & 120 \\
\hline Clay, grayish white & 10 & $120-$ & 130 \\
\hline Sand and soft gravel & 80 & $130-$ & 210 \\
\hline Clay, gray & 24 & $210-$ & 234 \\
\hline Sand, hard & 15 & 234- & 249 \\
\hline Clay, gray & 19 & 249- & 268 \\
\hline Sand & 22 & 268- & 290 \\
\hline Sand with small clay streaks & 24 & $290-$ & 314 \\
\hline Clay, gray & 30 & $314-$ & 344 \\
\hline Sand & 10 & 344- & 354 \\
\hline Clay, gray & 40 & $354-$ & 394 \\
\hline Sand & 50 & 394- & 444 \\
\hline Clay, grayish white & 20 & $444-$ & 464 \\
\hline Sand & 170 & 464- & 634 \\
\hline Clay, grayish white & 20 & $634-$ & 654 \\
\hline Sand & 20 & 654- & 674 \\
\hline Clay, grayish white & 20 & $674-$ & 694 \\
\hline Sand & 60 & $694-$ & 754 \\
\hline Clay, grayish white & 15 & $754-$ & 769 \\
\hline Sand & 45 & $769-$ & 814 \\
\hline Clay, gray & 20 & $814-$ & 834 \\
\hline Sand & 20 & 834- & 854 \\
\hline Clay, $\tan$ & 10 & 854- & 864 \\
\hline Sand & 40 & $864-$ & 904 \\
\hline Clay, gray & 110 & \multicolumn{2}{|c|}{$904-1,014$} \\
\hline Sand & 30 & \multicolumn{2}{|c|}{$1,014-1,044$} \\
\hline Clay, gray & 30 & \multicolumn{2}{|c|}{$1,044-1,074$} \\
\hline Sand & 40 & \multicolumn{2}{|c|}{$1,074-1,114$} \\
\hline Clay, gray & 30 & \multicolumn{2}{|c|}{$1,114-1,144$} \\
\hline
\end{tabular}

Driller: Alsay Incorporated 
Hell JY-65--19-606--Continued

Sand, hard

Clay, gray

Sand

Clay, sandy

Sand with small clay streaks

Clay, gray and all hard sand streaks $20 \quad 1,374-1,394$

Hell JY-65-19-706

Owner: Pecan Grove Municipal Utility District 2, Hell No. 1

Driller: Layne-Texas Co.

\begin{tabular}{|c|c|c|c|}
\hline Description & $\begin{array}{l}\text { Thick- } \\
\text { ness } \\
\text { (feet) }\end{array}$ & $\begin{array}{l}\text { Dep } \\
\text { inte } \\
\text { (fe }\end{array}$ & $\begin{array}{l}\text { th } \\
\text { rval } \\
\text { et) }\end{array}$ \\
\hline Clay, red & 17 & $0-$ & 17 \\
\hline Sand, coarse & 49 & $17-$ & 66 \\
\hline Shale & 16 & 66- & 82 \\
\hline Sand, coarse with gravel & 40 & 82- & 122 \\
\hline Clay, red with sand streaks & 26 & $122-$ & 148 \\
\hline Sand & 9 & $148-$ & 157 \\
\hline Gravel and sand streaks & 44 & $157-$ & 201 \\
\hline Shale, sandy & 8 & $201-$ & 209 \\
\hline Gravel & 31 & 209- & 240 \\
\hline Sand & 7 & $240-$ & 247 \\
\hline Shale & 33 & $247-$ & 280 \\
\hline Sand & 52 & $280-$ & 332 \\
\hline Shale & 7 & $332-$ & 339 \\
\hline Sand with shale streaks & 48 & $339-$ & 387 \\
\hline Shale & 21 & $387-$ & 408 \\
\hline Sand with shale streaks & 36 & 408- & 444 \\
\hline Shale & 5 & 444- & 449 \\
\hline $\begin{array}{l}\text { Sand, coarse with gravel and } \\
\text { shale streaks }\end{array}$ & 109 & 449- & 558 \\
\hline Shale, hard & 16 & $558-$ & 574 \\
\hline Shale, soft & 21 & 574- & 595 \\
\hline Sand & 38 & $595-$ & 633 \\
\hline Shale & 8 & 633- & 641 \\
\hline Sand and gravel & 6 & 641 - & 647 \\
\hline Limestone & 2 & $647-$ & 649 \\
\hline Sand & 36 & 649- & 685 \\
\hline Shale, soft & 17 & $685-$ & 702 \\
\hline
\end{tabular}

$251,154-1,179$

$25 \quad 1,179-1,204$

$101,204-1,214$
Hell JY-65-19-706--Cont inued

Shale, sandy 17

Shale and little gravel

702- 719

Sand

$17 \quad 719-736$

shele

19

736- 755

Sand

24

$755-779$

38

779- 817

Sand and Iime streaks

Lime, hard

817- 825

Shale, sticky

$825-827$

Shale with sand streaks

14

$827-841$

Sand with shale streaks

13

Shale, sticky

39

841- 854

Shale, hard

14

854- 893

Shale, sandy

7

893- 907

Sand and shale streaks

43

$907-914$

Shale

13

Shale, sticky

9

Shale, sandy

10

71

$989-1,060$

Sand

$48 \quad 1,060-1,108$

Shale

$7 \quad 1,108-1,115$

Sand with gravel and shale streaks

$16 \quad 1,115-1,131$

Shale

$15 \quad 1,131-1,146$

Shale, sandy and gravel

$25 \quad 1,146-1,171$

Shale

Shale, sandy with gravel

$7 \quad 1,171-1,178$

31 1,178-1,209

Sand

$14 \quad 1,209-1,223$

Shale, sandy

$25 \quad 1,223-1,248$

Sand

Shale, sandy

Shale

Sand

Shale

Sand

Shale

Shale, sandy

$10 \quad 1,248-1,258$

$14 \quad 1,258-1,272$

$6 \quad 1,272-1,278$

$35 \quad 1,278-1,313$

$24 \quad 1,313-1,337$

$6 \quad 1,337-1,343$

$25 \quad 1,343-1,368$

$281,368-1,396$

Sand

$21 \quad 1,396-1,417$

Shale

$20 \quad 1,417-1,437$

Sand

$15 \quad 1,437-1,452$

Shale

$56 \quad 1,452-1,508$ 
Well JY-65-19-807

Owner: Texas Department of Corrections Beuford Jester Unit No. 5

Driller: Layne-Texas Co.

\begin{tabular}{|c|c|c|}
\hline Description & $\begin{array}{c}\text { Thick- } \\
\text { ness } \\
\text { (feet) }\end{array}$ & $\begin{array}{c}\text { Depth } \\
\text { interval } \\
\text { (feet) }\end{array}$ \\
\hline Topsoil & 2 & 0- \\
\hline Clay, red & 43 & 2- \\
\hline Sand, coarse and gravel & 140 & 45- $\quad 185$ \\
\hline Clay & 113 & $185-298$ \\
\hline $\mathrm{Clay}$ and sand streaks & 86 & $298-384$ \\
\hline Sand, coarse and gravel & 157 & $384-541$ \\
\hline $\begin{array}{l}\text { Sand, coarse, gravel and clay with } \\
\text { lime streaks }\end{array}$ & 86 & $541-627$ \\
\hline Shale and sand streaks & 46 & $627-673$ \\
\hline Sand & 59 & $673-732$ \\
\hline Shale and sand streaks & 18 & $732-750$ \\
\hline Sand & 57 & $750-807$ \\
\hline Shale, sand, and gravel streaks & 78 & $807-885$ \\
\hline Sand & 22 & $885-907$ \\
\hline Shale and lime streaks & 15 & $907-922$ \\
\hline Shale, lime, and sand & 29 & $922-951$ \\
\hline Sand and shale streaks & 80 & $951-1,031$ \\
\hline Shale and lime & 7 & $1,031-1,038$ \\
\hline Shale and sand streaks & 10 & $1,038-1,048$ \\
\hline Sand & 10 & $1,048-1,058$ \\
\hline Shale, sandy & 13 & $1,058-1,071$ \\
\hline Sand & 66 & $1,071-1,137$ \\
\hline Shale & 3 & $1,137-1,140$ \\
\hline Sand and shale streaks & 81 & $1,140-1,221$ \\
\hline
\end{tabular}

Well JY-65-19-809

Owner: Fort Bend County Municipal Utility District 25, Well No. 1

Driller: Water Resources of Texas

\begin{tabular}{lcc}
\hline \multicolumn{1}{c}{ Description } & $\begin{array}{c}\text { Thick- } \\
\text { ness } \\
\text { (feet) }\end{array}$ & $\begin{array}{c}\text { Depth } \\
\text { interval } \\
\text { (feet) }\end{array}$ \\
\hline Unrecorded & 500 & $0-500$ \\
Shale, sandy & 15 & $500-515$ \\
Sand, good & 145 & $515-660$ \\
Sand and sandy shale & 20 & $660-680$ \\
Sand, good & 60 & $680-740$ \\
Shale, sandy & 15 & $740-755$
\end{tabular}

Well JY-65-19-809--Continued

Sand, good

755- 785

Shale, sandy

$30 \quad 785-815$

Sand, good

25

815- 840

Shale breaks, sandy

$20 \quad 840-860$

Sand and sandy shale

20

860- 880

Shale breaks, sandy

70

$880-950$

Shale, some hard

100

$950-1,050$

Well JY-65-19-904

Owner: Fort Bend County Hater Control and Improvement District 4

Driller: Layne-Texas Co.

\begin{tabular}{|c|c|c|c|}
\hline Description & $\begin{array}{l}\text { Thick- } \\
\text { ness } \\
\text { (feet) }\end{array}$ & $\begin{array}{l}\text { Dep } \\
\text { inte } \\
\text { (fe }\end{array}$ & $\begin{array}{l}\text { th } \\
\text { rval } \\
\text { et) }\end{array}$ \\
\hline Topsoil & 4 & $0-$ & 4 \\
\hline Clay & 51 & 4- & 55 \\
\hline Sand & 10 & $55-$ & 65 \\
\hline Clay, red & 85 & $65-$ & 150 \\
\hline Sand, coarse and fine gravel & 52 & $150-$ & 202 \\
\hline Shale, blue & 17 & 202- & 219 \\
\hline Sand, coarse and gravel & 31 & 219- & 250 \\
\hline Shale, gray & 82 & $250-$ & 332 \\
\hline Sand, sandy and shale & 49 & $332-$ & 381 \\
\hline Shale, sandy & 17 & $381-$ & 398 \\
\hline Sand & 21 & 398- & 419 \\
\hline Shale, brown and white & 35 & 419- & 454 \\
\hline Sand and sandy shale & 32 & 454- & 486 \\
\hline Shale and sand streaks & 14 & 486- & 500 \\
\hline Sand and fine gravel & 57 & $500-$ & 557 \\
\hline Shale & 15 & $557-$ & 572 \\
\hline Sand and hard sand & 58 & $572-$ & 630 \\
\hline Sand, hard and shale streaks & 64 & $630-$ & 694 \\
\hline Shale & 26 & 694- & 720 \\
\hline Sand & 32 & $720-$ & 752 \\
\hline Shale & 16 & $752-$ & 768 \\
\hline Sand & 14 & $768-$ & 782 \\
\hline Shale, sandy and sand & 27 & $782-$ & 809 \\
\hline Sand & 26 & 809- & 835 \\
\hline $\begin{array}{l}\text { Shale, hard layers and streaks of } \\
\text { sandy shale }\end{array}$ & 55 & $835-$ & 890 \\
\hline
\end{tabular}


Table 3.--Drillers' logs of selected wells in Fort Bend County, 1969-87--Cont inued

Hell JY-65-19-904--Cont inued

Sand, coarse and hard layers

Sand, hard and coarse

Shale

Sand

Shale, sandy and sand streaks

Shale and sand streaks

Shale and hard sand streaks

Shale

Shale and sandy shale

Sand and sandy shale

Shale, sandy

Sand, fine and shale breaks

Shale

Sand

Shale

Sand and sandy shale

Sand and streaks of shale

Sand, hard

Shale

Sand and sandy shale, rock and shale layers

Shale, blue and sand streaks

Sand, hard and shale

shale, sandy

Sand

Sand, broken

Shale

Well JY-65-19-905

Owner: Fort Bend County Municipal Utility Utility District 25 , Well No. 2

Driller: Water Resources of Texas

\begin{tabular}{lrr}
\hline Description & $\begin{array}{c}\text { Thick- } \\
\text { ness } \\
\text { (feet) }\end{array}$ & $\begin{array}{c}\text { Depth } \\
\text { interval } \\
\text { (feet) }\end{array}$ \\
\hline Unrecorded & 562 & $0-562$ \\
Sand & 94 & $562-656$ \\
Shale & 16 & $656-672$ \\
Sand & 30 & $672-702$ \\
Shale & 42 & $702-744$ \\
Sand & 44 & $744-788$
\end{tabular}

$39 \quad 1,337-1,376$

$5 \quad 1,376-1,381$

$16 \quad 1,381-1,397$

$18 \quad 1,397-1,415$

$42 \quad 1,415-1,457$

$15 \quad 1,457-1,472$

$37 \quad 1,472-1,509$
Well JY-65-19-905--Continued

$\begin{array}{lcc}\text { Shale } & 10 & 788-798 \\ \text { Sand } & 42 & 798-840 \\ \text { Shale } & 16 & 840-856 \\ \text { Sand } & 58 & 856-914 \\ \text { Shale } & 10 & 914-924\end{array}$

Well JY-65-19-906

Owner: Fort Bend County Municipal Utility District 41, Hell No. 1

Driller: Layne-Texas Co.

\begin{tabular}{ccc}
\hline Description & $\begin{array}{c}\text { Thick- } \\
\text { ness } \\
\text { (feet) }\end{array}$ & $\begin{array}{c}\text { Depth } \\
\text { interval } \\
\text { (feet) }\end{array}$ \\
\hline
\end{tabular}

Topsoil

$0-1$

clay

Clay and sandy clay

1. 9

Sand and gravel streaks

9- 11

Sand and clay streaks

$11-41$

Clay

$41-43$

Clay and gravel

43- 48

Sand and gravel

48- 49

Clay

49- 53

53- 55

Sand, gravel and clay streaks

55- 58

clay

$58-61$

Clay and sandy clay

$61-63$

Sand

$63-66$

Clay

$66-76$

Sand

76- 77

clay

$77-79$

Sand and sandy clay streaks

79- 82

Clay

82- 102

Sand

$102-104$

clay

$104-107$

Clay and sandy clay streaks

107-135

Clay and lime streaks

$135-140$

Clay and sandy clay streaks

$140-156$

Gravel, clay and sandy clay

156-159

Sand and gravel

159-187

Clay and gravel streaks

$187-191$

Sand, gravel and lime streaks

191-223

Sand, gravel and clay streaks 
Well JY-65-19-906--Cont inued

Sand and sandy clay

Sand and gravel streaks

Clay and sandy clay

Sand and clay streaks

Sand, gravel, and clay streaks

Clay and lime streaks

Clay

Clay, shale and sand streaks

Sand and gravel

Lime

clay

Clay, sandy and sand streaks

Sand, gravel, and lime

Clay

Clay, sandy and sand streaks

Lime and sandstone

Lime, gravel, sand, and clay

Lime, clay, and sandstone

Sand, gravel and clay streaks

Sand, limestone, gravel and clay

Sand, clay and sandy clay streaks

Clay and lime streaks

Clay, lime and sand streaks

Clay, sandy, clay and lime

Sand, lime and clay streaks

Clay, sandy and lime streaks

Sand, lime and clay streaks

Lime

Clay, sandy with clay and lime streaks

Sand, sandy clay and lime streaks

Clay and lime streaks

Sand and sandstone

Sand, sandstone and clay streaks

clay and sandstone

Sand, sands tone and clay

Clay, sandy clay and lime streaks

Clay, sandstone, sand streaks and gravel
Hell JY-65-19-906--Cont inued

Clay and sandy clay

$8905-913$

clay and lime

21 913- 934

Clay and sandy clay streaks

Clay

Clay and lime streaks

Clay, sandy, lime and clay streaks

clay, lime and shale streaks

Shale and sand streaks

Shale and clay

Sand and shale streaks

Shale with clay and Iime streaks

Clay

Clay, sandy clay, and sand

Sand and sandy clay streaks

sand, sandy clay and clay streaks

Clay and sandy clay

Clay, sandy clay and sand streaks

Clay, lime and shale streaks

Clay, shale, lime and sandy clay streaks

$607-637$

$637-650$

$650-660$

$660-672$

672-681

$681-708$

708-725

$725-768$

$768-770$

2

10

$770-780$

780-786

786-794

794-804

804-827

827-829

829-887

887-896

9

896-905 clay

clay, sandy, clay and sand streaks

Clay

Clay, sandy clay and shale

Clay and lime streaks

Clay, shale, few sandy clay streaks

Clay and lime streaks

clay and shale

Clay and sandy clay

Sand

Shale and clay streaks

Sand and sandy clay streaks Clay, sandy clay, sand and broken
shale

Clay, sandy clay and shale

Clay, sandy, sand and shale

Clay

Clay, tough

Sand and clay streaks $\begin{array}{rr}4 & 934-938 \\ 99 & 988-1,037\end{array}$

$\begin{array}{rr}4 & 934-938 \\ 99 & 988-1,037\end{array}$

$23 \quad 1,037-1,060$

$18 \quad 1,060-1,078$

$421,078-1,120$

$121,120-1,132$

$19 \quad 1,132-1,151$

$11 \quad 1,151-1,162$

$20 \quad 1,162-1,182$

$4 \quad 1,182-1,186$

$31,186-1,189$

4 1,189-1,193

$8 \quad 1,193-1,201$

$16 \quad 1,201-1,217$

$3 \quad 1,217-1,220$

$17 \quad 1,220-1,237$

$11 \quad 1,237-1,248$

1 1,248-1,249

$221,249-1,271$

$8 \quad 1,271-1,279$

5 1,279-1,284

11 1,284-1,295

7 1,295-1,302

$131,302-1,315$

$30 \quad 1,215-1,345$

$21,345-1,347$

$3 \quad 1,347-1,350$

$8 \quad 1,350-1,358$

$10 \quad 1,358-1,368$

$16 \quad 1,368-1,384$

$14 \quad 1,384-1,398$

$351,398-1,433$

$14 \quad 1,433-1,447$

$11 \quad 1,447-1,458$

$32 \quad 1,458-1,490$ 
Well JY-65-19-906--Cont inued

Sand, lime and shale streaks

Lime, hard

Sand, lime and clay streaks

Clay, shale and sandy clay

Clay and shale streaks

Clay, tough

Clay and shale

Lime, hard

Clay and lime streaks

Clay and shale with few lime and sand streaks

$4 \quad 1,635-1,639$

Sand, lime and clay streaks

$261,639-1,665$

Clay, sandy clay and sand streaks

$111,665-1,676$

Clay and sandy clay streaks

Clay, sandy clay and sand streaks

Clay, lime and shale streaks

$91,676-1,685$

$131,685-1,698$

$5 \quad 1,698-1,703$

Clay, sandy clay, sand and lime streaks

Clay and lime streaks

Lime, hard and clay

Clay, sandy clay and lime streaks

Lime, hard and clay

Clay and lime streaks

Well JY-65-19-907

Owner: Fort Bend County Municipal Utility District 2, Well No. 1

Driller: Texas Water Wells, Inc.

\begin{tabular}{lcc}
\hline \multicolumn{1}{c}{ Description } & $\begin{array}{c}\text { Thick- } \\
\text { ness } \\
\text { (feet) }\end{array}$ & $\begin{array}{c}\text { Depth } \\
\text { interval } \\
\text { (feet) }\end{array}$ \\
\hline Clay & 105 & $0-105$ \\
Sand & 173 & $105-278$ \\
Clay & 37 & $278-315$ \\
Sand & 15 & $315-330$ \\
Clay & 70 & $330-400$ \\
Sand & 15 & $400-415$ \\
Clay & 25 & $415-440$ \\
Sand & 72 & $440-512$ \\
Clay with sand streaks & 38 & $512-550$ \\
Sand & 62 & $550-612$ \\
Clay & 6 & $612-618$
\end{tabular}

Well JY-65-19-907--Cont inued

\begin{tabular}{lcc} 
Sand & 10 & $618-628$ \\
Shale & 28 & $628-656$ \\
Sand & 14 & $656-670$ \\
Shale & 8 & $670-678$ \\
Sand & 24 & $678-702$ \\
Shale & 5 & $702-707$ \\
Sand & 10 & $707-717$ \\
Shale & 11 & $717-728$ \\
Sand & 20 & $728-748$ \\
Shale & 14 & $748-762$ \\
Sand & 62 & $762-824$ \\
Shale & 28 & $824-852$ \\
Sand & 10 & $852-862$ \\
Shale & 10 & $862-872$ \\
Sand & 20 & $872-892$ \\
Shale & 20 & $892-912$ \\
\hline
\end{tabular}

Well JY-65-19-909

Owner: Gemstar Homes

Driller: O'Day Drilling Co., Inc.

\begin{tabular}{|c|c|c|}
\hline Description & $\begin{array}{l}\text { Thick- } \\
\text { ness } \\
\text { (feet) }\end{array}$ & $\begin{array}{l}\text { Depth } \\
\text { interval } \\
\text { (feet) }\end{array}$ \\
\hline Topsoil & 3 & $0-3$ \\
\hline Clay & 28 & 3- 31 \\
\hline Sand & 42 & $31-73$ \\
\hline Clay & 32 & $73-105$ \\
\hline Sand & 99 & $105-204$ \\
\hline Clay & 14 & $204-218$ \\
\hline Sand & 39 & $218-257$ \\
\hline Clay & 23 & $257-280$ \\
\hline Sand & 65 & $280-345$ \\
\hline Clay & 35 & $345-380$ \\
\hline Sand & 20 & $380-400$ \\
\hline Clay & 3 & $400-403$ \\
\hline Sand & 35 & $403-438$ \\
\hline Clay & 32 & $438-470$ \\
\hline Sand & 6 & $470-476$ \\
\hline Clay & 11 & $476-487$ \\
\hline
\end{tabular}


Well JY-65-19-909--Cont inued

$\begin{array}{lcc}\text { Sand } & 58 & 487-545 \\ \text { Clay } & 4 & 545-549\end{array}$

Well JY-65-19-910

Owner: Fort Bend County Municipal Utility District, Well No. 2

Driller: Layne-Western Co., Inc.

\begin{tabular}{|c|c|c|c|c|c|}
\hline Description & $\begin{array}{l}\text { Thick- } \\
\text { ness } \\
\text { (feet) }\end{array}$ & $\begin{array}{c}\text { Depth } \\
\text { interval } \\
\text { (feet) }\end{array}$ & $\begin{array}{l}\text { Clay and sand streaks } \\
\text { Sand }\end{array}$ & $\begin{array}{l}82 \\
10\end{array}$ & $\begin{array}{r}33-115 \\
115-125\end{array}$ \\
\hline Surface & 33 & $0-33$ & Shale, hard & 31 & $125-156$ \\
\hline Unrecorded & 23. & $33-56$ & Shale, sandy and sand streaks & 34 & $156-190$ \\
\hline Sand and clay & 48 & $56-104$ & Shale and hard streaks & 30 & $190-220$ \\
\hline Sand & 6 & $104-200$ & Shale & 8 & $220-228$ \\
\hline Clay and sand streaks & 121 & $200-321$ & Sand & 62 & $228-290$ \\
\hline Sand and rock & 35 & $321-356$ & Shale & 20 & $290-310$ \\
\hline Clay & 49 & $356-405$ & Sand & 29 & $310-339$ \\
\hline Sand & 15 & $405-420$ & Shale & 8 & $339-347$ \\
\hline Clay & 7 & $420-427$ & Sand & 23 & $347-370$ \\
\hline Sand & 36 & $427-463$ & Shale & 8 & $370-378$ \\
\hline Clay & 2 & $463-465$ & Sand & 13 & $378-391$ \\
\hline Sand & 12 & $465-477$ & Shale & 9 & $391-400$ \\
\hline Clay, hard & 23 & $477-500$ & Sand and shale streaks & 30 & $400-430$ \\
\hline Sand and rock & 27 & $500-527$ & Shale & 4 & $430-434$ \\
\hline Clay, hard & 7 & $527-534$ & Sand and gravel & 27 & $434-461$ \\
\hline Sand & 140 & $534-674$ & Shale & 14 & $461-475$ \\
\hline Clay & 10 & $674-684$ & Shale & 29 & $475-504$ \\
\hline Sand and broken rock & 110 & $684-794$ & Sand and gravel & 70 & $504-574$ \\
\hline Sand and clay & 100 & $794-894$ & Shale & 14 & $574-588$ \\
\hline Sand streaks & 13 & $894-907$ & Sand and shale breaks & 20 & $588-608$ \\
\hline Rock & 2 & $907-909$ & Shale & 10 & $608-618$ \\
\hline Rock and broken sand & 27 & $909-936$ & Sand and sandy shale & 31 & $618-649$ \\
\hline Clay and sand & 23 & $936-959$ & Rock and hard shale & 5 & $649-654$ \\
\hline Rock & 13 & $959-972$ & Sand and sandy shale & 15 & $654-669$ \\
\hline \multirow[t]{7}{*}{ Clay } & 22 & $972-994$ & Shale & 13 & $669-682$ \\
\hline & & & Sand and shale breaks & 56 & $682-738$ \\
\hline & & & Shale, sandy and sand & 16 & $738-754$ \\
\hline & & & Shale & 16 & $754-770$ \\
\hline & & & Sand and shale streaks & 27 & $770-797$ \\
\hline & & & Shale and sandy shale & 18 & $797-815$ \\
\hline & & & Sand & 37 & $815-852$ \\
\hline
\end{tabular}

Well JY-65-20-708

Owner: Fort Bend County Water Control and Improvement District 2

Driller: Layne-Texas Co.

\begin{tabular}{lcc}
\hline Description & $\begin{array}{c}\text { Thick- } \\
\text { ness } \\
\text { (feet) }\end{array}$ & $\begin{array}{c}\text { Depth } \\
\text { interval } \\
\text { (feet) }\end{array}$ \\
\hline
\end{tabular}

Clay and sandy clay $0-33$ -115 $-125$

$5-549$ 
Well JY-65-20-708--Cont inued

$\begin{array}{lrr}\text { Sand and shale streaks } & 46 & 852-898 \\ \text { Sand } & 53 & 898-951 \\ \text { Shale } & 7 & 951-958 \\ \text { Sand and gravel } & 120 & 958-1,078 \\ \text { Sand and gravel and shale } & 46 & 1,078-1,124 \\ \text { Shale, sandy and sand } & 18 & 1,124-1,142 \\ \text { Shale } & 104 & 1,142-1,246 \\ \text { Shale, sandy and sand streaks } & 33 & 1,246-1,279 \\ \text { Shale } & 78 & 1,279-1,357 \\ \text { Sand, shale and sand streaks } & 33 & 1,357-1,390 \\ \text { Sand, broken } & 16 & 1,390-1,406 \\ \text { Shale, sandy and sand } & 18 & 1,406-1,424 \\ \text { Sand and sandy shale } & 21 & 1,424-1,445 \\ \text { Shale } & 25 & 1,445-1,470 \\ \text { Sand and shale streaks } & 32 & 1,470-1,502 \\ \text { Shale } & 22 & 1,502-1,524 \\ \text { Sand } & 99 & 1,524-1,623 \\ \text { Shale } & 4 & 1,623-1,627 \\ \text { Sand } & 24 & 1,627-1,658 \\ \text { Shale and sand streaks } & 18 & 1,682-1,700 \\ \text { Shale } & 31-1,682 \\ & & \end{array}$

Well JY-65-20-709

Owner: The Meadows Municipal Utility

District, Well No. 2

Driller: Layne-Texas Co.

\begin{tabular}{lrrr}
\hline \multicolumn{1}{c}{ Description } & $\begin{array}{c}\text { Thick- } \\
\text { ness } \\
\text { (feet) }\end{array}$ & $\begin{array}{c}\text { Depth } \\
\text { interval } \\
\text { (feet) }\end{array}$ \\
\hline Topsoil & 5 & $0-$ & 5 \\
Clay & 20 & $5-$ & 25 \\
Sand & 125 & $25-$ & 150 \\
Shale and few sand streaks & 95 & $150-$ & 245 \\
Sand and shale streaks & 184 & $245-$ & 429 \\
Shale & 21 & $429-$ & 450 \\
Sand and shale streaks & 80 & $450-$ & 530 \\
Sand and fine gravel & 103 & $530-$ & 633 \\
Shale and sand streaks & 47 & $633-$ & 680 \\
Sand and few shale streaks & 100 & $680-$ & 780 \\
Shale & 20 & $780-$ & 800 \\
Sand & 23 & $800-$ & 823
\end{tabular}

Well JY-65-20-709--Cont inued

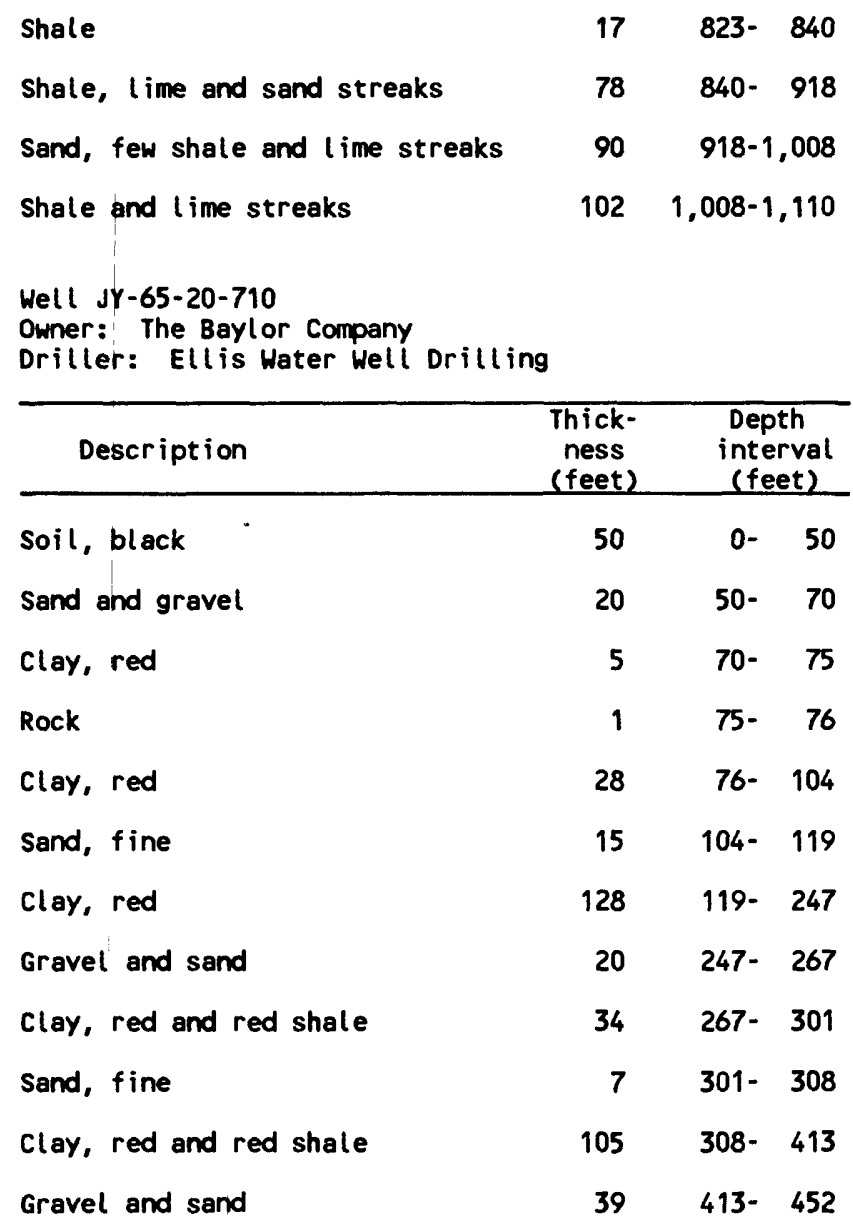

Well dY-65-20-711

Owner: City of Sugarland

Driller: Layne-Texas Co.

\begin{tabular}{lrrr}
\hline \multicolumn{1}{c}{ Description } & $\begin{array}{c}\text { Thick- } \\
\text { ness } \\
\text { (feet) }\end{array}$ & $\begin{array}{c}\text { Depth } \\
\text { interval } \\
\text { (feet) }\end{array}$ \\
\hline Clay & 17 & $0-$ & 17 \\
Sand & 24 & $17-$ & 41 \\
Sand and gravel & 31 & $41-$ & 72 \\
Clay & 24 & $72-$ & 96 \\
Sand and clay streaks & 38 & $96-$ & 134 \\
Clay & 18 & $134-$ & 152 \\
Sand and gravel & 59 & $152-$ & 211 \\
Shale & 9 & $211-$ & 220 \\
Sand & 59 & $220-$ & 279 \\
Shale & 20 & $279-$ & 299 \\
Shale, sandy & 5 & $299-$ & 304 \\
Shale & 41 & $304-$ & 345
\end{tabular}


Well JY-65-20-711--Continued

Shale, sandy and sand

Shale

Sand and sandy shale

Shale

Shale and sandy shale

Sand and sandy shale

Shale

Sand and sandy shale

Shale

Sand

Shale

Sand and shale

Sand and shale breaks

Shale and sand streaks

Sand and sandy shale

Shale

Sand and shale streaks

Shale

Sand and sandy shale

Shale

Sand and sandy shale

Shale

Shale, sandy

Shale

Sand and sandy shale

Shale

Sand and sandy shale

Shale

Sand and shale

Sand

Shale

Sand and sandy shale

Shale and sandy shale

Sand and shale

Shale

Sand and sandy shale

Shale and sand streaks

Sand and shale streaks
16 368- 384

22 384- 406

$24 \quad 406-430$

$25 \quad 430-455$

$20 \quad 455-475$

$26 \quad 475-501$

$65 \quad 501-566$

$16 \quad 566-582$

$18 \quad 582-600$

$28 \quad 600-628$

14 628- 642

22 642- 664

18 664- 682

$19 \quad 682-701$

$14 \quad 701-715$

$30 \quad 715-745$

$41 \quad 745-786$

$12 \quad 786-798$

$18 \quad 798-816$

42

816- 858

858- 868

15

868- 883

883- 903

903- 941

38

11

19

941- 952

19

952- 971

4 971- 975

$45975-1,020$

$301,020-1,050$

$88 \quad 1,050-1,138$

$15 \quad 1,138-1,153$

$1221,153-1,275$

$441,275-1,319$

$11 \quad 1,319-1,330$

$971,330-1,427$

$44 \quad 1,427-1,471$

$29 \quad 1,471-1,500$
Well JY-65-20-711--Continued

Shale

$61,500-1,506$

Sand and shale streaks

$40 \quad 1,506-1,546$

Shale, sandy and sand

$40 \quad 1,546-1,586$

Shale, sandy and shale

$37 \quad 1,586-1,623$

Sand and sandy shale

$331,623-1,656$

Shale

$18 \quad 1,656-1,674$

Shale, hard

$81,674-1,682$

Shale

$18 \quad 1,682-1,700$

Well JY-65-20-804

Owner: Weatherford Farm and Greenhouse, Inc.

Driller: Bryan Drilling Co.

\begin{tabular}{lrrr}
\hline \multicolumn{1}{c}{ Description } & $\begin{array}{c}\text { Thick- } \\
\text { ness } \\
\text { (feet) }\end{array}$ & $\begin{array}{c}\text { Depth } \\
\text { interval } \\
\text { (feet) }\end{array}$ \\
\hline Surface & 4 & $0-$ & 4 \\
Clay and sand & 75 & $4-$ & 79 \\
Sand & 21 & $79-$ & 100 \\
Shale & 105 & $100-$ & 205 \\
Sand & 4 & $205-$ & 209 \\
Clay & 20 & $209-$ & 229 \\
Sand & - & $229-$ & - \\
Clay & - & $229-$ & 280 \\
Sand & 24 & $280-$ & 304 \\
Clay & 42 & $304-$ & 346 \\
Sand & 31 & $346-377$
\end{tabular}

Well JY-65-20-805

Owner: Texas Instruments, Inc.

Driller: Layne-Texas Co.

\begin{tabular}{lccr}
\hline \multicolumn{1}{c}{ Description } & $\begin{array}{c}\text { Thick- } \\
\text { ness } \\
\text { (feet) }\end{array}$ & $\begin{array}{c}\text { Depth } \\
\text { interval } \\
\text { (feet) }\end{array}$ \\
\hline Topsoil and clay & 15 & $0-$ & 15 \\
Sand, fine, red & 15 & $15-$ & 30 \\
Clay, red & 55 & $30-$ & 85 \\
Sand and sandy clay & 15 & $85-100$ \\
Sand and hard streaks & 10 & $100-110$ \\
Clay, sandy and sand & 28 & $110-138$ \\
Clay, sticky, red & 27 & $138-165$ \\
Clay, sandy & 29 & $165-194$ \\
Clay, sandy and sand & 10 & $194-$ & 204
\end{tabular}


Well JY-65-20-805--Cont inued

Clay, sandy and clay

Sand and clay streaks

streaks of sand and shale

Sand

streaks of sand and shale

Sand and fine gravel

Shale

Sand, fine

Shale

Sand, coarse and gravel

Shale and sand streaks

Shale, sandy

Sand

Sand and streaks, shale

Shale and layers, sand

Sand, broken

Shale and sandy shale

Sand and streaks, shale

Sand and shate, sandy

Sand, broken

Shale and shate sandy

Sand and shale sandy

Shale

Sand and shale sandy

Shale

sand, broken

Shale

Sand and shale breaks

Shale

Shale and sand breaks

Sand

Shale

\begin{tabular}{|c|c|c|}
\hline$? 1$ & $204-$ & 225 \\
\hline 68 & 225- & 293 \\
\hline & 293- & 329 \\
\hline & 329- & 379 \\
\hline & 379- & 422 \\
\hline & 422- & 450 \\
\hline & 450- & 479 \\
\hline & 479- & 497 \\
\hline & 497- & 512 \\
\hline & 512- & 600 \\
\hline & $600-$ & 625 \\
\hline & $625-$ & 644 \\
\hline & $644-$ & 668 \\
\hline & 668- & 689 \\
\hline & 689- & 700 \\
\hline & $700-$ & 735 \\
\hline & $735-$ & 749 \\
\hline & 649- & 764 \\
\hline & $764-$ & 780 \\
\hline & $780-$ & 793 \\
\hline & 793- & 834 \\
\hline 9 & 834- & 853 \\
\hline & 853- & 868 \\
\hline 14 & 868- & 882 \\
\hline 8 & 882- & 890 \\
\hline & 890- & 911 \\
\hline & 911 - & 928 \\
\hline & 928- & 949 \\
\hline 2 & 949- & 951 \\
\hline & 951- & 980 \\
\hline & $980-1$ & .004 \\
\hline & 1.004 & \\
\hline
\end{tabular}

Well JY-65-20-806

Owner: The Meadows Municipal Utility District, Well No. 1

Driller: Layne-Texas Co.

\begin{tabular}{|c|c|c|c|}
\hline Description & $\begin{array}{l}\text { Thick- } \\
\text { ness } \\
\text { (feet) }\end{array}$ & $\begin{array}{l}\text { Dep } \\
\text { inte } \\
\text { sfe }\end{array}$ & $\begin{array}{l}\text { th } \\
\text { rval } \\
\text { et) }\end{array}$ \\
\hline Topsoil & 4 & $0-$ & 4 \\
\hline $\mathrm{Cl}$ ay and sand streak & 114 & 4- & 118 \\
\hline Sand & 4 & $118-$ & 122 \\
\hline Shale & 39 & $122-$ & 161 \\
\hline Sand & 126 & $161-$ & 287 \\
\hline Shale & 21 & $287-$ & 308 \\
\hline Sand & 38 & $308-$ & 346 \\
\hline Shale ans sand streaks & 40 & $346-$ & 386 \\
\hline Sand and shale & 40 & $386-$ & 426 \\
\hline Sand & 22 & 426- & 448 \\
\hline Sand and sandy shale & 21 & 448- & 469 \\
\hline Shale & 31 & 469- & 500 \\
\hline Sand & 12 & $500-$ & 512 \\
\hline Shale, sandy & 10 & $512-$ & 522 \\
\hline Sand and shale streaks & 34 & $522-$ & 556 \\
\hline Shale & 7 & $556-$ & 563 \\
\hline Sand and shale streaks & 24 & $563-$ & 587 \\
\hline Sand and gravel & 55 & $587-$ & 642 \\
\hline Shale, hard and sandy & 41 & $642-$ & 683 \\
\hline Sand and shale, sandy & 18 & $683-$ & 701 \\
\hline Shale & 33 & $701-$ & 734 \\
\hline Shale, sandy and sand & 18 & $734-$ & 752 \\
\hline Sand and shale streaks & 20 & $752-$ & 772 \\
\hline Shale & 37 & $772-$ & 809 \\
\hline Sand & 27 & 809- & 836 \\
\hline Shale & 7 & $836-$ & 843 \\
\hline Sand & 7 & 843- & 850 \\
\hline Sand & 100 & 850- & 950 \\
\hline Shale & 16 & $950-$ & 966 \\
\hline Sand & 58 & \multicolumn{2}{|c|}{$966-1,024$} \\
\hline Shale & 48 & \multicolumn{2}{|c|}{$1,024-1,072$} \\
\hline Sand & 15 & \multicolumn{2}{|c|}{$1,072-1,087$} \\
\hline Shale & 13 & \multicolumn{2}{|c|}{$1,087-1,100$} \\
\hline
\end{tabular}


Well JY-65-20-914

Owner: Fort Bend County Hater Control and Improvement District 2

Driller: Layne-Texas Co.

\begin{tabular}{|c|c|c|}
\hline Description & $\begin{array}{l}\text { Thick- } \\
\text { ness } \\
\text { (feet) }\end{array}$ & $\begin{array}{l}\text { Depth } \\
\text { interval } \\
\text { (feet) }\end{array}$ \\
\hline Topsoil & 2 & $0-$ \\
\hline Sand and clay streaks & 11 & $2-$ \\
\hline Clay, brown & 6 & 13- \\
\hline Clay, sandy gray & 4 & $19-$ \\
\hline Clay, brown and sand streaks & 19 & 23- \\
\hline Clay, brown and gray & 8 & 42- \\
\hline $\begin{array}{l}\text { Clay, brown and gray with } \\
\text { chalk streaks }\end{array}$ & 10 & $50-$ \\
\hline Clay, red with chalk streaks & 108 & $60-168$ \\
\hline Clay, gray and blue & 37 & $168-205$ \\
\hline Sand, coarse and shale streaks & 41 & $205-246$ \\
\hline Shale, sandy & 64 & $246-310$ \\
\hline Sand and shale streaks & 10 & $310-320$ \\
\hline Clay, gray and sand streaks & 5 & $320-325$ \\
\hline Shale, brown & 56 & $325-\quad 381$ \\
\hline Sand and shale streaks & 25 & $381-406$ \\
\hline Shale, soft brown and sand streaks & 90 & 406- 496 \\
\hline Sand and shale streaks & 114 & 496- 610 \\
\hline Shale, white and sand streaks & 47 & $610-657$ \\
\hline Clay, brown and sand streaks & 89 & $657-746$ \\
\hline Shale, dry, brown with sand streaks & 75 & $746-821$ \\
\hline Clay, soft gray & 15 & $821-836$ \\
\hline $\begin{array}{l}\text { Shale, brown-to-gray wi th } \\
\text { sand streaks }\end{array}$ & 109 & 836- 945 \\
\hline Sand and shale streaks & 35 & $945-980$ \\
\hline Shale and sand streaks & 30 & $980-1,010$ \\
\hline Shale, sandy & 15 & $1,010-1,025$ \\
\hline Sand and shale streaks & 34 & $1,025-1,059$ \\
\hline Shale and sand streaks & 14 & $1,059-1,073$ \\
\hline Sand and shale streaks & 12 & $1,073-1,085$ \\
\hline Shale and sand streaks & 160 & $1,085-1,245$ \\
\hline Shale, sandy & 51 & $1,245-1,296$ \\
\hline Sand & 17 & $1,296-1,313$ \\
\hline Shale and sand streaks & 46 & $1,313-1,359$ \\
\hline Sand and shale streaks & 20 & $1,359-1,379$ \\
\hline
\end{tabular}

Well JY-65-20-914--Cont inued

Sand

$$
\begin{array}{rr}
40 & 1,379-1,419 \\
6 & 1,419-1,425 \\
50 & 1,425-1,475 \\
41 & 1,475-1,516 \\
22 & 1,516-1,538 \\
5 & 1,538-1,543 \\
17 & 1,543-1,560 \\
16 & 1,560-1,576 \\
24 & 1,576-1,600 \\
27 & 1,600-1,627 \\
51 & 1,627-1,678 \\
22 & 1,678-1,700
\end{array}
$$

Sand and shale streaks

Shale and sand streaks

Sand and shale streaks

\begin{tabular}{|c|c|c|c|}
\hline Description & $\begin{array}{c}\text { Thick- } \\
\text { ness } \\
\text { (feet) }\end{array}$ & $\begin{array}{r}\text { Dep } \\
\text { inte } \\
\text { sfe }\end{array}$ & $\begin{array}{l}\text { th } \\
\text { erval } \\
\text { et) }\end{array}$ \\
\hline Surface clay & 30 & $0-$ & 30 \\
\hline Sand and gravel & 24 & $30-$ & 54 \\
\hline Clay & 5 & $54-$ & 59 \\
\hline Sand and rock & 47 & $59-$ & 106 \\
\hline Gravel & 7 & $106-$ & 113 \\
\hline Sand, rock, and gravel & 142 & $113-$ & 255 \\
\hline Clay & 26 & $255-$ & 281 \\
\hline Sand & 43 & 281- & 324 \\
\hline Clay & 9 & 324- & 333 \\
\hline $\mathrm{Cl}$ ay and broken sand & 6 & 333- & 339 \\
\hline Clay & 7 & 339- & 346 \\
\hline Sand and rock & 50 & $346-$ & 396 \\
\hline Clay & 36 & 396- & 432 \\
\hline Sand and rock & 10 & 432- & 442 \\
\hline Clay & 10 & $442-$ & 452 \\
\hline Sand and rock & 10 & 452- & 462 \\
\hline Clay & 10 & $462-$ & 472 \\
\hline Sand and rock & 6 & $472-$ & 478 \\
\hline Shale, hard & 16 & $478-$ & 494 \\
\hline Sand and rock & 7 & 494- & 501 \\
\hline Shale & 21 & $501-$ & 522 \\
\hline
\end{tabular}

Shale and sand streaks

Sand

Sand and shale streaks

Shale and sand streaks

Sand and shale layers

Shale and sand streaks

Sand and shale streaks

Shale

Hell JY-65-25-407

Owner: Heirs of IVy M. Morrison

Driller: Layne-Western Co., Inc. 
Table 3. --prillers' logs of selected wells in Fort Bend County, 1969-87--Cont inued

Well JY-65-25-407--Cont inued

Sand and rock

Shale

Sand and rock

Shale, hard

Sand and rock

Shale

Sand and rock

Shale

Sand and rock

Clay

Sand and rock

Clay

Sand and rock

Clay

Sand, hard and rock

Clay

Sand and rock

Clay

Sand and rock

Clay

Well JY-65-25-505

Owner: Greenwald and Banfield

Driller: Katy Drilling, Inc.

\begin{tabular}{lrr}
\hline \multicolumn{1}{c}{ Description } & $\begin{array}{c}\text { Thick- } \\
\text { ness } \\
\text { (feet) }\end{array}$ & $\begin{array}{c}\text { Depth } \\
\text { interval } \\
\text { (feet) }\end{array}$ \\
\hline Clay, surface & 27 & $0-27$ \\
Sand & 29 & $27-56$ \\
Clay & 7 & $56-63$ \\
Sand & 22 & $63-85$ \\
Clay & 11 & $85-96$ \\
Sand and gravel & 19 & $96-115$ \\
Clay & 9 & $115-124$ \\
Sand and gravel & 92 & $124-216$ \\
Clay & 7 & $216-223$ \\
Sand and gravel & 65 & $223-288$ \\
Clay & 7 & $288-295$ \\
Gravel & 5 & $295-300$ \\
Clay & 26 & $300-326$
\end{tabular}

Hell JY-65-25-505--Cont inued

\begin{tabular}{|c|c|c|}
\hline Sand & 15 & $326-341$ \\
\hline Clay & 3 & $341-344$ \\
\hline Sand & 30 & $344-374$ \\
\hline Clay & 9 & $374-383$ \\
\hline Sand and rock & 59 & $383-442$ \\
\hline Clay & 22 & $442-464$ \\
\hline Sand and rock & 22 & $464-486$ \\
\hline Clay & 82 & $486-568$ \\
\hline Sand and rock & 12 & $568-580$ \\
\hline Clay & 11 & $580-591$ \\
\hline Sand and rock & 55 & $591-646$ \\
\hline Clay & 8 & $646-654$ \\
\hline Sand and rock & 24 & $654-678$ \\
\hline Clay & 11 & $678-689$ \\
\hline Sand and rock & 49 & $689-738$ \\
\hline Clay I & 27 & $738-765$ \\
\hline Sand & 28 & $765-793$ \\
\hline Clay & 24 & $793-817$ \\
\hline Sand & 13 & $817-830$ \\
\hline Clay & 5 & $830-835$ \\
\hline Sand & 5 & $835-840$ \\
\hline Clay & 52 & $840-892$ \\
\hline Sand & 11 & $892-903$ \\
\hline
\end{tabular}

Well JY-65-25-506

Owner: John Moore

Driller: Katy Drilling, Inc.

\begin{tabular}{lcr}
\hline \multicolumn{1}{c}{ Description } & $\begin{array}{c}\text { Thick- } \\
\text { ness } \\
\text { (feet) }\end{array}$ & $\begin{array}{c}\text { Depth } \\
\text { interval } \\
\text { (feet) }\end{array}$ \\
\hline Clay, surface & 8 & $0-8$ \\
Sand and clay breaks & 31 & $8-39$ \\
Gravel and sand & 42 & $39-81$ \\
Clay & 27 & $81-108$ \\
Sand and gravel & 53 & $108-161$ \\
Clay & 17 & $161-178$ \\
Sand and gravel & 86 & $178-264$ \\
Clay & 27 & $264-291$ \\
Sand and rock & 13 & $291-304$ \\
Clay & 39 & $304-343$
\end{tabular}


Well JY-65-25-506--Continued

Sand and rock

Clay

Sand and rock

Clay

Sand and rock

Clay

Sand and rock

Clay

Sand and rock

Shale

Sand and rock

Clay

Sand and rock

Clay

Sand

Clay

Sand

Clay

Send

Clay

Sand

Sand

Hell JY-65-25-605

Owner: Heirs of IVy M. Morrison

Driller: Layne-Western Co., Inc.

\begin{tabular}{lcr}
\hline \multicolumn{1}{c}{ Description } & $\begin{array}{c}\text { Thick- } \\
\text { ness } \\
\text { (feet) }\end{array}$ & $\begin{array}{c}\text { Depth } \\
\text { interval } \\
\text { (feet) }\end{array}$ \\
\hline Clay, surface & 19 & $0-19$ \\
Sand & 6 & $19-25$ \\
Clay & 4 & $25-29$ \\
Sand, rock, and gravel & 55 & $29-84$ \\
Clay & 52 & $84-36$ \\
Sand and gravel & 75 & $136-211$ \\
Clay & 4 & $211-215$ \\
Sand and rock & 34 & $215-249$ \\
Shale & 1 & $249-250$ \\
Sand and rock & 45 & $250-295$ \\
Shale & 5 & $295-300$
\end{tabular}

85

513-598
Hell JY-65-25-605--Continued

Sand and rock

$\begin{array}{rr}7 & 300-307 \\ 21 & 307-328 \\ 2 & 328-330 \\ 5 & 330-335 \\ 11 & 335-346 \\ 3 & 346-349 \\ 33 & 349-382 \\ 3 & 382-385\end{array}$

Shale

Sand and rock

Shale

Sand and rock

Shale, sandy

Sand and rock

Clay

385-405

Shale

20

Sand and rock

405-456

Clay

456-469

Sand and rock

469-505

Clay

$505-563$

Sand and rock

563-599

clay

599-613

Sand and rock

$613-627$

Clay

$627-651$

Sand and rock

651-691

Shale and rock

691-707

Sand and rock

707-749

Clay

749-777

Sand and rock

$777-807$

clay, bottom

93

807-900

Well JY-65-25-606

Owner: Heirs of IVy M. Morrison

Driller: Layne-Western Co., Inc.

\begin{tabular}{lcc}
\hline Description & $\begin{array}{c}\text { Thick- } \\
\text { ness } \\
\text { (feet) }\end{array}$ & $\begin{array}{c}\text { Depth } \\
\text { interval } \\
\text { (feet) }\end{array}$ \\
\hline Clay, surface & 35 & $0-35$ \\
Sand and gravel & 177 & $35-212$ \\
Clay & 9 & $212-221$ \\
Sand and gravel & 15 & $221-236$ \\
Clay & 20 & $236-256$ \\
Sand and gravel & 14 & $256-270$ \\
Clay & 11 & $270-281$ \\
Sand and gravel & 33 & $281-314$ \\
Clay & 23 & $314-337$ \\
Sand and rock & 5 & $337-342$
\end{tabular}


Well JY-65-25-606--Cont inued

\begin{tabular}{|c|c|c|}
\hline Clay & 5 & $342-$ \\
\hline Sand and rock & 57 & $347-$ \\
\hline Clay & 11 & 404- \\
\hline Sand and rock & 11 & 415- \\
\hline Clay & 10 & 426- \\
\hline Sand and rock & 32 & 436- \\
\hline Clay & 21 & $468-$ \\
\hline Sand and rock & 13 & 498- \\
\hline Clay & 27 & $502-$ \\
\hline Sand and rock & 14 & $529-$ \\
\hline Shale & 19 & $543-$ \\
\hline Sand & 3 & $562-$ \\
\hline Shale & 3 & $565-$ \\
\hline Sand and rock & 3 & $568-$ \\
\hline Shale & 17 & $571-$ \\
\hline Sand and rock & 13 & $588-$ \\
\hline Clay & 54 & $601-$ \\
\hline Sand and rock & 44 & $655-$ \\
\hline Clay & 16 & $699-$ \\
\hline Sand and rock & 43 & $715-$ \\
\hline Shale & 26 & $758-$ \\
\hline Sand and rock & 53 & $784-$ \\
\hline Clay & 16 & $837-$ \\
\hline Sand and rock & 59 & 853- \\
\hline Shale & 58 & 912- \\
\hline Sand & 23 & 970- \\
\hline Shale & 11 & $993-1$ \\
\hline
\end{tabular}

Well JY-65-25-607

Owner: Murphy Manufacturing Co.

Driller: Robinson Water Well Service

\begin{tabular}{lrrr}
\hline Description & $\begin{array}{c}\text { Thick- } \\
\text { ness } \\
\text { (feet) }\end{array}$ & $\begin{array}{c}\text { Depth } \\
\text { interval } \\
\text { (feet) }\end{array}$ \\
\hline Clay & 14 & $0-$ & 14 \\
Sand & 189 & $14-$ & 203 \\
Clay & 44 & $203-$ & 247 \\
Sand & 143 & $247-390$
\end{tabular}

Well JY-65-25-909

Owner: Hilford Hopmann

Driller: Katy Drilling, Inc.

\begin{tabular}{lrr}
\hline \multicolumn{1}{c}{ Description } & $\begin{array}{c}\text { Thick- } \\
\text { ness } \\
\text { (feet) }\end{array}$ & $\begin{array}{c}\text { Depth } \\
\text { interval } \\
\text { (feet) }\end{array}$ \\
\hline Surface & 30 & $0-30$ \\
Quicksand, fine & 24 & $30-54$ \\
Sand & 16 & $54-70$ \\
Sand and gravel & 83 & $70-153$ \\
Clay $\quad 12$ & $153-165$ \\
Sand and gravel & 18 & $165-183$ \\
Clay & 12 & $183-195$ \\
Sand & 34 & $195-229$ \\
Clay & 16 & $229-245$ \\
Sand & 114 & $245-359$ \\
Clay & 6 & $359-365$ \\
Sand, lime and rock & 108 & $365-473$ \\
Clay & 6 & $473-479$ \\
Sand, lime and rock with small clay \\
streaks
\end{tabular}

Well JY-65-26-104

Owner: Frito-Lay, Inc.

Driller: Critendon Drilling Service, Inc.

\begin{tabular}{lcc}
\hline \multicolumn{1}{c}{ Description } & $\begin{array}{c}\text { Thick- } \\
\text { ness } \\
\text { (feet) }\end{array}$ & $\begin{array}{c}\text { Depth } \\
\text { interval } \\
\text { (feet) }\end{array}$ \\
\hline Topsoil & 1 & $0-1$ \\
Clay, black & 7 & $1-8$ \\
Clay, brown and gray & 17 & $8-25$ \\
Clay and lime & 9 & $25-34$ \\
Sand and gravel & 92 & $34-126$ \\
Clay & 2 & $126-128$ \\
Clay, sandy and sand streaks & 59 & $128-187$ \\
Clay, blue and brown & 18 & $187-205$ \\
& & \\
Well Jy-65-26-105 \\
Owner: Frito-Lay, Inc. \\
Driller: Critendon Drilling Service, Inc. \\
\hline Dlay, brown
\end{tabular}


Well JY-65-26-105--Cont inued

Sand and sandy breaks

Sand and gravel

Sand and clay

Sand, coarse, white

clay, yellow and blue

Sand and gravel

clay, blue and brown

Sand, medium and coarse

clay, brown and blue

Sand, medium, white

clay, blue and brown

Sand and gravel

clay, blue and brown

Well JY-65-26-407

Owner: Frito-Lay, Inc.

Driller: Critendon Drilling Service, Inc.

\begin{tabular}{lcr}
\hline \multicolumn{1}{c}{ Description } & $\begin{array}{c}\text { Thick- } \\
\text { ness } \\
\text { (feet) }\end{array}$ & $\begin{array}{c}\text { Depth } \\
\text { interval } \\
\text { (feet) }\end{array}$ \\
\hline Sand & 3 & $0-3$ \\
Clay, black-to-gray & 6 & $3-9$ \\
Clay, yellowish gray & 1 & $9-10$ \\
Clay, brown & 24 & $10-34$ \\
Sand, brown & 103 & $34-137$ \\
Sand, coarse, gray & 17 & $137-154$ \\
Clay, brown and blue & 19 & $154-173$ \\
Sand, brown, coarse & 43 & $173-216$ \\
Clay, brown and gray & 33 & $216-249$ \\
Sand and gravel & 26 & $249-275$ \\
Clay, blue and brown & 12 & $275-287$ \\
Sand streaks & 3 & $287-290$ \\
Clay, brown and gray wi th lime & 19 & $290-309$ \\
Sand and gravel & 40 & $309-349$ \\
Shale, brown and blue & 1 & $349-350$
\end{tabular}

Hell JY-65-26-408

Owner: Frito-Lay, Inc.

Driller: Critendon Drilling Service, Inc.

\begin{tabular}{lcc}
\hline \multicolumn{1}{c}{ Description } & $\begin{array}{c}\text { Thick- } \\
\text { ness } \\
\text { (feet) }\end{array}$ & $\begin{array}{c}\text { Depth } \\
\text { interval } \\
\text { (feet) }\end{array}$ \\
\hline Sand & 3 & $0-3$ \\
Clay, black-to-gray & 6 & $3-9$ \\
Clay, yel lowish gray & 1 & $9-10$ \\
Clay, brown & 24 & $10-34$ \\
Sand, brown & 103 & $34-137$ \\
Sand, coarse, gray & 17 & $137-154$ \\
Clay, brown and blue & 19 & $154-173$ \\
Sand, brown, coarse & 43 & $173-216$ \\
Clay, brown and gray & 33 & $216-249$ \\
Sand, medium and gravel & 26 & $249-275$ \\
Clay, blue and brown & 12 & $275-287$ \\
Sand streaks & 3 & $287-290$ \\
Clay, brown and gray with lime & 19 & $290-309$ \\
Sand and gravel & 40 & $309-349$ \\
Shale, brown and blue & 1 & $349-350$ \\
& & \\
& &
\end{tabular}

Well JY-65-26-612

Owner: City of Richmond

Driller: Layne-Western Co., Inc.

\begin{tabular}{lrr}
\hline \multicolumn{1}{c}{ Description } & $\begin{array}{c}\text { Thick- } \\
\text { ness } \\
\text { (feet) }\end{array}$ & $\begin{array}{c}\text { Depth } \\
\text { interval } \\
\text { (feet) }\end{array}$ \\
\hline Clay & 20 & $0-20$ \\
Sand & 4 & $20-24$ \\
Clay & 16 & $24-40$ \\
Sand and gravel & 16 & $40-56$ \\
Clay & 6 & $56-62$ \\
Gravel & 9 & $62-71$ \\
Clay & 23 & $71-94$ \\
Sand and gravel & 10 & $94-104$ \\
Clay & 40 & $104-144$ \\
Sand and gravel & 45 & $144-189$ \\
Clay & 5 & $189-194$ \\
Sand and gravel & 114 & $194-308$ \\
Shale & 4 & $308-312$ \\
Sand and gravel & 6 & $312-318$ \\
Shale & 13 & $318-331$
\end{tabular}


Hell JY-65-26-612---Continued

Gravel and sand

Clay

Sand, gravel, and rock

Shale

Sand, gravel, and rock

Shale

Sand and gravel

Clay

Sand and gravel

Sand and rock

Shale

Sand and rock

Clay

Sand

Clay

Hell JY-65-26-614

Owner: Allied Concrete

Driller: G. S. Rhemann Hater Hell Service, Inc.

\begin{tabular}{|c|c|c|}
\hline Description & $\begin{array}{l}\text { Thick- } \\
\text { ness } \\
\text { (feet) }\end{array}$ & $\begin{array}{c}\text { Depth } \\
\text { interval } \\
\text { (feet) }\end{array}$ \\
\hline Topsoil & 4 & $0-4$ \\
\hline Clay & 8 & 4- 12 \\
\hline Loam, sandy & 8 & $12-20$ \\
\hline Sand & 34 & $20-54$ \\
\hline Clay, gray & 33 & $54-87$ \\
\hline Sand and gravel & 9 & $87-96$ \\
\hline Clay, blue & 4 & $96-100$ \\
\hline Clay, red & 38 & $100-138$ \\
\hline Sand & 56 & $138-194$ \\
\hline Clay, tan & 18 & $194-212$ \\
\hline Clay and rocky clay & 80 & $212-292$ \\
\hline Sand, silty & 80 & $292-372$ \\
\hline Clay, hard & 16 & $372-388$ \\
\hline Sand, coarse & 50 & $388-438$ \\
\hline Clay, rocky & 8 & $438-446$ \\
\hline Sand, fine & 14 & $446-460$ \\
\hline Clay, white & 2 & $460-462$ \\
\hline Clay, rocky & 12 & $462-474$ \\
\hline
\end{tabular}

Hell JY-65-26-614--Continued

Sand, coarse

$2 \quad 474-476$

Clay, pink

21

476-497

Sand, gold

21

497-518

Stone layers

$27 \quad 518-545$

Sand, coarse

545-554

Hell JY-65-26-813

Owner: Lamar Consol idated Independent School District, Meyer Intermediate School

Driller: Birdwell Water Well Service Co., Inc.

\begin{tabular}{|c|c|c|}
\hline Description & $\begin{array}{c}\text { Thick- } \\
\text { ness } \\
\text { (feet) }\end{array}$ & $\begin{array}{c}\text { Depth } \\
\text { interval } \\
\text { (feet) }\end{array}$ \\
\hline Clay & 20 & $0-20$ \\
\hline Sand, brown & 17 & $20-37$ \\
\hline Clay & 1 & $37-38$ \\
\hline Sand & 10 & $38-48$ \\
\hline Clay & 2 & 48- 50 \\
\hline Gravel & 27 & $50-77$ \\
\hline Clay & 23 & $77-100$ \\
\hline Rock & 3 & $100-103$ \\
\hline Clay, sandy & 5 & $103-108$ \\
\hline Sand & 6 & $108-114$ \\
\hline Clay & 26 & $114-140$ \\
\hline Sand, coarse, brown & 10 & $140-150$ \\
\hline Clay & 40 & $150-190$ \\
\hline Sand & 30 & $190-220$ \\
\hline Gravel & 10 & $220-230$ \\
\hline Clay & 26 & $230-256$ \\
\hline Sand & 34 & $256-290$ \\
\hline Gravel & 10 & $290-300$ \\
\hline Sand & 24 & $300-324$ \\
\hline Sand, broken & 2 & $324-326$ \\
\hline Sand & 9 & $326-335$ \\
\hline Clay & 13 & $335-348$ \\
\hline Sand & 4 & $348-352$ \\
\hline Slay & 20 & $352-372$ \\
\hline Sand, good, coarse & 56 & $372-428$ \\
\hline Clay & 3 & $428-431$ \\
\hline Sand and gravel & 14 & $431-445$ \\
\hline Clay & 15 & $445-460$ \\
\hline
\end{tabular}


Well JY-65-26-813--Cont inued

\begin{tabular}{|c|c|c|}
\hline Sand, good & 12 & $460-472$ \\
\hline Clay & 13 & $472-485$ \\
\hline Sand & 18 & $485-503$ \\
\hline Clay & 21 & $503-524$ \\
\hline Sand & 33 & $524-557$ \\
\hline Clay & 3 & $557-560$ \\
\hline Sand & 23 & $560-583$ \\
\hline Clay & 5 & $583-588$ \\
\hline Sand & 10 & $588-598$ \\
\hline Clay & 16 & $598-614$ \\
\hline Sand, good & 31 & $614-645$ \\
\hline Sand, fair & 9 & $645-654$ \\
\hline Sand, good & 15 & $654-669$ \\
\hline Clay & 7 & $669-676$ \\
\hline Sand & 32 & $676-708$ \\
\hline Clay & 19 & $708-727$ \\
\hline Sand & 3 & $727-730$ \\
\hline Clay & 4 & $730-734$ \\
\hline Sand, poor & 10 & $734-744$ \\
\hline Sand, fair & 12 & $744-756$ \\
\hline Sand, fair, broken streaks & 10 & $756-766$ \\
\hline Sand, good & 10 & $766-776$ \\
\hline Sand & 1 & $776-777$ \\
\hline Clay & 51 & $777-828$ \\
\hline Clay, sandy & 3 & $828-831$ \\
\hline Clay & 7 & $831-838$ \\
\hline Sand, good & 14 & $838-852$ \\
\hline Sand, fine, poor & 3 & $852-855$ \\
\hline Sand, good & 7 & $855-862$ \\
\hline Clay & 17 & $862-879$ \\
\hline Clay, sandy & 9 & $879-888$ \\
\hline Sand & 44 & $888-932$ \\
\hline Clay & 5 & $932-937$ \\
\hline
\end{tabular}

Hell JY-65-27-106

Owner: Pecan Grove Municipal Utility District, Hell No. 1

Driller: Layne-Texas Co.

\begin{tabular}{|c|c|c|c|}
\hline Description & $\begin{array}{l}\text { Thick- } \\
\text { ness } \\
\text { (feet) }\end{array}$ & $\begin{array}{r}\text { Dep } \\
\text { inte } \\
\text { (fe }\end{array}$ & $\begin{array}{l}\text { th } \\
\text { rval } \\
\text { et) }\end{array}$ \\
\hline Clay & 20 & $0-$ & 20 \\
\hline Sand and gravel & 6 & $20-$ & 26 \\
\hline Sand & 15 & 26- & 41 \\
\hline Boulder & 2 & 41- & 43 \\
\hline Sand and boulder & 6 & 43- & 49 \\
\hline Clay, red & 13 & 49- & 62 \\
\hline Sand and clay streaks & 29 & $62-$ & 91 \\
\hline clay, red & 14 & $91-$ & 105 \\
\hline Sand & 25 & $105-$ & 130 \\
\hline Clay & 28 & $130-$ & 158 \\
\hline Sand & 9 & $158-$ & 167 \\
\hline Clay & 21 & $167-$ & 188 \\
\hline Sand and clay breaks & 62 & $188-$ & 250 \\
\hline Shale, sandy & 47 & $250-$ & 297 \\
\hline Sand and gravel & 6 & $297-$ & 303 \\
\hline Sand, gravel and shale breaks & 44 & 303- & 347 \\
\hline Shale and sand streaks & 55 & $347-$ & 402 \\
\hline Sand & 6 & 402- & 408 \\
\hline Sand and streaks of gray shale & 97 & 408- & 505 \\
\hline Gravel, hard & 12 & $505-$ & 517 \\
\hline Shale, sticky & 44 & $517-$ & 561 \\
\hline Sand, gravel and streaks of clay & 58 & $561-$ & 619 \\
\hline Sand and clay breaks & 21 & $619-$ & 640 \\
\hline Shale, hard with sand breaks & 30 & $640-$ & 670 \\
\hline Shale and sandy breaks & 35 & $670-$ & 705 \\
\hline Sand and shale breaks & 120 & $705-$ & 825 \\
\hline Shale & 15 & $825-$ & 840 \\
\hline Sand & 34 & $840-$ & 874 \\
\hline Shale, brown & 146 & $874-1$ & .020 \\
\hline Sand & 13 & $1,020-1$ & .033 \\
\hline Shale & 23 & $1,033-1$ &, 056 \\
\hline Shale, blue and sand layers & 42 & $1,056-1$ & .098 \\
\hline Shale, hard brown & 20 & $1,098-1$ &, 118 \\
\hline Sand and shale breaks & 45 & $1,118-1$ &, 163 \\
\hline
\end{tabular}


Well JY-65-27-106--Continued

Shale and sand layers

$57 \quad 1,163-1,220$

Sand

Shale

Shale and sand shale

Shale and broken sand

Shale

Sand and shale layers

Shale, hard brown

Sand

Shale, brown

Sand

Shale with sandy streaks

Well JY-65-27-107

Owner: Pecan Grove Associates, Well No. 1

Driller: G. S. Rhemann Water Well Service, Inc.

\begin{tabular}{|c|c|c|c|}
\hline Description & $\begin{array}{l}\text { Thick- } \\
\text { ness } \\
\text { (feet) }\end{array}$ & $\begin{array}{l}\text { Def } \\
\text { inte } \\
\text { (ff }\end{array}$ & $\begin{array}{l}\text { th } \\
\text { rval } \\
\text { et) }\end{array}$ \\
\hline Gumbo, brown & 14 & $0-$ & 14 \\
\hline Clay, $\tan$ sandy & 26 & 14- & 40 \\
\hline Sand, silty and gravel & 15 & 40- & 55 \\
\hline Clay, brown & 25 & $55-$ & 80 \\
\hline Sand and gravel & 8 & $80-$ & 88 \\
\hline Clay, reddish white & 12 & 88- & 100 \\
\hline Sand and gravel & 42 & $100-$ & 142 \\
\hline Clay, brown & 3 & $142-$ & 145 \\
\hline Sand, dirty and gravel & 28 & 145- & 173 \\
\hline Clay, white & 7 & $173-$ & 180 \\
\hline Sand, very fine & 20 & $180-$ & 200 \\
\hline Clay, white & 27 & 200- & 227 \\
\hline Clay and stone layers & 24 & 227- & 251 \\
\hline Sand, coarse and gravel & 27 & 251- & 278 \\
\hline Clay & 4 & 278- & 282 \\
\hline Sand, coarse & 14 & $282-$ & 296 \\
\hline Clay & 4 & 296- & 300 \\
\hline Sand, coarse & 13 & $300-$ & 313 \\
\hline
\end{tabular}

Well JY-65-27-108

Owner: Pecan Grove Municipal Utility District, Well No. 2

Driller: Layne-Texas Co.

\begin{tabular}{|c|c|c|}
\hline Description & $\begin{array}{c}\text { Thick- } \\
\text { ness } \\
\text { (feet) }\end{array}$ & $\begin{array}{c}\text { Depth } \\
\text { interval } \\
\text { (feet) }\end{array}$ \\
\hline Clay & 30 & $0-30$ \\
\hline Sand & 15 & $30-45$ \\
\hline Clay & 64 & 45-109 \\
\hline Sand & 32 & $109-141$ \\
\hline Clay & 20 & $141-161$ \\
\hline Clay, sandy & 6 & $161-167$ \\
\hline Sand & 6 & $167-173$ \\
\hline Clay & 17 & $173-190$ \\
\hline Sand & 12 & $190-202$ \\
\hline Clay & 27 & $202-229$ \\
\hline Sand & 58 & $229-287$ \\
\hline Clay & 7 & $287-294$ \\
\hline Sand & 32 & $294-326$ \\
\hline Clay & 22 & $326-348$ \\
\hline Sand & 14 & $348-362$ \\
\hline Clay, sandy & 8 & $362-370$ \\
\hline Clay & 34 & $370-404$ \\
\hline Sand & 3 & $404-407$ \\
\hline Clay & 16 & $407-423$ \\
\hline Sand & 20 & $423-443$ \\
\hline Clay & 7 & $443-450$ \\
\hline Sand and fine gravel & 64 & $450-514$ \\
\hline Clay streaks, sandy & 3 & $514-517$ \\
\hline Sand and fine gravel & 20 & $517-537$ \\
\hline Clay, sandy & 4 & $537-541$ \\
\hline Sand & 11 & $541-552$ \\
\hline \multicolumn{3}{|c|}{$\begin{array}{l}\text { Well JY-65-27-109 } \\
\text { Owner: Lois Clark } \\
\text { Driller: Mahler Water Well Service, In }\end{array}$} \\
\hline Description & $\begin{array}{l}\text { Thick- } \\
\text { ness } \\
\text { (feet) }\end{array}$ & $\begin{array}{c}\text { Depth } \\
\text { interval } \\
\text { (feet) }\end{array}$ \\
\hline Topsoil & 5 & $0-5$ \\
\hline silt & 5 & $5-10$ \\
\hline Clay, red & 30 & $10-40$ \\
\hline Sand & 15 & $40-55$ \\
\hline
\end{tabular}


Hell JY-65-27-109--Cont inued

$\begin{array}{lll}\text { Shale, red } & 10 & 55-65 \\ \text { Sand } & 25 & 65-90 \\ \text { Shale, white } & 10 & 90-100 \\ \text { Shale, red } & 10 & 100-110 \\ \text { Sand and shale } & 20 & 110-130 \\ \text { Sand } & 30 & 130-160 \\ \text { Sand and shale } & 10 & 160-170 \\ \text { Sand } & 15 & 170-185 \\ \text { Shale, red } & 20 & 185-205 \\ \text { Sand } & 20 & 205-225 \\ \text { Shale, white and sand } & 15 & 225-240 \\ \text { Sand } & 28 & 240-268\end{array}$

Hell JY-65-27-212

Owner: Robert Schumann

Driller: Ellis Water Well Drilling

\begin{tabular}{lcr}
\hline \multicolumn{1}{c}{ Description } & $\begin{array}{c}\text { Thick- } \\
\text { ness } \\
\text { (feet) }\end{array}$ & $\begin{array}{c}\text { Depth } \\
\text { interval } \\
\text { (feet) }\end{array}$ \\
\hline Topsoil, black & 3 & $0-3$ \\
Soil, red-to-gray clay & 6 & $3-9$ \\
Sand, brown bank & 24 & $9-33$ \\
Clay & 3 & $33-36$ \\
Sand, coarse-to-gravel & 41 & $46-77$ \\
Sand, streaks and red clay & 28 & $77-105$ \\
Sand, fine-to-coarse & 15 & $105-120$ \\
Clay, red and sand streaks & 50 & $120-170$ \\
Sand, coarse-to-gravel & 20 & $170-190$ \\
Clay, red wi th sand layers & 23 & $190-213$ \\
Sand, coarse and gravel & 20 & $213-233$ \\
Clay, red & 7 & $233-240$ \\
Sand, coarse and gravel & 10 & $240-250$ \\
Clay, red, sticky-to-gray & 52 & $250-302$ \\
Sand, coarse and gravel & 27 & $302-329$
\end{tabular}

Hell JY-65-27-213

Owner: Fort Bend County Municipal Utility District 69, Hell No. 1

Driller: Alsay Incorporated

\begin{tabular}{|c|c|c|c|}
\hline Description & $\begin{array}{l}\text { Thick- } \\
\text { ness } \\
\text { (feet) }\end{array}$ & $\begin{array}{l}\text { Dept| } \\
\text { inter } \\
\text { (fee }\end{array}$ & $\begin{array}{l}\text { th } \\
\text { rval } \\
\text { et) }\end{array}$ \\
\hline Topsoil & 4 & $0-$ & 4 \\
\hline Clay, brown & 18 & 4- & 22 \\
\hline Sand & 14 & $22-$ & 36 \\
\hline Gravel, coarse & 43 & $36-$ & 79 \\
\hline Clay, brown & 35 & $79-$ & 114 \\
\hline Sand & 15 & $114-$ & 129 \\
\hline Clay, brownish gray & 45 & $129-$ & 174 \\
\hline Sand & 85 & $174-$ & 259 \\
\hline Clay, gray & 15 & 259- & 274 \\
\hline Sand & 44 & 274- & 318 \\
\hline Clay, gray & 6 & $318-$ & 324 \\
\hline Gravel, fine & 12 & $324-3$ & 336 \\
\hline Clay, blue & 62 & $336-$ & 398 \\
\hline Sand & 50 & $398-$ & 448 \\
\hline Clay, tan & 26 & $448-$ & 474 \\
\hline Sand & 10 & 474- & 484 \\
\hline Clay, $\tan$ & 12 & 484- & 496 \\
\hline Sand with tan clay streaks & 72 & 496-: & 568 \\
\hline Clay, tan & 10 & $568-$ & 578 \\
\hline Sand & 90 & 578- & 668 \\
\hline Clay, $\tan$ & 30 & $668-$ & 698 \\
\hline Sand & 36 & $698-7$ & 734 \\
\hline Clay, whitish tan & 8 & $734-7$ & 742 \\
\hline $\begin{array}{l}\text { Sand with small streaks of whitish } \\
\text { tan clay }\end{array}$ & 212 & $742-$ & 954 \\
\hline Clay, gray & 30 & $954-\varsigma$ & 984 \\
\hline Sand & 22 & $984-1$, & 006 \\
\hline Clay, whitish tan & 10 & $1,006-1,0$ & 016 \\
\hline Sand & 16 & $1,016-1,0$ & 032 \\
\hline Clay, gray & 44 & $1,032-1$, & 076 \\
\hline Sand & 32 & $1,076-1$ & 108 \\
\hline Clay, gray & 14 & $1,108-1$, & 122 \\
\hline Sand & 10 & $1,122-1$, & 132 \\
\hline Clay, gray & 54 & $1,132-1$, & 186 \\
\hline Sand, with whitish tan clay & 52 & $1,186-1,2$ & 238 \\
\hline
\end{tabular}


Well JY-65-27-213--Cont inued

$\begin{array}{lcc}\text { Clay } & 14 & 1,238-1,252 \\ \text { Sand } & 34 & 1,252-1,286 \\ \text { Clay, gray } & 32 & 1,286-1,318 \\ \text { Sand with clay breaks } & 26 & 1,318-1,344 \\ \text { Clay, gray } & 70 & 1,344-1,414 \\ \text { Sand, hard } & 18 & 1,414-1,432 \\ \text { Clay, gray } & 62 & 1,432-1,494\end{array}$

Well JY-65-27-319

Owner: Venetian Estates

Driller: Layne-Texas Co.

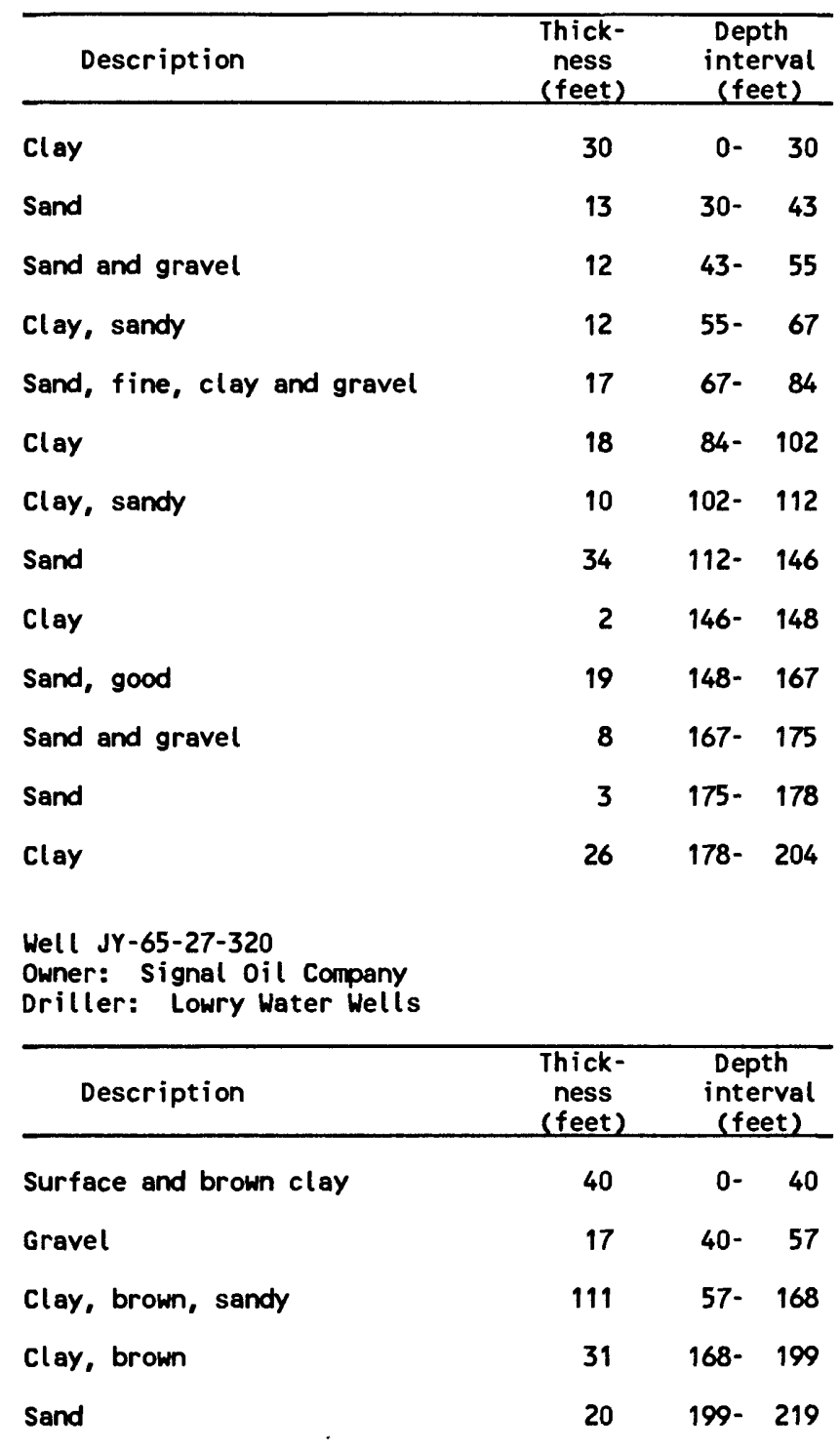

Well JY-65-27-321

Owner: Brazos River Authority

Driller: Layne-Western Company, Inc.

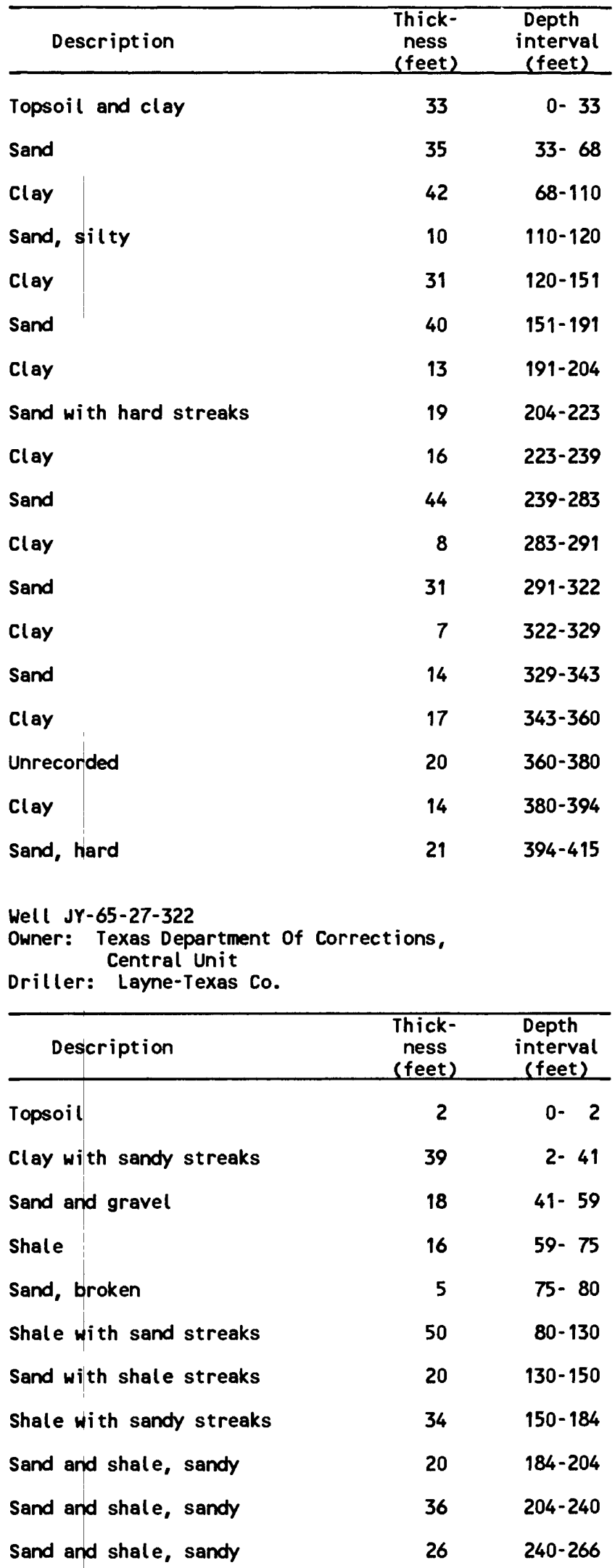


Table 3.--Drillers' logs of selected wells in Fort Bend County, 1969-87--Cont inued

Well JY-65-27-322--Cont inued

Shale

Sand and shale

16

266-282

Sand and small gravel

25

282-307

Shale

28

307-335

10

335-345

Sand and small gravel

49

345-394

Shale

11

$394-405$

Well JY-65-27-323

Owner: Fort Bend County Municipal Utility District 13, Well No. 3

Driller: Layne-Texas Co.

\begin{tabular}{|c|c|c|}
\hline Description & $\begin{array}{l}\text { Thick- } \\
\text { ness } \\
\text { (feet) }\end{array}$ & $\begin{array}{c}\text { Depth } \\
\text { interval } \\
\text { (feet) }\end{array}$ \\
\hline Topsoil & 3 & $0-3$ \\
\hline Clay, fill & 4 & 3- 7 \\
\hline Clay & 3 & $7-10$ \\
\hline Sand and clay streaks & 3 & $10-13$ \\
\hline Sand & 2 & $13-15$ \\
\hline Sand and $\mathrm{clay}$ streaks & 5 & $15-20$ \\
\hline Clay & 21 & $20-41$ \\
\hline Ctay and sand streaks & 40 & $41-81$ \\
\hline Sand and gravel & 19 & $81-100$ \\
\hline Clay & 15 & $100-115$ \\
\hline Sand and clay & 36 & $115-151$ \\
\hline Clay and sandy streaks & 4 & $151-155$ \\
\hline Sand & 7 & $155-162$ \\
\hline Clay and sand streaks & 7 & $162-169$ \\
\hline Sand & 33 & $169-202$ \\
\hline Shale and sand streaks & 17 & $202-219$ \\
\hline Sand & 35 & $219-254$ \\
\hline Shale and sand streaks & 23 & $254-277$ \\
\hline Sand and shale streaks & 10 & $277-287$ \\
\hline Shale & 23 & $287-310$ \\
\hline Gravel, shale streaks and sand & 6 & $310-316$ \\
\hline Shale & 26 & $316-342$ \\
\hline Sand, shale streaks & 11 & $342-353$ \\
\hline Clay & 9 & $353-362$ \\
\hline $\mathrm{Clay}$ and sand streaks & 45 & $362-407$ \\
\hline Sand, shale and gravel & 13 & $407-420$ \\
\hline
\end{tabular}

Well JY-65-27-323--Cont inued

Shale $\quad 15$

$420-435$

Gravel and sand

435- $\quad 467$

Sand and gravel

467- $\quad 478$

Shale

478- 531

Sand and shale

531- 547

Shale

547- 648

Sand and shale streaks

648- 661

Shale and sand streaks

$661-676$

Sand and shole streaks

300

676- 976

Shale

Sand and shale streaks

3 976- 979

Shale and sand streaks

$77 \quad 979-1,056$

$141,056-1,070$

Shale

$70 \quad 1,070-1,140$

Shale and sand streaks

$50 \quad 1,140-1,190$

Sand and shale streaks

$19 \quad 1,190-1,209$

Shale

$17 \quad 1,209-1,226$

Shale, sandy

$131,226-1,239$

Shale

Sand

Shale, sandy

$34 \quad 1,239-1,273$

7 1,273-1,280

$51,280-1,285$

Sand and shale streaks

$33 \quad 1,285-1,318$

Shale

$40 \quad 1,318-1,358$

Sand and shale streaks

$23 \quad 1,358-1,381$

Shale

$21 \quad 1,381-1,402$

Sand and shale streaks

$241,402-1,426$

Shale, hard

$14 \quad 1,426-1,440$

Shale

$31 \quad 1,440-1,471$

Sand and shale streaks

$29 \quad 1,471-1,500$

Shale

$61,500-1,506$

Well JY-65-27-324

Owner: Fort Bend Utilities, Well No. 10 Driller: Layne-Texas Co.

\begin{tabular}{lccr}
\hline Description & $\begin{array}{c}\text { Thick- } \\
\text { ness } \\
\text { (feet) }\end{array}$ & $\begin{array}{c}\text { Depth } \\
\text { interval } \\
\text { (feet) }\end{array}$ \\
\hline Topsoil & 2 & $0-$ & 2 \\
Clay & 5 & $2-$ & 7 \\
Sand and gravel & 75 & $7-$ & 82 \\
Clay & 17 & $82-$ & 99
\end{tabular}


Well JY-65-27-324--Cont inued

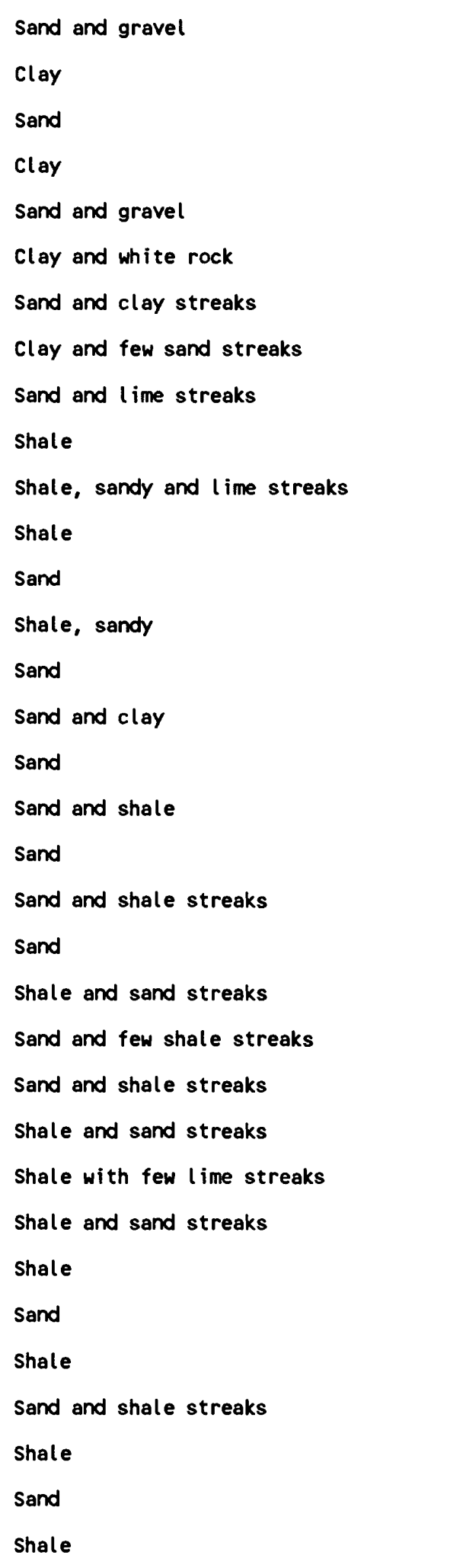

Well JY-65-27-405

Owner: J. C. Wessendorf

$23 \quad 99-122$

7 122- 129

$53 \quad 129-182$

$14 \quad 182-196$

$16 \quad 196-212$

$48 \quad 212-260$

$78 \quad 260-338$

$24 \quad 338-362$

$32 \quad 362-394$

$29 \quad 394-423$

28 423- 451

11 451- 462

47 462- 509

15

509- 524

$27 \quad 524-551$

$17 \quad 551-568$

34

568- 602

15

602- 617

14

617- 631

16

631- 647

15

647- 662

23

662- 685

$88 \quad 685-773$

$43 \quad 773-816$

49 816- 865

$36 \quad 865-901$

51 901- 952

$22 \quad 952-974$

12

974- 986

$14986-1,000$

$20 \quad 1,000-1,020$

$71 \quad 1,020-1,091$

$11 \quad 1,091-1,102$

$47 \quad 1,102-1,149$

Well JY-65-27-505
Driller: Hardin Water Wells

\begin{tabular}{|c|c|c|}
\hline Description & $\begin{array}{c}\text { Thick- } \\
\text { ness } \\
\text { (feet) }\end{array}$ & $\begin{array}{c}\text { Depth } \\
\text { interval } \\
\text { (feet) }\end{array}$ \\
\hline Sand, surface & 3 & $0-3$ \\
\hline clay, red & 32 & $3-35$ \\
\hline Sand and gravel & 25 & $35-60$ \\
\hline Clay, red and white & 5 & $60-65$ \\
\hline Sand and gravel & 20 & $65-85$ \\
\hline \multicolumn{3}{|c|}{$\begin{array}{l}\text { Well JY- } 65-27-504 \\
\text { Owner: Plantation Municipal Utility } \\
\text { District, Well No. } 1 \\
\text { Driller: Water Resources of Texas }\end{array}$} \\
\hline Description & $\begin{array}{l}\text { Thick- } \\
\text { ness } \\
\text { (feet) }\end{array}$ & $\begin{array}{c}\text { Depth } \\
\text { interval } \\
\text { (feet) }\end{array}$ \\
\hline Unrecorded & 561 & $0-561$ \\
\hline Shale & 8 & $561-569$ \\
\hline Sand, good & 160 & $569-729$ \\
\hline Shale & 30 & $729-759$ \\
\hline Sand, good & 40 & $759-799$ \\
\hline Shale & 10 & $799-809$ \\
\hline
\end{tabular}

Owner: Plantation Municipal Utility District, Well No. 2

Driller: Landford Drilling Co., Inc.

\begin{tabular}{lcc}
\hline \multicolumn{1}{c}{ Description } & $\begin{array}{c}\text { Thick- } \\
\text { ness } \\
\text { (feet) }\end{array}$ & $\begin{array}{c}\text { Depth } \\
\text { interval } \\
\text { (feet) }\end{array}$ \\
\hline Sand & 3 & $0-3$ \\
Clay & 11 & $3-14$ \\
Sand & 26 & $14-40$ \\
Shale, sandy & 110 & $40-150$ \\
Sand & 50 & $150-200$ \\
Shale & 40 & $200-240$ \\
Sand & 30 & $240-270$ \\
Shale & 20 & $270-290$ \\
Sand & 140 & $290-430$ \\
Shale & 10 & $430-440$ \\
Sand & 45 & $440-485$ \\
Shale & 50 & $485-535$ \\
Sand & 25 & $535-560$
\end{tabular}


Well JY-65-27-505--Cont inued

Shale
Sand
Shale
Sand
Shale
Shale, sandy and shale
Well JY-65-27-609
Owner: Greystone Construction Company
Driller: Bussell and Son, Inc.

\begin{tabular}{lcc}
\hline \multicolumn{1}{c}{ Description } & $\begin{array}{c}\text { Thick- } \\
\text { ness } \\
\text { (feet) }\end{array}$ & $\begin{array}{c}\text { Depth } \\
\text { interval } \\
\text { (feet) }\end{array}$ \\
\hline Clay & 3 & $0-3$ \\
Sand & 28 & $3-31$ \\
Clay & 5 & $31-36$ \\
Gravel & 94 & $36-130$ \\
Clay & 20 & $130-150$ \\
Sand & 37 & $150-187$ \\
Clay & 80 & $187-267$ \\
Sand & 18 & $267-285$ \\
Clay & 17 & $285-302$ \\
Clay, sandy & 18 & $302-320$ \\
Sand & 26 & $320-346$ \\
Clay & 22 & $346-368$ \\
Sand & 16 & $368-384$ \\
Clay & 72 & $384-391$ \\
Sand & $391-463$
\end{tabular}

Well JY-65-28-102

Owner: City of Cities Municipal Utility

District, Sugar Creek, Well No. 1

Driller: Layne-Texas Co.

\begin{tabular}{rr}
22 & $560-582$ \\
158 & $582-740$ \\
50 & $740-790$ \\
40 & $790-830$ \\
10 & $830-840$ \\
70 & $840-910$ \\
\hline
\end{tabular}

\begin{tabular}{lcc}
\hline \multicolumn{1}{c}{ Description } & $\begin{array}{c}\text { Thick- } \\
\text { ness } \\
\text { (feet) }\end{array}$ & $\begin{array}{c}\text { Depth } \\
\text { interval } \\
\text { (feet) }\end{array}$ \\
\hline Topsoil & 2 & $0-2$ \\
Sand & 18 & $2-20$ \\
Clay, streaks of sand & 69 & $20-89$ \\
Sand & 35 & $89-124$ \\
Shale & 23 & $124-147$ \\
Sand & 13 & $147-160$ \\
Shale and sand streaks & 33 & $160-193$
\end{tabular}

Hell JY-65-28-102--Cont inued

Sand $\quad 58$

193- 251

Sand and shale streaks

30

251- 281

Sand, hard and clay

281- 311

Shale

Sand and shale streaks

$311-322$

Sand

Shale and sand streaks

322- 342

Sand and shale streaks

342- 354

Shale

354- 402

50

402- 452

20

452- 472

Sand

23

472- 495

Sand, coarse

81

495- 576

Shale

Shale and sand

10

576- 586

Sand, coarse

586- 617

Shale, hard, coarse sand

617- 649

Sand, hard, coarse

$649-680$

Shale

$680-690$

Sand

Shale

Shale, sandy

Sand and shale streaks

$690-716$

44

716- 760

16

$760-776$

26

776- 802

Shale

802- 854

Shale, sandy

854- 864

Sand

Sand and shale

864- 894

Shale

894- 946

Shale, sandy

946- 973

Shale

973- 985

$61985-1,046$

$201,046-1,066$

Well JY-65-28-105

Owner: Fort Bend County Municipal Utility District 13, Well No. 2

Driller: Layne-Texas Co.

\begin{tabular}{lccc}
\hline Description & $\begin{array}{c}\text { Thick- } \\
\text { ness } \\
\text { (feet) }\end{array}$ & $\begin{array}{c}\text { Depth } \\
\text { interval } \\
\text { (feet) }\end{array}$ \\
\hline Topsoil & 3 & $0-$ & 3 \\
Clay & 6 & $3-$ & 9 \\
Clay and sand streaks & 25 & $9-$ & 34 \\
Clay & 4 & $34-$ & 38
\end{tabular}


Well JY-65-28-105--Cont inued

Sand and gravel

Clay

Sand and gravel

Sand and clay streaks

Shale and sand streaks

Sand and shale streaks

Shale and sand streaks

Sand

Shale

Sand and shale streaks

Shale and sand streaks

Shale

Sand and shale streaks

Shale

Sand and shale streaks

Shale

Shale and sand streaks

Sand

Shale

Sand, gravel and shale streaks

Shale

Sand, gravel and shale streaks

Shale

Sand, shale and gravel

Sand and shale streaks

Shale and sand streaks

Clay, sandy

Clay

Sand and clay streaks

Sand and shale streaks

Shale

Sand and shale streaks

Shale

Shale and sand streaks

Sand and shale streaks

Shale

Sand and shale streaks
Well JY-65-28-105--Cont inued

Shale

$13 \quad 1,312-1,325$

Sand and shale streaks

$24 \quad 1,325-1,349$

Shale

$27 \quad 1,349-1,376$

Sand and shale streaks

$191,376-1,395$

Shale and sand streaks

$351,395-1,430$

Sand and shale

$631,430-1,493$

Shale

$7 \quad 1,493-1,500$

Well JY-65-28-106

Owner: Land and Water Ammenities

Driller: Robinson Water Well Service, Inc.

\begin{tabular}{lcrr}
\hline Description & $\begin{array}{c}\text { Thick- } \\
\text { ness } \\
\text { (feet) }\end{array}$ & $\begin{array}{c}\text { Depth } \\
\text { interval } \\
\text { (feet) }\end{array}$ \\
\hline Clay & 13 & $0-13$ \\
Sand and gravel & 87 & $13-100$ \\
Clay & 89 & $100-189$ \\
Sand & 41 & $189-230$ \\
Clay & 41 & $230-271$ \\
Sand & 75 & $271-346$ \\
Clay & 54 & $346-400$ \\
Sand & 10 & $400-410$ \\
Clay & 20 & $410-$ & 430 \\
Sand & 71 & $430-$ & 501
\end{tabular}

Well JY-65-28-107

Owner: Greystone Construction Company

Driller: Robinson Water Hell Service, Inc.

\begin{tabular}{l|rrr}
\hline \multicolumn{2}{r}{ Description } & $\begin{array}{c}\text { Thick- } \\
\text { ness } \\
\text { (feet) }\end{array}$ & $\begin{array}{c}\text { Depth } \\
\text { interval } \\
\text { (feet) }\end{array}$ \\
\hline Clay & 12 & $0-$ & 12 \\
Sand & 72 & $12-84$ \\
Clay & 94 & $84-$ & 178 \\
Sand & 69 & $178-247$ \\
Clay & 133 & $247-380$ \\
Sand & 80 & $380-460$ \\
Clay & 20 & $460-480$ \\
Sand & 56 & $480-536$
\end{tabular}


Well JY-65-28-108

Owner: Kaneb Services

Driller: Bussell and Son, Inc.

\begin{tabular}{lcc}
\hline \multicolumn{1}{c}{ Description } & $\begin{array}{c}\text { Thick- } \\
\text { ness } \\
\text { (feet) }\end{array}$ & $\begin{array}{c}\text { Depth } \\
\text { interval } \\
\text { (feet) }\end{array}$ \\
\hline Clay, red & 2 & $0-2$ \\
Sand, surface & 37 & $2-39$ \\
Clay, soft & 99 & $39-138$ \\
Sand & 37 & $138-175$ \\
Clay & 50 & $175-225$ \\
Sand & 31 & $225-256$ \\
Clay & 44 & $256-300$ \\
Sand, fine & 20 & $300-320$ \\
Clay & 36 & $320-356$ \\
Sand & 8 & $356-364$ \\
Clay, sandy & 69 & $364-433$ \\
Sand & 14 & $433-447$ \\
Clay & 18 & $447-465$ \\
Sand & 28 & $465-493$ \\
Clay & 7 & $493-500$ \\
Sand & 50 & $500-550$
\end{tabular}

Hell JY-65-28-207

Owner: Meadow Creek Municipal Utility District

Driller: Layne-Texas Co.

\begin{tabular}{|c|c|c|c|c|c|c|}
\hline $\begin{array}{l}\text { Owner: Meadow Creek Munici } \\
\text { District } \\
\text { Driller: Layne-Texas Co. }\end{array}$ & lity & & Description & $\begin{array}{l}\text { Thick- } \\
\text { ness } \\
\text { (feet) }\end{array}$ & $\begin{array}{l}\text { De } \\
\text { int } \\
\text { (f }\end{array}$ & $\begin{array}{l}\text { th } \\
\text { rval } \\
\text { et) }\end{array}$ \\
\hline Description & $\begin{array}{l}\text { Thick- } \\
\text { ness } \\
\text { (feet) }\end{array}$ & $\begin{array}{l}\text { Depth } \\
\text { interval } \\
\text { (feet) }\end{array}$ & $\begin{array}{l}\text { Topsoil } \\
\text { Clay, sandy }\end{array}$ & $\begin{array}{r}3 \\
38\end{array}$ & $\begin{array}{l}0- \\
3-\end{array}$ & $\begin{array}{r}3 \\
41\end{array}$ \\
\hline Topsoil & 5 & $0-5$ & Sand & 65 & $41-$ & 106 \\
\hline Clay & 20 & $5-25$ & Clay & 13 & $106-$ & 119 \\
\hline Sand & 30 & $25-55$ & Clay, sandy and sand & 15 & $119-$ & 134 \\
\hline Clay & 40 & $55-95$ & Clay & 32 & 134- & 166 \\
\hline Sand & 25 & $95-120$ & Clay, sandy & 10 & $166-$ & 176 \\
\hline Shale & 89 & $120-209$ & Sand and sandy clay & 48 & 176- & 224 \\
\hline Sand and shale streaks & 21 & $209-230$ & Clay & 62 & $224-$ & 286 \\
\hline Shale and few sand streaks & 53 & $230-283$ & Shale, sandy & 4 & 286- & 290 \\
\hline Shale & 64 & $283-347$ & Shale & 10 & $290-$ & 300 \\
\hline Shale and sand streaks & 21 & $347-368$ & Shale, sandy & 40 & $300-$ & 340 \\
\hline Shale & 107 & $368-475$ & Sand and sandy shale streaks & 27 & $340-$ & 367 \\
\hline Shale and sand streaks & 117 & $475-592$ & Shale, sandy & 14 & $367-$ & 381 \\
\hline Sand & 65 & $592-657$ & Sand & 11 & $381-$ & 392 \\
\hline
\end{tabular}

Well JY-65-28-207--Continued

Shale and sand streaks $\quad 15$

$657-672$

Sand

58

672- 730

Shale

$5730-735$

Sand and shale streaks

$20 \quad 735-755$

Shale and sand streaks

20

$755-775$

Sand

$45 \quad 775-820$

Sand and shale streaks

50

$820-870$

Sand

36

Sand and shale streaks with lime

48

870- 906

Sand

$31954-985$

Sand, shale and lime streaks

$47 \quad 985-1,032$

Sand

$17 \quad 1,032-1,049$

Shale

Sand and shale streaks

$5 \quad 1,049-1,054$

$261,054-1,080$

Shale

Sand with shale and lime streaks

$41,080-1,084$

Shale

$33 \quad 1,084-1,117$

$18 \quad 1,117-1,135$

Hell JY-65-28-208

Owner: Quail Valley Utility District.

Driller: Layne-Texas Co. Hell No. 3 
Table 3.--Drillers' logs of selected wells in Fort Bend County, 1969-87--Cont inued

Well JY-65-28-208--Continued

\begin{tabular}{|c|c|c|}
\hline Shale & 3 & $392-395$ \\
\hline Sand and shale streaks & 7 & $395-\quad 402$ \\
\hline Shale & 20 & $402-\quad 422$ \\
\hline Sand and shale & 30 & $422-\quad 452$ \\
\hline Shale & 12 & $452-\quad 464$ \\
\hline Sand and sandy shale & 26 & $464-\quad 490$ \\
\hline Shale, sandy and sand streaks & 47 & $490-\quad 537$ \\
\hline Sand and sandy shale & 33 & $537-570$ \\
\hline Shale & 53 & $570-623$ \\
\hline Shale, sandy & 5 & $623-628$ \\
\hline Shale & 19 & $628-647$ \\
\hline Shale, sandy & 32 & $647-679$ \\
\hline Shale & 13 & $679-692$ \\
\hline Shale, sandy and sand streaks & 62 & $692-754$ \\
\hline Sand and sandy shale & 56 & $754-810$ \\
\hline Shale and sand layers & 42 & $810-852$ \\
\hline Sand, l ime and shale & 32 & $852-884$ \\
\hline Sand, gravel and shale & 41 & $884-925$ \\
\hline Shale, sandy and sand streaks & 76 & $925-1,001$ \\
\hline Sand and shale streaks & 11 & $1,001-1,012$ \\
\hline Shale and sandy streaks & 19 & $1,012-1,031$ \\
\hline Sand and shale streaks & 67 & $1,031-1,098$ \\
\hline Shale and sand streaks & 16 & $1,098-1,114$ \\
\hline Sand and sandy shale & 24 & $1,114-1,138$ \\
\hline Shale and sand streaks & 14 & $1,138-1,152$ \\
\hline Sand and shale & 43 & $1,152-1,195$ \\
\hline Shale & 18 & $1,195-1,213$ \\
\hline Shale and sand layers & 18 & $1,213-1,231$ \\
\hline Shale & 5 & $1,231-1,236$ \\
\hline Shale and sand streaks & 34 & $1,236-1,270$ \\
\hline Shale, sandy & 39 & $1,270-1,309$ \\
\hline Sand and sandy shale & 51 & $1,309-1,360$ \\
\hline Shale & 11 & $1,360-1,371$ \\
\hline Shale, sandy and sand & 28 & $1,371-1,399$ \\
\hline Sand and shale streaks & 27 & $1,399-1,426$ \\
\hline Shale & 26 & $1,426-1,452$ \\
\hline Sand & 35 & $1,452-1,487$ \\
\hline Shale, sandy & 13 & $1,487-1,500$ \\
\hline
\end{tabular}

Well JY-65-28-209

Owner: Fort Bend County Water Control and Improvement District 2

Driller: Alsay-Pippin Corp.

\begin{tabular}{|c|c|c|}
\hline Description & $\begin{array}{c}\text { Thick- } \\
\text { ness } \\
\text { (feet) }\end{array}$ & $\begin{array}{c}\text { Depth } \\
\text { interval } \\
\text { (feet) }\end{array}$ \\
\hline Clay, sandy, red & 30 & $0-$ \\
\hline Sand & 33 & $30-$ \\
\hline Clay, red & 127 & $63-190$ \\
\hline Sand, $\tan$ & 146 & $190-336$ \\
\hline Shale, gray & 16 & $336-\quad 352$ \\
\hline Sand, white and tan & 23 & $352-\quad 375$ \\
\hline Shale, gray & 85 & $375-\quad 460$ \\
\hline Sand, $\tan$ & 25 & $460-\quad 485$ \\
\hline Shale, gray & 70 & $485-555$ \\
\hline Sand, tan & 29 & $555-584$ \\
\hline Shale, gray & 14 & $584-598$ \\
\hline Sand and shale breaks & 174 & $598-772$ \\
\hline Shale, gray & 28 & $772-800$ \\
\hline Sand, $\tan$ & 64 & $800-864$ \\
\hline Shale, gray & 46 & $864-910$ \\
\hline Sand and shale breaks & 149 & $910-1,059$ \\
\hline Shale, gray & 41 & $1,059-1,100$ \\
\hline Sand, $\tan$ & 86 & $1,100-1,186$ \\
\hline Shale, gray & 20 & $1,186-1,206$ \\
\hline Sand, $\tan$ & 14 & $1,206-1,220$ \\
\hline Shale, gray & 67 & $1,220-1,287$ \\
\hline Sand, $\tan$ & 17 & $1,287-1,304$ \\
\hline Shale, gray & 41 & $1,304-1,345$ \\
\hline Sand, $\tan$ & 81 & $1,345-1,426$ \\
\hline Shale, gray & 138 & $1,426-1,564$ \\
\hline Sand, $\tan$ & 20 & $1,564-1,584$ \\
\hline Shale, gray & 48 & $1,584-1,632$ \\
\hline Sand, $\tan$ & 42 & $1,632-1,674$ \\
\hline Shale, gray & 33 & $1,674-1,707$ \\
\hline
\end{tabular}


Well JY-65-28-210

Owner: First Colony Municipal Utility District 9, Hell No.

Driller: Layne-Texas Co.

\begin{tabular}{|c|c|c|}
\hline Description & $\begin{array}{c}\text { Thick- } \\
\text { ness } \\
\text { (feet) }\end{array}$ & $\begin{array}{c}\text { Depth } \\
\text { interval } \\
\text { (feet) }\end{array}$ \\
\hline Surface & 2 & $0-2$ \\
\hline Clay and sandy clay & 8 & $2-10$ \\
\hline Clay & 12 & $10-22$ \\
\hline Sand, sandy clay and clay streaks & 9 & 22- 31 \\
\hline Clay and sandy clay streaks & 3 & $31-34$ \\
\hline Clay & 1 & $34-35$ \\
\hline Sand, clay and gravel & 21 & 35- 56 \\
\hline Clay and gravel & 4 & 56- 60 \\
\hline Clay and sandy clay streaks & 6 & $60-66$ \\
\hline Sand and gravel & 11 & $66-77$ \\
\hline Clay & 17 & $77-94$ \\
\hline Sand and gravel & 15 & $94-109$ \\
\hline Clay & 3 & $109-112$ \\
\hline Sand and clay streaks & 38 & $112-150$ \\
\hline Clay & 6 & $150-156$ \\
\hline Sand, gravel and clay streaks & 28 & $156-184$ \\
\hline Clay & 1 & $184-185$ \\
\hline Clay and sandy clay streaks & 10 & $185-195$ \\
\hline Clay & 5 & $195-200$ \\
\hline Clay, sandy clay and sand streaks & 11 & $200-211$ \\
\hline Sand, gravel and clay streaks & 7 & $211-218$ \\
\hline Sand and gravel & 6 & $218-224$ \\
\hline Clay & 7 & $224-231$ \\
\hline Sand and clay streaks & 31 & $231-262$ \\
\hline Clay & 13 & $262-275$ \\
\hline Sand and clay streaks & 11 & $275-286$ \\
\hline Clay & 15 & $286-301$ \\
\hline Clay and sandy clay streaks & 8 & $301-309$ \\
\hline Clay & 7 & $309-316$ \\
\hline Sand & 12 & $316-328$ \\
\hline Sand and clay streaks & 38 & $328-366$ \\
\hline Clay & 13 & $366-379$ \\
\hline Sand and clay streaks & 13 & $379-392$ \\
\hline Clay & 3 & $392-395$ \\
\hline
\end{tabular}

Well JY-65-28-210--Cont inued

Sand 3

$395-398$

Clay

Sand and clay streaks

$398-400$

400-412

Clay

$412-420$

Sand and clay streaks

$420-436$

Clay

Clay and sand streaks

436-472

Clay 24

472-475

Clay, gravel and sand

475-499

499-509

Clay

$509-529$

Clay and sand streaks, cemented 25

529-554

Sand and clay streaks, cemented

$554-606$

Clay and sand streaks, cemented

$606-616$

Clay

616-627

Sand, gravel and cemented sand

$627-642$

Clay

Sand, clay and gravel streaks

$642-649$

Clay, lime and sandy clay streaks

649-663

Sand, lime and clay streaks 17

$663-678$

$17 \quad 678-695$

Lime, hard

695-696

Clay, I ime and rock

696-700

Sand, clay and lime streaks $\quad 15$

Clay and lime streaks

$700-715$

Sand, clay, shale and lime streaks 12

715-719

$719-731$

Clay and sandy clay 5

$731-736$

Sand, gravel, lime and clay streaks 40

736- 776

Clay, sandy clay and sand streaks $32 \quad 776-808$

Sand

$808-810$

Clay and shale streaks $\quad 17$

810-827

Sand, fine gravel and shale streaks 32

827-859

Clay $\quad 27$

859-886

Sand and clay streaks

$886-936$

$\mathrm{Clay}$ and sand streaks

936-942

Clay

$942-948$

Sand and clay 18

$948-966$

Clay

$966-977$

Sand

$977-984$ 
Well JY-65-28-210--Cont inued

\begin{tabular}{|c|c|c|}
\hline Clay & 2 & $984-986$ \\
\hline Sand & 6 & $986-$ \\
\hline Clay and lime streaks & 3 & $992-$ \\
\hline Sand and clay streaks & 7 & $995-1, \mathrm{C}$ \\
\hline Clay, lime streaks and sand & 7 & $1,002-1,0$ \\
\hline Sand and clay & 5 & $1,009-1,0$ \\
\hline Sand, clay and lime streaks & 18 & $1,014-1,0$ \\
\hline Sand and clay & 9 & $1,032-1,0$ \\
\hline Clay & 11 & $1,041-1,05$ \\
\hline Sand and clay streaks & 18 & $1,052-1,07$ \\
\hline $\mathrm{Clay}$ & 8 & $1,070-1,07$ \\
\hline Sand and clay streaks & 5 & $1,078-1,08$ \\
\hline Clay & 22 & $1,083-1,10$ \\
\hline $\mathrm{Clay}$, sandy clay and sand streaks & 21 & $1,105-1,12$ \\
\hline Clay & 5 & $1,126-1,13$ \\
\hline $\begin{array}{l}\text { Clay, sand and shale streaks and } \\
\text { little gravel }\end{array}$ & 43 & $1,131-1$, \\
\hline Sand, clay streaks, lime and gravel & 20 & $1,174-1,19$ \\
\hline $\mathrm{Clay}$, sand streaks and sandy clay & 10 & $1,194-1,20$ \\
\hline Clay & 97 & $1,204-1,30$ \\
\hline Clay, soft & 9 & $1,301-1,3$ \\
\hline Clay & 7 & $1,310-1,3$ \\
\hline Clay, soft & 31 & $1,317-1,3$ \\
\hline Clay & 30 & $1,348-1,37$ \\
\hline Clay and few shale streaks & 18 & $1,378-1,3$ \\
\hline $\mathrm{Clay}$, sandy and clay & 12 & $1,396-1,40$ \\
\hline Shale and sandy shale & 4 & $1,408-1,4$ \\
\hline Shale, sandy and shale & 6 & $1,412-1,41$ \\
\hline Shale, sandy, shale and sand streaks & 42 & $1,418-1$ \\
\hline Clay and shale & 40 & 1,460 \\
\hline
\end{tabular}

Well JY-65-28-211

Owner: Fort Bend County Municipal Utility District 42, Well No. 1

Driller: Alsay Incorporated

\begin{tabular}{lcrr}
\hline \multicolumn{1}{c}{ Description } & $\begin{array}{c}\text { Thick- } \\
\text { ness } \\
\text { (feet) }\end{array}$ & $\begin{array}{c}\text { Depth } \\
\text { interval } \\
\text { (feet) }\end{array}$ \\
\hline Clay, red and brown & 22 & $0-$ & 22 \\
Sand & 31 & $22-$ & 53 \\
Sand and gravel & 77 & $53-$ & 130
\end{tabular}

Well JY-65-28-211--Cont inued

\begin{tabular}{|c|c|c|}
\hline Clay, red & 11 & $130-141$ \\
\hline Sand & 29 & $141-$ \\
\hline Gravel with sand & 108 & $170-27$ \\
\hline clay, white & 6 & $278-\quad 28$ \\
\hline Sand & 97 & $284-38$ \\
\hline clay, gray & 17 & $381-39$ \\
\hline Sand & 8 & 398- \\
\hline Shale & 6 & 406- 41 \\
\hline Sand & 10 & $412-\quad 422$ \\
\hline Shale with small amount of gray clay & 20 & $422-44$ \\
\hline Sand & 46 & $442-48$ \\
\hline Clay, white & 7 & 488- \\
\hline Sand and gravel & 33 & $495-\quad 528$ \\
\hline Clay, brown and white & 35 & $528-$ \\
\hline Sand and gravel & 57 & $563-620$ \\
\hline Clay, white & 8 & $620-62$ \\
\hline Gravel with small amount of sand & 68 & $628-696$ \\
\hline Sand with white clay & 87 & $696-78$ \\
\hline Sand with white clay streaks & 58 & $783-84$ \\
\hline Sand & 49 & $841-89$ \\
\hline Sand with trace of white clay & 74 & $890-96$ \\
\hline Sand & 106 & $964-1,070$ \\
\hline Gravel and small streaks of shale & 21 & $1,070-1,09$ \\
\hline Sand & 27 & $1,091-1,118$ \\
\hline Clay, blue and shale & 25 & $1,118-1,143$ \\
\hline Sand & 76 & $1,143-1,219$ \\
\hline Shale and some clay & 28 & $1,219-1,247$ \\
\hline Gravell and sand & 33 & $1,247-1,280$ \\
\hline Shale and some clay & 28 & $1,280-1,247$ \\
\hline Clay and some shale & 44 & $1,247-1,324$ \\
\hline Sand & 10 & $1,324-1,334$ \\
\hline Clay, brown and shale & 88 & $1,334-1,422$ \\
\hline Sand & 51 & $1,422-1,473$ \\
\hline Clay, white with brown clay & 27 & $1,473-1,5$ \\
\hline
\end{tabular}


Well JY-65-28-213

Owner: 5th Street Grocery

Driller: Geophysical Drilling, Inc.

\begin{tabular}{lcc}
\hline \multicolumn{1}{c}{ Description } & $\begin{array}{c}\text { Thick- } \\
\text { ness } \\
\text { (feet) }\end{array}$ & $\begin{array}{c}\text { Depth } \\
\text { interval } \\
\text { (feet) }\end{array}$ \\
\hline Sand & 20 & $0-20$ \\
Sand and clay & 40 & $20-60$ \\
Clay and sand & 20 & $60-80$ \\
Sand & 20 & $80-100$ \\
Sand and red clay & 20 & $100-120$ \\
Clay & 100 & $120-220$ \\
Clay and sand & 20 & $220-240$ \\
Clay & 94 & $240-334$ \\
Sand & 18 & $334-352$
\end{tabular}

Hell JY-65-28-309

Owner: Blue Ridge Municipal Utility District, Chasewood, Well No. 1

Driller: Layne-Texas Co.

\begin{tabular}{|c|c|c|}
\hline Description & $\begin{array}{l}\text { Thick- } \\
\text { ness } \\
\text { (feet) }\end{array}$ & $\begin{array}{c}\text { Depth } \\
\text { interval } \\
\text { (feet) }\end{array}$ \\
\hline Topsoil & 3 & $0-3$ \\
\hline $\mathrm{Clay}$ and sand & 43 & 3- 46 \\
\hline Sand and clay streaks & 84 & $46-130$ \\
\hline $\mathrm{Clay}$ and sand streaks & 117 & $130-247$ \\
\hline Sand and clay streaks & 37 & $247-284$ \\
\hline Clay, sandy and sand streaks & 16 & $284-300$ \\
\hline Sand & 20 & $300-320$ \\
\hline Clay, sandy and clay & 34 & $320-354$ \\
\hline Sand and sandy clay & 58 & $354-412$ \\
\hline Shale & 19 & $412-431$ \\
\hline Sand & 6 & $431-437$ \\
\hline Shale and sandy shale & 15 & $437-452$ \\
\hline Shale, sandy and sand streaks & 36 & $452-488$ \\
\hline Sand and shale streaks & 22 & $488-510$ \\
\hline Shale & 38 & $510-548$ \\
\hline Sand and shale streaks & 23 & $548-571$ \\
\hline Shale, sandy & 9 & $571-580$ \\
\hline Sand and hard layers & 56 & $580-636$ \\
\hline Shale & 34 & $636-670$ \\
\hline Sand & 14 & $670-684$ \\
\hline
\end{tabular}

Well JY-65-28-309--Cont inued

$\begin{array}{lrrr}\text { Shale } & 17 & 684- & 701 \\ \text { Sand } & 39 & 701-740 \\ \text { Shale } & 7 & 740-747 \\ \text { Sand, coarse and gravel, fine } & 51 & 747-798 \\ \text { Shale } & 16 & 798-814 \\ \text { Sand } & 29 & 814-843 \\ \text { Shale } & 6 & 843-849 \\ \text { Shale and sand breaks } & 38 & 849-887 \\ \text { Sand and sandy shale and shale breaks } & 43 & 887-930 \\ \text { Sand and shale breaks, few } & 72 & 930-1,002 \\ \text { Sand and lime layers, hard } & 18 & 1,002-1,020 \\ \text { Shale } & 22 & 1,020-1,042 \\ \text { Shale, sandy and sand } & 88 & 1,042-1,130 \\ \text { Sand and shale breaks } & 85 & 1,130-1,215 \\ \text { Shale and sand breaks } & 35 & 1,215-1,250\end{array}$

Well JY-65-28-310

Owner: Willowisp Country Club

Driller: Bussell and Son, Inc.

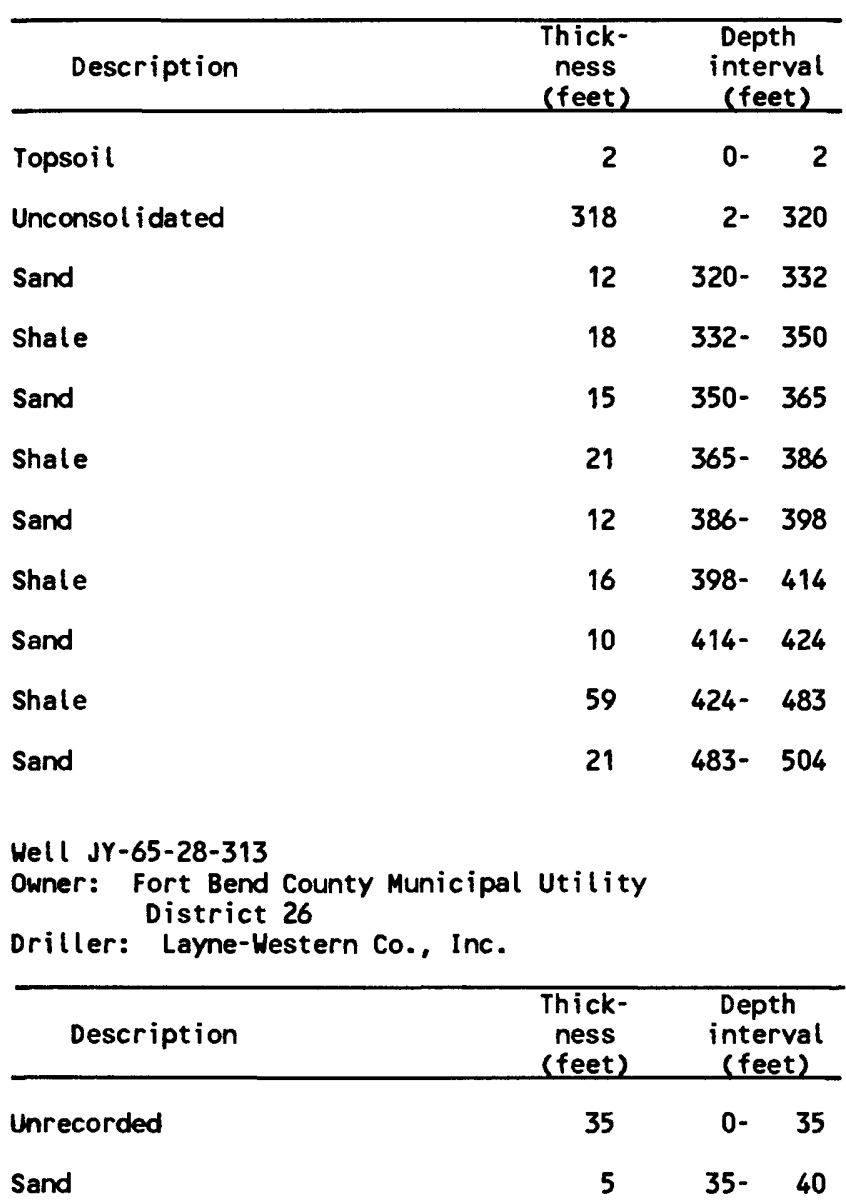


Well JY-65-28-313--Cont inued

Clay

Sand

Clay

Sand

Sand and clay streaks

Sand

Clay

Sand

Clay

Sand

Clay and sand streaks

Sand and clay streaks

Clay

Sand

Clay and sand streaks

Sand

Sand and clay streaks

Sand

Clay

Sand and clay

Clay and sand

Sand

Clay

Sand

Clay and sand streaks

Sand

Clay

Clay and sand streaks

Sand

Clay and sand

Sand

Sand and clay streaks

Clay

Sand with clay streaks

Sand

Sand and clay streaks

Sand
Well JY-65-28-313--Cont inued

$\begin{array}{rrl}3 & 40-43 & \text { Clay } \\ 12 & 43-55 & \text { Sand and clay streaks } \\ 8 & 55-63 & \text { Sand and hard streaks } \\ 20 & 63-83 & \text { Clay } \\ 19 & 83-102 & \text { Sand } \\ 20 & 102-122 & \text { Clay and sand streaks } \\ 9 & 122-131 & \text { Sand and hard streaks } \\ 18 & 131-149 & \text { Sand and clay streaks } \\ 3 & 149-152 & \text { Sand } \\ 18 & 152-170 & \text { Clay and sand with hard streaks } \\ 5 & 170-175 & \text { Clay } \\ 7 & 175-182 & \text { Sand }\end{array}$

$\begin{array}{lll}2 & 182-184 & \text { Clay } \\ 3 & 184-187 & \text { Sand }\end{array}$

6 187-193 Clay

16 193-209 Sand and clay streaks

$6 \quad 209-215$

Clay

Sand

Clay

Sand

$9 \quad 240-249$

$31 \quad 249-280$

$19 \quad 280-299$

11

299-310

$310-368$

Clay and sand streaks

Sand and clay streaks

Sand

Clay

Sand

Shale

Sand

Shale with sand streaks

435-465

30

465-498

498-543

Sand

Shale and limestone

Shale and sand streaks

Sand

Shate

14 591-605

$25 \quad 605-630$

Sand

$11 \quad 630-641$

Shale with sand streaks

641-653

Sand and shale
4 669- 673

18 673- 691

9 691- 700

$15700-715$

$2 \quad 715-717$

$6 \quad 717-723$

6 723- 729

17 729- 746

7 746- 753

8 753- 761

9 761- 770

$6770-776$

$3776-779$

$21 \quad 779-800$

$10 \quad 800-810$

$40 \quad 810-850$

13 850- 863

7 863- 870

$3 \quad 870-873$

24 873- 897

15 897- 912

9 912- 921

$29 \quad 921-950$

$14950-964$

$81964-1,045$

$61,045-1,051$

$37 \quad 1,051-1,088$

$31 \quad 1,088-1,119$

$15 \quad 1,119-1,134$

$10 \quad 1,134-1,144$

$61,144-1,150$

$221,150-1,172$

$8 \quad 1,172-1,180$

$531,180-1,233$

$20 \quad 1,233-1,253$

$47 \quad 1,253-1,300$

$16 \quad 653-669$ 
Hell JY-65-28-314

Owner: Fort Bend County Municipal Utility District 26

Driller: Bussell and Son, Inc.

\begin{tabular}{lcc}
\hline Description & $\begin{array}{c}\text { Thick- } \\
\text { ness } \\
\text { (feet) }\end{array}$ & $\begin{array}{c}\text { Depth } \\
\text { interval } \\
\text { (feet) }\end{array}$ \\
\hline Unconsol idated & 320 & $0-320$ \\
Sand, production & 83 & $320-403$
\end{tabular}

Hell JY-65-28-317

Owner: Willowisp Country Club

Driller: Bussell and Son, Inc.

\begin{tabular}{|c|c|c|}
\hline Description & $\begin{array}{c}\text { Thick- } \\
\text { ness } \\
\text { (feet) }\end{array}$ & $\begin{array}{c}\text { Depth } \\
\text { interval } \\
\text { (feet) }\end{array}$ \\
\hline Clay & 25 & $0-25$ \\
\hline Sand & 19 & $25-44$ \\
\hline Clay & 18 & 44- 62 \\
\hline Sand & 5 & $62-67$ \\
\hline Rock and sand & 77 & $67-144$ \\
\hline Clay & 48 & $144-192$ \\
\hline Sand & 18 & $192-210$ \\
\hline Clay & 102 & $210-312$ \\
\hline Sand & 4 & $312-316$ \\
\hline Clay & 10 & $316-326$ \\
\hline Sand & 11 & $326-337$ \\
\hline $\mathrm{Clay}$ & 12 & $337-349$ \\
\hline Sand & 16 & $349-365$ \\
\hline Clay & 20 & $365-385$ \\
\hline Sand & 17 & $385-402$ \\
\hline Clay & 8 & $402-410$ \\
\hline Sand & 20 & $410-430$ \\
\hline Clay & 50 & $430-480$ \\
\hline Sand & 25 & $480-505$ \\
\hline
\end{tabular}

Well JY-65-28-504

Owner: R. T. Herrin

Driller: American Drilling Co.

\begin{tabular}{lcc}
\hline \multicolumn{1}{c}{ Description } & $\begin{array}{c}\text { Thick- } \\
\text { ness } \\
\text { (feet) }\end{array}$ & $\begin{array}{c}\text { Depth } \\
\text { interval } \\
\text { (feet) }\end{array}$ \\
\hline Surface & 15 & $0-15$ \\
Sand, red & 6 & $15-21$ \\
Clay, red & 59 & $21-80$ \\
Sand, coarse & 30 & $80-110$
\end{tabular}

Hell JY-65-28-505

Owner: Thunderbird Utility District

Driller: Texas Water Hells, Inc.

\begin{tabular}{|c|c|c|c|}
\hline Description & $\begin{array}{c}\text { Thick- } \\
\text { ness } \\
\text { (feet) }\end{array}$ & $\begin{array}{r}\text { Dep } \\
\text { inte } \\
\text { Cfe } \\
\end{array}$ & $\begin{array}{l}\text { th } \\
\text { irval } \\
\text { tet) }\end{array}$ \\
\hline Clay and sand & 120 & 0- & 120 \\
\hline Sand, fine grain & 50 & $120-$ & 170 \\
\hline Shale, sandy & 70 & $170-$ & 240 \\
\hline Shale & 40 & $240-$ & 280 \\
\hline Shale and sand & 40 & $280-$ & 320 \\
\hline Shale, sandy & 50 & $320-$ & 370 \\
\hline Sand, with shale streaks & 325 & $370-$ & 695 \\
\hline Lime streaks & 35 & $695-$ & 730 \\
\hline \multirow{3}{*}{\multicolumn{4}{|c|}{$\begin{array}{l}\text { Sand, with streaks of fine gravel } \\
\text { or coarse sand } \\
\text { Shale, sticky } \\
\text { Hell JY-65-28-506 } \\
\text { Owner: Quail Valley Utility District, } \\
\text { Driller: Lell No. 1 }\end{array}$}} \\
\hline & & & \\
\hline & & & \\
\hline Description & $\begin{array}{c}\text { Thick- } \\
\text { ness } \\
\text { (feet) }\end{array}$ & \multicolumn{2}{|c|}{$\begin{array}{c}\text { Depth } \\
\text { interval } \\
\text { (feet) }\end{array}$} \\
\hline Topsoil & 4 & $0-$ & 4 \\
\hline Shale, brown & 56 & 4- & 60 \\
\hline Sand, coarse & 70 & $60-$ & 130 \\
\hline Shale, brown, hard sticky & 53 & $130-$ & 183 \\
\hline Sand and gravel & 65 & $183-$ & 248 \\
\hline Shale, hard & 114 & $248-$ & 362 \\
\hline Sand & 15 & $362-$ & 377 \\
\hline Shale & 35 & $377-$ & 412 \\
\hline Shale, sandy and sand & 32 & 412- & 444 \\
\hline Shale & 43 & 444- & 487 \\
\hline Sand and shale breaks & 32 & $487-$ & 519 \\
\hline Shale and sandy shale & 13 & 519- & 532 \\
\hline Sand and sandy shale & 17 & $532-$ & 549 \\
\hline Shale & 41 & 549- & 590 \\
\hline Sand, broken & 27 & $590-$ & 617 \\
\hline Shale & 12 & $617-$ & 629 \\
\hline Sand and shale breaks & 31 & $629-$ & 660 \\
\hline Shale, hard & 30 & $660-$ & 690 \\
\hline Sand & 6 & $690-$ & 696 \\
\hline
\end{tabular}


Table 3.--Drillers' logs of selected wells in Fort Bend County, 1969-87--Cont inued

Hell JY-65-28-506--Cont inued

Sand, coarse
Shale, blue
Sand, coarse
Shale, blue
Sand, coarse and gravel
Shale
Sand
Shale
Sand and shale streaks
Sand
Shale
Sand, broken
Shale
Sand and hard streaks
Shale and sand streaks
Sand and sand shale
Shale and sand streaks
Shale, sticky
Sand, broken
Shate

Hell JY-65-28-507

Owner: Thunderbird Utility District, Hell No. 3

Driller: Layne-Texas Co.

Description
Sand
Clay
Sand and clay streaks
Shale
Sand
Shale and sand streaks
Sand and shạle streaks
Shale and sand streaks
Sand and sandy shale
Shale and sand streaks
Sand
Shale and sand streaks

\begin{tabular}{rrr}
25 & $696-721$ \\
26 & $721-747$ \\
82 & $747-829$ \\
25 & $829-854$ \\
44 & $854-898$ \\
24 & $898-922$ \\
13 & $922-935$ \\
8 & $935-943$ \\
10 & $943-953$ \\
39 & $953-992$ \\
20 & $992-1,012$ \\
64 & $1,012-1,076$ \\
\hline 6 & $1,076-1,082$ \\
\hline 21 & $1,082-1,103$ \\
11 & $1,103-1,114$ \\
21 & $1,114-1,135$ \\
17 & $1,135-1,152$ \\
11 & $1,152-1,163$ \\
27 & $1,163-1,190$ \\
10 & $1,190-1,200$ \\
\hline 34
\end{tabular}

$-1,200$
Well JY-65-28-507--Cont inued

Sand 35

17

633- 668

Shale

Sand

$47 \quad 685-732$

Shale

24

$732-756$

Sand

Shale

Sand

28

756- 784

$11 \quad 784-795$

33

795- 828

Sand and shale streaks

53

828- 881

Shale

31

$881-912$

Sand

20

912- 932

Shale and sand streaks

28

932- 960

Sand

$80960-1,040$

Shale and sand streaks

$40 \quad 1,040-1,080$

Sand

$10 \quad 1,080-1,090$

Shale and sand streaks

$60 \quad 1,090-1,150$

Sand and shale streaks

$621,150-1,212$

Well JY-65-28-508

Owner: Quail Valley Utility District, Well No. 2

Driller: Layne-Western Co., Inc.

\begin{tabular}{lrrr}
\hline \multicolumn{1}{c}{ Description } & $\begin{array}{c}\text { Thick- } \\
\text { ness } \\
\text { (feet) }\end{array}$ & $\begin{array}{c}\text { Depth } \\
\text { interval } \\
\text { (feet) }\end{array}$ \\
\hline Clay & 4 & $0-$ & 4 \\
Sand, gravel and streaks & 60 & $4-$ & 64 \\
Clay, sandy & 66 & $64-$ & 130 \\
Clay & 11 & $130-$ & 141 \\
Sand & 11 & $141-$ & 152 \\
Clay & 33 & $152-$ & 185 \\
Sand & 66 & $185-$ & 251 \\
Clay & 116 & $251-367$ \\
Sand & 21 & $367-$ & 388 \\
Clay & 4 & $388-$ & 392 \\
Sand & 8 & $392-$ & 400 \\
Clay & 29 & $400-$ & 429 \\
Sand & 23 & $429-$ & 452 \\
Clay & $6505-$ & 486
\end{tabular}


Well JY-65-28-508--Cont inued

\begin{tabular}{|c|c|c|}
\hline Sand & 41 & $486-\quad 527$ \\
\hline Clay & 170 & $527-697$ \\
\hline Sand & 17 & $697-\quad 714$ \\
\hline Sand, stone & 1 & $714-715$ \\
\hline Sand & 14 & $715-729$ \\
\hline Clay & 22 & $729-\quad 751$ \\
\hline Sand & 15 & $751-766$ \\
\hline Rock & 3 & $766-769$ \\
\hline Sand & 4 & $769-773$ \\
\hline clay & 19 & $773-792$ \\
\hline Sand, gravel & 206 & $792-998$ \\
\hline Clay & 4 & $998-1,002$ \\
\hline Rock & 3 & $1,002-1,005$ \\
\hline Sand & 155 & $1,005-1,160$ \\
\hline Clay & 8 & $1,160-1,168$ \\
\hline Sand & 30 & $1,168-1,198$ \\
\hline clay & 17 & $1,198-1,215$ \\
\hline Sand & 93 & $1,215-1,308$ \\
\hline Clay & 5 & $1,308-1,313$ \\
\hline Unrecorded & 7 & $1,313-1,320$ \\
\hline
\end{tabular}

Hell JY-65-28-509

Owner: Palmer Plantation Municipal Utility District 1, Hell No. 1

Driller: Layne-Texas Co.

\begin{tabular}{lccc}
\hline \multicolumn{1}{c}{ Description } & $\begin{array}{c}\text { Thick- } \\
\text { ness } \\
\text { (feet) }\end{array}$ & $\begin{array}{c}\text { Depth } \\
\text { interval } \\
\text { (feet) }\end{array}$ \\
\hline Dirt & 13 & $0-$ & 13 \\
Sand & 67 & $13-$ & 80 \\
Clay & 20 & $80-$ & 100 \\
Sand & 24 & $100-$ & 124 \\
Clay & 12 & $124-$ & 136 \\
Sand and clay & 45 & $136-$ & 181 \\
Clay & 24 & $181-$ & 205 \\
Sand & 43 & $205-$ & 248 \\
Shale & 8 & $248-$ & 256 \\
Sand & 21 & $256-277$ \\
Shale and sand streaks & 66 & $277-343$ \\
Shale & 39 & $343-$ & 382
\end{tabular}

Well JY-65-28-509--Continued

\begin{tabular}{|c|c|c|}
\hline Sand & 26 & $382-408$ \\
\hline Shale & 40 & 408- $\quad 448$ \\
\hline Sand and shale streaks & 11 & $448-459$ \\
\hline Shale & 51 & $459-\quad 510$ \\
\hline Sand and shale streaks & 22 & $510-532$ \\
\hline Shale & 12 & $532-544$ \\
\hline Shale and sand streaks & 41 & $544-585$ \\
\hline Shale & 17 & $585-602$ \\
\hline Sand and shale streaks & 33 & $602-635$ \\
\hline Shale & 9 & $635-644$ \\
\hline Sand and few shale streaks & 31 & $644-675$ \\
\hline Shale & 32 & $675-707$ \\
\hline Clay and sandy clay & 7 & $707-\quad 714$ \\
\hline Shale and clay & 28 & $714-742$ \\
\hline Shale and sand streaks & 10 & $742-752$ \\
\hline Shale & 15 & $752-767$ \\
\hline Sand and clay streaks & 9 & $767-776$ \\
\hline Shale & 22 & $776-798$ \\
\hline clay, sandy clay and shale & 62 & $798-860$ \\
\hline Sand and clay & 25 & $860-885$ \\
\hline Shale & 29 & $885-914$ \\
\hline Clay and sandy clay & 32 & $914-946$ \\
\hline Shale and clay & 35 & $946-981$ \\
\hline Shale, sand and clay & 29 & $981-1,010$ \\
\hline Sand and clay streaks & 25 & $1,010-1,035$ \\
\hline Shale and lime streaks & 5 & $1,035-1,040$ \\
\hline Sand and clay & 21 & $1,040-1,061$ \\
\hline Clay, sandy and lime streaks & 13 & $1,061-1,074$ \\
\hline Shale and lime streaks & 3 & $1,074-1,077$ \\
\hline Clay and sandy streaks & 15 & $1,077-1,092$ \\
\hline Lime and sandy streaks & 6 & $1,092-1,098$ \\
\hline Lime & 7 & $1,098-1,105$ \\
\hline Shale & 34 & $1,105-1,139$ \\
\hline Sand & 28 & $1,139-1,167$ \\
\hline Shale and sand streaks & 7 & $1,167-1,174$ \\
\hline Shale, sandy & 17 & $1,174-1,191$ \\
\hline Sand & 8 & $1,191-1,199$ \\
\hline
\end{tabular}


Well JY-65-28-509--Cont inued

Clay, sandy

$91,199-1,208$

Clay and shale

$131,208-1,221$

Well JY-65-28-510

Owner: Fort Bend County Municipal Utility District 46, Well No. 1

Driller: Layne-Texas Co.

\begin{tabular}{|c|c|c|}
\hline Description & $\begin{array}{l}\text { Thick- } \\
\text { ness } \\
\text { (feet) }\end{array}$ & $\begin{array}{c}\text { Depth } \\
\text { interval } \\
\text { (feet) }\end{array}$ \\
\hline Clay, black and red & 24 & $0-$ \\
\hline Gravel & 11 & 24- \\
\hline Sand & 53 & 35- \\
\hline Wood & 7 & 88- \\
\hline Sand and clay streaks & 92 & 95- $\quad 187$ \\
\hline Sand & 25 & $187-212$ \\
\hline Sand with few clay streaks & 24 & $212-\quad 236$ \\
\hline Shale & 28 & $236-264$ \\
\hline Sand with shale streaks & 14 & $264-278$ \\
\hline Shale & 72 & $278-350$ \\
\hline Sand with shale breaks & 58 & $350-408$ \\
\hline Shale & 18 & $408-426$ \\
\hline Sand & 7 & 426- 433 \\
\hline Shale and lime streaks & 77 & 433- $\quad 510$ \\
\hline Shale & 20 & $510-530$ \\
\hline Sand & 67 & $530-597$ \\
\hline Shale & 25 & $597-622$ \\
\hline Sand & 67 & $622-689$ \\
\hline Shale & 31 & $689-720$ \\
\hline Sand and shale streaks & 98 & $720-818$ \\
\hline Shale & 47 & $818-865$ \\
\hline Sand and shale streaks & 59 & $865-924$ \\
\hline Sand & 34 & $924-958$ \\
\hline Sand and shale streaks & 97 & $958-1,055$ \\
\hline Sand and hard lime streaks & 22 & $1,055-1,077$ \\
\hline Shale & 4 & $1,077-1,081$ \\
\hline Sand and lime streaks & 31 & $1,081-1,112$ \\
\hline Shale & 41 & $1,112-1,153$ \\
\hline Shale, sandy and sand streaks & 31 & $1,153-1,184$ \\
\hline Sand & 46 & $1,184-1,230$ \\
\hline Shale and lime streaks & 6 & $1,230-1,236$ \\
\hline
\end{tabular}

Hell JY-65-28-603

Owner: Quail Valley Utility District, Well No. 2

Driller: Texas Water Wells, Inc.

\begin{tabular}{|c|c|c|}
\hline Description & $\begin{array}{c}\text { Thick- } \\
\text { ness } \\
\text { (feet) }\end{array}$ & $\begin{array}{c}\text { Depth } \\
\text { interval } \\
\text { (feet) }\end{array}$ \\
\hline Surface & 4 & $0-4$ \\
\hline Clay & 11 & $4-15$ \\
\hline Sand & 48 & $15-63$ \\
\hline Clay & 24 & $63-87$ \\
\hline Sand & 12 & $87-99$ \\
\hline $\mathrm{Clay}$ & 6 & $99-105$ \\
\hline Clay, sandy & 16 & $105-121$ \\
\hline Sand and gravel & 31 & $121-152$ \\
\hline Clay & 14 & $152-166$ \\
\hline Sand & 25 & $166-191$ \\
\hline Clay & 29 & $191-220$ \\
\hline Sand & 4 & $220-224$ \\
\hline Clay and sand streaks & 9 & $224-233$ \\
\hline clay & 30 & $233-263$ \\
\hline Sand & 15 & $263-278$ \\
\hline Clay & 42 & $278-320$ \\
\hline Sand & 11 & $320-331$ \\
\hline Clay and sandy streaks & 60 & $331-391$ \\
\hline Clay & 12 & $391-403$ \\
\hline Shale & 53 & $403-456$ \\
\hline Sand & 19 & $456-475$ \\
\hline Shale & 66 & $475-541$ \\
\hline Sand & 17 & $541-558$ \\
\hline Shale & 76 & $558-634$ \\
\hline Sand & 7 & $634-641$ \\
\hline Shale, sandy & 12 & $641-653$ \\
\hline Sand & 39 & $653-692$ \\
\hline Shale & 13 & $692-705$ \\
\hline Sand & 38 & $705-743$ \\
\hline Shale, sandy & 48 & $743-791$ \\
\hline Sand & 62 & $791-853$ \\
\hline Shale & 6 & $853-859$ \\
\hline Sand & 28 & $859-887$ \\
\hline Shale & 10 & $887-897$ \\
\hline
\end{tabular}


Well JY-65-28-603--Cont inued

Sand
Shale, sand
Sand
Shale
Sand
Shale
Sand
Shale
Sand
Shale, sandy
Well JY-65-28-605
Owner: Fort Bend County Municipal
Driller: Layne-Western Co., Inc.

\begin{tabular}{|c|c|c|c|}
\hline Description & $\begin{array}{c}\text { Thick- } \\
\text { ness } \\
\text { (feet) }\end{array}$ & $\begin{array}{l}\text { Dep } \\
\text { inte } \\
\text { (fe }\end{array}$ & $\begin{array}{l}\text { th } \\
\text { rval } \\
\text { et) }\end{array}$ \\
\hline Clay & 16 & $0-$ & 16 \\
\hline Sand & 10 & $16-$ & 26 \\
\hline Clay & 4 & 26- & 30 \\
\hline Sand & 37 & $30-$ & 67 \\
\hline Clay & 15 & $67-$ & 82 \\
\hline Sand & 94 & 82- & 176 \\
\hline Clay & 14 & 176- & 190 \\
\hline Shale, sandy & 20 & $190-$ & 210 \\
\hline Sand & 29 & $210-$ & 239 \\
\hline Shale & 94 & 239- & 333 \\
\hline Shale, sandy & 20 & 333- & 353 \\
\hline Shale & 47 & $353-$ & 400 \\
\hline Sand & 47 & $400-$ & 447 \\
\hline Shale & 46 & 447- & 493 \\
\hline Sand & 5 & 493- & 498 \\
\hline Shale & 7 & 498- & 505 \\
\hline Sand & 17 & $505-$ & 522 \\
\hline Shale & 28 & $522-$ & 550 \\
\hline Sand & 10 & $550-$ & 560 \\
\hline Shale & 27 & $560-$ & 587 \\
\hline Sand & 5 & $587-$ & 592 \\
\hline Shale, sandy & 36 & $592-$ & 628 \\
\hline Sand & 15 & 628- & 643 \\
\hline
\end{tabular}

Well JY-65-28-605--Continued

$\begin{array}{rrlllll}53 & 897-950 & \text { shale } & 57 & 643-700 \\ 20 & 950-970 & \text { sand } & 19 & 700-719 \\ 38 & 970-1,008 & \text { shale } & 3 & 719-722 \\ 26 & 1,008-1,034 & \text { sand } & 10 & 722-732 \\ 26 & 1,034-1,060 & \text { shale } & 19 & 732-751 \\ 2 & 1,060-1.062 & \text { sand } & 85 & 751-836 \\ 56 & 1,062-1,118 & \text { Shale } & 7 & 836-843 \\ 1 & 1,118-1,119 & \text { sand } & 19 & 843-862 \\ 6 & 1,119-1,125 & \text { shale } & 2 & 862-864 \\ 11 & 1,125-1,136 & \text { sand } & 129 & 864-993\end{array}$

Hell JY-65-28-606

Owner: Houston International Teleport, Incorporated

Driller: Raymond Water Wells

\begin{tabular}{lccc}
\hline \multicolumn{1}{c}{ Description } & $\begin{array}{c}\text { Thick- } \\
\text { ness } \\
\text { (feet) }\end{array}$ & $\begin{array}{c}\text { Depth } \\
\text { interval } \\
\text { (feet) }\end{array}$ \\
\hline Topsoil and black clay & 20 & $0-$ & 20 \\
Clay, gray & 40 & $20-$ & 60 \\
Sand & 20 & $60-$ & 80
\end{tabular}

Hell JY-65-28-702

Owner: Glen R. Shultz

Driller: Almeda Water Well Service

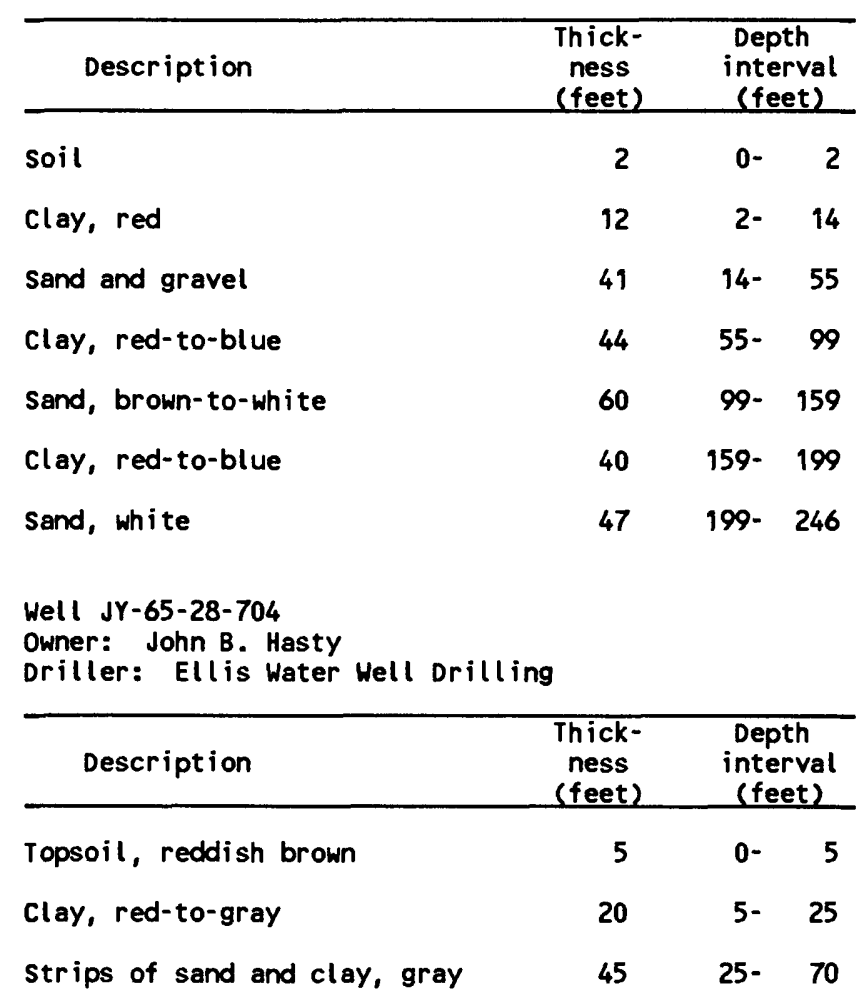


Well JY-65-28-704--Cont inued

clay, red-to-gray

Sand, fair gravel

$35 \quad 70-105$

Clay, red-to-gray

$35 \quad 105-140$

Sand, fair-to-coarse

$50 \quad 140-190$

Clay, gray

$22 \quad 190-212$

2 212-214

Sand, very coarse-to-gravel

$19 \quad 214-233$

Well JY-65-28-705

Owner: Robert C. Newton

Driller: Ellis Water Hell Drilling

\begin{tabular}{lcc}
\hline \multicolumn{1}{c}{ Description } & $\begin{array}{c}\text { Thick- } \\
\text { ness } \\
\text { (feet) }\end{array}$ & $\begin{array}{c}\text { Depth } \\
\text { interval } \\
\text { (feet) }\end{array}$ \\
\hline Topsoil, reddish brown & 6 & $0-6$ \\
Clay, red-to-gray & 24 & $6-30$ \\
Sand and gravel strips & 50 & $30-80$ \\
Clay, red & 20 & $80-100$ \\
Sand, fair coarse & 50 & $100-150$ \\
Clay, red-to-gray & 45 & $150-195$ \\
Sand, fair & 17 & $195-212$ \\
Clay, grayish & 3 & $212-215$ \\
Sand, extra coarse & 22 & $215-237$ \\
Uell JY-65-28-706 & & $230-250$ \\
Owner: Newberne \\
Driller: Ellis Water Well Drilling
\end{tabular}

Hell JY-65-28-707

Owner: Charles J. Shuman

Driller: Ellis Water Hell Drilling

\begin{tabular}{lcr}
\hline \multicolumn{1}{c}{ Description } & $\begin{array}{c}\text { Thick- } \\
\text { ness } \\
\text { (feet) }\end{array}$ & $\begin{array}{c}\text { Depth } \\
\text { interval } \\
\text { (feet) }\end{array}$ \\
\hline Topsoil, blackish brown & 2 & $0-2$ \\
Soil, red, sandy & 4 & $2-6$ \\
Clay, red and gray & 24 & $6-30$ \\
Sand, fair-to-gravel & 50 & $30-80$ \\
Clay, red and gray & 30 & $80-110$ \\
Sand and gravel strips & 50 & $110-160$ \\
Clay, reddish gray & 25 & $160-185$ \\
Sand, fair-to-coarse & 30 & $185-215$ \\
Clay, gray & 10 & $215-225$ \\
Sand and gravel strips & 25 & $225-250$ \\
Clay, reddish gray & & $250-270$ \\
Sand, fair-to-very coarse & 20 & $270-303$ \\
Well Jy-65-28-708 & & \\
Owner: Bill Cayan \\
Driller: Ellis Water Well Drilling
\end{tabular}

\begin{tabular}{|c|c|c|}
\hline Description & $\begin{array}{c}\text { Thick- } \\
\text { ness } \\
\text { (feet) }\end{array}$ & $\begin{array}{l}\text { Depth } \\
\text { interval } \\
\text { (feet) }\end{array}$ \\
\hline Topsoil, brownish & 5 & $0-5$ \\
\hline clay, red and gray & 21 & 5- 26 \\
\hline Sand, light brownish & 9 & $26-35$ \\
\hline Sand, quick & 35 & $35-70$ \\
\hline Clay, red-to-gray & 42 & $70-112$ \\
\hline Sand, fair coarse and gravel & 36 & $112-148$ \\
\hline clay, red-to-gray & 47 & $148-195$ \\
\hline Sand, fair coarse & 20 & $195-215$ \\
\hline clay, gray & 5 & $215-220$ \\
\hline Sand, coarse and gravel & 48 & $220-268$ \\
\hline \multicolumn{3}{|c|}{$\begin{array}{l}\text { Well Jy-65-28-709 } \\
\text { Owner: Drake Williams } \\
\text { Driller: Ellis Water Well Drilling }\end{array}$} \\
\hline Description & $\begin{array}{l}\text { Thick- } \\
\text { ness } \\
\text { (feet) }\end{array}$ & $\begin{array}{l}\text { Depth } \\
\text { interval } \\
\text { (feet) }\end{array}$ \\
\hline Topsoill, brownish red & 5 & $0-5$ \\
\hline Clay, red and gray & 30 & $5-35$ \\
\hline Sand and sand rock, strips & 55 & $35-90$ \\
\hline
\end{tabular}


Well JY-65-28-709--Continued

Clay, red-to-gray

$3090-120$

Sand and gravel, strips

$40 \quad 120-160$

Clay, red-to-gray

$35 \quad 160-195$

Sand and clay, strips

clay, red-to-gray, sticky

$45 \quad 195-240$

Sand, very coarse and gravel

$38 \quad 240-278$

$25 \quad 278-303$

Well JY-65-28-710

Owner: Peter Mellan

Driller: Ellis Water Well Drilling

\begin{tabular}{lcc}
\hline \multicolumn{1}{c}{ Description } & $\begin{array}{c}\text { Thick- } \\
\text { ness } \\
\text { (feet) }\end{array}$ & $\begin{array}{c}\text { Depth } \\
\text { interval } \\
\text { (feet) }\end{array}$ \\
\hline Topsoil, brownish red & 5 & $0-5$ \\
Clay, red-to-gray & 35 & $5-40$ \\
Sand and gravel strips & 45 & $40-85$ \\
Clay, red & 25 & $85-110$ \\
Sand and gravel & 45 & $110-155$ \\
Clay, red-to-gray & 30 & $155-185$ \\
Sand, fair coarse & 30 & $185-215$ \\
Clay, gray & 10 & $215-225$ \\
Sand, good coarse & 17 & $225-242$
\end{tabular}

Well JY-65-28-711

Owner: Arthur Kennedy

Driller: Ellis Water Well Drilling

\begin{tabular}{lcr}
\hline \multicolumn{1}{c}{ Description } & $\begin{array}{c}\text { Thick- } \\
\text { ness } \\
\text { (feet) }\end{array}$ & $\begin{array}{c}\text { Depth } \\
\text { interval } \\
\text { (feet) }\end{array}$ \\
\hline Topsoil, reddish brown & 6 & $0-6$ \\
Clay, red and gray & 27 & $6-33$ \\
Sand and gravel, strips & 57 & $33-90$ \\
Clay, red-to-gray & 20 & $90-110$ \\
Sand and gravel strips & 50 & $110-160$ \\
Clay, red and gray, sticky & 40 & $160-200$ \\
Sand, fair coarse & 10 & $200-210$ \\
Clay, gray & 11 & $210-221$ \\
Sand, very coarse and gravel & 22 & $221-243$
\end{tabular}

Well JY-65-28-808

Owner: Hurricane Steel Industries

Driller: Wellco Water Well Drilling Co.

\begin{tabular}{|c|c|c|}
\hline Description & $\begin{array}{l}\text { Thick- } \\
\text { ness } \\
\text { (feet) }\end{array}$ & $\begin{array}{c}\text { Depth } \\
\text { interval } \\
\text { (feet) }\end{array}$ \\
\hline Soil and clay & 23 & $0-23$ \\
\hline Sand & 17 & $23-40$ \\
\hline Sand and gravel & 20 & $40-60$ \\
\hline Gravel & 20 & $60-80$ \\
\hline Gravel & 20 & $80-100$ \\
\hline Clay & 20 & $100-120$ \\
\hline Sand, strips and clay & 20 & $120-140$ \\
\hline Clay & 20 & $140-160$ \\
\hline Sand & 20 & $160-180$ \\
\hline Clay and fine sand & 20 & $180-200$ \\
\hline Rock and clay & 20 & $200-220$ \\
\hline Clay & 20 & $220-240$ \\
\hline Sand and clay & 20 & $240-260$ \\
\hline $\mathrm{Clay}$ and rock & 20 & $260-280$ \\
\hline Clay & 20 & $280-300$ \\
\hline Rock and clay & 20 & $300-320$ \\
\hline Sand, coarse & 20 & $320-340$ \\
\hline Sand, fine & 20 & $340-360$ \\
\hline Clay & 20 & $360-380$ \\
\hline Rock and clay & 20 & $380-400$ \\
\hline Clay & 20 & $400-420$ \\
\hline Sand, fine & 20 & $420-440$ \\
\hline Clay, sand and shale & 20 & $440-460$ \\
\hline Shale, blue & 20 & $460-480$ \\
\hline Clay & 20 & $480-500$ \\
\hline Rock and clay & 20 & $500-520$ \\
\hline Sand, coarse & 40 & $520-560$ \\
\hline \multicolumn{3}{|c|}{$\begin{array}{l}\text { Well JY- } 65-28-902 \\
\text { Owner: Waterbrook } \\
\text { Driller: Almeda Water Well Service }\end{array}$} \\
\hline Description & $\begin{array}{c}\text { Thick- } \\
\text { ness } \\
\text { (feet) }\end{array}$ & $\begin{array}{c}\text { Depth } \\
\text { interval } \\
\text { (feet) }\end{array}$ \\
\hline Topsoil & 3 & $0-3$ \\
\hline Clay & 3 & 3- 6 \\
\hline Sand & 24 & $6-30$ \\
\hline
\end{tabular}


Well JY-65-28-902--Cont inued

\begin{tabular}{lcc} 
Clay & 3 & $30-33$ \\
Sand & 39 & $33-72$ \\
Clay & 18 & $72-90$ \\
Sand and rock & 1 & $90-91$ \\
Clay & 14 & $91-105$ \\
Well JY-65-28-903 & & \\
Owner: Bill Senior \\
Driller: Almeda Water Well Service & & \\
\hline & & \\
Description & Thick- & Depth \\
interval \\
(feet)
\end{tabular}

Well JY-65-29-109

Owner: City of Houston, Ridgemont, Well No. 2

Driller: Alsay-Texas Corporation

\begin{tabular}{lcc}
\hline \multicolumn{1}{c}{ Description } & $\begin{array}{c}\text { Thick- } \\
\text { ness } \\
\text { (feet) }\end{array}$ & $\begin{array}{c}\text { Depth } \\
\text { interval } \\
\text { (feet) }\end{array}$ \\
\hline Clay, black & 10 & $0-10$ \\
Clay, red & 8 & $10-18$ \\
Sand, red & 5 & $18-23$ \\
Clay, red & 5 & $23-28$ \\
Sand & 3 & $28-31$ \\
Clay, blue & 22 & $31-53$ \\
Sand, gray & 30 & $53-83$ \\
Clay, red and sand & 26 & $83-109$ \\
Clay, red and gravel & 26 & $109-135$ \\
Gravel & 30 & $135-165$ \\
Clay, sandy & 15 & $165-180$ \\
Clay with gravel & 18 & $180-198$ \\
Sand & 42 & $198-240$ \\
Clay, red & 15 & $240-255$ \\
Clay, sandy & 9 & $255-264$ \\
Clay & 14 & $264-278$
\end{tabular}

Well JY-65-29-109--Cont inued

Clay, sandy streaks

$197 \quad 278-475$

Clay, red

$50 \quad 475-525$

Sand and clay

$50 \quad 525-575$

Sand

575- 585

Clay, sandy

$585-595$

clay, red

595- 605

clay, red

Clay, red and sand

605- 615

Clay, red

615- 625

clay, red

Clay, red with gravel

$635-645$

Clay, red and blue

$645-655$

Sand

$655-665$

Sand with red clay streaks

$665-675$

675. 685

Sand

$685-695$

Sand with clay streaks

$695-715$

Sand

$715-725$

Sand and red clay

$725-735$

Sand with red clay streaks

$735-755$

Sand

$755-765$

Sand

765- 775

Clay, red

$775-795$

Sand with blue clay streaks

$795-890$

Clay, blue with sand streaks

Sand with blue clay streaks

Clay, blue with sand streaks

Sand

Clay, blue with sand streaks

clay, blue

Sand

Sand

Sand with red clay streaks

Sand with red clay streaks

Sand, white

Sand and blue clay streaks

Sand and clay streaks

890- 905

15

20

905- 925

39

925- 964

10

864- 974

$8974-982$

3

982- 985

$10 \quad 985-995$

$10 \quad 995-1,005$

$10 \quad 1,005-1,015$

$10 \quad 1,015-1,025$

$10 \quad 1,025-1,035$

$10 \quad 1,035-1,045$

$10 \quad 1,045-1,055$

clay, blue very hard

$10 \quad 1,055-1,065$ 
Well JY-65-29-109--Cont inued

Clay, hard and sand

Sand and blue shale

Sand and fine gravel

Sand

Sand, white, hard

Sand, white

Sand

Sand with blue clay streaks

Sand

Clay, light blue and sand

Sand with trace of gravel

Sand and blue clay

clay and shale

Sand

clay and shale

Sand, white and gravel

Clay

Well JY-65-29-209

Owner: City of Houston, Ridgemont, Hell No. 1

Driller: Layne-Texas Co.

\begin{tabular}{lccc}
\hline \multicolumn{1}{c}{ Description } & $\begin{array}{c}\text { Thick- } \\
\text { ness } \\
\text { (feet) }\end{array}$ & $\begin{array}{c}\text { Depth } \\
\text { interval } \\
\text { (feet) }\end{array}$ \\
\hline Clay & 27 & $0-$ & 27 \\
Clay, sandy & 26 & $27-$ & 53 \\
Sand & 20 & $53-$ & 73 \\
Sand and gravel & 98 & $73-$ & 171 \\
Clay and sand & 23 & $171-$ & 194 \\
Sand and clay, sandy & 22 & $194-$ & 216 \\
Sand & 16 & $216-$ & 232 \\
Clay & 53 & $232-$ & 285 \\
Sand & 25 & $285-$ & 310 \\
Shale, sandy & 16 & $310-$ & 326 \\
Sand, broken & 33 & $326-359$ \\
Shale & 17 & $359-$ & 376 \\
Sand and l ime streaks & 22 & $376-398$ \\
Shale & 29 & $398-427$ \\
Sand and lime streaks & 56 & $427-483$
\end{tabular}

$201,285-1,305$

$10 \quad 1,305-1,315$

$201,315-1,335$

$101,335-1,345$

$101,345-1,355$

$101,355-1,365$

$361,365-1,401$

$5 \quad 1,401-1,406$

$491,406-1,455$

$481,455-1,503$

$21,503-1,505$
Well JY-65-29-209--Cont inued

Shale and sand streaks 63

483- 546

Sand and gravel

$19 \quad 546-565$

Shale and sand streaks

$75 \quad 565-640$

Sand and shale, sandy

$38 \quad 640-678$

Shale and sand streaks

$67 \quad 678-745$

Shale

Sand, broken

Shale

Sand and shale

8

745- 753

Shale and sandy streaks

Sand

Shale and lime streaks

Rock

Shale

$118 \quad 753-871$

$7 \quad 871-878$

$20 \quad 878-898$

$50 \quad 898-948$

9 948- 957

$59 \quad 957-1,016$

$3 \quad 1,016-1,019$

$31 \quad 1,019-1,050$

Shale with streaks of sand and lime $27 \quad 1,050-1,077$

Sand, broken

$31 \quad 1,077-1,108$

Shale

Sand, broken

$5 \quad 1,108-1,113$

Shale

$331,113-1,146$

$191,146-1,165$

Sand

$13 \quad 1,165-1,178$

Shale

$13 \quad 1,178-1,191$

Shale, sandy and sand streaks

$591,191-1,250$

Well JY-65-29-515

Owner: Witco Chemical Corporation Driller: B. J. Swinehart Co.

\begin{tabular}{lcr}
\hline \multicolumn{1}{c}{ Description } & $\begin{array}{c}\text { Thick- } \\
\text { ness } \\
\text { (feet) }\end{array}$ & $\begin{array}{c}\text { Depth } \\
\text { interval } \\
\text { (feet) }\end{array}$ \\
\hline Unrecorded & 417 & $0-417$ \\
Clay & 23 & $417-440$ \\
Sand, fine & 22 & $440-462$ \\
Clay & 23 & $462-485$ \\
Sand, broken & 10 & $485-495$ \\
Clay & 29 & $495-524$ \\
Sand & 3 & $524-527$ \\
Clay & 52 & $527-579$ \\
Sand, good & 16 & $579-595$ \\
Clay & 23 & $595-618$
\end{tabular}


Well JY-65-29-516

Owner: Heat Exchangers, Inc.

Driller: Almeda Water Hell Service

\begin{tabular}{lrr}
\hline \multicolumn{1}{c}{ Description } & $\begin{array}{c}\text { Thick- } \\
\text { ness } \\
\text { (feet) }\end{array}$ & $\begin{array}{c}\text { Depth } \\
\text { interval } \\
\text { (feet) }\end{array}$ \\
\hline Soil & 2 & $0-2$ \\
Clay, white and blue & 34 & $2-36$ \\
Sand and gravel & 76 & $36-112$ \\
Clay, red & 8 & $112-120$ \\
Sand, red and fine & 5 & $120-125$ \\
Clay, red & 13 & $125-138$ \\
Sand, brown & 27 & $138-165$ \\
Clay, red & 23 & $165-188$ \\
Sand, brown & 20 & $188-208$ \\
Clay, blue & 6 & $108-214$ \\
Sand, white & 34 & $214-248$ \\
Clay, red and blue & 92 & $248-340$ \\
Sand, white & 25 & $340-365$
\end{tabular}

Hell JY-65-29-517

Owner: Witco Chemical Corporation

Driller: H\&H Water Well Drilling

\begin{tabular}{|c|c|c|}
\hline Description & $\begin{array}{l}\text { Thick- } \\
\text { ness } \\
\text { (feet) }\end{array}$ & $\begin{array}{c}\text { Depth } \\
\text { interval } \\
\text { (feet) }\end{array}$ \\
\hline Clay & - & - \\
\hline Sand & - & - \\
\hline Clay & - & - \\
\hline Sand & - & - \\
\hline Clay & - & 0.245 \\
\hline Sand & 10 & $245 \cdot 255$ \\
\hline Clay & 65 & $255-320$ \\
\hline Sand & 32 & $320-352$ \\
\hline Clay & 17 & $352-369$ \\
\hline Sand & 15 & $369-384$ \\
\hline Clay & 32 & $384-416$ \\
\hline Sand & 12 & $416-428$ \\
\hline Clay & 62 & $428-490$ \\
\hline Sand & 20 & $490-510$ \\
\hline Clay & 12 & $510-522$ \\
\hline Sand & 22 & $522-544$ \\
\hline Clay & 11 & $544-555$ \\
\hline
\end{tabular}

Well JY-65-29-517--Cont inued

Sand

Clay

Sand

Well JY-65-29-518

Owner: Hitco Chemical Corporation

Driller: Almeda Hater Hell Service

\begin{tabular}{lrr}
\hline \multicolumn{1}{c}{ Description } & $\begin{array}{c}\text { Thick- } \\
\text { ness } \\
\text { (feet) }\end{array}$ & $\begin{array}{c}\text { Depth } \\
\text { interval } \\
\text { (feet) }\end{array}$ \\
\hline Topsoil & 2 & $0-2$ \\
Clay, thite & 10 & $2-12$ \\
Sand, red & 9 & $12-21$ \\
Clay, red & 9 & $21-30$ \\
Sand, red & 4 & $30-34$ \\
Sand and gravel & 46 & $34-80$ \\
Clay, yellow & 67 & $80-147$ \\
Sand, tan & 6 & $147-153$ \\
Clay, red & 80 & $153-233$ \\
Sand ' & 6 & $233-239$ \\
Clay, gray & 39 & $239-278$ \\
Sand & 8 & $278-286$ \\
Clay, red & 63 & $286-349$ \\
Sand, white & 16 & $349-365$ \\
Clay & 2 & $365-367$ \\
Sand & 8 & $367-375$ \\
Clay & $375-379$
\end{tabular}

Hell JY-65-29-705

Owner: H. E. Gantenbien

Driller: Almeda Water Hell Service

\begin{tabular}{lcr}
\hline \multicolumn{1}{c}{ Description } & $\begin{array}{c}\text { Thick- } \\
\text { ness } \\
\text { (feet) }\end{array}$ & $\begin{array}{c}\text { Depth } \\
\text { interval } \\
\text { (feet) }\end{array}$ \\
\hline Soil & 3 & $0-3$ \\
Clay, red and gray & 19 & $3-22$ \\
Sand, brown and white & 52 & $22-74$ \\
Clay, blue & 1 & $74-75$ \\
Sand, white & 13 & $75-88$ \\
Clay, red and blue & 45 & $88-133$ \\
Sand, white & 27 & $133-160$ \\
Clay, red & 20 & $160-180$
\end{tabular}


Well JY-65-29-705--Cont inued

$\begin{array}{lrrr}\text { Sand } & 2 & 180- & 182 \\ \text { Clay, red } & 260 & 182- & 442 \\ \text { Sand, white } & 8 & 442- & 450 \\ \text { Clay, blue } & 8 & 450- & 458 \\ \text { Sand, white } & 10 & 458-468 \\ \text { Clay, red } & 120 & 468-588\end{array}$

Well JY-65-29-706

Owner: Fort Bend County Municipal Utility District 23, Well No. 1

Driller: Layne-Texas Co.

\begin{tabular}{|c|c|c|}
\hline Description & $\begin{array}{l}\text { Thick- } \\
\text { ness } \\
\text { (feet) }\end{array}$ & $\begin{array}{l}\text { Depth } \\
\text { interval } \\
\text { (feet) }\end{array}$ \\
\hline Clay, black & 17 & $0-$ \\
\hline Sand & 71 & $17-$ \\
\hline Clay & 20 & 88- \\
\hline $\mathrm{Clay}$ and sand streaks & 112 & $108-$ \\
\hline Clay & 52 & 220 \\
\hline Sand and clay streaks & 24 & 272- \\
\hline Clay & 34 & 296- \\
\hline Sand & 68 & $330-$ \\
\hline Clay & 72 & $398-470$ \\
\hline Sand & 25 & $470-495$ \\
\hline Shale and sand streaks & 28 & $495-523$ \\
\hline Sand and shale layers & 112 & $523-$ \\
\hline Sand & 40 & $635-675$ \\
\hline Sand and shale & 36 & $675-711$ \\
\hline Sand and shale layers & 117 & $711-828$ \\
\hline Shale & 18 & 828- 846 \\
\hline Sand and shale layers & 30 & $846-876$ \\
\hline Sand & 72 & $876-948$ \\
\hline Shale & 36 & $948-984$ \\
\hline Sand & 10 & $984-994$ \\
\hline Shale & 5 & $994-999$ \\
\hline Sand & 26 & $999-1,025$ \\
\hline Shale & 17 & $1,025-1,042$ \\
\hline Sand & 70 & $1,042-1,112$ \\
\hline shale & 4 & $1,112-1,116$ \\
\hline Sand & 20 & $1,116-1,136$ \\
\hline
\end{tabular}

Well JY-65-29-706--Cont inued

$\begin{array}{lcc}\text { Sand and shale layers } & 49 & 1,136-1,185 \\ \text { Shale } & 12 & 1,185-1,197 \\ \text { Sand layers, shale and lime } & 89 & 1,197-1,286 \\ \text { Shale } & 8 & 1,286-1,294 \\ \text { Sand and shale broken } & 38 & 1,294-1,332 \\ \text { Shale } & 6 & 1,332-1,338\end{array}$

Well JY-65-29-812

Owner: Bud Romine

Driller: Almeda Hater Hell Service

\begin{tabular}{|c|c|c|c|}
\hline Description & $\begin{array}{l}\text { Thick- } \\
\text { ness } \\
\text { (feet) }\end{array}$ & $\begin{array}{l}\text { Dept } \\
\text { inter } \\
\text { (fee }\end{array}$ & $\begin{array}{l}\text { th } \\
\text { ival } \\
\text { t) }\end{array}$ \\
\hline Fill and soil & 5 & $0-$ & 5 \\
\hline Clay, red-to-gray & 14 & 5- & 19 \\
\hline Sand, brown & 10 & 19- & 29 \\
\hline clay, blue & 32 & 29- & 61 \\
\hline Sand, white & 10 & $61-$ & 71 \\
\hline clay, red & 66 & $71-$ & 137 \\
\hline Sand, brown & 14 & $137-$ & 151 \\
\hline Clay & 2 & $151-$ & 153 \\
\hline Sand, white & 19 & $153-$ & 172 \\
\hline clay, red & 1 & $172-$ & 173 \\
\hline $\begin{array}{l}\text { Hell JY- } 65-29-813 \\
\text { Owner: Fresno Fire Department } \\
\text { Driller: Almeda Water Well Servic }\end{array}$ & & & \\
\hline Description & $\begin{array}{l}\text { Thick- } \\
\text { ness } \\
\text { (feet) }\end{array}$ & $\begin{array}{r}\text { Dept } \\
\text { inter } \\
\text { (fee }\end{array}$ & $\begin{array}{l}\text { th } \\
\text { rval } \\
\text { et) }\end{array}$ \\
\hline Soil & 2 & $0-$ & 2 \\
\hline clay, white & 12 & $2-$ & 14 \\
\hline Sand, $\tan$ & 7 & $14-$ & 21 \\
\hline clay, yellow & 2 & $21-$ & 23 \\
\hline Sand, $\tan$ & 31 & $23-$ & 54 \\
\hline clay & 1 & $54-$ & 55 \\
\hline Sand, $\tan$ & 10 & $55-$ & 65 \\
\hline Clay & 2 & $65-$ & 67 \\
\hline Sand, salt and pepper with gravel & 7 & 67 & 74 \\
\hline Shale & 3 & 74- & 77 \\
\hline
\end{tabular}


Hell JY-65-33-110

Owner: Kramer Brothers

Driller: Leonard $H$. Mickelson

\begin{tabular}{|c|c|c|c|}
\hline Description & $\begin{array}{l}\text { Thick- } \\
\text { ness } \\
\text { (feet) }\end{array}$ & $\begin{array}{l}\text { Dep } \\
\text { intel } \\
\text { (fee }\end{array}$ & $\begin{array}{l}\text { th } \\
\text { rval } \\
\text { et) }\end{array}$ \\
\hline Topsoil and clay & 20 & $0-$ & 20 \\
\hline Sand & 41 & $20-$ & 61 \\
\hline Clay & 11 & $61-$ & 72 \\
\hline Sand and gravel & 83 & $72-$ & 155 \\
\hline Clay & 16 & $155-$ & 171 \\
\hline Sand, rocky & 37 & $171-$ & 208 \\
\hline Clay & 6 & 208- & 214 \\
\hline Sand & 20 & 214- & 234 \\
\hline Clay & 10 & $234-$ & 244 \\
\hline Sand, rocky & 132 & $244-$ & 376 \\
\hline Clay & 11 & $376-$ & 387 \\
\hline Sand, rocky & 83 & $387-$ & 470 \\
\hline Clay & 10 & $470-$ & 480 \\
\hline Sand, rocky & 36 & $480-$ & 516 \\
\hline Clay & 6 & $516-$ & 522 \\
\hline Sand, rocky & 52 & $522-$ & 574 \\
\hline
\end{tabular}

\begin{tabular}{lrr}
\hline \multicolumn{1}{c}{ Description } & $\begin{array}{c}\text { Thick- } \\
\text { ness } \\
\text { (feet) }\end{array}$ & $\begin{array}{c}\text { Depth } \\
\text { interval } \\
\text { (feet) }\end{array}$ \\
\hline Topsoil & 10 & $0-10$ \\
Sand & 160 & $10-170$ \\
Sand, fine gravel and logs & 109 & $170-279$ \\
Gravel and sand & 201 & $279-480$ \\
Gravel, sand and clay streaks & 273 & $480-753$ \\
Clay & 12 & $753-765$ \\
Sand & 20 & $765-785$ \\
Clay and sand streaks & 105 & $785-890$ \\
Sand and fine gravel & 38 & $890-928$ \\
Clay & 6 & $928-934$ \\
Sand & 10 & $934-944$ \\
Clay and sand streaks & 20 & $944-964$ \\
Shale & 93 & $964-1,057$ \\
Shale and sand streaks & 43 & $1,057-1,100$ \\
& &
\end{tabular}

Hell JY-65-33-209--Cont inued

Shale

$30 \quad 1,100-1,130$

Shale and sand streaks

$84 \quad 1,130-1,214$

Hell JY-65-33-304

Owner: City of Beasley

Driller: Texas Water Hells, Inc.

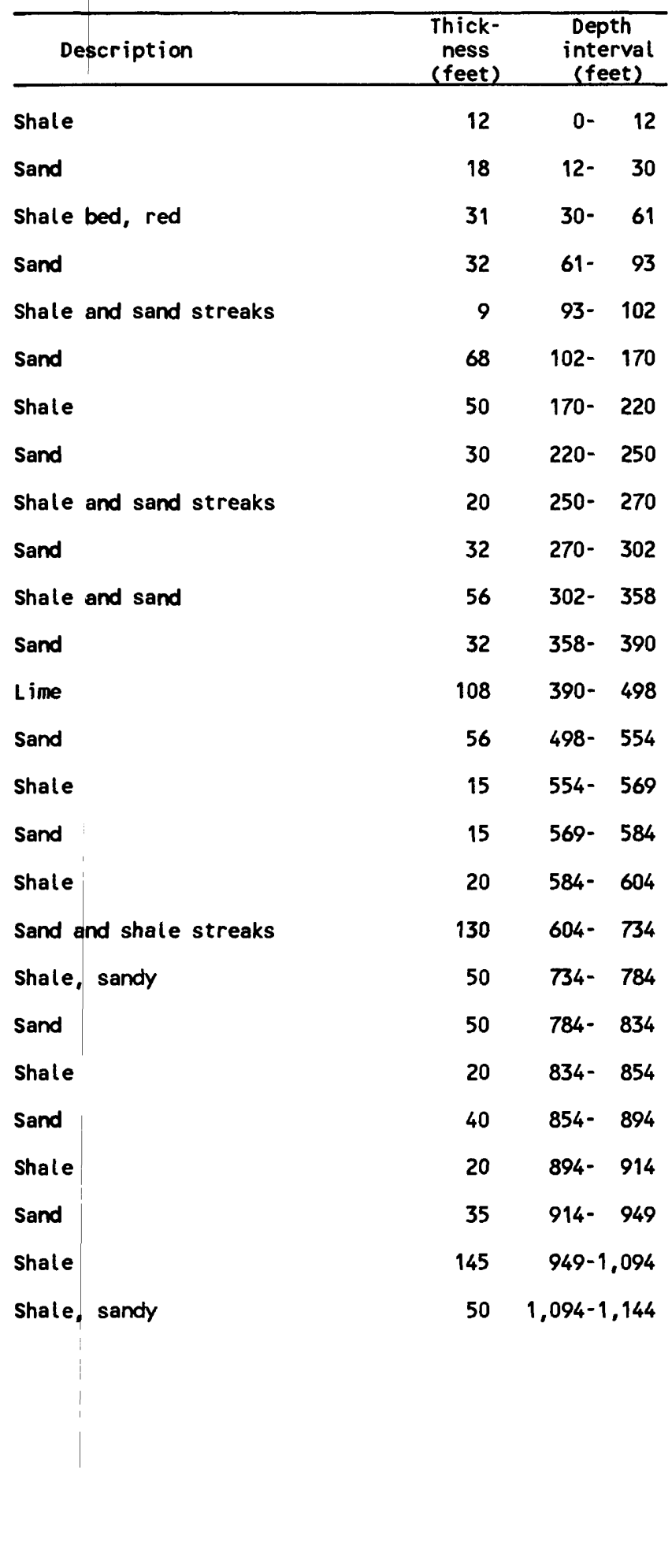


Well JY-65-33-405

Owner: Kindleton-Prude Water Supply

Driller: Katy Drilling, lnc.

\begin{tabular}{|c|c|c|}
\hline Description & $\begin{array}{l}\text { Thick- } \\
\text { ness } \\
\text { (feet) }\end{array}$ & $\begin{array}{c}\text { Depth } \\
\text { interval } \\
\text { (feet) }\end{array}$ \\
\hline $\mathrm{Clay}$ & 40 & $0-40$ \\
\hline Sand and gravel & 50 & $40-90$ \\
\hline Clay & 50 & $90-140$ \\
\hline Sand and gravel & 45 & $140-185$ \\
\hline Clay & 5 & $185-190$ \\
\hline Sand and gravel & 48 & $190-238$ \\
\hline Clay & 18 & $238-256$ \\
\hline Sand and gravel & 34 & $256-290$ \\
\hline Clay & 10 & $290-300$ \\
\hline Sand and gravel & 30 & $300-330$ \\
\hline Clay & 10 & $330-340$ \\
\hline Sand, streaks and clay & 30 & $340-370$ \\
\hline Sand streaks and clay & 45 & $370-415$ \\
\hline Shale, sandy & 35 & $415-450$ \\
\hline Sand, clay streaks & 40 & $450-490$ \\
\hline Shale & 25 & $490-515$ \\
\hline Sand & 50 & $515-565$ \\
\hline
\end{tabular}

Well JY-65-33-509

Owner: Jack Hendt

Driller: Katy Drilling, lnc.

\begin{tabular}{lccll}
\hline \multicolumn{1}{c}{ Description } & $\begin{array}{c}\text { Thick- } \\
\text { ness } \\
\text { (feet) }\end{array}$ & $\begin{array}{c}\text { Depth } \\
\text { interval } \\
\text { (feet) }\end{array}$ & & $\begin{array}{l}\text { Clay } \\
\text { Sand }\end{array}$ \\
\hline Clay, surface & 20 & $0-20$ & Clay \\
Sand & 28 & $20-48$ & Sand \\
Clay & 18 & $48-66$ & Clay \\
Sand and gravel & 85 & $66-151$ & Sand \\
Gravel & 50 & $151-201$ & Clay \\
Clay & 13 & $201-214$ & Sand and rock \\
Sand & 31 & $214-245$ & Clay \\
Clay & 27 & $245-272$ & Sand \\
Sand and rock & 36 & $272-308$ & Clay \\
Clay & 22 & $308-330$ & Sand and rock \\
Sand & 40 & $330-370$ & Clay \\
Clay & 25 & $370-395$ & \\
Sand and rock & 75 & $395-470$ &
\end{tabular}

Well JY-65-33-509--Continued

Sand $\quad 29$

Clay

Sand and rock

Clay

Sand and rock

Well JY-65-33-510

Owner: Jack Wendt

Driller: Katy Drilling, Inc.

$8 \quad 470-478$

478-507

$507-517$

517-550

550-564

564-623

\begin{tabular}{|c|c|c|}
\hline Description & $\begin{array}{c}\text { Thick- } \\
\text { ness } \\
\text { (feet) }\end{array}$ & $\begin{array}{l}\text { Depth } \\
\text { interval } \\
\text { (feet) }\end{array}$ \\
\hline Clay, surface & 14 & $0-14$ \\
\hline Sand & 90 & $14-104$ \\
\hline Clay & 66 & $104-170$ \\
\hline Sand and gravel & 33 & $170-203$ \\
\hline Clay & 9 & $203-212$ \\
\hline Sand and gravel & 44 & $212-256$ \\
\hline Clay & 9 & $256-265$ \\
\hline Sand and gravel & 42 & $265-307$ \\
\hline Clay & 18 & $307-325$ \\
\hline Sand & 27 & $325-352$ \\
\hline Clay & 18 & $352-370$ \\
\hline Sand & 16 & $370-386$ \\
\hline Clay & 7 & $386-393$ \\
\hline Sand & 19 & $393-412$ \\
\hline Clay & 6 & $412-418$ \\
\hline Sand & 33 & $418-451$ \\
\hline Clay & 20 & $451-471$ \\
\hline Sand & 24 & $471-495$ \\
\hline Clay & 13 & $495-508$ \\
\hline Sand and rock & 24 & $508-532$ \\
\hline Clay & 7 & $532-539$ \\
\hline Sand & 6 & $539-545$ \\
\hline Clay & 5 & $545-550$ \\
\hline Sand and rock & 41 & $550-591$ \\
\hline $\mathrm{Clay}$ & 9 & $591-600$ \\
\hline
\end{tabular}


Well JY-65-33-609

Owner: Clarence Danklefs

Driller: Katy Drilling, Inc.

\begin{tabular}{|c|c|c|}
\hline Description & $\begin{array}{l}\text { Thick- } \\
\text { ness } \\
\text { (feet) }\end{array}$ & $\begin{array}{c}\text { Depth } \\
\text { interval } \\
\text { (feet) }\end{array}$ \\
\hline Topsoil and clay & 10 & $0-10$ \\
\hline Sand & 30 & $10-40$ \\
\hline Sand and $\mathrm{clay}$ breaks & 72 & $40-112$ \\
\hline Clay & 22 & $112-134$ \\
\hline Sand & 18 & $134-152$ \\
\hline Clay & 8 & $152-160$ \\
\hline Sand & 17 & $160-177$ \\
\hline $\mathrm{Clay}$ & 8 & $177-185$ \\
\hline Sand and small clay breaks & 71 & $185-256$ \\
\hline Clay & 5 & $256-261$ \\
\hline Sand & 30 & $261-291$ \\
\hline Clay & 30 & $291-321$ \\
\hline Sand & 42 & $321-363$ \\
\hline Clay & 24 & $363-387$ \\
\hline Sand & 63 & $387-450$ \\
\hline Clay & 12 & $450-462$ \\
\hline Sand and rock & 132 & $462-594$ \\
\hline Clay & 25 & $594-619$ \\
\hline
\end{tabular}

Well JY-65-33-610

Owner: Alboa Grass Farm

Driller: 0. T. Davis and Sons

\begin{tabular}{lcc}
\hline \multicolumn{1}{c}{ Description } & $\begin{array}{c}\text { Thick- } \\
\text { ness } \\
\text { (feet) }\end{array}$ & $\begin{array}{c}\text { Depth } \\
\text { interval } \\
\text { (feet) }\end{array}$ \\
\hline Clay, red & 4 & $0-4$ \\
Sand, fine & 30 & $4-34$ \\
Clay & 31 & $34-65$ \\
Clay & 19 & $65-84$ \\
Sand and gravel & 36 & $84-120$ \\
Clay and shale & 38 & $120-158$ \\
Gravel & 22 & $158-180$ \\
Sand, hard broken & 3 & $180-183$ \\
Sand and gravel & 38 & $183-221$ \\
Shale & 8 & $221-229$ \\
Sand and gravel & 18 & $229-247$
\end{tabular}

Well JY-65-33-611

Owner: T. W. Sod, Limited

Driller: Critendon Drilling Service, Inc.

\begin{tabular}{lrr}
\hline \multicolumn{1}{c}{ Description } & $\begin{array}{c}\text { Thick- } \\
\text { ness } \\
\text { (feet) }\end{array}$ & $\begin{array}{c}\text { Depth } \\
\text { interval } \\
\text { (feet) }\end{array}$ \\
\hline Sand, surface & 4 & $0-4$ \\
Clay, brown & 60 & $4-64$ \\
Sand, fine, brown & 9 & $64-73$ \\
Clay, brown & 36 & $73-109$ \\
Sand and gravel, brown & 29 & $109-138$ \\
Sand, white, medium-to-coarse & 26 & $138-164$ \\
Clay and sand & 3 & $164-167$ \\
Sand & 2 & $167-169$ \\
Clay, brown & 16 & $169-185$
\end{tabular}

Well JY-65-33-612

Owner: T. H. Sod, Limited

Driller: Critendon Drilling Service, Inc.

\begin{tabular}{lcc}
\hline \multicolumn{1}{c}{ Description } & $\begin{array}{c}\text { Thick- } \\
\text { ness } \\
\text { (feet) }\end{array}$ & $\begin{array}{c}\text { Depth } \\
\text { interval } \\
\text { (feet) }\end{array}$ \\
\hline Sand, surface & 4 & $0-4$ \\
Clay, brown & 60 & $4-64$ \\
Sand, fine, brown & 9 & $64-73$ \\
Clay, brown & 36 & $73-109$ \\
Sand and gravel & 29 & $109-138$ \\
Sand, white, medium-to-coarse & 26 & $138-164$ \\
Clay and sand & 3 & $164-167$ \\
Sand & 2 & $167-169$ \\
Clay, brown & 16 & $169-185$ \\
Well JY-65-33-906 & & \\
Owner: Alboa Grass Farm & & \\
Driller: 0. T. Davis and Sons & & $60-80$ \\
\hline
\end{tabular}


Well JY-65-33-906--Cont inued

$\begin{array}{lrr}\text { Gravel } & 60 & 120-180 \\ \text { Clay } & 5 & 180-185 \\ \text { Gravel } & 25 & 185-210 \\ \text { Clay } & 20 & 210-230 \\ \text { Gravel } & 25 & 230-255 \\ \text { Clay } & 5 & 255-260 \\ \text { Sand and gravel } & 5 & 260-285\end{array}$

Well JY-65-33-907

Owner: D. F. G. Farms

Driller: Pesak Water Well Service

\begin{tabular}{lcc}
\hline \multicolumn{1}{c}{ Description } & $\begin{array}{c}\text { Thick- } \\
\text { ness } \\
\text { (feet) }\end{array}$ & $\begin{array}{c}\text { Depth } \\
\text { interval } \\
\text { (feet) }\end{array}$ \\
\hline Clay & 15 & $0-15$ \\
Sand & 6 & $15-21$ \\
Clay & 31 & $21-52$ \\
Sand & 53 & $52-105$ \\
Clay & 15 & $105-120$ \\
Sand & 10 & $120-130$ \\
Sand, coarse & 45 & $130-175$ \\
Gravel & 20 & $175-195$ \\
& & $13-40$ \\
Well JY-65-34-714 & & \\
Owner: Tom Bosse \\
Driller: Critendon Drilling Service, inc.
\end{tabular}

Well JY-65-35-306

Owner: Houston Lighting and Power Co., Well No. 4

Driller: Layne-Texas Co.

\begin{tabular}{|c|c|c|}
\hline Description & $\begin{array}{l}\text { Thick- } \\
\text { ness } \\
\text { (feet) }\end{array}$ & $\begin{array}{c}\text { Depth } \\
\text { interval } \\
\text { (feet) }\end{array}$ \\
\hline Clay & 17 & $0-17$ \\
\hline Sand & 16 & $17-33$ \\
\hline Clay & 29 & $33-62$ \\
\hline Sand & 21 & $62-83$ \\
\hline Clay & 9 & $83-92$ \\
\hline Sand & 27 & $92-119$ \\
\hline Clay & 68 & $119-187$ \\
\hline Sand & 25 & $187-212$ \\
\hline Shale, sandy and sand & 25 & $212-237$ \\
\hline Shale and sandy shale & 47 & $237-284$ \\
\hline Sand and shale streaks & 14 & $284-298$ \\
\hline Shale & 21 & $298-319$ \\
\hline Sand and sandy shale & 7 & $319-326$ \\
\hline Shale and sand streaks & 45 & $326-371$ \\
\hline Sand and sandy shale & 32 & $371-403$ \\
\hline Shale & 17 & $403-420$ \\
\hline Shale, sandy & 9 & $420-429$ \\
\hline Shale & 5 & $429-434$ \\
\hline Sand and shale streaks & 78 & $434-512$ \\
\hline Shale & 7 & $512-519$ \\
\hline Sand and shale & 29 & $519-548$ \\
\hline Sand & 10 & $548-558$ \\
\hline Sand and shale streaks & 10 & $558-568$ \\
\hline Shale & 12 & $568-580$ \\
\hline Sand and sandy shale & 30 & $580-610$ \\
\hline Shale & 6 & $610-616$ \\
\hline Sand and shale breaks & 15 & $616-631$ \\
\hline Shale & 26 & $631-657$ \\
\hline Sand and shale breaks & 31 & $657-688$ \\
\hline Shale and sand streaks & 14 & $688-702$ \\
\hline Sand and shale streaks & 26 & $702-728$ \\
\hline Sand and shale & 18 & $728-746$ \\
\hline Sand & 15 & $746-761$ \\
\hline Shale & 4 & $761-765$ \\
\hline
\end{tabular}


Well JY-65-35-306--Cont inued

Sand and shale
Shale
Sand and sandy shale
Shale and sand streaks
Sand and shale
Shale
Well JY-65-35-307
Owner: Houston Lighting and Power Co.,
Driller: Layne-Texas Co.

\begin{tabular}{|c|c|c|c|c|c|}
\hline Description & $\begin{array}{l}\text { Thick- } \\
\text { ness } \\
\text { (feet) }\end{array}$ & $\begin{array}{l}\text { Depth } \\
\text { interval } \\
\text { (feet) }\end{array}$ & $\begin{array}{l}\text { Well JY- } 65-35-308 \\
\text { Owner: Houston Lighting and Power }\end{array}$ & Co. , & \\
\hline Fill & 2 & $0-2$ & Driller: Alsay-Texas Corporation & & \\
\hline $\begin{array}{l}\text { Clay } \\
\text { Sand and gravel }\end{array}$ & $\begin{array}{r}6 \\
114\end{array}$ & $\begin{array}{l}2-8 \\
8-122\end{array}$ & Description & $\begin{array}{l}\text { Thick- } \\
\text { ness } \\
\text { (feet) }\end{array}$ & $\begin{array}{c}\text { Depth } \\
\text { interval } \\
\text { (feet) }\end{array}$ \\
\hline Shale & 10 & $122-132$ & Clay, gray & 20 & $0-20$ \\
\hline Sand & 15 & $132-147$ & Sand & 5 & $20-25$ \\
\hline Shale & 53 & $147-200$ & clay, red & 50 & $25-75$ \\
\hline Sand & 28 & $200-228$ & Shale & 5 & $75-80$ \\
\hline Shale and sand streaks & 54 & $228-282$ & Sand & 35 & $80-115$ \\
\hline Sand & 19 & $282-301$ & Shale & 9 & $115-124$ \\
\hline Shale & 19 & $301-320$ & Clay & 6 & $124-130$ \\
\hline Sand & 19 & $320-339$ & Sand & 35 & $130-165$ \\
\hline Shale & 36 & $339-375$ & Shale and blue clay & 52 & $165-217$ \\
\hline Sand & 25 & $375-400$ & Sand and small gravel & 25 & $217-242$ \\
\hline Sand, gravel and shale streaks & 37 & $400-437$ & $\mathrm{Clay}$ and sand & 8 & $242-250$ \\
\hline Sand & 63 & $437-500$ & clay, blue and sand & 24 & $250-274$ \\
\hline Shale & 9 & $500-509$ & Clay and gravel & 31 & $274-305$ \\
\hline Sand, broken with shale & 21 & $509-530$ & Shale and gravel & 32 & $305-337$ \\
\hline Shale & 6 & $530-536$ & clay, blue & 60 & $337-397$ \\
\hline Sand & 29 & $536-565$ & Gravel & 3 & $397-400$ \\
\hline Shale & 17 & $565-582$ & clay, blue & 92 & $400-492$ \\
\hline Sand, broken with shale & 17 & $582-599$ & Sand and gravel & 127 & $492-619$ \\
\hline Shale & 19 & $599-618$ & clay, blue & 12 & $619-631$ \\
\hline Sand & 20 & $618-638$ & Sand & 9 & $631-640$ \\
\hline Shale & 11 & $638-649$ & Clay & 9 & $640-649$ \\
\hline Sand & 10 & $649-659$ & Sand & 58 & $649-707$ \\
\hline Shale & 9 & $659-668$ & Clay & 4 & $707-711$ \\
\hline
\end{tabular}

Well JY-65-35-307-- Cont inued

$\begin{array}{rl}20 & 765-785 \\ 6 & 785-791 \\ 47 & 791-838 \\ 5 & 838-843 \\ 6 & 843-849 \\ 2 & 849-851\end{array}$

Sand 26

$668-694$

Shale 16

$694-710$

Sand

21

710-731

Shale

26

$731-757$

Sand

18

757-775

Shale

$775-790$

Sand

$790-802$

Sand, broken with shale

$802-840$

Sand, broken with shale

38

$802-840$

Shale

10

$840-850$

Driller: Layne-Texas co. 
Hell JY-65-35-308--Continued

$\begin{array}{rrr}\text { Sand } & 133 & 711-844 \\ \text { Clay } & 6 & 844-850\end{array}$

Well JY-65-35-716

Owner: R. B. Hobbs

Driller: Katy Drilling, Inc.

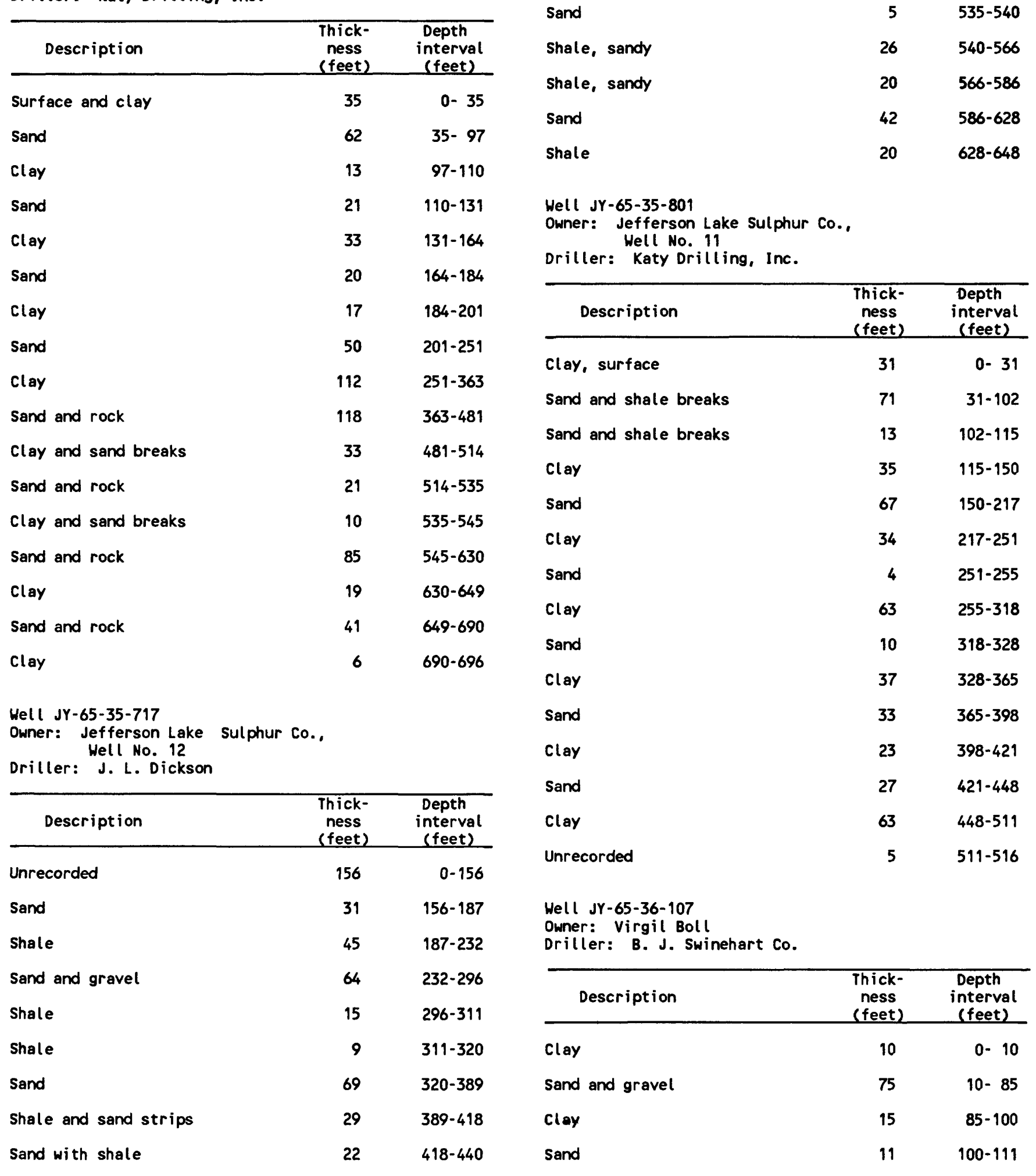

Hell JY-65-35-717--Cont inued

$\begin{array}{lcc}\text { Sand and gravel } & 46 & 440-486 \\ \text { Shale } & 7 & 486-493 \\ \text { Sand and gravel } & 21 & 493-514 \\ \text { Shale, sticky } & 21 & 514-535 \\ \text { Sand } & 5 & 535-540 \\ \text { Shale, sandy } & 26 & 540-566 \\ \text { Shale, sandy } & 20 & 566-586 \\ \text { Sand } & 42 & 586-628 \\ \text { Shale } & 20 & 628-648\end{array}$

Well JY-65-35-801 
Table 3.--Drillers' logs of selected wells in Fort Bend County, 1969-87--Cont inued

Well JY-65-36-107--Cont inued

\begin{tabular}{|c|c|c|}
\hline clay & 31 & $111-142$ \\
\hline Sand & 28 & $142-170$ \\
\hline Clay & 23 & $170-193$ \\
\hline Sand & 15 & $193-208$ \\
\hline Clay & 15 & $208-223$ \\
\hline Sand & 15 & $223-238$ \\
\hline \multicolumn{3}{|c|}{$\begin{array}{l}\text { Well JY-65-36-209 } \\
\text { Owner: Champl in Petroleum } \\
\text { Driller: 0'Day Drlg Co., Inc. }\end{array}$} \\
\hline Description & $\begin{array}{c}\text { Thick- } \\
\text { ness } \\
\text { (feet) }\end{array}$ & $\begin{array}{c}\text { Depth } \\
\text { interval } \\
\text { (feet) }\end{array}$ \\
\hline Topsoil & 4 & $0-4$ \\
\hline Clay & 14 & $4-18$ \\
\hline Sand & 22 & $18-40$ \\
\hline clay & 90 & $40-130$ \\
\hline Sand & 24 & $130-154$ \\
\hline Clay & 62 & $154-216$ \\
\hline Sand & 13 & $216-229$ \\
\hline Clay & 66 & $229-295$ \\
\hline Sand & 52 & $295-347$ \\
\hline
\end{tabular}

Well JY-65-36-510

Owner: Exxon Company, U.S.A. Thompson's field office, Well No. 1

Driller: H. B. Patterson

\begin{tabular}{lcc}
\hline \multicolumn{1}{c}{ Description } & $\begin{array}{c}\text { Thick- } \\
\text { ness } \\
\text { (feet) }\end{array}$ & $\begin{array}{c}\text { Depth } \\
\text { interval } \\
\text { (feet) }\end{array}$ \\
\hline Soil & 10 & $0-10$ \\
Sand & 10 & $10-20$ \\
Shale & 29 & $29-49$ \\
Sand & 66 & $49-115$ \\
Shale & 7 & $115-122$ \\
Sand & 7 & $122-129$ \\
Shale & 21 & $129-150$ \\
Sand & 10 & $150-160$ \\
Shale & 15 & $160-175$ \\
Sand & 14 & $175-189$ \\
Shale & 34 & $189-223$ \\
Sand & 22 & $223-245$
\end{tabular}

Well JY-65-36-510--Cont inued

$\begin{array}{lrr}\text { Shale } & 2 & 245-247 \\ \text { Sand } & 3 & 247-250 \\ \text { Shale } & 114 & 250-364 \\ \text { Sand } & 25 & 364-389 \\ \text { Shale } & 51 & 389-440 \\ \text { Sand and rock } & 1 & 440-441 \\ \text { Sand } & 54 & 441-495\end{array}$

Well JY-65-37-201

Owner: Cont inental Homes Co.

Driller: Almeda Water Well Service

\begin{tabular}{lcr}
\hline \multicolumn{1}{c}{ Description } & $\begin{array}{c}\text { Thick- } \\
\text { ness } \\
\text { (feet) }\end{array}$ & $\begin{array}{c}\text { Depth } \\
\text { interval } \\
\text { (feet) }\end{array}$ \\
\hline Soil & 2 & $0-2$ \\
Clay, gray-to-red & 12 & $2-14$ \\
Sand, brown & 25 & $14-39$ \\
Clay, blue & 2 & $39-41$ \\
Sand, white & 26 & $41-67$ \\
Clay & 1 & $67-68$
\end{tabular}

Well JY-65-37-202

Owner: R. L. Cooper

Driller: Abner J. Joebl in

\begin{tabular}{|c|c|c|}
\hline Description & $\begin{array}{c}\text { Thick- } \\
\text { ness } \\
\text { (feet) }\end{array}$ & $\begin{array}{c}\text { Depth } \\
\text { interval } \\
\text { (feet) }\end{array}$ \\
\hline Soil, black & 6 & $0-6$ \\
\hline clay, red & 24 & 6- 30 \\
\hline Unrecorded & 10 & $30-40$ \\
\hline \multicolumn{3}{|c|}{$\begin{array}{l}\text { Well JY-65-41-305 } \\
\text { Owner: Anton Rychlik } \\
\text { Driller: Crowell Drilling Co. }\end{array}$} \\
\hline Description & $\begin{array}{l}\text { Thick- } \\
\text { ness } \\
\text { (feet) }\end{array}$ & $\begin{array}{c}\text { Depth } \\
\text { interval } \\
\text { (feet) }\end{array}$ \\
\hline Clay & 7 & $0-7$ \\
\hline Sand & 23 & $7-30$ \\
\hline Clay & 10 & $30-40$ \\
\hline Sand & 80 & $40-120$ \\
\hline Shale, red sandy & 40 & $120-160$ \\
\hline Shale & 20 & $160-180$ \\
\hline Sand & 10 & $180-190$ \\
\hline Shale & 3 & $190-193$ \\
\hline
\end{tabular}


Well JY-65-41-305--Cont inued

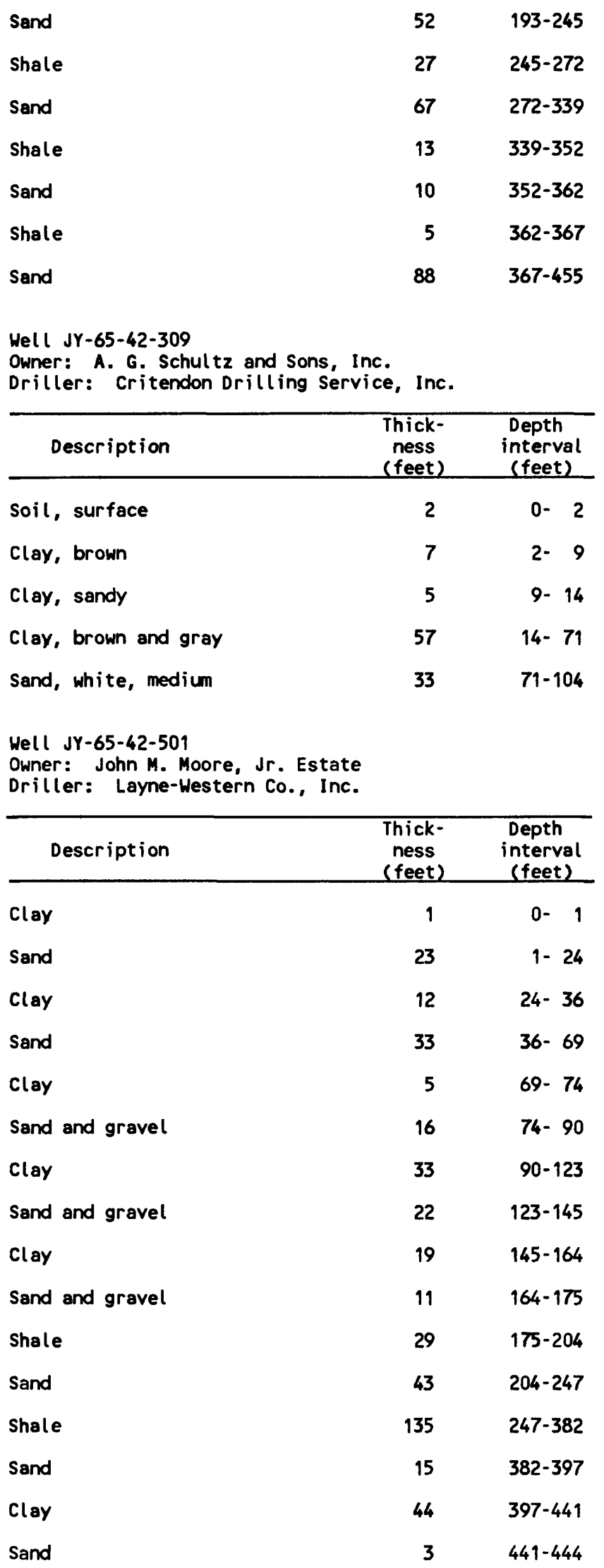

Well JY-65-42-501--Cont inued

\begin{tabular}{|c|c|c|}
\hline clay & 36 & $444-480$ \\
\hline Sand & 21 & $480-501$ \\
\hline clay & 50 & $501-551$ \\
\hline Sand & 24 & $551-575$ \\
\hline Clay & 24 & $575-599$ \\
\hline Sand & 13 & $599-612$ \\
\hline Clay & 58 & $612-670$ \\
\hline Sand & 14 & $670-684$ \\
\hline clay & 44 & $684-728$ \\
\hline Sand & 6 & $728-734$ \\
\hline Clay & 5 & $734-739$ \\
\hline Sand & 91 & $739-830$ \\
\hline Clay & 41 & $830-87$ \\
\hline Sand & 8 & $871-879$ \\
\hline Clay & 4 & $879-883$ \\
\hline Sand & 3 & $883-886$ \\
\hline Clay & 37 & $886-923$ \\
\hline Sand & 3 & $923-926$ \\
\hline Clay & 13 & $926-939$ \\
\hline
\end{tabular}

Well JY-66-24-302

Owner: Jim Skipton

Driller: Bussell and Son, Inc.

\begin{tabular}{lcr}
\hline Description & $\begin{array}{c}\text { Thick- } \\
\text { ness } \\
\text { (feet) }\end{array}$ & $\begin{array}{c}\text { Depth } \\
\text { interval } \\
\text { (feet) }\end{array}$ \\
\hline Clay, red & 3 & $0-3$ \\
Gravel & 33 & $3-36$ \\
Clay & 80 & $36-116$ \\
Sand & 40 & $116-156$
\end{tabular}

Well JY-66-32-912

Owner: Tilford Sulak

Driller: Richter Water Well Drilling

\begin{tabular}{lcr}
\hline \multicolumn{1}{c}{ Description } & $\begin{array}{c}\text { Thick- } \\
\text { ness } \\
\text { (feet) }\end{array}$ & $\begin{array}{c}\text { Depth } \\
\text { interval } \\
\text { (feet) }\end{array}$ \\
\hline Topsoil & 1 & $0-1$ \\
Clay, blue and white & 4 & $1-5$ \\
Clay, pink & 49 & $5-54$ \\
Sand and gravel & 86 & $54-140$ \\
Clay, sand and gravel & 40 & $140-180$
\end{tabular}


Table 3.--prillers' logs of selected wells in Fort Bend County, 1969-87--Continued

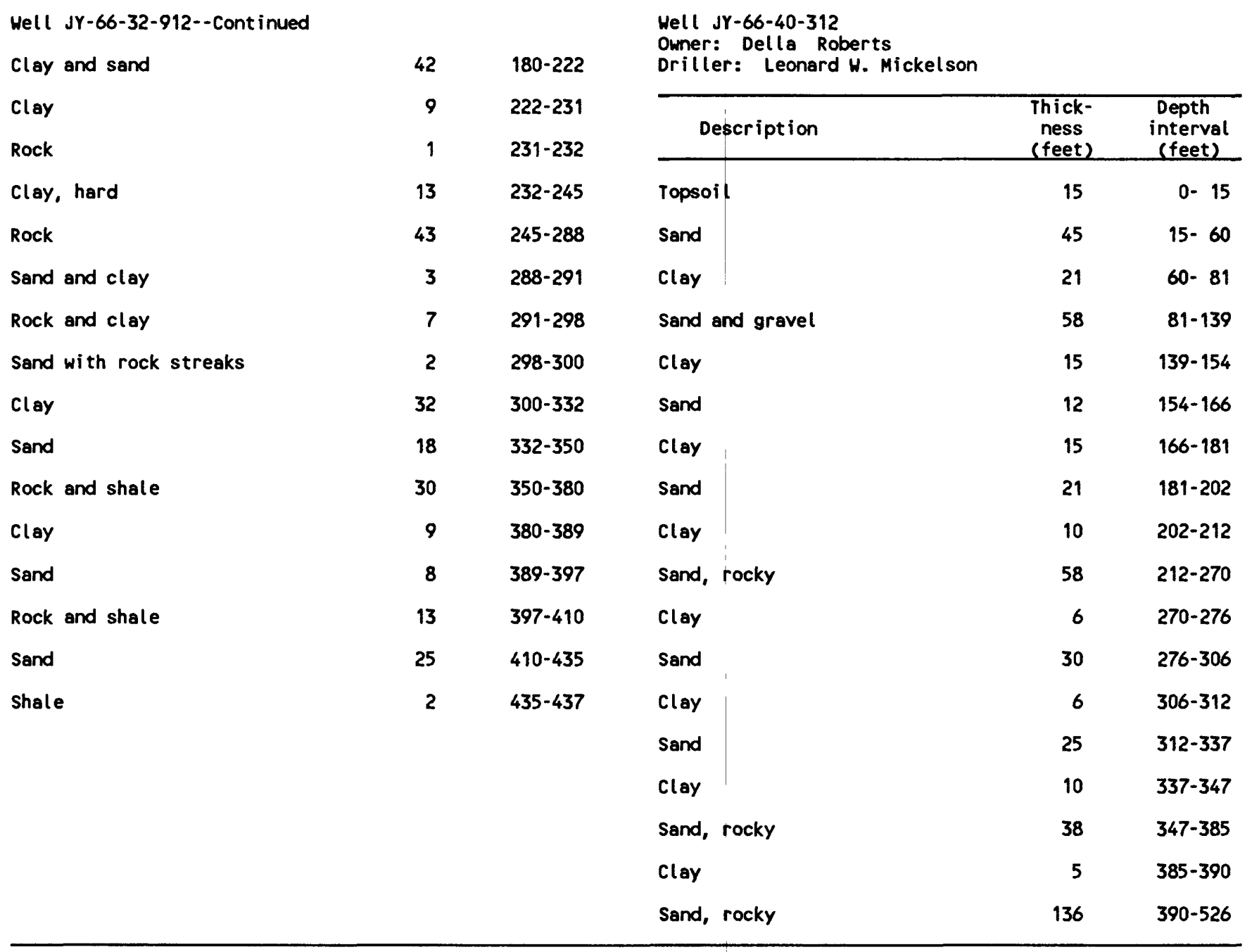


[Water levels in feet below land surface; screen, interval; altitude, altitude of land surface above sea level]

Hell JY-65-09-906

Owner: Don McMillian, Well no.?

Screen: $83-575$ feet

Altitude: 149 feet

\begin{tabular}{cc}
\hline Date & \multicolumn{1}{c}{$\begin{array}{c}\text { Water } \\
\text { level }\end{array}$} \\
\hline $03-13-69$ & 97.61 \\
$03-06-70$ & 99.70 \\
$03-11-71$ & 99.34 \\
$03-05-73$ & 105.20 \\
$03-05-74$ & 105.12 \\
$02-25-75$ & 106.72 \\
$02-27-76$ & 108.79 \\
$03-07-77$ & 108.19 \\
$11-17-77$ & 111.69 \\
$03-10-78$ & 108.68 \\
$11-17-78$ & 112.42 \\
$03-15-79$ & 116.16 \\
$12-10-79$ & 118.09 \\
$01-18-80$ & 113.93 \\
$12-15-80$ & 117.86 \\
$01-15-81$ & 115.97 \\
$10-30-81$ & 119.52 \\
$10-25-82$ & 120.66 \\
$02-06-87$ & 113.65 \\
&
\end{tabular}

Well JY-65-10-702

Owner: Earl McMillian

Screen: 176-346 feet

Altitude: 144 feet

\begin{tabular}{cc}
\hline Date & $\begin{array}{c}\text { Water } \\
\text { level }\end{array}$ \\
\hline $03-13-69$ & 101.28 \\
$03-06-70$ & 107.09 \\
$03-11-71$ & 102.71 \\
$02-28-72$ & 109.72 \\
$03-05-73$ & 109.88 \\
$03-05-74$ & 111.49 \\
$02-25-75$ & 107.82 \\
$02-27-76$ & 110.37 \\
$03-07-77$ & 109.27 \\
$11-17-77$ & 111.05 \\
$03-10-78$ & 109.61 \\
$11-17-78$ & 111.96 \\
$12-10-79$ & 113.76 \\
$01-18-80$ & 111.82 \\
$12-15-80$ & 114.04 \\
$01-15-81$ & 113.13 \\
$10-30-81$ & 114.42 \\
$02-01-82$ & 117.23 \\
$10-25-82$ & 118.78 \\
$01-17-83$ & 118.45 \\
$09-26-83$ & 119.16 \\
$01-19-84$ & 115 \\
$01-11-85$ & 120.51 \\
$01-07-86$ & 121.65 \\
$01-07-87$ & 120.02 \\
&
\end{tabular}

Well JY-65-10-703

Owner: P. V. Cook, Well no. 3

Depth: 170 feet

Altitude: 140 feet

\begin{tabular}{cc}
\hline Date & $\begin{array}{c}\text { Water } \\
\text { level }\end{array}$ \\
\hline $03-12-69$ & 97.8 \\
$03-02-70$ & 99.33 \\
$03-11-71$ & 100.68 \\
$02-23-72$ & 102.40 \\
$03-05-73$ & 103.60 \\
$03-05-74$ & 104.48 \\
$02-25-75$ & 106.22 \\
$02-27-76$ & 107.75 \\
$03-04-77$ & 107.89 \\
$11-17-77$ & 108.91 \\
$03-10-78$ & 108.22 \\
$11-17-78$ & 109.73 \\
$02-14-79$ & 109.89 \\
$12-10-79$ & 110.02 \\
$01-18-80$ & 109.82 \\
$12-15-80$ & 110.17 \\
$01-15-81$ & 110.01 \\
$10-30-81$ & 110.15 \\
$02-01-82$ & 110 \\
$11-10-82$ & 111.26 \\
$01-17-83$ & 114.35 \\
$09-26-83$ & 114.84 \\
$01-19-84$ & 114.85 \\
$01-22-85$ & 115.76 \\
&
\end{tabular}

Well JY-65-10-801

Owner: Clyde Nelson

Screen: 92-365 feet

Altitude: 133 feet

\begin{tabular}{cl}
\hline Date & \multicolumn{1}{c}{$\begin{array}{l}\text { Water } \\
\text { level }\end{array}$} \\
\hline $02-19-86$ & 112.57 \\
$02-06-87$ & 113
\end{tabular}

Well JY-65-10-812

Owner: City of Katy, Well no. 5

Screen: 444-634 feet

Alt itude: 129 feet

\begin{tabular}{ll}
\hline \multicolumn{1}{c}{ Date } & $\begin{array}{l}\text { Water } \\
\text { level }\end{array}$ \\
\hline $07--81$ & 167 \\
$03-03-83$ & 148.08 \\
$03-18-83$ & 154.49 \\
$08-08-83$ & 169.02 \\
$11-03-83$ & 173.51 \\
$01-25-84$ & 153.09 \\
$02-23-84$ & 150.88 \\
$03-29-84$ & 144.91 \\
$04-25-84$ & 169.67 \\
$06-07-84$ & 183.58 \\
$07-30-84$ & 184.87 \\
$09-27-84$ & 195.10 \\
$11-14-84$ & 171.21 \\
$11-30-84$ & 165 \\
$12-28-84$ & 156.52 \\
$01-09-85$ & 153.34 \\
$01-07-86$ & 152.02 \\
$03-11-86$ & 156.30
\end{tabular}

Well JY-65-10-812-Cont.

$\begin{array}{ll}01-07-87 & 156.74 \\ 02-06-87 & 153.60\end{array}$

Well JY-65-17-201

Owner: Richard A. Woods Screen: 100-335 feet Altitude: 157 feet

\begin{tabular}{cc}
\hline Date & $\begin{array}{c}\text { Water } \\
\text { level }\end{array}$ \\
\hline $03-14-69$ & 93.71 \\
$03-05-70$ & 95.08 \\
$03-10-71$ & 96.83 \\
$02-23-72$ & 98.08 \\
$03-05-73$ & 99.92 \\
$03-05-74$ & 99.10 \\
$03-11-75$ & 99.42 \\
$03-03-76$ & 101.29 \\
$02-28-77$ & 100.66 \\
$11-17-77$ & 102.73 \\
$03-14-78$ & 101.16 \\
$11-17-78$ & 103.09 \\
$02-26-79$ & 103.64 \\
$12-10-79$ & 104.37 \\
$01-18-80$ & 103.93 \\
$01-15-81$ & 105.54 \\
$10-30-81$ & 106.75 \\
$02-01-82$ & 105.04 \\
$11-10-82$ & 108.36 \\
$01-17-83$ & 108.10 \\
$09-22-83$ & 108.41 \\
$01-25-84$ & 108.53 \\
$01-23-85$ & 105.23 \\
$01-28-86$ & 104.65 \\
$01-08-87$ & 104.02 \\
&
\end{tabular}

Well JY-65-17-204

Owner: Richard A. Woods Depth: 330 feet

Altitude: 157 feet

\begin{tabular}{lc}
\hline Date & \multicolumn{1}{c}{$\begin{array}{c}\text { Water } \\
\text { level }\end{array}$} \\
\hline $03-14-69$ & 96.33 \\
$03-05-70$ & 97.93 \\
$03-10-71$ & 99.58 \\
$02-23-72$ & 101.04 \\
$03-05-73$ & 101.52 \\
$03-05-74$ & 101.59 \\
$03-11-75$ & 102.11 \\
$03-03-76$ & 102.90 \\
$02-28-77$ & 103.64 \\
$11-17-77$ & 106.84 \\
$03-14-78$ & 104.17 \\
$11-16-78$ & 107.72 \\
$02-26-79$ & 105.64 \\
$12-10-79$ & 107.85 \\
$01-18-80$ & 106.66 \\
$12-15-80$ & 108.02 \\
$01-15-81$ & 107.90 \\
$10-30-81$ & 110.50 \\
$02-01-82$ & 109.31 \\
$11-10-82$ & 113.51 \\
$01-17-83$ & 113.13 \\
$09-22-83$ & 113.82
\end{tabular}


Well JY-65-17-204--Cont.

$\begin{array}{ll}01-25-84 & 120.17 \\ 01-23-85 & 117.10\end{array}$

Hell JY-65-17-307

Owner: Walter P. Cook

Screen: 232-617 feet

Altitude: 147 feet

\begin{tabular}{ll}
\hline Date & $\begin{array}{l}\text { Water } \\
\text { level }\end{array}$ \\
\hline $02-14-86$ & $\begin{array}{l}114.10 \\
03-20-87\end{array}$ \\
\end{tabular}

Well JY-65-17-401

Owner: Vernon $\mathrm{W}$. Frost

Screen: $85-378$ feet

Altitude: 114 feet

\begin{tabular}{ll}
\hline Date & $\begin{array}{l}\text { Water } \\
\text { level }\end{array}$ \\
\hline $02-24-87$ & 43
\end{tabular}

02-24-87 $\quad 43$

Hell JY-65-17-402

Owner: Gail H. Spencer

Screen: 117-367 feet

Altitude: 112 feet

\begin{tabular}{ll}
\hline Date & $\begin{array}{l}\text { Water } \\
\text { level }\end{array}$ \\
\hline
\end{tabular}

$02-24-87 \quad 40.45$

Well JY-65-17-404

Owner: Southern Pacific Rail road Co.

Depth: 1,100 feet

Altitude: 114 feet

\begin{tabular}{ll}
\hline Date & $\begin{array}{l}\text { Water } \\
\text { level }\end{array}$ \\
\hline
\end{tabular}

01-29-70 60.32

08-13-70 $\quad 74.5$

$01-18-72 \quad 61.68$

08-08-72 $\quad 69.15$

$01-23-73 \quad 55.90$

$08-14-73 \quad 71$

01-16-74 $\quad 62.6$

08-19-74 75.81

$01-16-75 \quad 64.39$

$08-12-75 \quad 69.83$

$01-19-76 \quad 68.39$

08-04-76 $\quad 76.05$

$01-12-77 \quad 67.79$

08-02-77 77.41

03-01-79 $\quad 71.35$

$01-15-80 \quad 73.33$

08-06-80 72.29

$01-26-81 \quad 72.71$

08-11-81 73.01

03-01-82 72.52

07-19-82 $\quad 72.45$

$02-02-83 \quad 73.92$

08-10-83 $\quad 82.76$

$01-27-84 \quad 72.26$

08-02-84 $\quad 84.71$

$01-21-85 \quad 79.19$

09-18-85 $\quad 88.17$

02-03-86 $\quad 83.56$

02-24-87 77.15
Hell JY-65-18-101

Owner: C. C. Cardiff

Depth: 818 feet

Altitude: 142 feet

\begin{tabular}{lc}
\hline Date & $\begin{array}{c}\text { Mater } \\
\text { level }\end{array}$ \\
\hline $03-12-69$ & 97.47 \\
$03-02-70$ & 100.89 \\
$03-11-71$ & 101.08 \\
$02-23-72$ & 105.92 \\
$03-05-73$ & 104.30 \\
$03-05-74$ & 104.78 \\
$02-25-75$ & 106.22 \\
$02-27-76$ & 108.72 \\
$03-07-77$ & 108.72 \\
$11-17-77$ & 111.23 \\
$03-14-78$ & 107.60 \\
$11-16-78$ & 112.37 \\
$02-08-79$ & 112.60 \\
$12-10-79$ & 112.98 \\
$01-18-80$ & 112.91 \\
$12-15-80$ & 113.21 \\
$01-15-81$ & 113.02 \\
$10-29-81$ & 114.20 \\
$02-01-82$ & 112.93 \\
$11-10-82$ & 115.43 \\
$01-17-83$ & 116.03 \\
$09-22-83$ & 117.87 \\
$01-25-84$ & 119.60 \\
$01-22-85$ & 118.50 \\
$01-07-87$ & 118.12 \\
$02-25-87$ & 114.62 \\
&
\end{tabular}

Hell JY-65-18-103

Owner: C. C. Cardiff, Well no. 2

Screen: 137-624 feet

Altitude: 139 feet

\begin{tabular}{ll}
\hline Date & $\begin{array}{l}\text { Hater } \\
\text { level }\end{array}$ \\
\hline
\end{tabular}

03-12-69

03-02-70

03-11-71

$02-23-72$

$03-05-73$

$03-05-74$

$02-25-75$

$02-27-76$

$03-07-77$

$11-17-77$

$03-14-78$

$11-16-78$

02-08-79

12-10-79

$01-18-80$

$12-15-80$

01-15-81

10-29-81

02-01-82

$11-10-82$

01-17-83

$01-25-83$

$09-22-83$

02-02-84

$01-22-85$

$01-28-86$

$01-08-87$
97.34

99.52

101.13

102.72

101.08

102.12

108.06

109.85

108.25

102.96

97.72

95.54

95.30

95.76

95.51

96.66

96.51

96.96

93.24

96.87

94.05

94.68

96.93

97.55

91.24

89.05

88.63
Well JY-65-18-111

Owner: Cardiff Bros. Screen: 339-1,000 feet Alt $\mathrm{i}$ tude: 138 feet

\begin{tabular}{cc}
\hline Date & $\begin{array}{l}\text { Hater } \\
\text { level }\end{array}$ \\
\hline $02-14-86$ & 129.10 \\
$02-10-87$ & 128.75
\end{tabular}

Hell JY-65-18-202 Owner: Cinco Ranch Screen: 100-534 feet Altitude: 127 feet

\begin{tabular}{ll}
\hline Hater \\
Date & \begin{tabular}{l} 
level \\
\hline
\end{tabular} \\
\hline
\end{tabular}

03-12-69

93.24

03-02-70

95.40

$03-11-71$

96.06

$02-23-72$

98.06

$03-07-73$

100.24

03-05-74

100.66

$02-25-75$

102.32

$02-27-76$

103.55

03-07-77

103.95

$11-17-77$

107.72

$03-14-78$

104.72

$11-16-78$

109.26

$02-08-79$

109.61

12-07-79

112.17

$01-08-80$

111.38

$12-15-80$

112.59

$01-25-81$

112.25

10-29-81

113.61

02-01-82

$11-10-82$

113.37

115.26

$01-17-83$

114.51

09-22-83

115.70

$01-25-84$

113.04

01-23-85 108.38

01-28-86 $\quad 106.94$

01-08-87 $\quad 105.89$

Hell JY-65-18-602

Owner: E. H. Gless Screen: $120-520$ feet Altitude: 103 feet

\begin{tabular}{ll}
\hline Date & $\begin{array}{l}\text { Water } \\
\text { level }\end{array}$ \\
\hline
\end{tabular}

03-12-69

79.91

03-02-70

82.18

03-11-71

81.72

$02-23-72$

86.48

03-07-73

86.06

03-05-74

87.07

$02-25-75$

86.38

03-03-76

87.54

03-07-77

11-17-77

88.55

$03-14-78$

93.43

90.79

11-16-78

02-08-79

94.56

12-07-79

94.35

94.79

01-18-80

94.47

12-15-80

95.20

01-15-81

95.16

10-29-81

95.43

02-01-82 
Table 4.-- Hater Levels in selected wells in Fort Bend County, 1969-87--Cont inued

Well JY-65-18-602--Cont.

$\begin{array}{rr}10-26-82 & 100.10 \\ 01-17-83 & 102.04 \\ 09-22-83 & 103.17 \\ 01-25-84 & 98.16 \\ 01-23-85 & 96.44 \\ 02-06-87 & 100.21\end{array}$

Hell JY-65-18-604

Owner: Ed Helwig

Screen: $230-620$ feet

Altitude: 100 feet

\begin{tabular}{cc}
\hline Date & $\begin{array}{c}\text { Mater } \\
\text { level }\end{array}$ \\
\hline $03-03-87$ & 179.56
\end{tabular}

Hell JY-65-19-101

Owner: H. W. Wheeless

Depth: 400 feet

Altitude: 96 feet

\begin{tabular}{cc}
\hline Date & $\begin{array}{l}\text { Water } \\
\text { Level }\end{array}$ \\
\hline $02-18-86$ & 131.50 \\
$02-05-87$ & 130.02
\end{tabular}

Hell JY-65-19-509

Owner: Ft. Bend County M.U.D. 30

Screen: 635-868 feet

Altitude: 95 feet

\begin{tabular}{ll}
\hline Date & $\begin{array}{l}\text { Hater } \\
\text { level }\end{array}$ \\
\hline $04-24-79$ & 167 \\
$02-02-84$ & 162.90 \\
$01-28-85$ & 161.23 \\
$01-27-86$ & 164.22 \\
$01-07-87$ & 165.16
\end{tabular}

Well JY-65-19-704 Owner: Cinco Ranch Screen: 161-528 feet Altitude: 101 feet

\begin{tabular}{cc}
\hline \multicolumn{1}{c}{ Date } & $\begin{array}{l}\text { Water } \\
\text { level }\end{array}$ \\
\hline $03-12-69$ & 79.25 \\
$03-02-70$ & 82.23 \\
$03-11-71$ & 83.64 \\
$02-23-72$ & 86.44 \\
$03-08-73$ & 85.41 \\
$02-25-75$ & 86.24 \\
$03-03-76$ & 86.76 \\
$03-07-77$ & 89.60 \\
$11-23-77$ & 95.05 \\
$03-14-78$ & 91.82 \\
$11-16-78$ & 96.21 \\
$02-08-79$ & 95.57 \\
$12-07-79$ & 96.63 \\
$01-14-80$ & 96.48 \\
$12-15-80$ & 96.91 \\
$01-14-81$ & 96.53 \\
$10-29-81$ & 97.66 \\
$02-01-82$ & 99.27
\end{tabular}

Well JY-65-19-704--Cont.

$\begin{array}{rr}10-26-82 & 100.52 \\ 01-14-83 & 100.26 \\ 09-21-83 & 100.74 \\ 01-25-84 & 100.45 \\ 01-23-85 & 96.41 \\ 01-28-86 & 94.99 \\ 02-18-86 & 112.51 \\ 01-07-87 & 94.58 \\ 02-06-87 & 102.47\end{array}$

Hell JY-65-19-801

Owner: Texas Department of Corrections

Screen: 196-256 feet

Altitude: 92 feet

\begin{tabular}{cc}
\hline Date & $\begin{array}{l}\text { Water } \\
\text { level }\end{array}$ \\
\hline $02-08-86$ & 109.19
\end{tabular}

Hell JY-65-19-803

Owner: Texas Department of Corrections

Screen: 137-233 feet

Altitude: 85 feet

\begin{tabular}{ll}
\hline Date & $\begin{array}{l}\text { Water } \\
\text { level }\end{array}$ \\
\hline $02-08-86$ & 98.38
\end{tabular}

Hell JY-65-19-804

Owner: Texas Department of Corrections Screen: 128-231 feet

Altitude: 85 feet

\begin{tabular}{cc}
\hline Date & $\begin{array}{l}\text { Mater } \\
\text { level }\end{array}$ \\
\hline $02-08-86$ & 81.15
\end{tabular}

Well JY-65-19-902

Owner: Texas Department of Corrections

Screen:

Altitude: 87 feet

\begin{tabular}{ll}
\hline Date & $\begin{array}{l}\text { Hater } \\
\text { Level }\end{array}$ \\
\hline $03-14-86$ &
\end{tabular}

03-14-86 $\quad 16.86$

Hell JY-65-20-702

Owner: Flopetrol Johnston

Screen: 823-1006 feet

Altitude: 83 feet

\begin{tabular}{ll}
\hline Date & $\begin{array}{l}\text { Water } \\
\text { level }\end{array}$ \\
\hline
\end{tabular}

03-19-86 258.10
Well JY-65-25-201

Owner: Duval Sulphur and Potash Co. Hell no. 52

Screen: 144-284 feet

Altitude: 115 feet

\begin{tabular}{cc}
\hline Date & $\begin{array}{l}\text { Mater } \\
\text { level }\end{array}$ \\
\hline $02-13-86$ & 39.23 \\
$02-16-87$ & 39.77
\end{tabular}

Hell JY-65-25-202

Owner: Duval Sulphur and Potash Co., Hell no. 48

Screen: 120-279 feet Altitude: 115 feet

\begin{tabular}{cc}
\hline Date & $\begin{array}{l}\text { Hater } \\
\text { level }\end{array}$ \\
\hline $02-13-86$ & 49.13 \\
$02-16-87$ & 49.50
\end{tabular}

Well JY-65-25-203

Owner: Duval Sulphur and Potash Co., Hell no. 46

Screen: 151-276 feet Altitude: 115 feet

\begin{tabular}{cc}
\hline Hater \\
level
\end{tabular}

$01-18-72$ 45.07

08-08-72 47.37

$01-23-73$

$03-17-78$

08-08-78

$02-21-79$

08-02-79

$01-15-80$

08-06-80

01-26-81

47.37
47.80

46.14

49.86

46.12

46.49

40.91

50.31

45.16
51.83

02-17-82 $\quad 40.73$

$02-02-83 \quad 48.20$

08-10-83 $\quad 49.42$

$01-27-84 \quad 48.75$

08-02-84 $\quad 49.56$

$01-21-85 \quad 47.88$

$02-13-86 \quad 44.98$

02-16-87 $\quad 46.62$

Well JY-65-25-301 Owner: R. E. Smith Screen: $91-432$ feet Alt itude: 111 feet

\begin{tabular}{cc}
\hline Date & $\begin{array}{l}\text { Hater } \\
\text { level }\end{array}$ \\
\hline $01-14-69$ & 50.33 \\
$08-11-69$ & 51.83 \\
$01-30-70$ & 50.97 \\
$01-11-71$ & 51.47 \\
$01-17-72$ & 49.66 \\
$08-08-72$ & 51.55 \\
$01-17-73$ & 50.39 \\
$01-16-75$ & 46.51
\end{tabular}


Hell JY-65-25-301--Cont.

$\begin{array}{ll}01-13-76 & 52.29 \\ 03-17-78 & 50.22 \\ 03-01-79 & 51.98 \\ 08-02-79 & 49.76 \\ 01-15-80 & 46.41 \\ 01-26-81 & 52.12 \\ 08-11-81 & 53.47 \\ 02-17-82 & 51.39 \\ 07-19-82 & 54.45 \\ 02-02-83 & 54.28 \\ 08-10-83 & 55.19 \\ 01-27-84 & 51.64 \\ 08-02-84 & 56.87 \\ 01-21-85 & 52.07 \\ 09-18-85 & 61.38 \\ 02-03-86 & 57.56 \\ 02-17-87 & 58.15\end{array}$

Well JY-65-25-302 Owner: R. E. Smith Screen: 111-431 feet Altitude: 113 feet

\begin{tabular}{cc}
\hline Date & $\begin{array}{c}\text { Water } \\
\text { Level }\end{array}$ \\
\hline $01-14-69$ & 51.74 \\
$08-11-69$ & 55.07 \\
$01-30-70$ & 52.58 \\
$08-13-70$ & 52.05 \\
$01-11-71$ & 50.54 \\
$01-17-72$ & 50.29 \\
$01-17-73$ & 50.87 \\
$03-17-78$ & 51.62 \\
$03-01-79$ & 51.68 \\
$08-23-79$ & 51.70 \\
$01-15-80$ & 47.43 \\
$08-06-80$ & 56.17 \\
$01-26-81$ & 52.51 \\
$08-11-81$ & 54.17
\end{tabular}

Well JY-65-25-402 Owner: Jerry Kulhanek Depth: 245 feet Al ti tude: 120 feet

\begin{tabular}{ll}
\hline Date & $\begin{array}{l}\text { Water } \\
\text { level }\end{array}$ \\
\hline $02-20-86$ & 48.37 \\
$02-16-87$ & 48.54
\end{tabular}

Well JY-65-25-701 Owner: Herman and Lloyd Engle Screen: 98-284 feet Al titude: 115 feet

\begin{tabular}{ll}
\hline Date & $\begin{array}{l}\text { Water } \\
\text { Level }\end{array}$ \\
\hline $02-20-86$ & 51.51 \\
$02-18-87$ & 50.33
\end{tabular}

Well JY-65-25-703

Owner: August Blazek

Depth: 295 feet

Al $t$ i tude: 114 feet

\begin{tabular}{ll}
\hline Date & Hater \\
level
\end{tabular}

02-20-86

47.45
Hell JY-65-25-709

Owner: Lloyd Engle

Screen: $96-305$ feet

Altitude: 108 feet

\begin{tabular}{ll}
\hline Date & \multicolumn{1}{c}{$\begin{array}{l}\text { Water } \\
\text { level }\end{array}$} \\
\hline $02-20-86$ & 49.98 \\
$02-16-87$ & 48
\end{tabular}

Hell JY-65-25-801

Owner:

Screen:

Altitude: 111 feet

\begin{tabular}{ll}
\hline Date & $\begin{array}{l}\text { Water } \\
\text { level }\end{array}$ \\
\hline $02-20-86$ & 54.39
\end{tabular}

Well JY-65-26-201

Owner: R. E. Smith

Screen: 200-575 feet

Altitude: 90 feet

\begin{tabular}{cc}
\hline Date & $\begin{array}{l}\text { Water } \\
\text { level }\end{array}$ \\
\hline $03-07-86$ & 66.30
\end{tabular}

Well JY-65-26-202

Owner: Dickerson

Depth: 305 feet

Altitude: 89 feet

\begin{tabular}{ll}
\hline Date & $\begin{array}{l}\text { Water } \\
\text { level }\end{array}$ \\
\hline $03-07-86$ & 63.78 \\
$02-10-87$ & 63.66
\end{tabular}

Hell JY-65-26-403

Owner: Gulf States

Depth: 875 feet

Altitude: 103 feet

\begin{tabular}{|c|c|}
\hline Date & $\begin{array}{l}\text { Water } \\
\text { level }\end{array}$ \\
\hline $\begin{array}{l}03-07-86 \\
02-16-87\end{array}$ & $\begin{array}{r}100.40 \\
92.99\end{array}$ \\
\hline \multicolumn{2}{|c|}{$\begin{array}{l}\text { Well JY-65-26-406 } \\
\text { Owner: Gulf States } \\
\text { Depth: } 1,178 \text { feet } \\
\text { Al ti tude: } 103 \text { feet }\end{array}$} \\
\hline Date & $\begin{array}{l}\text { Water } \\
\text { level }\end{array}$ \\
\hline $\begin{array}{l}03-07-86 \\
02-16-87\end{array}$ & $\begin{array}{l}136.20 \\
135.35\end{array}$ \\
\hline
\end{tabular}

Well JY-65-26-501

Owner: City of Rosenberg, Hell no. 3 Screen: $545-837$ feet Altitude: 103 feet

\begin{tabular}{cc}
\hline Date & $\begin{array}{l}\text { Water } \\
\text { Level }\end{array}$ \\
\hline $02-27-86$ & 108.50
\end{tabular}

Hell JY-65-26-502

Owner: City of Rosenberg, Well no. 4 Screen: 629-966 feet Altitude: 103 feet

\begin{tabular}{cc}
\hline Date & $\begin{array}{l}\text { Hater } \\
\text { level }\end{array}$ \\
\hline $02-18-87$ & 108.10
\end{tabular}

Well JY-65-26-601 Owner: City of Richmond, Hell no. 3 Screen: $317-447$ feet Altitude: 95 feet

\begin{tabular}{ll}
\hline Date & $\begin{array}{l}\text { Water } \\
\text { level }\end{array}$ \\
\hline $03-24-86$ & 88.20
\end{tabular}

Well JY-65-26-603 Owner: City of Richmond Hell no. 4 Screen: $342-514$ feet Altitude: 90 feet

\begin{tabular}{ll}
\hline Date & $\begin{array}{l}\text { Water } \\
\text { level }\end{array}$ \\
\hline $03-24-86$ & 98.50
\end{tabular}

Hell JY-65-26-812

Owner: City of Rosenberg, Well no. 6

Screen: $810-1,310$ feet Altitude: 99 feet

\begin{tabular}{cc}
\hline Date & $\begin{array}{l}\text { Water } \\
\text { level }\end{array}$ \\
\hline $02-18-87$ & 135.50
\end{tabular}

Well JY-65-27-202

Owner: Texas Department of Corrections, Harlem, no. 3

Screen: $43-90$ feet Altitude: 80 feet

\begin{tabular}{cc}
\hline Date & $\begin{array}{l}\text { Water } \\
\text { Level }\end{array}$ \\
\hline $01-21-86$ & 16.26
\end{tabular}


Well JY-65-27-203

Owner: R. E. Smith

Screen: 26-72 feet

Altitude: 79 feet

\begin{tabular}{cc}
\hline Date & $\begin{array}{l}\text { Water } \\
\text { level }\end{array}$ \\
\hline $01-21-86$ & 21.39
\end{tabular}

Well JY-65-27-204

Owner: Texas Department of Corrections, Harlem, no. 1

Screen: 42-91 feet

Altitude: 83 feet

\begin{tabular}{cc}
\hline Date & $\begin{array}{l}\text { Water } \\
\text { level }\end{array}$ \\
\hline $01-21-86$ & 16.26
\end{tabular}

Well JY-65-27-205

Owner: Texas Department of Corrections, Harlem, no. 4

Screen: 47-86 feet

Altitude: 84 feet

\begin{tabular}{cc}
\hline Date & $\begin{array}{l}\text { Water } \\
\text { level }\end{array}$ \\
\hline $01-21-86$ & 11.81
\end{tabular}

Well JY-65-27-207

Owner: Texas Department of Corrections, Central Unit no.7

Screen: 25-62 feet

Altitude: 80 feet

\begin{tabular}{ll}
\hline Date & $\begin{array}{l}\text { Water } \\
\text { level }\end{array}$ \\
\hline $01-21-86$ &
\end{tabular}

Well JY-65-27-302

Owner: Ft. Bend Utilities, Well no. 8

Screen: $1,260-1,560$ feet

Altitude: 80 feet

\begin{tabular}{cl}
\hline Date & $\begin{array}{l}\text { Water } \\
\text { level }\end{array}$ \\
\hline $12-13-69$ & 187 \\
$01-30-71$ & 188 \\
$01-30-72$ & 212 \\
$10-21-72$ & 217 \\
$01-13-73$ & 221 \\
$07-21-73$ & 222 \\
$04-09-74$ & 209 \\
$05-19-74$ & 226 \\
$11-03-74$ & 215 \\
$05-03-75$ & 213 \\
$08-16-75$ & 217 \\
$02-06-76$ & 218 \\
$06-12-76$ & 217 \\
$08-20-76$ & 202 \\
$12-07-76$ & 207 \\
$03-28-77$ & 202 \\
$07-18-77$ & 207 \\
$01-24-78$ & 217 \\
$08-19-78$ & 232
\end{tabular}

Well JY-65-27-302--Cont.

$\begin{array}{ll}11-08-79 & 227 \\ 03-01-80 & 227 \\ 11-18-80 & 244 \\ 02-01-81 & 245 \\ 11-06-81 & 267 \\ 01-27-82 & 267 \\ 05-28-82 & 264 \\ 09-16-82 & 282 \\ 11-30-82 & 282 \\ 01-10-83 & 290 \\ 06-29-84 & 287 \\ 09-10-84 & 282 \\ 08-20-85 & 292 \\ 01-15-86 & 292 \\ 12-30-86 & 282\end{array}$

Well JY-65-27-303

Owner: Ft. Bend Utilities, Well no. 9

Screen: 503-865 feet

Altitude: 80 feet

\begin{tabular}{ll}
\hline Date & $\begin{array}{c}\text { Water } \\
\text { level }\end{array}$ \\
\hline $12-13-69$ & 123 \\
$01-30-71$ & 126 \\
$01-30-72$ & 145 \\
$10-21-72$ & 150 \\
$01-13-73$ & 146 \\
$01-21-73$ & 146 \\
$01-04-74$ & 148 \\
$05-19-74$ & 147 \\
$11-03-74$ & 156 \\
$05-03-75$ & 145 \\
$08-16-75$ & 153 \\
$02-06-76$ & 153 \\
$06-12-76$ & 163 \\
$08-20-76$ & 161 \\
$12-07-76$ & 160 \\
$03-28-77$ & 158 \\
$07-18-77$ & 168 \\
$01-24-78$ & 173 \\
$08-19-78$ & 173 \\
$11-08-79$ & 178 \\
$03-01-80$ & 168 \\
$11-18-80$ & 184 \\
$02-01-81$ & 174 \\
$11-06-81$ & 168 \\
$01-27-82$ & 186 \\
$05-28-82$ & 183 \\
$09-16-82$ & 198 \\
$11-30-82$ & 195 \\
$01-10-83$ & 193 \\
$11-04-83$ & 193 \\
$01-26-84$ & 193 \\
$09-10-84$ & 202 \\
$01-10-85$ & 198 \\
$08-20-85$ & 213 \\
$01-15-86$ & 203 \\
$12-30-86$ & 203 \\
&
\end{tabular}

Well JY-65-27-305

Owner: Texas Department of Corrections, Central Unit no. 3

Screen: 27-72 feet

Altitude: 73 feet

\begin{tabular}{cc}
\hline Date & $\begin{array}{l}\text { Water } \\
\text { level }\end{array}$ \\
\hline $01-21-86$ & 34.90
\end{tabular}

Well JY-65-27-306

Owner: Texas Department of Corrections, Central Unit no. 5

Screen: 55-100 feet

Altitude: 73 feet

\begin{tabular}{cc}
\hline Date & $\begin{array}{l}\text { Water } \\
\text { Level }\end{array}$ \\
\hline $01-21-86$ & 20
\end{tabular}

Well JY-65-27-307

Owner: Texas Department of Corrections, Central Unit no. 8

Screen: $53-83$ feet

Altitude: 73 feet

\begin{tabular}{cc}
\hline Date & $\begin{array}{l}\text { Water } \\
\text { level }\end{array}$ \\
\hline $01-21-86$ & 17.28
\end{tabular}

Well JY-65-27-308

Owner: Texas Department of Corrections Central Unit no. 11 Screen: 46-104 feet Altitude: 76 feet

\begin{tabular}{cc}
\hline Date & $\begin{array}{l}\text { Hater } \\
\text { level }\end{array}$ \\
\hline $01-22-86$ & 17.69
\end{tabular}

Well JY-65-27-313

Owner: Ft. Bend Utilities: Hell no. 7

Screen: 501-721 feet

Altitude: 77 feet

\begin{tabular}{ll}
\hline Date & $\begin{array}{l}\text { Water } \\
\text { level }\end{array}$ \\
\hline $01-30-72$ & 153 \\
$10-21-72$ & 152 \\
$01-13-73$ & 148 \\
$07-21-73$ & 152 \\
$01-04-74$ & 149 \\
$05-19-74$ & 151 \\
$11-03-74$ & 157 \\
$08-16-75$ & 155 \\
$02-06-76$ & 151 \\
$06-12-76$ & 152 \\
$08-20-76$ & 161 \\
$12-07-76$ & 161 \\
$03-28-77$ & 157 \\
$07-18-77$ & 169 \\
$01-24-78$ & 169 \\
$08-19-78$ & 174 \\
$03-01-80$ & 174 \\
$11-18-80$ & 184 \\
$02-01-81$ & 181 \\
$11-06-81$ & 190 \\
$01-27-82$ & 184 \\
$05-28-82$ & 183 \\
$09-16-82$ & 196 \\
$11-30-82$ & 194 \\
$01-10-83$ & 192 \\
$06-29-84$ & 206 \\
&
\end{tabular}


Well JY-65-27-313--Cont.

$\begin{array}{ll}09-10-84 & 206 \\ 01-10-85 & 199 \\ 08-20-85 & 211 \\ 01-15-86 & 204 \\ 12-30-86 & 203\end{array}$

Well JY-65-27-601

Owner: Texas Department of Corrections, Central Unit no. 4

Screen: 41-86 feet

Altitude: 73 feet

\begin{tabular}{ll}
\hline Date & $\begin{array}{l}\text { Water } \\
\text { level }\end{array}$ \\
\hline $01-22-86$ & 30.17
\end{tabular}

Well JY-65-27-602

Owner: Texas Department of Corrections, Central Unit

no. 9

Screen: $48-83$ feet

Altitude: 73 feet

\begin{tabular}{ll}
\hline Date & $\begin{array}{l}\text { Water } \\
\text { level }\end{array}$ \\
\hline $01-21-86$ & 31.50
\end{tabular}

Well JY-65-27-603

Owner: Texas Department of Corrections, Central Unit no. 1

Screen: 33-78 feet

Al ti tude: 71 feet

\begin{tabular}{ll}
\hline Date & $\begin{array}{l}\text { Water } \\
\text { level }\end{array}$ \\
\hline $01-22-86$ & 29.89
\end{tabular}

Well JY-65-27-607

Owner: Agnes Booth

Depth: 200 feet

Al titude: 70 feet

\begin{tabular}{cc}
\hline Date & $\begin{array}{l}\text { Water } \\
\text { level }\end{array}$ \\
\hline $01-22-86$ & 30.17
\end{tabular}

Well JY-65-27-901

Owner: A. E. Myer

Screen: 633-673 feet

Altitude: 74 feet

\begin{tabular}{ll}
\hline Date & $\begin{array}{l}\text { Water } \\
\text { level }\end{array}$ \\
\hline $01-23-86$ & 149.33 \\
$02-05-87$ & 147.85
\end{tabular}

Well JY-65-28-201

Owner: Ft. Bend County W.C.1.D. 2, Well no. 1

Screen: 569-680 feet

Altitude: 82 feet

\begin{tabular}{ll}
\hline Date & $\begin{array}{l}\text { Water } \\
\text { level }\end{array}$ \\
\hline $03-02-87$ & 251.85
\end{tabular}

Well JY-65-28-202

Owner: Ft. Bend County W.C.I.D. 2, Well no. 2

Screen: $1,120-1,670$ feet

Altitude: 82 feet

\begin{tabular}{ll}
\hline Date & $\begin{array}{l}\text { Water } \\
\text { level }\end{array}$ \\
\hline $03-02-87$ & 284
\end{tabular}

Well JY-65-28-308

Owner: United Gas Co.

Screen: 280-300 feet

Altitude: 72 feet

\begin{tabular}{ll}
\hline Date & $\begin{array}{l}\text { Water } \\
\text { level }\end{array}$ \\
\hline $02-04-87$ & 137.30
\end{tabular}

Well JY-65-28-309

Owner: Blue Ridge M.U.D., Chasewood, Well no. 1

Screen: $770-1,020$ feet

Altitude: 70 feet

\begin{tabular}{ll}
\hline Date & $\begin{array}{l}\text { Water } \\
\text { level }\end{array}$ \\
\hline $05-31-69$ & 189 \\
$01-31-84$ & 243.18 \\
$01-28-85$ & 241.94 \\
$01-27-86$ & 245.16 \\
$01-07-87$ & 246.57
\end{tabular}

Well JY-65-28-311

Owner: City of Houstion,

Sims Bayou, Well no. 6

Screen: $656-1,182$ feet

Altitude: 67 feet

\begin{tabular}{cc}
\hline Date & $\begin{array}{c}\text { Water } \\
\text { level }\end{array}$ \\
\hline $12-12-74$ & 218.48 \\
$02-04-75$ & 215.18 \\
$02-05-76$ & 215.68 \\
$02-04-77$ & 220.49 \\
$02-22-78$ & 252.13 \\
$01-23-79$ & 267.54 \\
$01-30-80$ & 270.97 \\
$01-12-81$ & 287.19 \\
$01-19-82$ & 289.67 \\
$01-07-83$ & 291.74 \\
$01-17-84$ & 290.36 \\
$01-15-85$ & 320.20 \\
$01-27-86$ & 323.65 \\
$01-07-87$ & 325.02
\end{tabular}

Well JY-65-28-313

Owner: Ft. Bend County M.U.D. 26

Screen: $800-1,180$ feet Alt $i$ tude: 80 feet

\begin{tabular}{cl}
\hline Date & $\begin{array}{l}\text { Water } \\
\text { level }\end{array}$ \\
\hline $06-30-80$ & 274 \\
$01-31-84$ & 273.74 \\
$01-28-85$ & 271.95 \\
$03-06-86$ & 285.67 \\
$01-07-87$ & 276.72 \\
$02-11-87$ & 271.50
\end{tabular}

Well JY-65-28-401

Owner: Exxon

Screen: $684-710$ feet

Altitude: 68 feet

\begin{tabular}{cc}
\hline Date & $\begin{array}{l}\text { Water } \\
\text { level }\end{array}$ \\
\hline $02-12-86$ & 183.64 \\
$02-04-87$ & 181.78
\end{tabular}

Well JY-65-28-501

Owner: Exxon

Screen: $426-446$ feet

Altitude: 67 feet

\begin{tabular}{ll}
\hline Date & $\begin{array}{l}\text { Water } \\
\text { level }\end{array}$ \\
\hline $01-23-69$ & 120.40 \\
$08-12-69$ & 122.17 \\
$01-30-70$ & 124.7 \\
$08-13-70$ & 126.8 \\
$01-11-71$ & 128 \\
$08-02-71$ & 133.4 \\
$01-17-72$ & 135 \\
$08-07-72$ & 138.8 \\
$01-16-73$ & 136.81 \\
$08-13-73$ & 137.5 \\
$08-07-74$ & 143.5 \\
$01-15-75$ & 137.8 \\
$08-08-75$ & 143 \\
$01-12-76$ & 146 \\
$08-04-76$ & 146.6 \\
$01-12-77$ & 145.2 \\
$08-02-77$ & 155.6 \\
$03-16-78$ & 156.64 \\
$08-08-78$ & 160.92 \\
$02-21-79$ & 166 \\
$03-01-79$ & 165.69 \\
$08-06-79$ & 166.96 \\
$01-15-80$ & 164.71 \\
$02-04-87$ & 178
\end{tabular}

Well JY-65-28-508 Owner: Quail Valley U.D., Well no. 2 Screen: $752-1,300$ feet Al titude: 70 feet

\begin{tabular}{ll}
\hline \multicolumn{1}{c}{ Date } & \multicolumn{1}{c}{$\begin{array}{l}\text { Water } \\
\text { level }\end{array}$} \\
\hline $05--78$ & 214 \\
$01-31-84$ & 233.13 \\
$01-28-85$ & 230.79 \\
$02-14-86$ & 237.40 \\
$03-06-86$ & 249.43 \\
$02-11-87$ & 236
\end{tabular}


Table 4.-- Hater levels in selected wells in Fort Bend County, 1969-87--Cont inued

Hell JY-65-28-803

Owner: Christianson and Mat thews

Depth: 420 feet

Altitude: 60 feet

\begin{tabular}{cc}
\hline Date & $\begin{array}{c}\text { Water } \\
\text { level }\end{array}$ \\
\hline $01-27-69$ & 62.89 \\
$01-30-70$ & 64.54 \\
$01-14-71$ & 65.08 \\
$01-26-72$ & 66.83 \\
$01-19-73$ & 68.32 \\
$01-16-74$ & 67.84 \\
$01-16-75$ & 66.92 \\
$01-12-76$ & 68.46 \\
$03-16-78$ & 73.36 \\
$03-01-79$ & 75.85 \\
$08-06-79$ & 74.14 \\
$01-15-80$ & 77 \\
$08-06-80$ & 78.08 \\
$01-26-81$ & 78.13 \\
$08-11-81$ & 80.13 \\
$03-01-82$ & 78.76 \\
$07-21-82$ & 82.67 \\
$02-02-83$ & 84.26 \\
$08-10-83$ & 81.10 \\
$01-30-84$ & 82 \\
$08-02-84$ & 83.87 \\
$01-29-87$ & 90.08
\end{tabular}

Well JY-65-29-101

Owner: D. H. Black

Screen: $240-800$ feet

Altitude: 70 feet

\begin{tabular}{cc}
\hline Date & $\begin{array}{c}\text { Water } \\
\text { level }\end{array}$ \\
\hline $02-09-70$ & 126.75 \\
$02-09-71$ & 129.42 \\
$02-01-72$ & 134.27 \\
$01-17-73$ & 135.27 \\
$01-17-74$ & 130.34
\end{tabular}

Well JY-65-29-104

Owner: City of Houston, Mayfair Park

Screen: 735-895 feet

Altitude: 65 feet

\begin{tabular}{cl}
\hline Date & $\begin{array}{c}\text { Hater } \\
\text { level }\end{array}$ \\
\hline $01-09-69$ & 191.14 \\
$01-16-73$ & 212.51 \\
$08-14-73$ & 216.15 \\
$01-17-74$ & 212.67 \\
$08-07-74$ & 221.72 \\
$01-16-75$ & 218.20 \\
$08-12-75$ & 227.93 \\
$01-12-76$ & 231.96 \\
$08-04-76$ & 228.06 \\
$08-06-79$ & 276.52 \\
$08-10-83$ & 333 \\
$01--87$ & 298
\end{tabular}

Hell JY-65-29-405

Owner: Blue Ridge Elementary School

Screen: 518-553 feet

Altitude: 72 feet

\begin{tabular}{ll}
\hline Date & \multicolumn{1}{c}{$\begin{array}{l}\text { Hater } \\
\text { level }\end{array}$} \\
\hline $02-07-86$ & 258.15 \\
$01-28-87$ & 253
\end{tabular}

Hell JY-65-29-407

Owner: Houston Gun club

Screen: 90-100 feet

Altitude: 95 feet

Altitude: 68 feet

\begin{tabular}{ll}
\hline Date & $\begin{array}{l}\text { Hater } \\
\text { level }\end{array}$ \\
\hline $02-07-86$ & 14.10
\end{tabular}

Well JY-65-29-701

Owner: Jul ia Tague

Depth: 459 feet

Alt itude: 65 feet

\begin{tabular}{|c|c|}
\hline Date & $\begin{array}{l}\text { Hater } \\
\text { level }\end{array}$ \\
\hline $\begin{array}{l}01-27-69 \\
01-21-70 \\
01-14-71 \\
08-12-71 \\
01-26-72 \\
08-11-72 \\
01-16-73 \\
08-10-73 \\
01-16-74 \\
08-09-74 \\
01-16-75 \\
01-26-76 \\
08-04-76 \\
01-17-77 \\
08-03-77 \\
03-21-78 \\
08-09-78 \\
02-28-79 \\
08-06-79 \\
01-17-80 \\
08-06-80 \\
01-26-81 \\
08-12-81 \\
02-23-82 \\
07-21-82 \\
02-01-83 \\
08-10-83 \\
02-21-84 \\
08-02-84 \\
01-21-85 \\
10-01-85 \\
02-03-86\end{array}$ & $\begin{array}{r}78.87 \\
81.61 \\
83.31 \\
84.23 \\
84.96 \\
86.42 \\
86.50 \\
87.02 \\
86.69 \\
87.23 \\
87.45 \\
88.84 \\
89.34 \\
86.24 \\
90.22 \\
91.77 \\
95.13 \\
94.83 \\
97 \\
94.28 \\
94.18 \\
94.12 \\
94.30 \\
99.82 \\
98.49 \\
100.85 \\
104.76 \\
102.13 \\
105.53 \\
106 \\
108.61 \\
108.62\end{array}$ \\
\hline \multicolumn{2}{|c|}{$\begin{array}{l}\text { Hell JY-65-33-101 } \\
\text { Owner: Kubala } \\
\text { Depth: } 277 \text { feet } \\
\text { Al t i tude: } 107 \text { feet }\end{array}$} \\
\hline Date & $\begin{array}{l}\text { Water } \\
\text { level }\end{array}$ \\
\hline
\end{tabular}

Hell JY-65-33-102

Owner: Ed Stasney Depth: 259 feet

Alt itude: 101 feet

\begin{tabular}{cc}
\hline Date & $\begin{array}{l}\text { Hater } \\
\text { level }\end{array}$ \\
\hline $01-13-86$ & 44.49
\end{tabular}

Well JY-65-33-103

Owner: E. Pitts

Depth: 301 feet

Altitude: 101 feet

\begin{tabular}{cc}
\hline Date & $\begin{array}{l}\text { Hater } \\
\text { level }\end{array}$ \\
\hline $01-13-86$ & 44.64
\end{tabular}

Hell JY-65-33-501

Owner: Jack Wendt, Well no. 4

Screen: 126-376 feet Altitude: 97 feet

\begin{tabular}{cc}
\hline Date & $\begin{array}{l}\text { Hater } \\
\text { level }\end{array}$ \\
\hline $01-23-69$ & 42.64 \\
$01-29-70$ & 43.66 \\
$01-12-71$ & 43.83 \\
$01-17-72$ & 44.74 \\
$01-17-73$ & 44.98 \\
$01-17-74$ & 44.83 \\
$01-20-75$ & 44.96 \\
$01-19-76$ & 44.83 \\
$01-17-77$ & 45.37 \\
$03-20-78$ & 46.14 \\
$02-22-79$ & 46.21 \\
$08-23-79$ & 52.10 \\
$01-16-80$ & 46.02 \\
$01-26-81$ & 47.16 \\
$08-12-81$ & 48.44 \\
$07-23-82$ & 50.77 \\
$02-21-84$ & 52.16
\end{tabular}

Hell JY-65-33-502

Owner: Jack Wendt Depth: 590 feet Altitude: 95 feet

\begin{tabular}{ll}
\hline Date & $\begin{array}{l}\text { Hater } \\
\text { level }\end{array}$ \\
\hline $01-17-73$ & 40 \\
$01-17-74$ & 39.48 \\
$01-20-75$ & 39.62 \\
$01-14-76$ & 40 \\
$01-17-77$ & 42.56 \\
$03-20-78$ & 40.73 \\
$02-22-79$ & 41.32 \\
$08-23-79$ & 43.99 \\
$01-16-80$ & 40.29 \\
$08-07-80$ & 45.75 \\
$01-26-81$ & 40.89 \\
$08-12-81$ & 45.13 \\
$02-24-82$ & 44.31 \\
$07-23-82$ & 46.11 \\
$02-03-83$ & 45.91 \\
$09-01-83$ & 47.37 \\
$02-21-84$ & 48.61 \\
$01-21-85$ & 46.20
\end{tabular}


Table 4.-- Water levels in selected wells in Fort Bend County, 1969-87---Cont inued

Well JY-65-33-502---Cont.

$\begin{array}{ll}11-14-85 & 47.40 \\ 01-14-86 & 45.83 \\ 03-20-87 & 45.55\end{array}$

Well JY-65-33-503

Owner: Jack Wendt,

Depth: 240 feet

Alt itude: 95 feet

\begin{tabular}{ll}
\hline Date & $\begin{array}{l}\text { Water } \\
\text { level }\end{array}$ \\
\hline $01-23-69$ & 40.98 \\
$01-29-70$ & 41.27 \\
$01-12-71$ & 41.47 \\
$01-17-72$ & 42.17 \\
$01-20-75$ & 44.26 \\
$01-17-77$ & 46.10 \\
$03-20-78$ & 46 \\
$02-22-79$ & 48 \\
$01-16-80$ & 46 \\
$01-26-81$ & 47.5 \\
$08-12-81$ & 49.8 \\
$02-24-82$ & 48.4 \\
$07-23-82$ & 50.5 \\
$02-21-84$ & 49.7 \\
$01-21-85$ & 49.01 \\
$11-14-85$ & 50 \\
$01-14-86$ & 50
\end{tabular}

Well JY-65-33-504

Owner: Jack Wendt Well no. 1

Screen: $112-397$ feet

Altitude: 95 feet

\begin{tabular}{cc}
\hline Date & $\begin{array}{l}\text { Water } \\
\text { level }\end{array}$ \\
\hline $01-23-69$ & 40.70 \\
$01-29-70$ & 42.01 \\
$01-12-71$ & 42.13 \\
$01-17-72$ & 43.11 \\
$01-17-73$ & 43.04 \\
$01-17-74$ & 43.74 \\
$01-20-75$ & 42.91 \\
$01-19-76$ & 43.03 \\
$01-17-77$ & 43.42 \\
$03-20-78$ & 43.40 \\
$02-22-79$ & 43.48 \\
$08-23-79$ & 48.52 \\
$01-16-80$ & 43.36 \\
$08-07-80$ & 54.55 \\
$01-26-81$ & 44.51 \\
$08-12-81$ & 49.63 \\
$02-24-82$ & 46.22 \\
$07-23-82$ & 53.54 \\
$02-03-83$ & 53.28 \\
$09-01-83$ & 54.33 \\
$02-21-84$ & 54.93 \\
$01-21-85$ & 47.96 \\
$11-14-85$ & 53.37 \\
$01-14-86$ & 47.50 \\
$02-18-87$ & 47.70
\end{tabular}

Well JY-65-33-509

Owner: Jack Wendt

Screen: 120-623 feet

Altitude: 96 feet

\begin{tabular}{cl}
\hline Date & $\begin{array}{l}\text { Water } \\
\text { level }\end{array}$ \\
\hline $03-24-71$ & 54 \\
$01-17-73$ & 45.23 \\
$01-17-74$ & 43.80 \\
$03-20-78$ & 45.40 \\
$02-22-79$ & 50.77 \\
$08-02-79$ & 52.15 \\
$01-16-80$ & 50.16 \\
$08-07-80$ & 55.04 \\
$01-26-81$ & 51.63 \\
$08-12-81$ & 54.19 \\
$02-24-82$ & 57.32 \\
$07-23-82$ & 57.64 \\
$02-03-83$ & 53.50 \\
$09-01-83$ & 60.27 \\
$02-21-84$ & 57.29 \\
$01-21-85$ & 54.07 \\
$01-15-86$ & 54.14 \\
\end{tabular}

Well JY-65-33-510

Owner: Jack Wendt

Screen: 170-591 feet

Altitude: 95 feet

\begin{tabular}{ll}
\hline & Water \\
\hline Date & level \\
\hline $04-25-72$ & 52 \\
$01-17-73$ & 41.59 \\
$01-17-74$ & 41.13 \\
$01-20-75$ & 41.68 \\
$01-14-76$ & 37.10 \\
$01-17-77$ & 41.15 \\
$03-20-78$ & 39.48 \\
$02-22-79$ & 42.88 \\
$08-02-79$ & 46.77 \\
$01-16-80$ & 41.16 \\
$08-07-80$ & 47.10 \\
$01-26-81$ & 42.13 \\
$08-12-81$ & 48.75 \\
$02-24-82$ & 51.85 \\
$07-23-82$ & 50.91 \\
$02-03-83$ & 51.82 \\
$09-01-83$ & 52.92 \\
$02-21-84$ & 53.14 \\
$01-21-85$ & 51 \\
$11-14-85$ & 50.46 \\
$01-14-86$ & 51.69 \\
$03-20-87$ & 45.03 \\
& \\
Well JY-65-33-801 \\
Owner: Jack Hendt \\
Screen: & Well no. $317-502$ feet \\
Altitude: & 92 \\
\hline & feet \\
\hline Date & Hater \\
\hline $01-23-69$ & level \\
$01-29-70$ & 41.63 \\
$01-12-71$ & 42.70 \\
$01-17-72$ & 43.71 \\
$01-17-73$ & 43.98 \\
$01-20-75$ & 43.67 \\
$01-19-76$ & 43.39 \\
$01-17-77$ & 43.99 \\
$03-20-78$ & 44.07 \\
&
\end{tabular}

Hell JY-65-33-801--Cont.

$\begin{array}{ll}02-22-79 & 44.75 \\ 08-23-79 & 50.99 \\ 01-16-80 & 44.07 \\ 08-07-80 & 54.35 \\ 01-26-81 & 44.55 \\ 08-12-81 & 49.89 \\ 02-24-82 & 49.42 \\ 07-23-82 & 52.38 \\ 02-03-83 & 48.28 \\ 09-01-83 & 49.38 \\ 02-21-84 & 49.87 \\ 01-21-85 & 48 \\ 01-14-86 & 47.80 \\ 03-20-87 & 47.81\end{array}$

Hell JY-65-34-301

Owner: Max Mahlmann

Depth: 314 feet

Altitude: 85 feet

\begin{tabular}{ll}
\hline Date & $\begin{array}{l}\text { Hater } \\
\text { level }\end{array}$ \\
\hline $01-27-86$ & 46.05
\end{tabular}

Hell JY-65-34-303

Owner: Max Mahlmann

Screen: $100-356$ feet

Altitude: 78 feet

\begin{tabular}{ll}
\hline Date & Hater \\
level
\end{tabular}

Well JY-65-34-308

Owner: Max Mahlmann

Screen: 100-375 feet

Altitude: 86 feet

\begin{tabular}{cc}
\hline Date & $\begin{array}{l}\text { Water } \\
\text { level }\end{array}$ \\
\hline $01-29-86$ & 43.98
\end{tabular}

Hell JY-65-34-604

Owner: H. H. Gless

Screen: 220-660 feet

Altitude: 74 feet

\begin{tabular}{cc}
\hline Date & $\begin{array}{l}\text { Water } \\
\text { level }\end{array}$ \\
\hline $01-23-69$ & 60.13 \\
$01-29-70$ & 62.32 \\
$01-11-71$ & 61.58 \\
$01-17-72$ & 62.49 \\
$01-18-73$ & 63.24 \\
$01-16-74$ & 65.44 \\
$01-17-75$ & 62.03 \\
$01-14-76$ & 64.67 \\
$01-17-77$ & 63.53 \\
$03-20-78$ & 64.29 \\
$02-22-79$ & 66.95 \\
$08-03-79$ & 82.94 \\
$01-17-80$ & 66.07 \\
$08-07-80$ & 86.62 \\
$01-26-81$ & 71.90 \\
$08-11-81$ & 84.84 \\
$02-23-82$ & 71.75 \\
$02-03-83$ & 75.01 \\
$09-01-83$ & 76.65
\end{tabular}


Table 4.-- Water levels in selected wells in Fort Bend County, 1969-87--Cont inued

Well JY-65-34-604---Cont.

$\begin{array}{ll}02-16-84 & 74.38 \\ 01-21-85 & 77.39 \\ 09-18-85 & 79.52 \\ 01-16-86 & 64.18 \\ 02-18-87 & 63.06\end{array}$

Well JY-65-34-701

Owner: City of Needville Screen: $307-417$ feet

Al ti tude: 93 feet

\begin{tabular}{ll}
\hline & Water \\
Date & level \\
\hline
\end{tabular}

$01-18-72 \quad 63.66$

$01-19-73 \quad 65.82$

$01-16-74 \quad 63.37$

$01-17-75 \quad 63.72$

$01-14-76 \quad 68.79$

$01-17-77 \quad 68.80$

$03-21-78 \quad 64.71$

02-22-79 $\quad 67.45$

08-02-79 $\quad 76.60$

01-26-81 $\quad 69.82$

08-13-81 79.42

02-24-82 75.65

07-23-82 $\quad 81.74$

03-15-83 $\quad 69.53$

09-01-83 84.13

02-22-84 $\quad 71.07$

10-01-85 80.06

01-16-86 $\quad 69.75$

02-18-87 $\quad 71.31$

Well JY-65-34-710

Owner: City of Needville Screen: $306-420$ feet

Altitude: 91 feet

\begin{tabular}{ll}
\hline Date & $\begin{array}{l}\text { Water } \\
\text { level }\end{array}$ \\
\hline $01-16-86$ & 64.46 \\
$02-18-87$ & 65.07
\end{tabular}

Well JY-65-34-901

Owner: Walter Gless

Screen: 84-635 feet

Al titude: 73 feet

\begin{tabular}{cl}
\hline Date & $\begin{array}{l}\text { Water } \\
\text { level }\end{array}$ \\
\hline $01-23-69$ & 49 \\
$01-29-70$ & 50.20 \\
$01-11-71$ & 49.21 \\
$01-17-72$ & 50.36 \\
$01-18-73$ & 50.18 \\
$01-16-74$ & 43.31 \\
$01-20-75$ & 45.18 \\
$01-14-76$ & 44.82 \\
$01-17-77$ & 42.98 \\
$03-20-78$ & 42.93 \\
$02-22-79$ & 44.05 \\
$01-26-81$ & 49.29 \\
$08-11-81$ & 51.50 \\
$03-01-82$ & 45.30 \\
$07-22-82$ & 65.53 \\
$02-03-83$ & 43.89 \\
$09-01-83$ & 60.05 \\
$01-17-77$ & 25.59 \\
$08-03-77$ & 26.97 \\
$02-28-79$ & 28.09
\end{tabular}

Well JY-65-34-901---Cont.

$\begin{array}{ll}02-16-84 & 58.23 \\ 01-21-85 & 39.21 \\ 09-18-85 & 42.52 \\ 01-16-86 & 39.56\end{array}$

Well JY-65-35-101

Owner: Gulf Oil Corp.

Depth: 86 feet

Altitude: 81 feet

\begin{tabular}{cc}
\hline Date & Water \\
\hline $01-23-69$ & 27.75 \\
$08-12-69$ & 28.63 \\
$01-29-70$ & 27.85 \\
$08-13-70$ & 24.86 \\
$01-12-71$ & 28.07 \\
$08-13-71$ & 23.13 \\
$01-18-72$ & 25.57 \\
$08-07-72$ & 29.14 \\
$01-19-73$ & 28.77 \\
$08-13-73$ & 25.47 \\
$01-17-74$ & 28.83 \\
$08-07-74$ & 29.64 \\
$01-15-75$ & 29.78 \\
$08-08-75$ & 30.45 \\
$01-14-76$ & 29.24 \\
$08-04-76$ & 30.89 \\
$01-17-77$ & 30.65 \\
$08-03-77$ & 31.75 \\
$03-21-78$ & 30.64 \\
$08-09-78$ & 31.60 \\
$02-28-79$ & 30.95 \\
$08-03-79$ & 32.24 \\
$01-17-80$ & 30.61 \\
$08-07-80$ & 32.75 \\
$01-26-81$ & 31.51 \\
$08-11-81$ & 33.31 \\
$02-23-82$ & 26.89 \\
$07-22-82$ & 28.26 \\
$02-03-83$ & 25.71 \\
$08-11-83$ & 28.80 \\
$02-16-84$ & 27.27 \\
$01-21-85$ & 27.06 \\
$10-01-85$ & 28.46 \\
$01-27-86$ & 27.10 \\
& \\
&
\end{tabular}

Well JY-65-35-102

Owner: Gulf oil Corp.

Depth: 180 feet

Altitude: 81 feet

\begin{tabular}{cc}
\hline Date & $\begin{array}{l}\text { Water } \\
\text { level }\end{array}$ \\
\hline $01-23-69$ & 26.11 \\
$08-12-69$ & 26.49 \\
$01-29-70$ & 26.74 \\
$08-13-70$ & 27.40 \\
$01-12-71$ & 26.97 \\
$08-13-71$ & 27.16 \\
$01-18-72$ & 27.37 \\
$08-07-72$ & 27.20 \\
$01-19-73$ & 27.97 \\
$08-13-73$ & 27.28 \\
$01-17-74$ & 27.47 \\
$08-07-74$ & 26.49 \\
$01-15-75$ & 26.13 \\
$08-08-75$ & 26.60 \\
$01-14-76$ & 26.99 \\
$08-03-79$ & 28.12 \\
$01-17-80$ & 27.70 \\
$08-07-80$ & 28.32
\end{tabular}

Well JY-65-35-102--Cont.

$\begin{array}{ll}01-26-81 & 28.20 \\ 08-11-81 & 28.55 \\ 07-22-82 & 27.69 \\ 02-03-83 & 28.42 \\ 08-11-83 & 28.74 \\ 02-16-84 & 27.03 \\ 01-27-86 & 28.90 \\ 02-18-86 & 30.13\end{array}$

Well JY-65-35-201

Owner: A.P. George, Est. Screen: $735-803$ feet

Altitude: 75 feet

\begin{tabular}{ll}
\hline Date & $\begin{array}{l}\text { Well } \\
\text { level }\end{array}$ \\
\hline $01-27-86$ & 85.80
\end{tabular}

Well JY-65-35-302

Owner: Houston Lighting and Power Co.. W. A. Parrish, Well no. 1

Screen: $540-690$ feet Altitude: 74 feet

\begin{tabular}{ll}
\hline & Well \\
Date & level \\
\hline
\end{tabular}

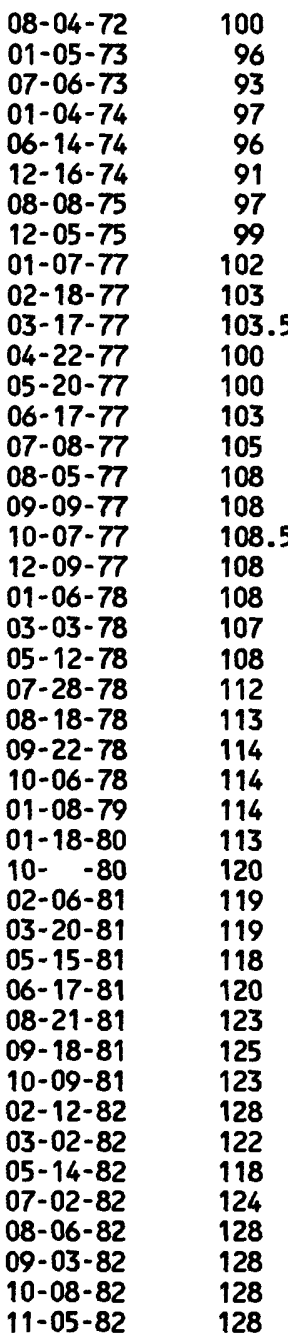


Table 4.-- Water levels in selected wells in Fort Bend County, 1969-87--Cont inued

Well JY-65-35-302--Cont.

$\begin{array}{ll}01-07-83 & 129 \\ 02-11-83 & 126 \\ 03-11-83 & 125 \\ 04-15-83 & 124 \\ 05-27-83 & 123 \\ 06-24-83 & 127 \\ 07-08-83 & 128 \\ 09-09-83 & 128 \\ 10-14-83 & 126 \\ 11-04-83 & 127 \\ 12-21-83 & 128 \\ 02-10-84 & 128 \\ 03-16-84 & 126 \\ 04-06-84 & 125 \\ 05-18-84 & 128 \\ 06-08-84 & 128 \\ 07-13-84 & 132 \\ 09-07-84 & 133 \\ 10-05-84 & 131 \\ 11-09-84 & 129 \\ 12-07-84 & 133 \\ 01-11-85 & 130 \\ 02-08-85 & 132 \\ 03-15-85 & 133 \\ 04-26-85 & 130 \\ 05-17-85 & 127 \\ 06-21-85 & 130 \\ 07-12-85 & 131 \\ 08-16-85 & 131 \\ 09-13-85 & 126 \\ 10-11-85 & 134 \\ 11-08-85 & 133 \\ 12-20-85 & 128 \\ 01-10-86 & 132 \\ 02-21-86 & 128 \\ 04-11-86 & 130 \\ 06-13-86 & 132 \\ 07-11-86 & 128 \\ 08-08-86 & 132 \\ 09-05-86 & 133 \\ 11-14-86 & 129 \\ 12-12-86 & 127 \\ 01-09-87 & 128 \\ 0 & \end{array}$

Well JY-65-35-303

Owner: Houston Lighting and Power Co. W.A. Parrish, Well no. 2

Screen: 457-790 feet

Altitude: 72 feet

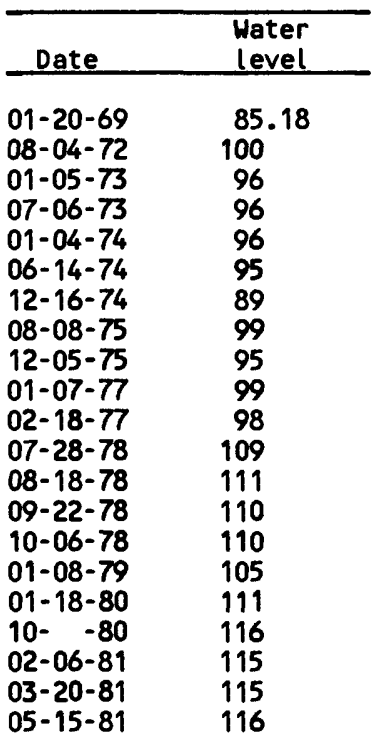

Well JY-65-35-303--Cont.

$\begin{array}{ll}06-26-81 & 120 \\ 08-21-81 & 121 \\ 09-18-81 & 123 \\ 10-09-81 & 121 \\ 02-12-82 & 126 \\ 03-02-82 & 120 \\ 05-14-82 & 117 \\ 07-06-82 & 123 \\ 08-06-82 & 128 \\ 09-03-82 & 129 \\ 10-08-82 & 130 \\ 11-05-82 & 125 \\ 01-07-83 & 125 \\ 02-11-83 & 124 \\ 03-11-83 & 121 \\ 05-27-83 & 123 \\ 06-24-83 & 123 \\ 07-08-83 & 124 \\ 09-09-83 & 123 \\ 10-14-83 & 126 \\ 11-04-83 & 123 \\ 12-21-83 & 123 \\ 02-10-84 & 124 \\ 03-16-84 & 121 \\ 04-06-84 & 123 \\ 05-18-84 & 123 \\ 06-08-84 & 126 \\ 07-13-84 & 129 \\ 09-07-84 & 128 \\ 10-05-84 & 128 \\ 11-09-84 & 125 \\ 12-07-84 & 128 \\ 01-11-85 & 125 \\ 02-08-85 & 128 \\ 03-15-85 & 125 \\ 04-26-85 & 119 \\ 05-17-85 & 126 \\ 06-21-85 & 126 \\ 07-12-85 & 126 \\ 08-16-85 & 129 \\ 09-13-85 & 132 \\ 10-11-85 & 131 \\ 12-20-85 & 126 \\ 01-10-86 & 127 \\ 02-21-86 & 124 \\ 04-11-86 & 123 \\ 06-13-86 & 125 \\ 07-11-86 & 126 \\ 08-08-86 & 126 \\ 09-05-86 & 129 \\ 11-14-86 & 126 \\ 12-12-86 & 124 \\ 01-09-87 & 123 \\ 0 & \end{array}$

Well JY-65-35-304

Owner: Houston Lighting and Power Co.; W. A. Parrish, Well no. 3

Screen: $453-836$ feet

Altitude: 70 feet

\begin{tabular}{lr}
\hline Date & $\begin{array}{r}\text { Water } \\
\text { Level }\end{array}$ \\
\hline $08-04-72$ & 100 \\
$01-05-73$ & 96 \\
$07-06-73$ & 97 \\
$01-04-74$ & 102 \\
$06-14-74$ & 101 \\
$12-16-74$ & 93 \\
$08-08-75$ & 102 \\
$12-05-75$ & 102 \\
$01-07-77$ & 105 \\
$02-18-77$ & 103 \\
$03-17-77$ & 103
\end{tabular}

Well JY-65-35-304--Cont.

\begin{tabular}{|c|}
\hline $\begin{array}{l}04-22-77 \\
05-20-77 \\
06-17-77 \\
07-08-77 \\
08-05-77 \\
09-09-77 \\
10-07-77 \\
11-10-77 \\
01-06-78 \\
03-03-78 \\
05-12-78 \\
07-28-78 \\
08-18-78 \\
09-22-78 \\
10-06-78 \\
01-08-79 \\
10-1-80 \\
02-06-81 \\
03-20-81 \\
05-15-81 \\
06-17-81 \\
08-21-81 \\
09-18-81 \\
10-09-81 \\
02-12-82 \\
03-02-82 \\
05-14-82 \\
07-02-82 \\
08-06-82 \\
09-03-82 \\
10-08-82 \\
11-05-82 \\
01-07-83 \\
02-11-83 \\
03-11-83 \\
04-15-83 \\
05-27-83 \\
06-24-83 \\
07-08-83 \\
09-09-83 \\
10-14-83 \\
11-04-83 \\
12-21-83 \\
02-10-84 \\
03-16-84 \\
04-06-84 \\
05-18-84 \\
06-08-84 \\
07-13-84 \\
09-07-84 \\
10-05-84 \\
11-09-84 \\
12-07-84 \\
01-11-85 \\
02-08-85 \\
03-15-85 \\
04-26-85 \\
05-17-85 \\
06-21-85 \\
07-12-85 \\
08-16-85 \\
09-13-85 \\
10-11-85 \\
11-08-85 \\
12-20-85 \\
01-10-86 \\
02-12-86 \\
04-11-86 \\
06-13-86 \\
07-11-86 \\
08-08-86 \\
09-05-86 \\
11-14-86 \\
12-12-86 \\
01-09-87\end{array}$ \\
\hline
\end{tabular}


Well JY-65-35-701

Owner: Jefferson Lake Sulphur Co.. Well no. 4

Depth: 285 feet

Al titude: 69 feet

\begin{tabular}{cc}
\hline Date & $\begin{array}{l}\text { Hater } \\
\text { level }\end{array}$ \\
\hline $01-29-86$ & 54.11
\end{tabular}

Well JY-65-35-702

Owner: Jefferson Lake Sulphur Co.. Hell no. 5

Screen: $220-725$ feet

Altitude: 67 feet

\begin{tabular}{cc}
\hline Date & $\begin{array}{l}\text { Water } \\
\text { level }\end{array}$ \\
\hline $01-30-86$ & 25.70
\end{tabular}

Well JY-65-35-704

Owner: Jefferson Lake Sulphur Co., Well no. 3

Screen: 247-262 feet

Altitude: 67 feet

\begin{tabular}{cc}
\hline Date & $\begin{array}{l}\text { Water } \\
\text { level }\end{array}$ \\
\hline $01-30-86$ & 54.40
\end{tabular}

Well JY-65-35-707

Owner: Jefferson Lake Sulphur Co.. Well no. 7

Screen: 235-486 feet

Altitude: 67 feet

\begin{tabular}{cc}
\hline Date & $\begin{array}{l}\text { Nater } \\
\text { level }\end{array}$ \\
\hline $01-29-86$ & 64.50 \\
$02-26-87$ & 62.42
\end{tabular}

Well JY-65-35-709

Owner: Jefferson Lake Sulphur Co.. Well no. 9

Screen: $344-494$ feet

Alt itude: 70 feet

\begin{tabular}{ll}
\hline Date & $\begin{array}{l}\text { Water } \\
\text { level }\end{array}$ \\
\hline
\end{tabular}

$01-30-86 \quad 83.88$

Well JY-65-35-710

Owner: Jefferson Lake Sulphur Co. Well no. 10

Screen: 240-503 feet

Altitude: 68 feet

\begin{tabular}{ll}
\hline Date & Water \\
level
\end{tabular}

01-30-86 69.15
Well JY-65-35-711

Owner: Jefferson Lake Sulphur Co.. Well no. 6

Screen: $407-497$ feet

Altitude: 68 feet

\begin{tabular}{cc}
\hline Date & $\begin{array}{l}\text { Nater } \\
\text { level }\end{array}$ \\
\hline $01-29-86$ & 82.19 \\
$02-26-87$ & 80.58
\end{tabular}

Well JY-65-36-201

Owner: chicago Corp.

Depth: 375 feet

Altitude: 58 feet

\begin{tabular}{cc}
\hline Date & $\begin{array}{l}\text { Water } \\
\text { level }\end{array}$ \\
\hline $02-13-87$ & 76.75
\end{tabular}

Well JY-65-36-207

Owner: Chicago Corp.

Depth: 400 feet

Altitude: 58 feet

\begin{tabular}{cc}
\hline Date & $\begin{array}{l}\text { Water } \\
\text { level }\end{array}$ \\
\hline $02-13-87$ & 87.07
\end{tabular}

Well JY-65-36-508

Owner: $W$. P. Sweringen

Depth: 380 feet

Altitude: 56 feet

\begin{tabular}{cc}
\hline Date & $\begin{array}{l}\text { Mater } \\
\text { level }\end{array}$ \\
\hline $02-12-87$ & 56.94
\end{tabular}

Well JY-65-42-10

Owner: L. Krobot

Screen: 110-261 feet

Altitude: 78 feet

\begin{tabular}{cc}
\hline Date & $\begin{array}{l}\text { Water } \\
\text { level }\end{array}$ \\
\hline $01-09-86$ & 40.65 \\
$01-29-87$ & 41.59
\end{tabular}

Hell JY-65-42-105

Owner: H. L. Gray Depth: 1,200 feet

Alt itude: 72 feet

\begin{tabular}{ll}
\hline Date & $\begin{array}{l}\text { Water } \\
\text { level }\end{array}$ \\
\hline $01-09-86$ & 56.71 \\
$01-29-87$ & 67.15
\end{tabular}

Well JY-65-42-206

Owner: Jack Wendt

Screen: 184-1,082 feet

Altitude: 81 feet

\begin{tabular}{ll}
\hline Date & $\begin{array}{l}\text { Water } \\
\text { level }\end{array}$ \\
\hline $01-09-86$ & 87.12 \\
$01-29-87$ & 86.20
\end{tabular}

Hell JY-65-42-207

Owner: J. Moore

Depth: 1,105 feet

Altitude: 82 feet

\begin{tabular}{cc}
\hline Date & $\begin{array}{l}\text { Water } \\
\text { level }\end{array}$ \\
\hline $01-09-86$ & 83.90 \\
$01-29-87$ & 84.20
\end{tabular}

Well JY-65-42-301

Owner: C. A. Danklefs, Hell no. 1

Depth: 545 feet

Altitude: 77 feet

\begin{tabular}{cc}
\hline Date & $\begin{array}{l}\text { Water } \\
\text { level }\end{array}$ \\
\hline $01-09-86$ & 22.56 \\
$01-29-87$ & 20.46
\end{tabular}

Hell JY-65-42-302

Owner: C. A. Danklefs

Screen: 64-551 feet

Altitude: 55 feet

\begin{tabular}{cc}
\hline Date & $\begin{array}{l}\text { Hater } \\
\text { level }\end{array}$ \\
\hline $01-10-86$ & 24.98
\end{tabular}

Well JY-65-42-304

Owner: A. Bosak

Depth: 110 feet

Altitude: 76 feet

\begin{tabular}{cc}
\hline Date & $\begin{array}{l}\text { Water } \\
\text { Level }\end{array}$ \\
\hline $01-10-86$ & 24.37
\end{tabular}

Hell JY-65-43-101

Owner: C. A. Danklefs

Screen: 275-1,195 feet Alt itude: 76 feet

\begin{tabular}{cc}
\hline Date & $\begin{array}{l}\text { Hater } \\
\text { level }\end{array}$ \\
\hline $01-10-69$ & 73.38 \\
$01-29-70$ & 73.64 \\
$01-12-71$ & 81.21 \\
$01-17-72$ & 77.74 \\
$01-23-73$ & 73.64 \\
$01-17-74$ & 74.39 \\
$01-17-75$ & 72.97
\end{tabular}


Well JY-65-43-101---Cont.

$\begin{array}{rr}01-13-76 & 76.13 \\ 01-12-77 & 79.47 \\ 03-17-78 & 77.71 \\ 02-22-79 & 84.78 \\ 08-03-79 & 94.15 \\ 01-17-80 & 82.80 \\ 08-07-80 & 100.22 \\ 01-27-81 & 84.94 \\ 08-13-81 & 97.94 \\ 02-24-82 & 85.02 \\ 07-23-82 & 95.79 \\ 02-03-83 & 97.88 \\ 08-11-83 & 100.30 \\ 02-22-84 & 98.60 \\ 01-22-85 & 78.51 \\ 10-01-85 & 80.66 \\ 01-07-86 & 93.18 \\ 01-29-87 & 87.53\end{array}$

Well JY-65-43-202

Owner: John Mahlmann Depth: 212 feet Altitude: 58 feet

\begin{tabular}{cc}
\hline Date & $\begin{array}{l}\text { Water } \\
\text { level }\end{array}$ \\
\hline $01-07-86$ & 13.27
\end{tabular}

Well JY-65-43-301

Owner: J. Frank Jungman Depth: 1,155 feet Alt itude: 71 feet

\begin{tabular}{ll}
\hline Date & $\begin{array}{l}\text { Water } \\
\text { level }\end{array}$ \\
\hline $01-07-86$ & 105.97
\end{tabular}

Well JY-65-43-502

Owner: Robert Mueck Screen: 108-128 feet Altitude: 54 feet

\begin{tabular}{ll}
\hline Date & $\begin{array}{l}\text { Water } \\
\text { level }\end{array}$ \\
\hline $01-07-86$ & 17.27
\end{tabular}

Well JY-65-43-504 Owner: C. and J. Gless Screen: 277-756 feet Alt itude: 56 feet

\begin{tabular}{ll}
\hline Date & $\begin{array}{l}\text { Water } \\
\text { level }\end{array}$ \\
\hline $01-09-86$ & 92.93 \\
$01-29-87$ & 80.10
\end{tabular}

Well JY-65-43-602

Owner: Unknown

Depth: 482 feet

Altitude: 57 feet

\begin{tabular}{ll}
\hline Date & $\begin{array}{l}\text { Water } \\
\text { level }\end{array}$ \\
\hline $01-23-69$ & 73.10 \\
$08-12-69$ & 74.03 \\
$01-29-70$ & 70.86 \\
$08-14-70$ & 83.02 \\
$01-12-71$ & 69.11 \\
$08-13-71$ & 86.29 \\
$01-17-72$ & 69.07 \\
$08-07-72$ & 85.94 \\
$01-23-73$ & 71.28 \\
$08-10-73$ & 92.46 \\
$01-17-74$ & 72.21 \\
$01-17-75$ & 71.97 \\
$08-08-75$ & 94.10 \\
$01-13-76$ & 73.37 \\
$08-04-76$ & 91.63 \\
$01-17-77$ & 73.20 \\
$08-03-77$ & 96.82 \\
$03-21-78$ & 76.44 \\
$08-09-78$ & 95.75 \\
$02-22-79$ & 80.74 \\
$08-06-79$ & 93.57 \\
$01-17-80$ & 80.15 \\
$08-07-80$ & 97.50 \\
$01-27-81$ & 82.36 \\
$08-13-81$ & 99.02 \\
$03-01-82$ & 84.28 \\
$07-23-82$ & 91.56 \\
$02-03-83$ & 87.05 \\
$08-11-83$ & 88.74 \\
$02-22-84$ & 89.06 \\
$01-22-85$ & 89.70 \\
$10-01-85$ & 90.88 \\
$01-07-86$ & 91.68 \\
& \\
\hline
\end{tabular}

Well JY-65-44-101 Owner: J. Q. Vencil Screen: $216-874$ feet Altitude: 59 feet

\begin{tabular}{ll}
\hline Date & $\begin{array}{l}\text { Water } \\
\text { level }\end{array}$ \\
\hline $01-29-86$ & 94.92 \\
$01-29-87$ & 92.59
\end{tabular}

Well JY-66-24-601

Owner: Valley Lodge

Depth: 227 feet

Altitude: 105 feet

\begin{tabular}{ll|l}
\hline Date & $\begin{array}{l}\text { Water } \\
\text { level }\end{array}$ & \\
\hline $02-24-87$ & 30.85 &
\end{tabular}

Well JY-66-32-902

Owner: Simon Kucera

Depth: 304 feet

Altitude: 113 feet

\begin{tabular}{cc}
\hline Date & $\begin{array}{l}\text { Water } \\
\text { level }\end{array}$ \\
\hline $01-31-86$ & 50.80
\end{tabular}

Well JY-66-32-905

Owner: W. Duncan

Depth: 270 feet

Altitude: 112 feet

\begin{tabular}{cc}
\hline Date & $\begin{array}{l}\text { Water } \\
\text { level }\end{array}$ \\
\hline $01-31-86$ & 47.87 \\
$02-02-87$ & 46.99
\end{tabular}

Well JY-66-32-906

Owner: Bay Ridge Christian College Screen: $75-115$ feet Altitude: 112 feet

\begin{tabular}{ll}
\hline Date & $\begin{array}{l}\text { Water } \\
\text { level }\end{array}$ \\
\hline $01-31-86$ & 35.29
\end{tabular}

Well JY-66-40-304

Owner: Bay Ridge Christian College

Screen: 105-115 feet Altitude: 105 feet

\begin{tabular}{cc}
\hline Date & $\begin{array}{l}\text { Water } \\
\text { level }\end{array}$ \\
\hline $01-13-86$ & 38.32
\end{tabular}

Well JY-66-40-307

Owner: Bay Ridge Christian College

Depth: 324 feet Altitude: 111 feet

\begin{tabular}{cc}
\hline Date & $\begin{array}{l}\text { Water } \\
\text { level }\end{array}$ \\
\hline $01-13-86$ & 46.02 \\
$02-02-87$ & 46.62
\end{tabular}


Table 5.--Average specific-unit compaction values

$$
\text { [ft, foot] }
$$

\begin{tabular}{|c|c|c|c|c|}
\hline site & Period & $\begin{array}{l}\text { Average water- } \\
\text { level decline } \\
(f t)\end{array}$ & $\begin{array}{c}\text { Clay } \\
\text { thickness } \\
(f t)\end{array}$ & $\begin{array}{l}\text { Specific-unit } \\
\text { compaction } \\
\text { (per } f t \text { ) }\end{array}$ \\
\hline Katy & $\begin{array}{l}1906-43 \\
1943-51 \\
1951-54 \\
1954-59 \\
1959-64 \\
1964-73 \\
1973-78 \\
1943-64 \\
1943-73 \\
1943-78 \\
1906-54 \\
1954-78 \\
1906-78\end{array}$ & $\begin{array}{r}62 \\
12 \\
8 \\
9 \\
6 \\
21 \\
9 \\
35 \\
56 \\
65 \\
82 \\
45 \\
127\end{array}$ & $\begin{array}{l}-. \\
\ldots \\
-- \\
-- \\
-. \\
-- \\
-- \\
- \\
-. \\
-. \\
- \\
-- \\
720\end{array}$ & $\begin{array}{c}5.2 \times 10^{-6} \\
2.6 \times 10^{-6} \\
\quad- \\
1.8 \times 10^{-5} \\
1.8 \times 10^{-5} \\
3.3 \times 10^{-5} \\
2.5 \times 10^{-5} \\
8.7 \times 10^{-6} \\
1.7 \times 10^{-5} \\
1.9 \times 10^{-5} \\
4.3 \times 10^{-6} \\
2.7 \times 10^{-5} \\
1.2 \times 10^{-5}\end{array}$ \\
\hline Pasadena $1 /$ & $\begin{array}{l}1906-43 \\
1943-51 \\
1951-54 \\
1954-59 \\
1959-64 \\
1964-73 \\
1973-78 \\
1943-64 \\
1943-73 \\
1943-78 \\
1906-54 \\
1954-78 \\
1906-78\end{array}$ & $\begin{array}{r}177 \\
-- \\
-- \\
-. \\
-- \\
67 \\
5 \\
93 \\
160 \\
166 \\
\ldots \\
\ldots \\
342\end{array}$ & 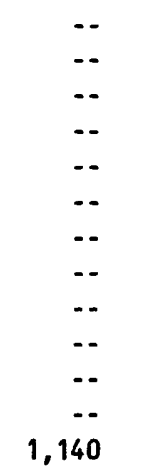 & $\begin{array}{c}4.0 \times 10^{-6} \\
\cdots \\
\cdots \\
-- \\
\cdots \\
4.3 \times 10^{-5} \\
1.6 \times 10^{-5} \\
3.8 \times 10^{-5} \\
4.0 \times 10^{-5} \\
4.3 \times 10^{-5} \\
-- \\
-- \\
2.3 \times 10^{-5}\end{array}$ \\
\hline Addicks 1 & $\begin{array}{l}1906-43 \\
1943-51 \\
1951-54 \\
1954-59 \\
1959-64 \\
1964-73 \\
1973-78 \\
1943-64 \\
1943-73 \\
1943-78 \\
1906-54 \\
1954-78 \\
1906-78\end{array}$ & $\begin{array}{r}68 \\
-- \\
-- \\
-. \\
.- \\
138 \\
34 \\
76 \\
214 \\
248 \\
-. \\
\ldots- \\
316\end{array}$ & $\begin{array}{l}-- \\
-- \\
-- \\
-- \\
-- \\
-. \\
-- \\
-- \\
-- \\
-- \\
-- \\
-. \\
730\end{array}$ & $\begin{array}{c}6.1 \times 10^{-6} \\
\ldots \\
\ldots \\
\ldots \\
- \\
1.0 \times 10^{-5} \\
2.0 \times 10^{-5} \\
1.4 \times 10^{-5} \\
1.2 \times 10^{-5} \\
1.3 \times 10^{-5} \\
\quad- \\
\cdots \\
1.1 \times 10^{-5}\end{array}$ \\
\hline
\end{tabular}

1/ From Gabrysch, 1982, table 1. 


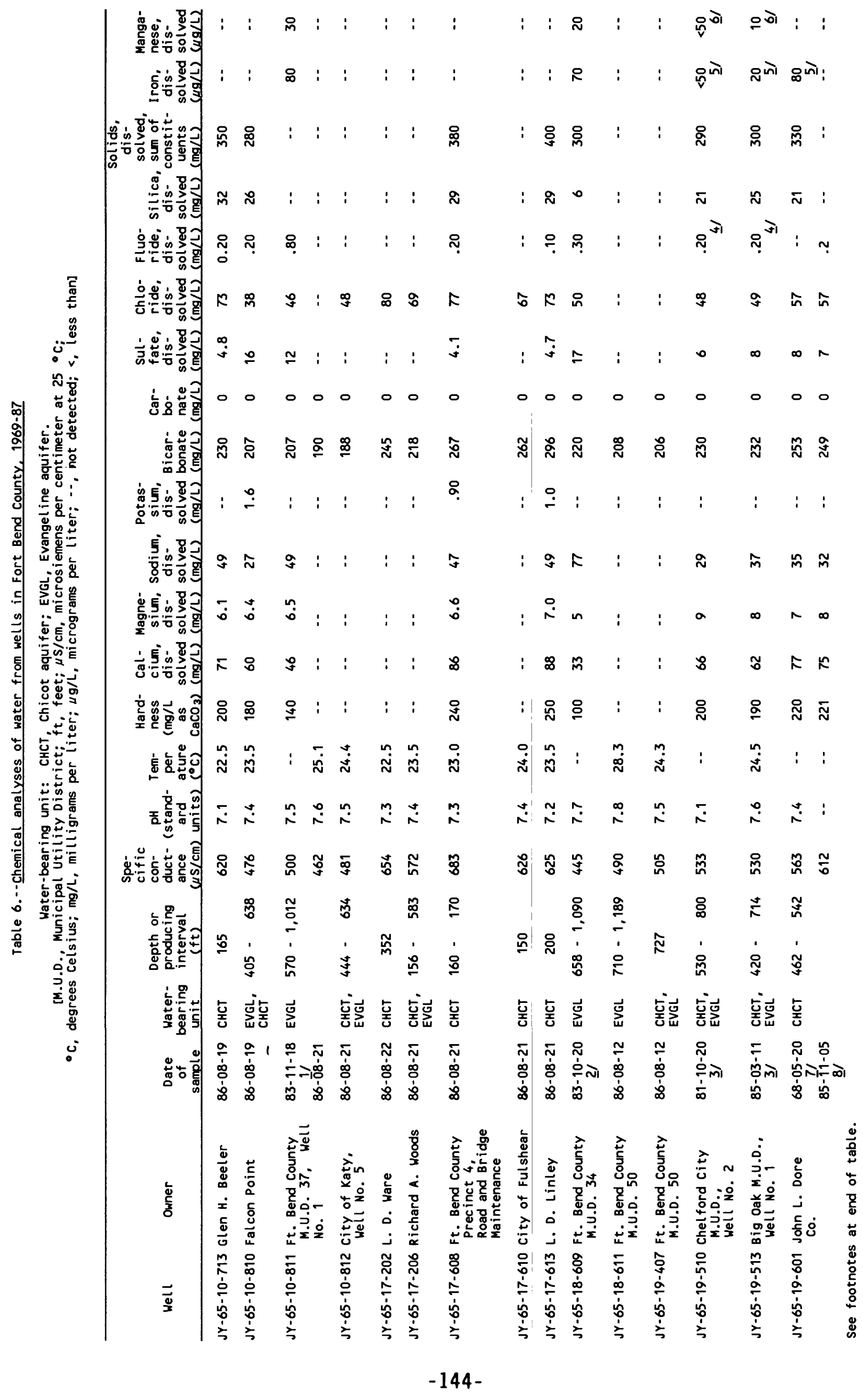




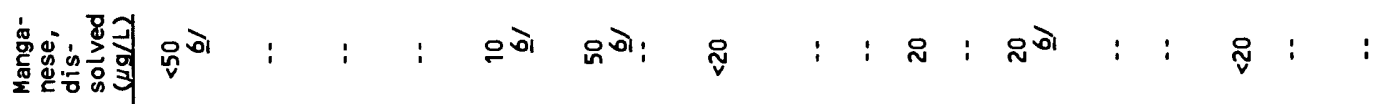

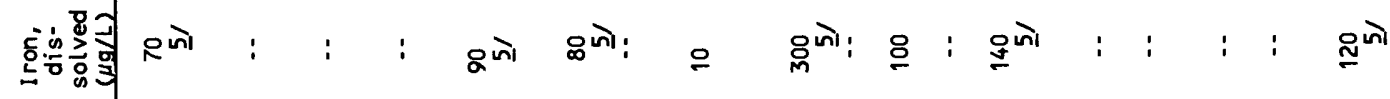

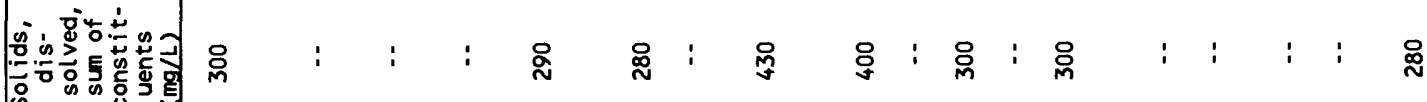

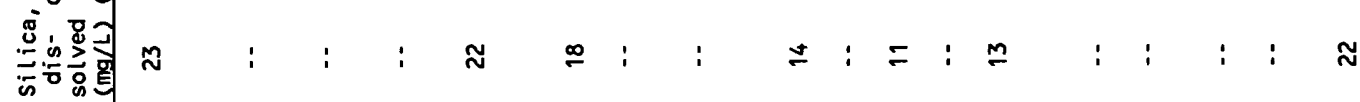

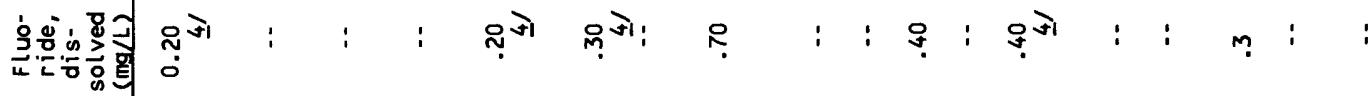

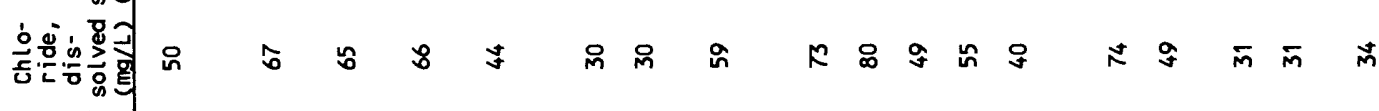

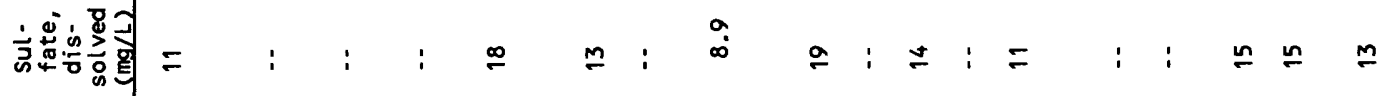

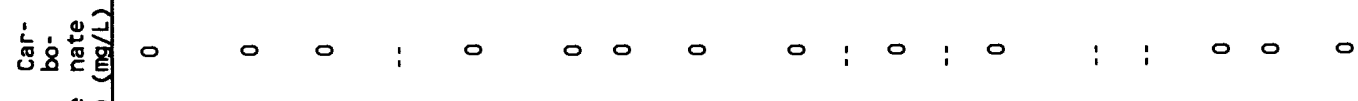

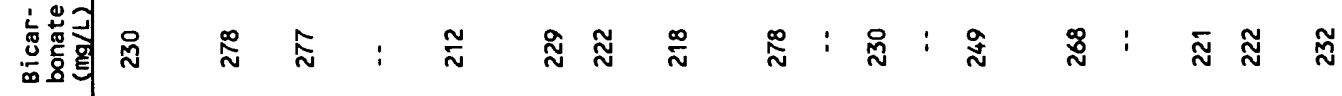

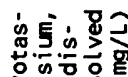

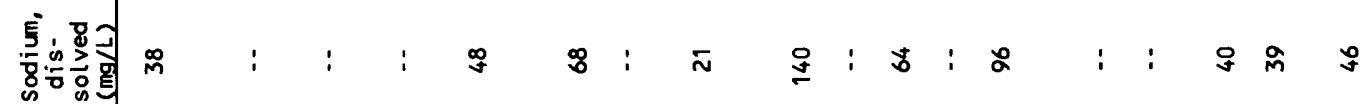

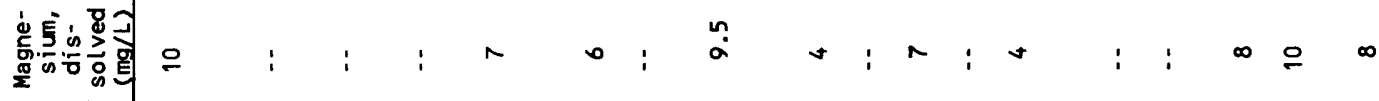

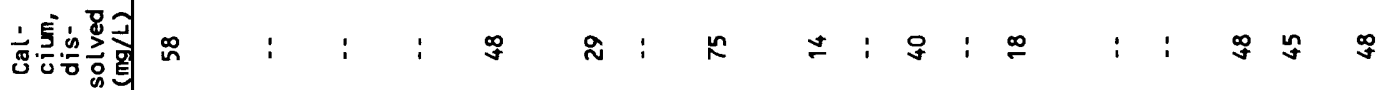

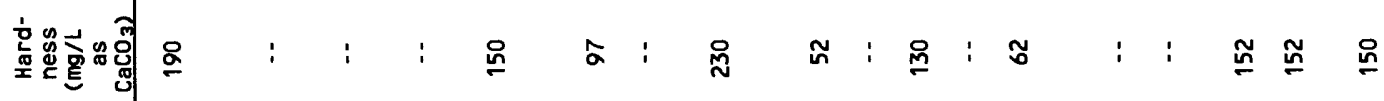

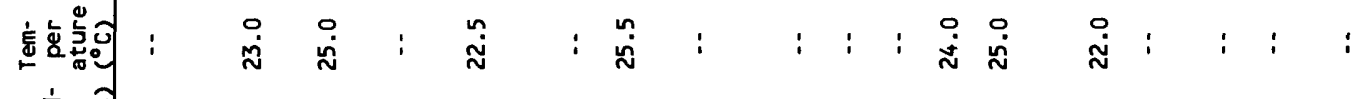

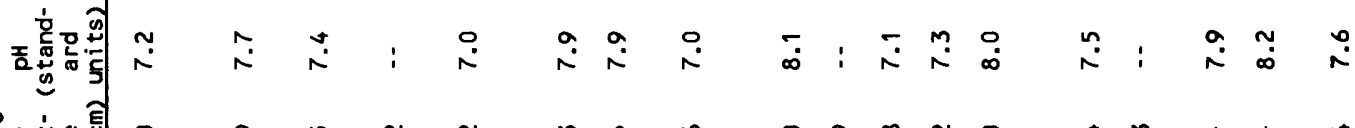

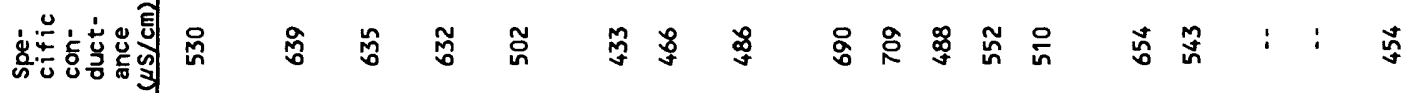

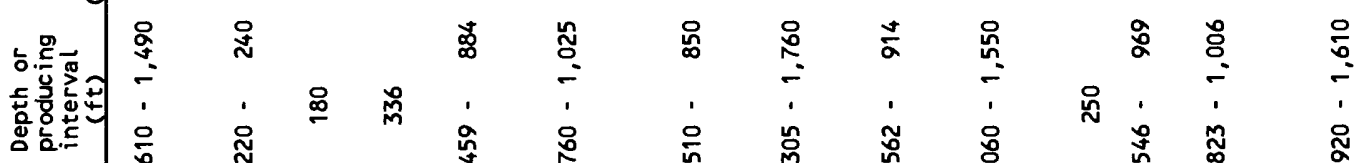

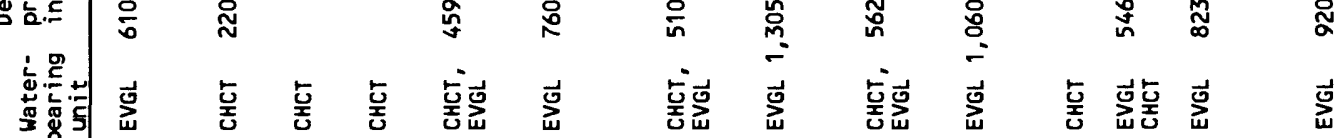

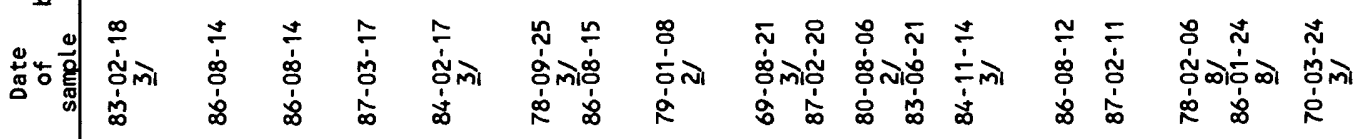

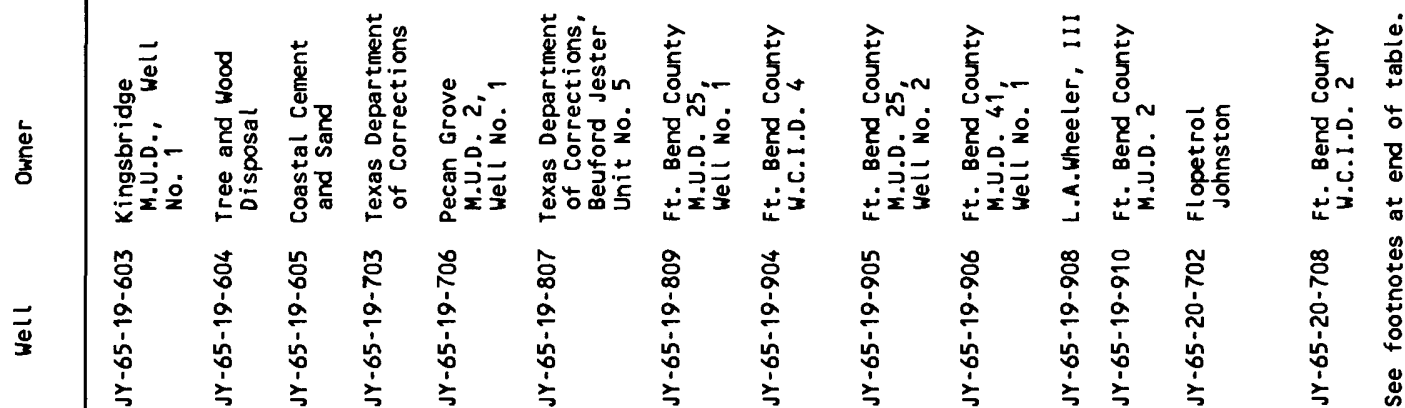




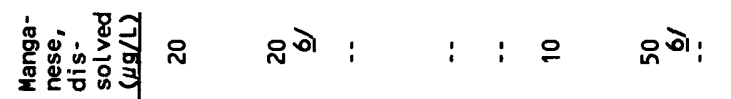

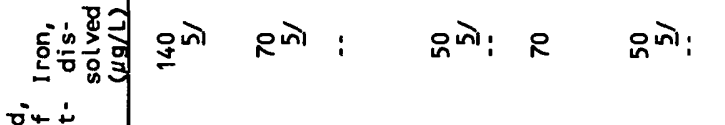

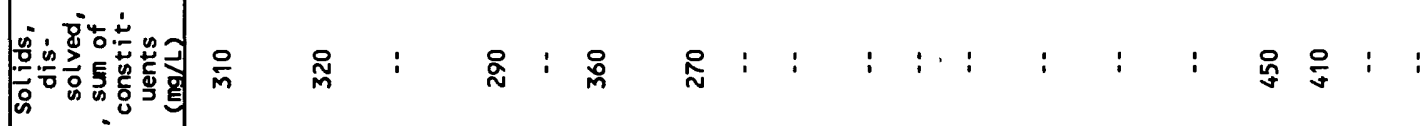

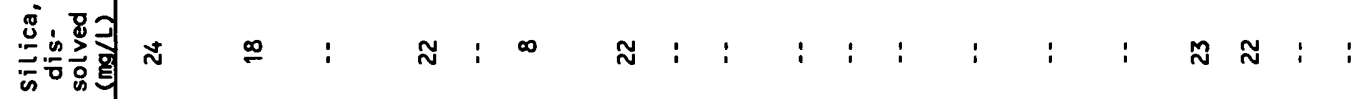

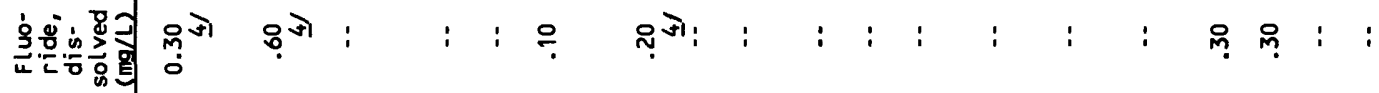

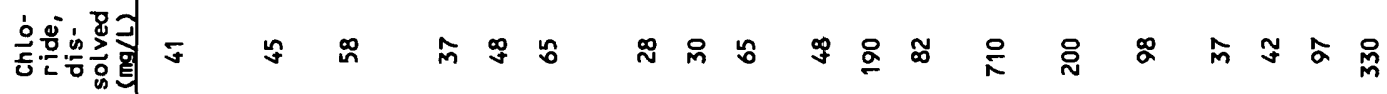

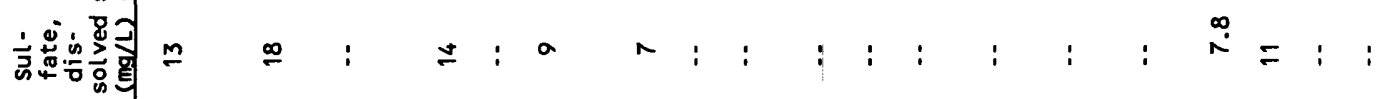

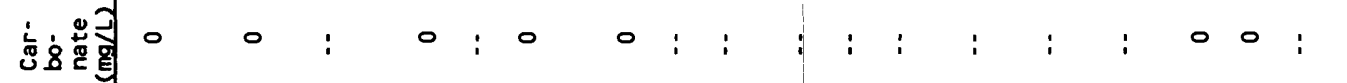

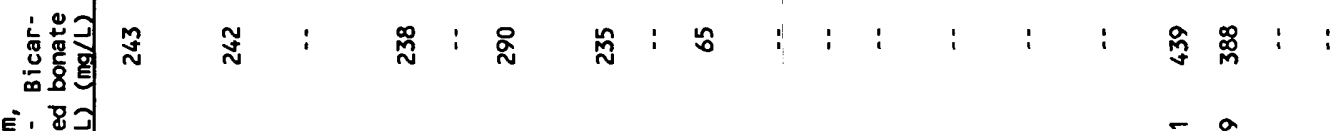

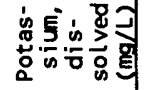

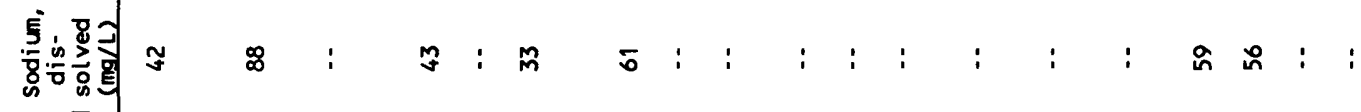

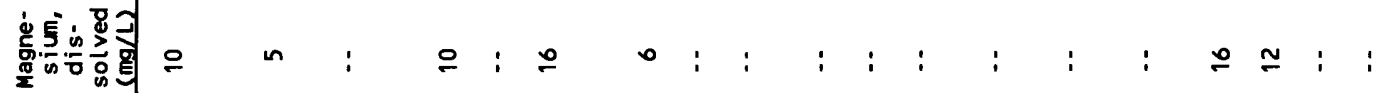

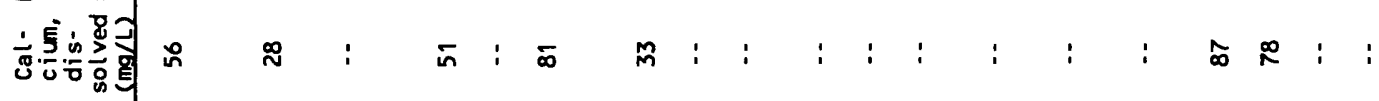

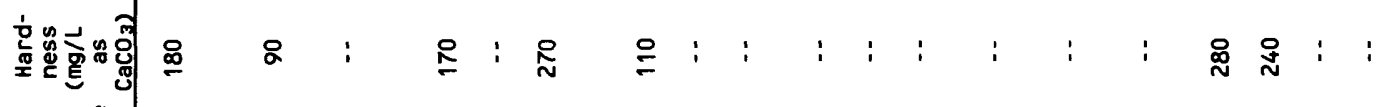

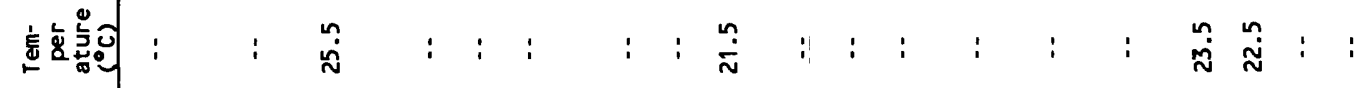

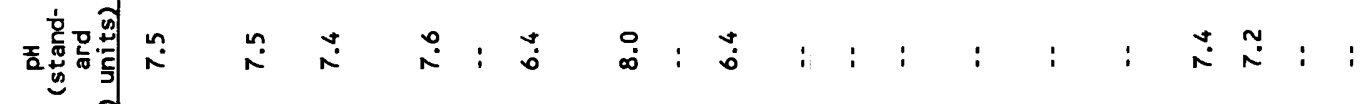

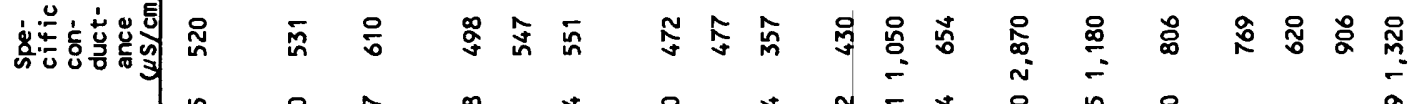

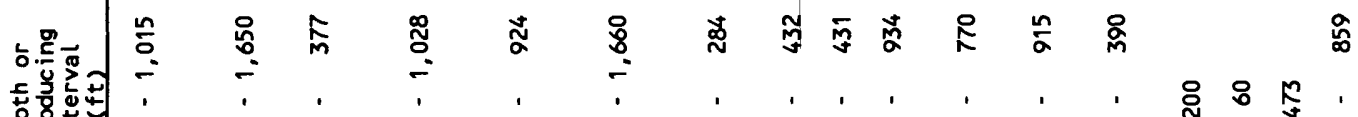

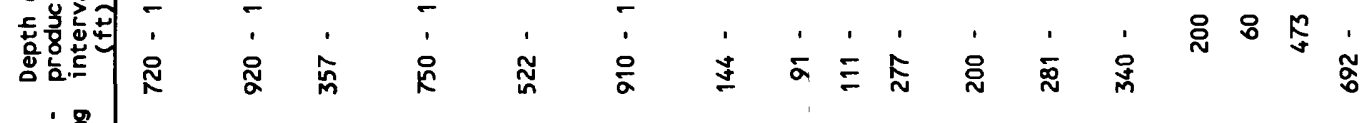

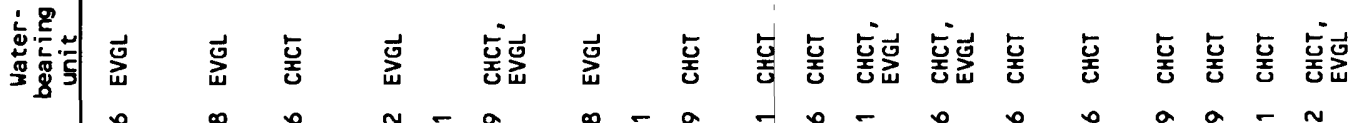

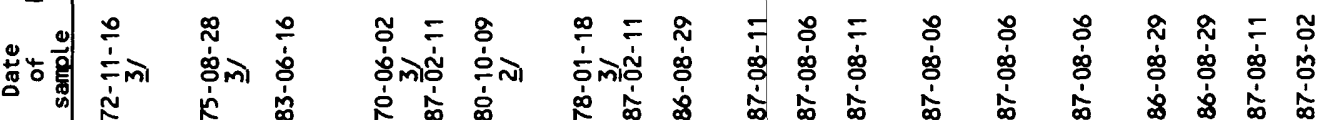

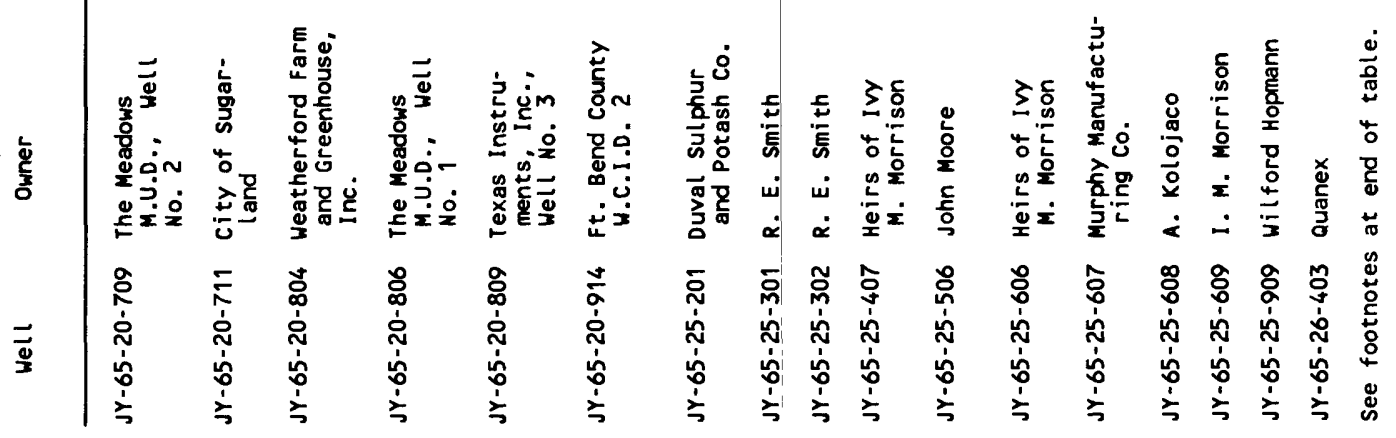




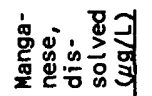

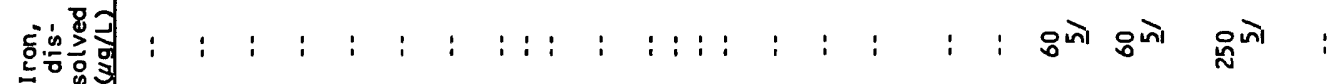

g.

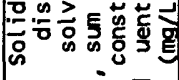

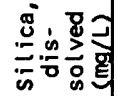

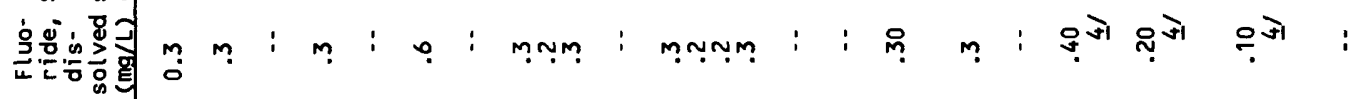

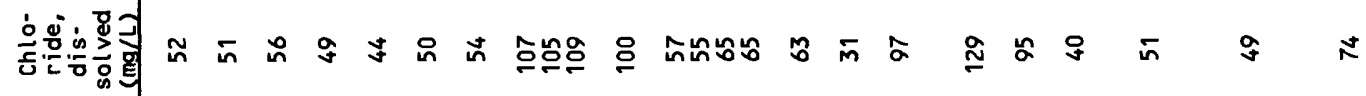

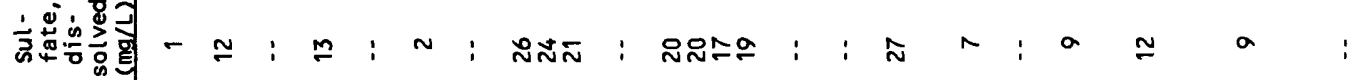

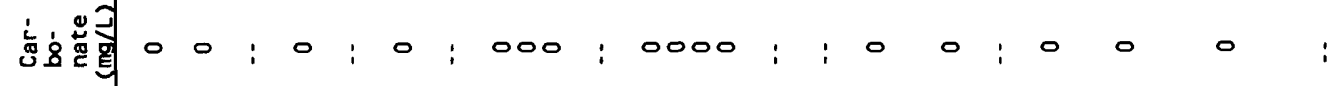

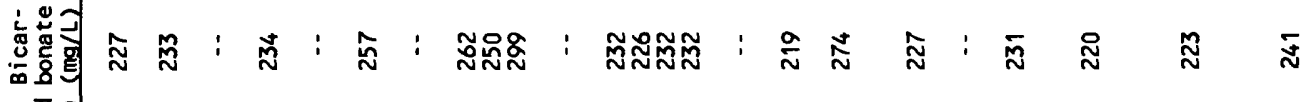
is

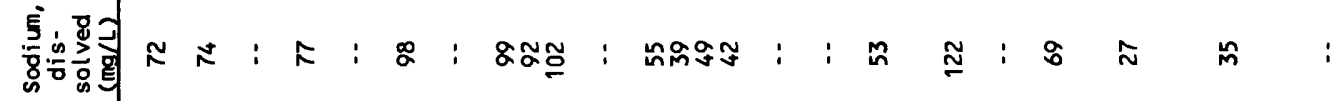

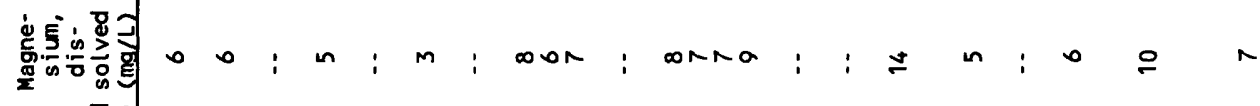

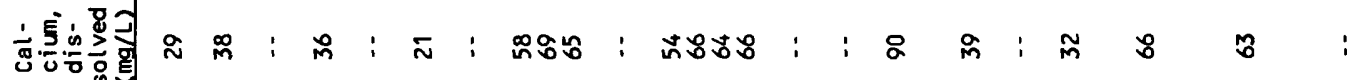

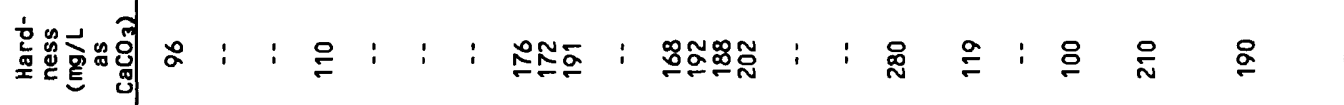

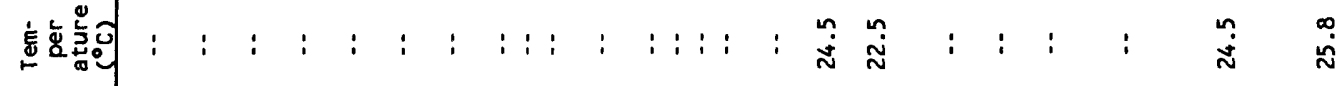

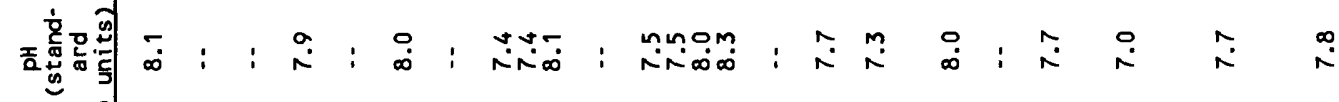

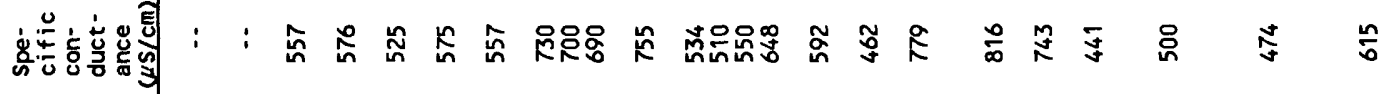

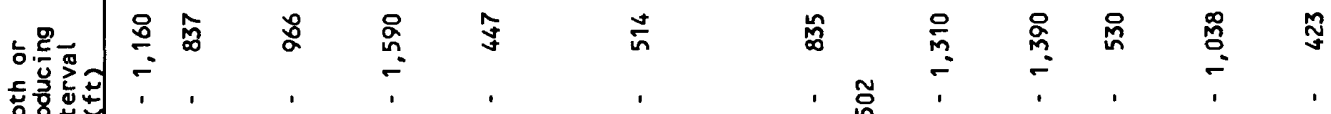

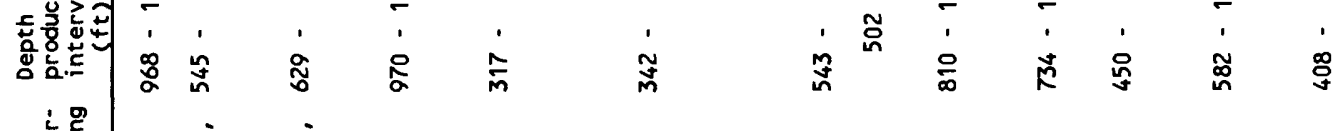

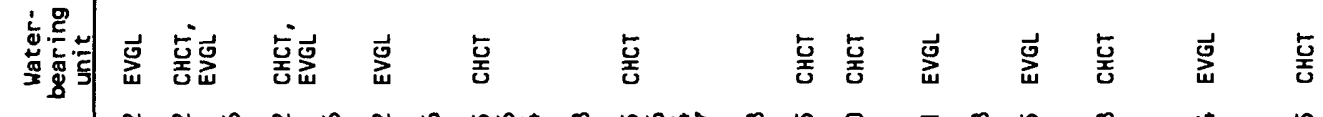
ม⿻

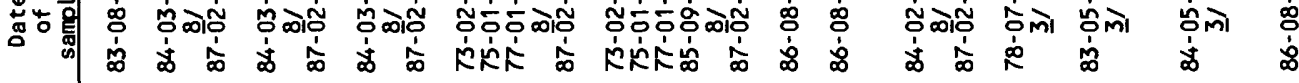

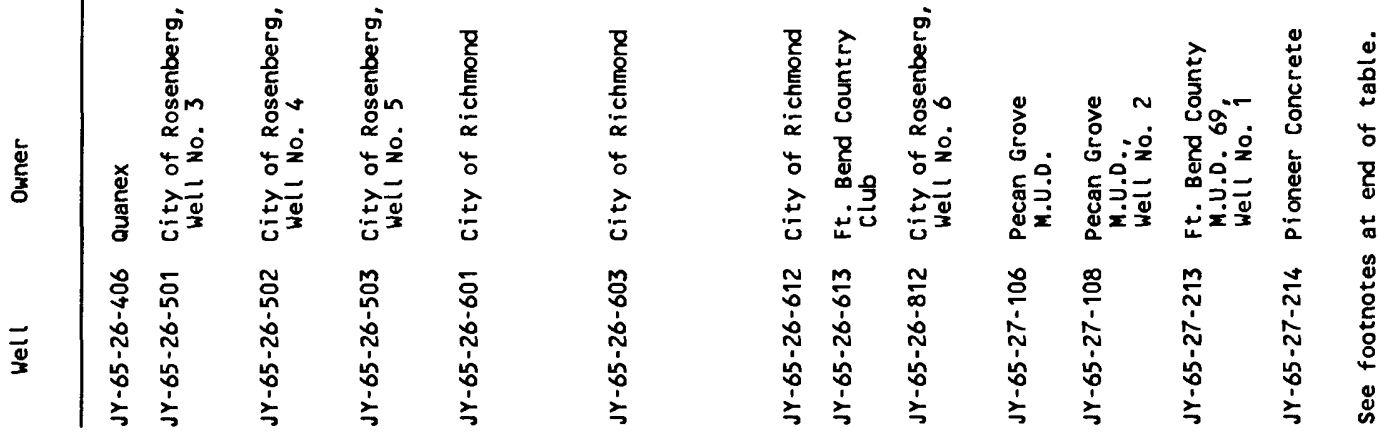




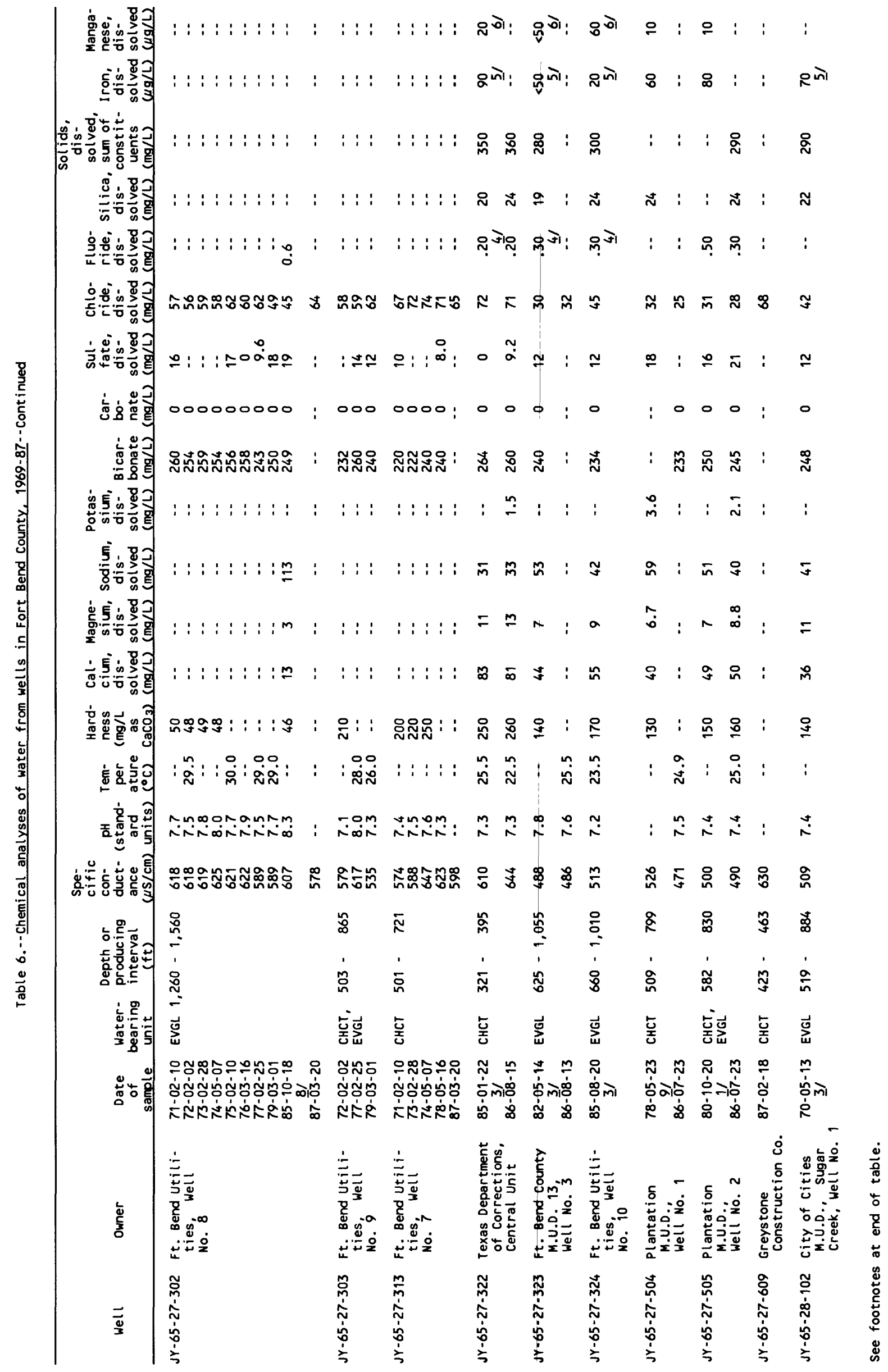




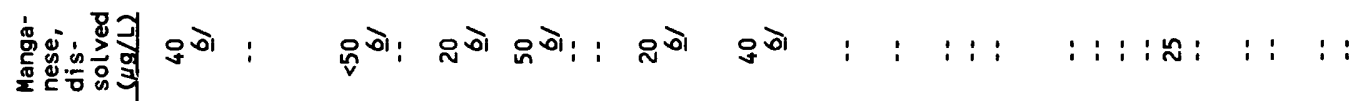

है.

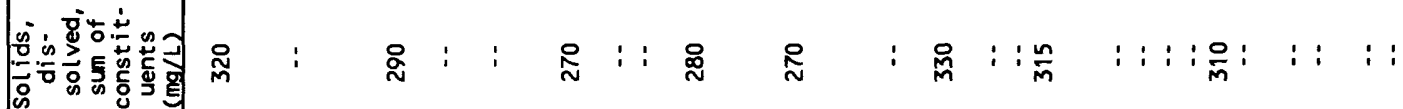

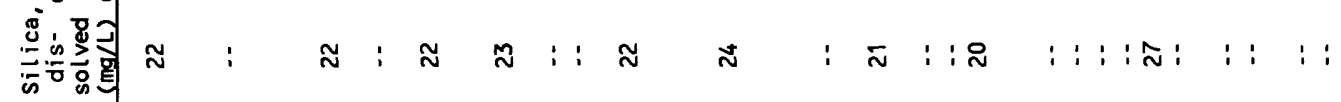

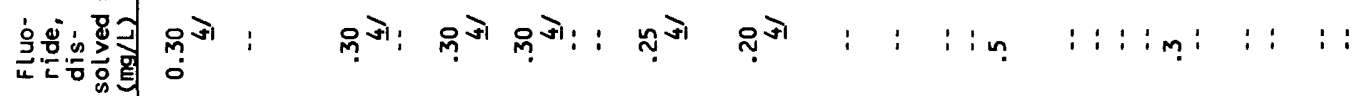

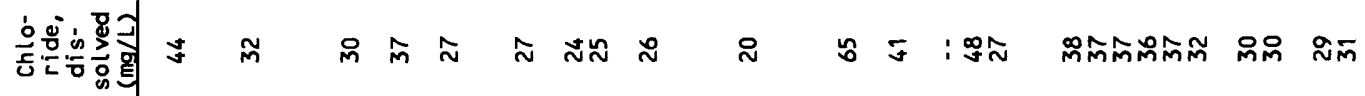

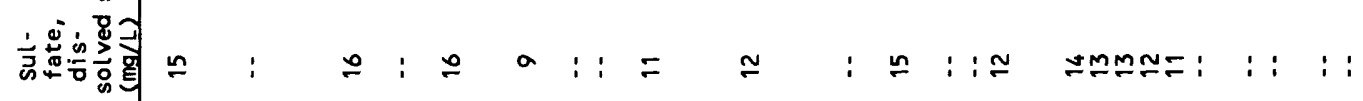

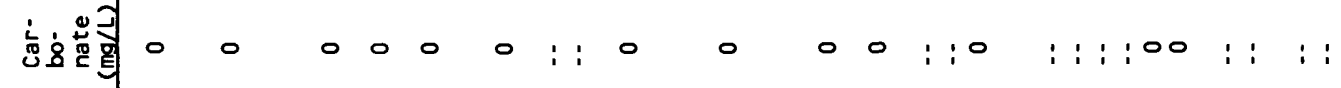

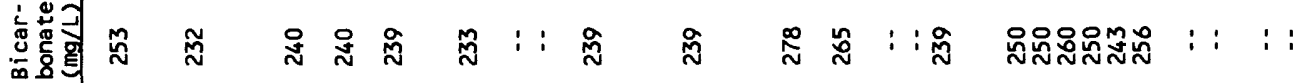

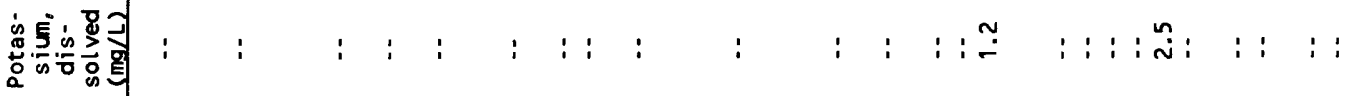

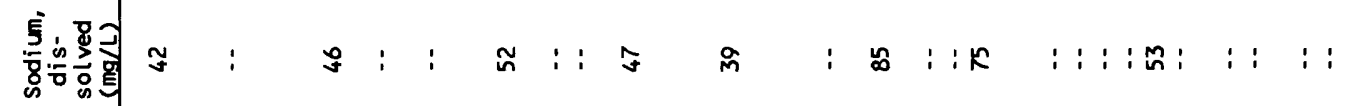

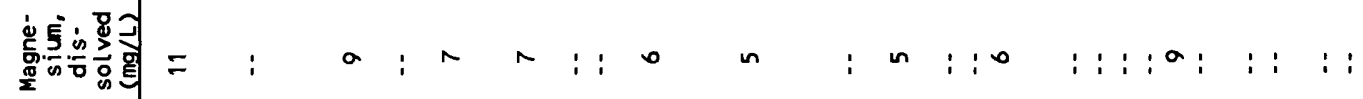

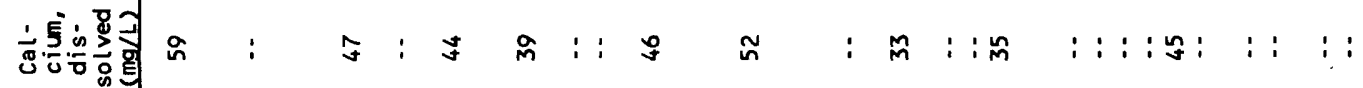

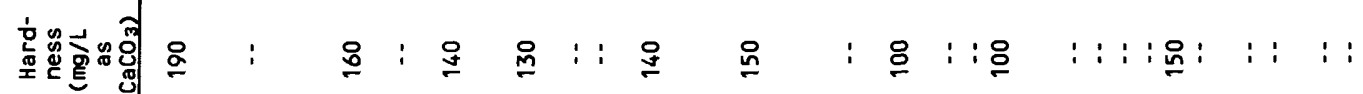

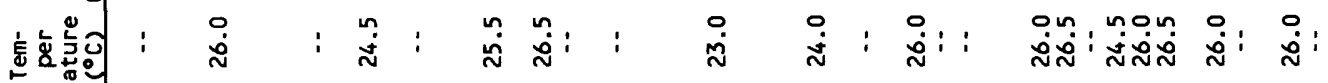

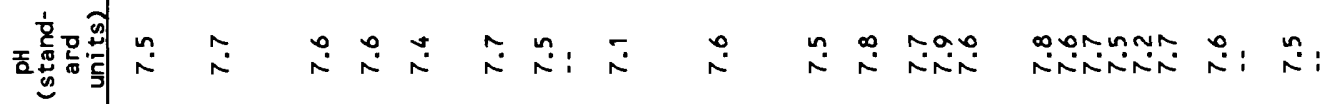

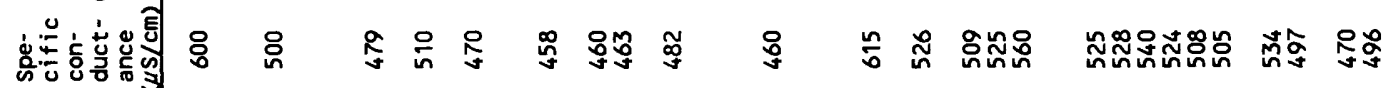

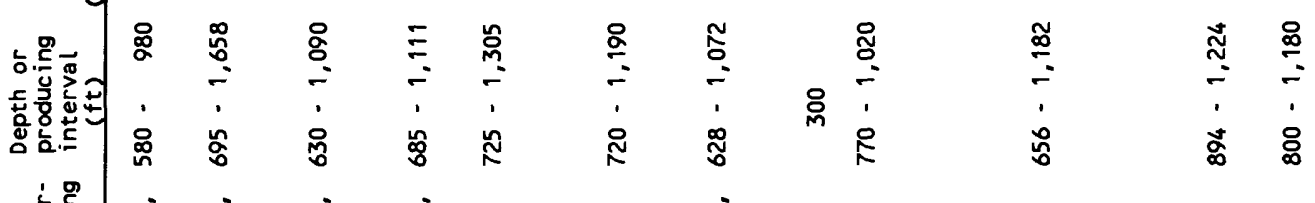

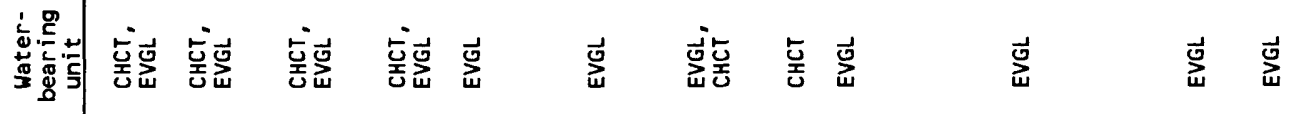

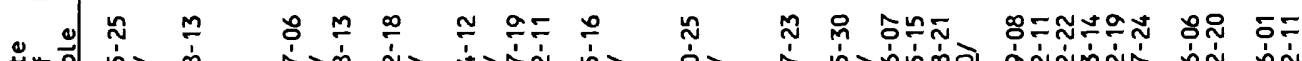

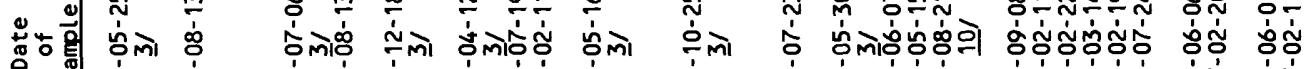

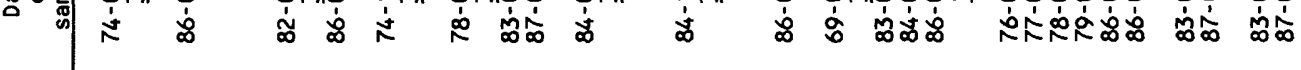

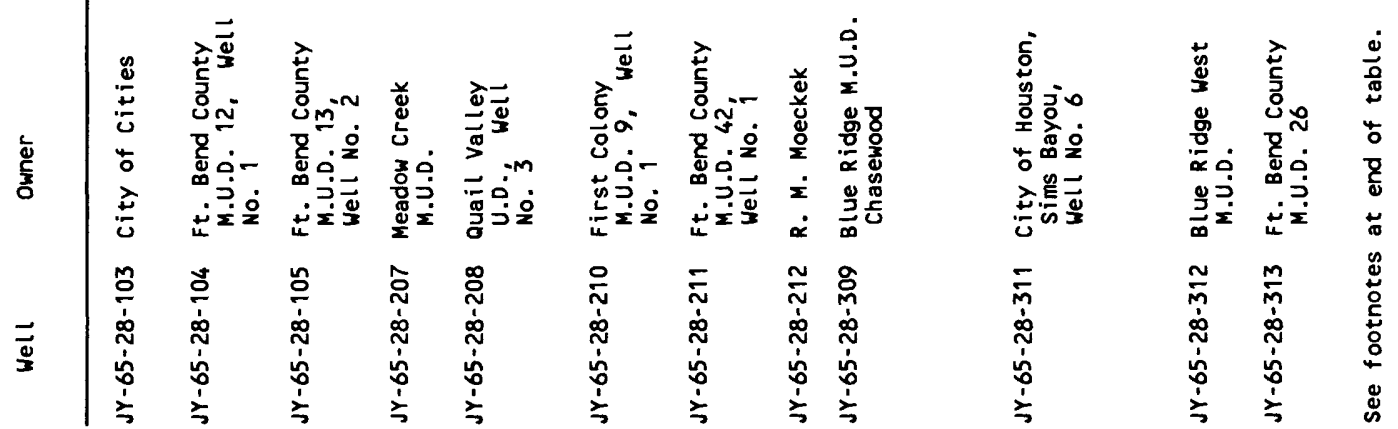




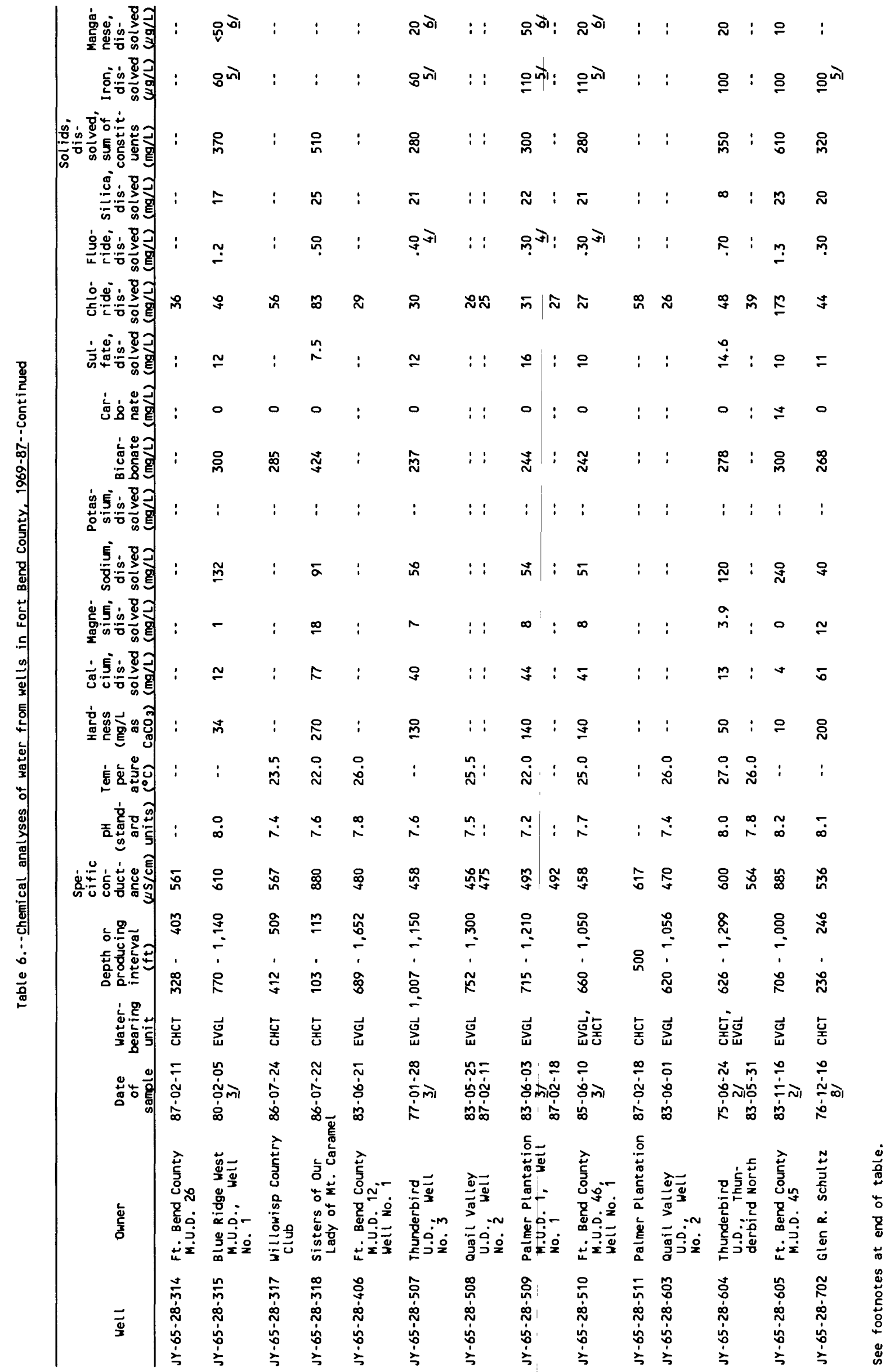




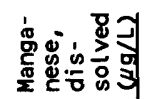

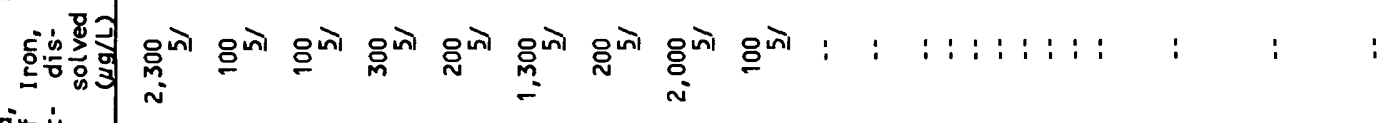

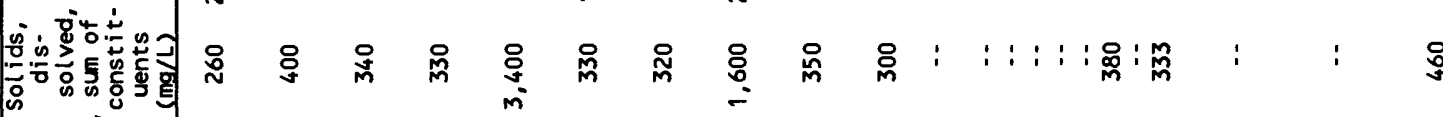

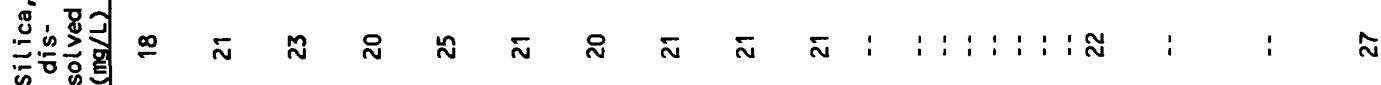

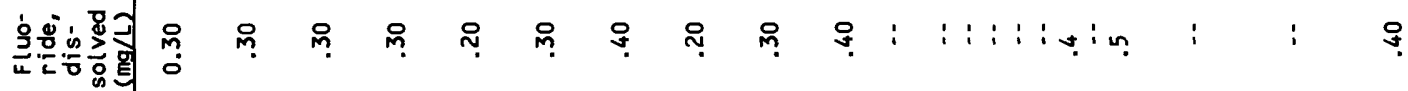

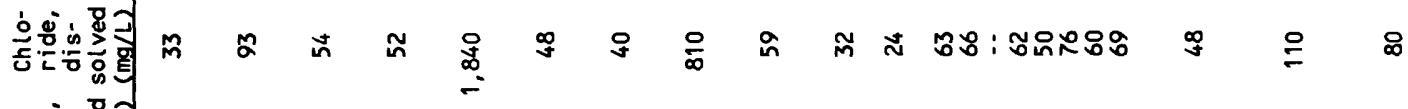

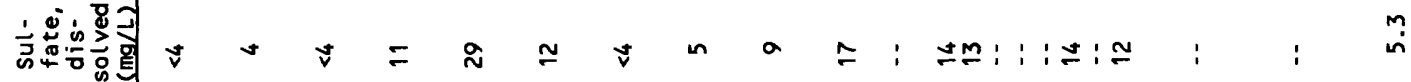

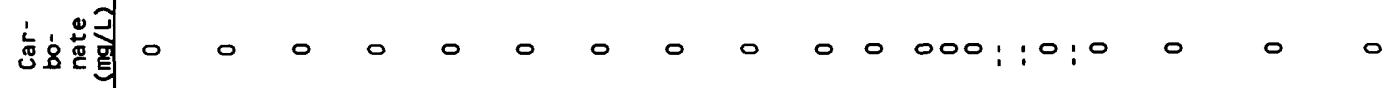

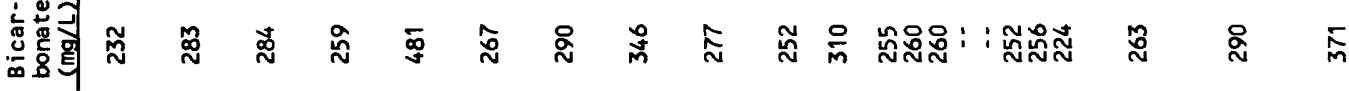

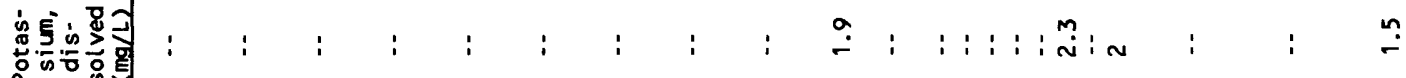

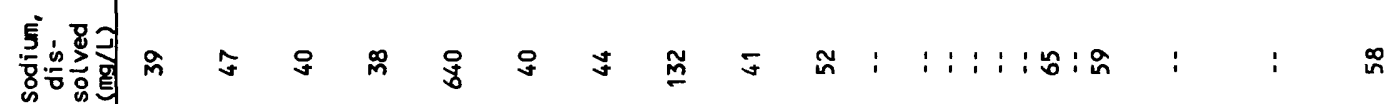

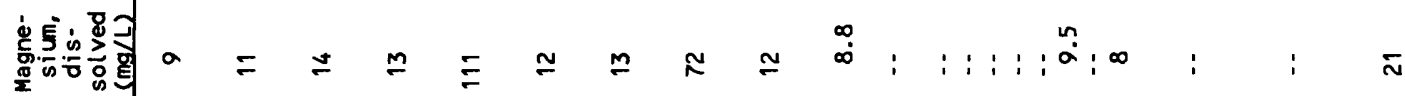

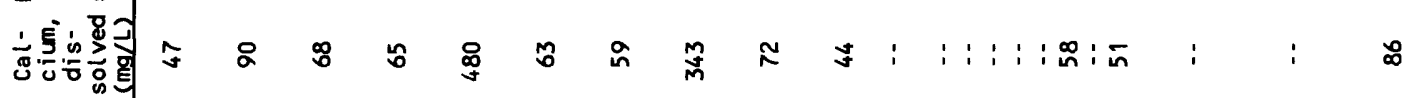

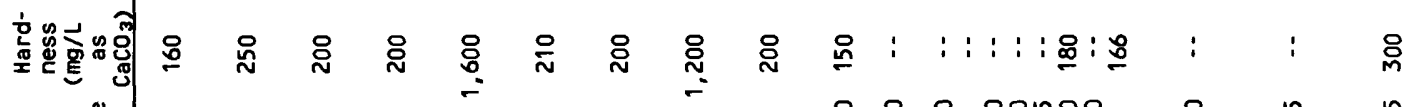

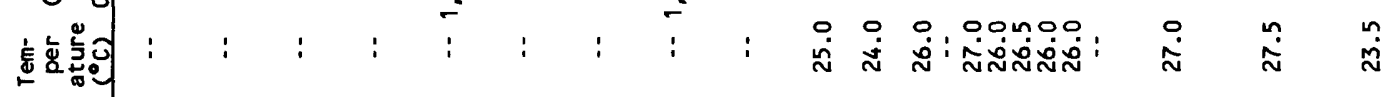

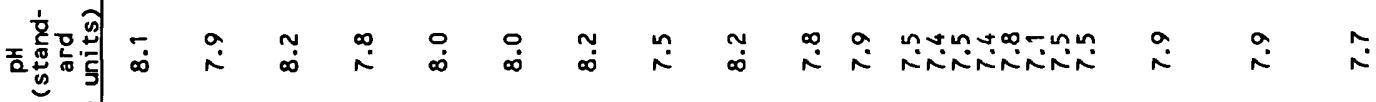

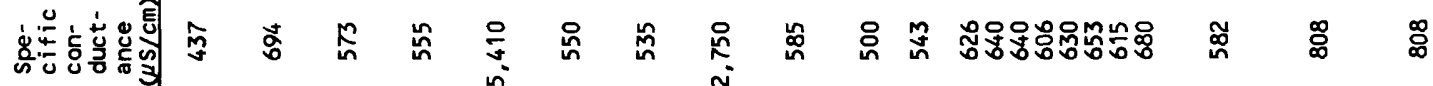

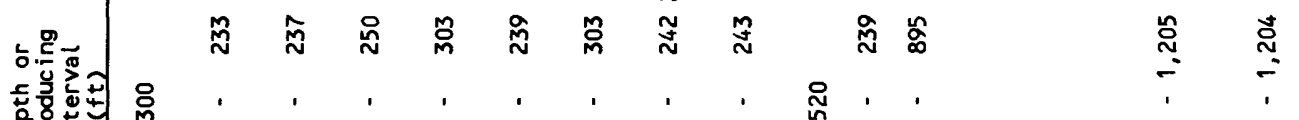

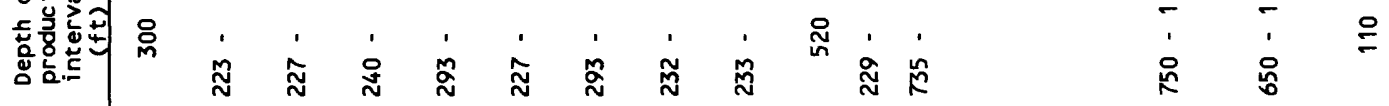

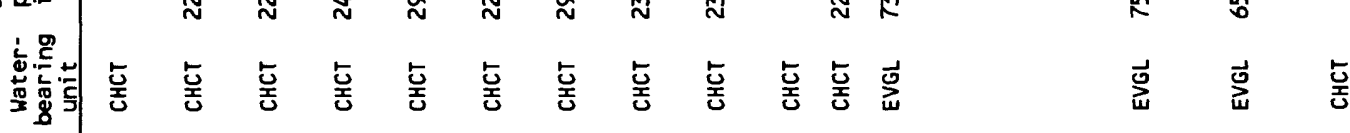

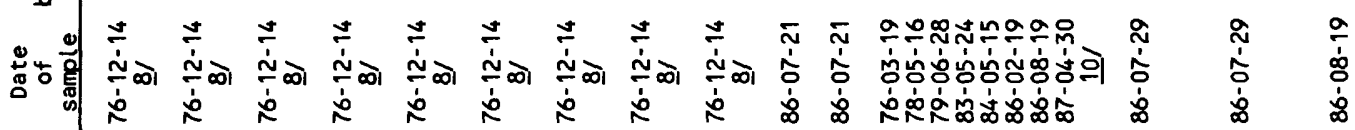

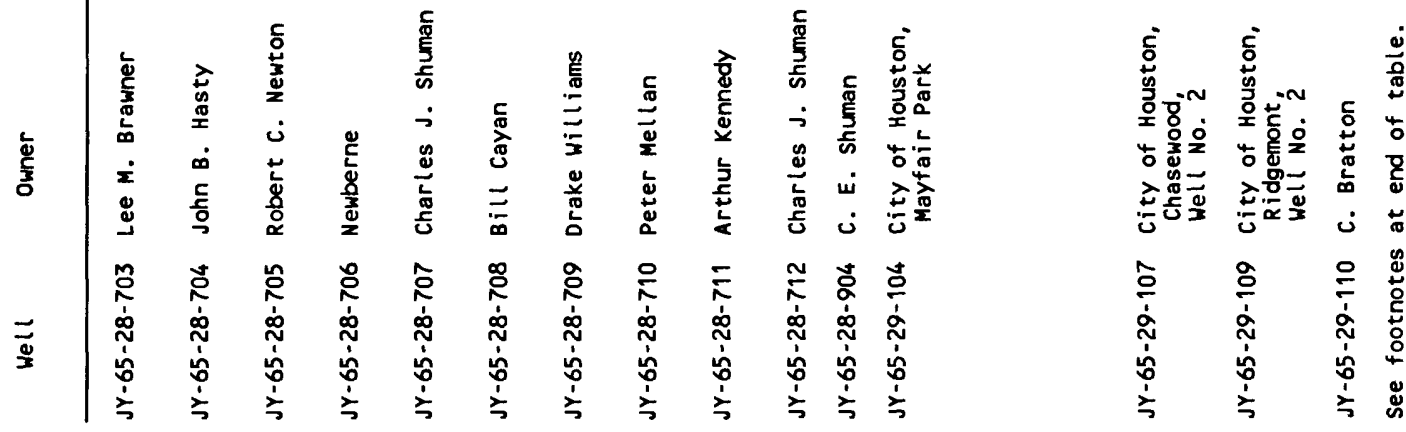




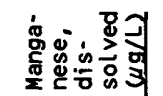

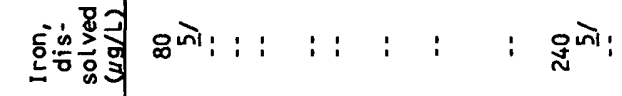

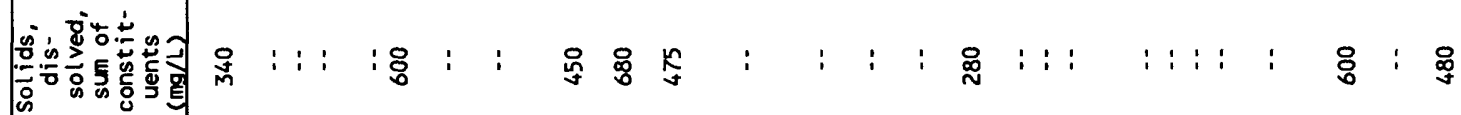

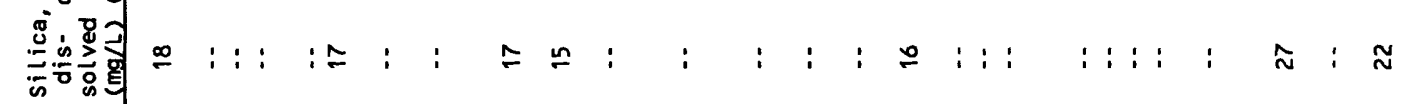

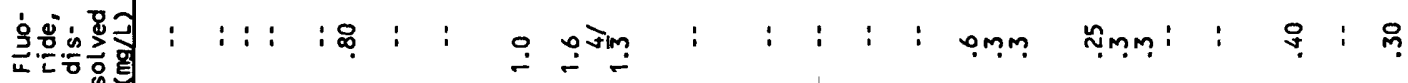

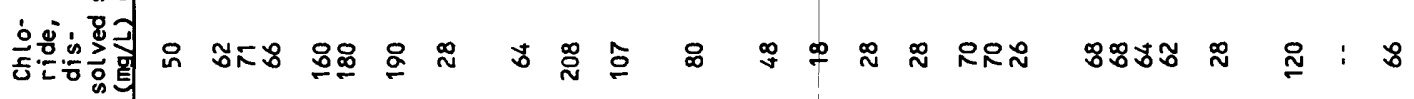

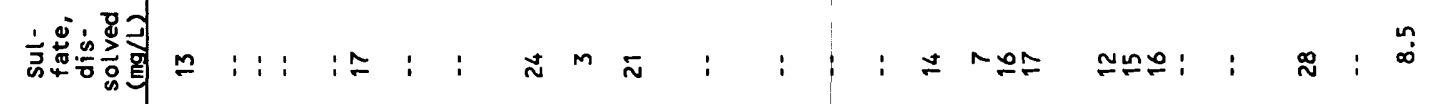

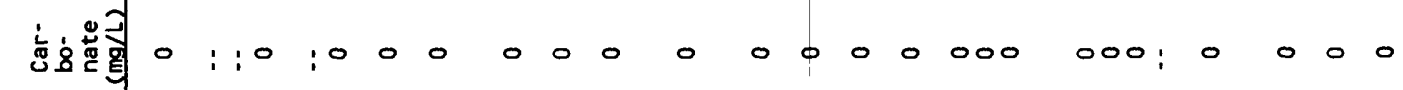

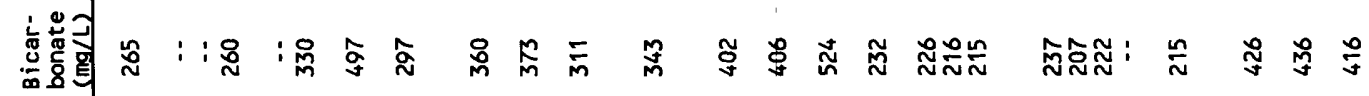

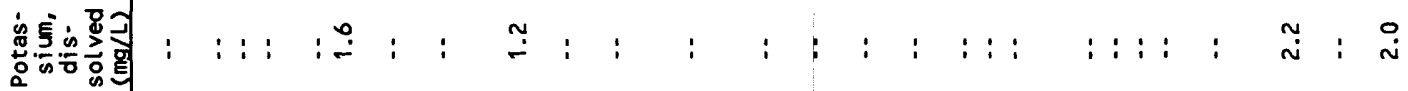

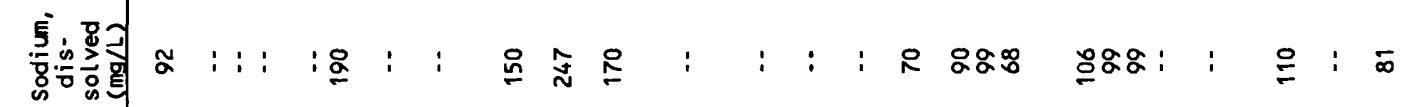

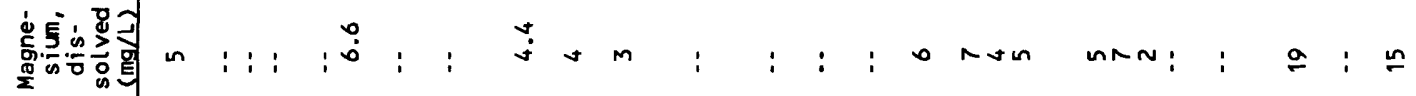

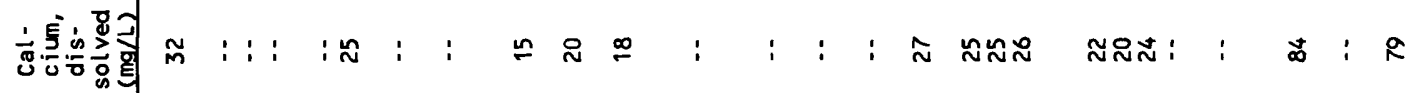

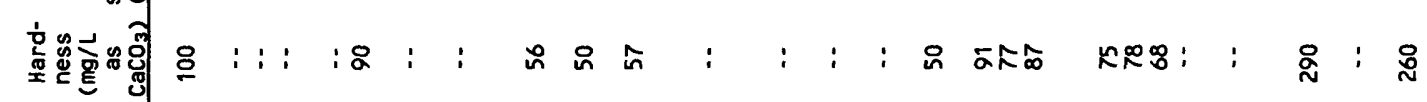

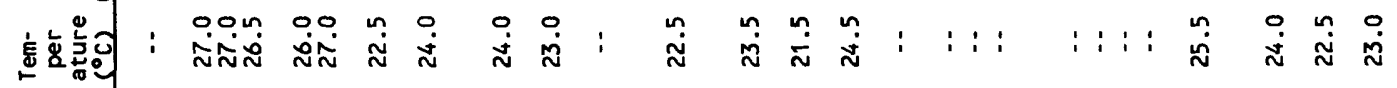

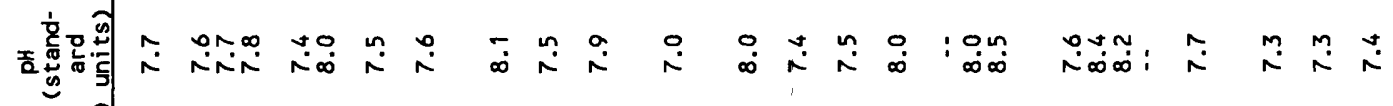

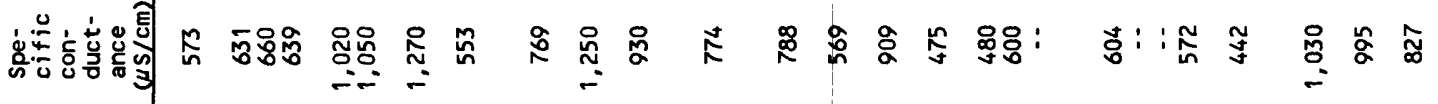

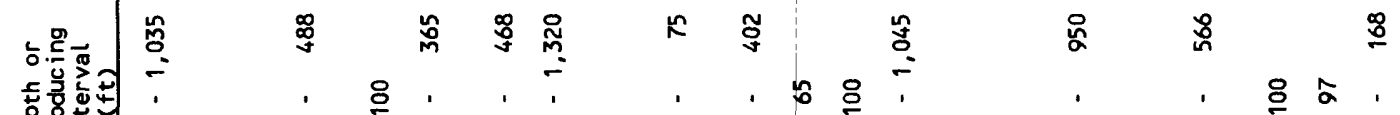

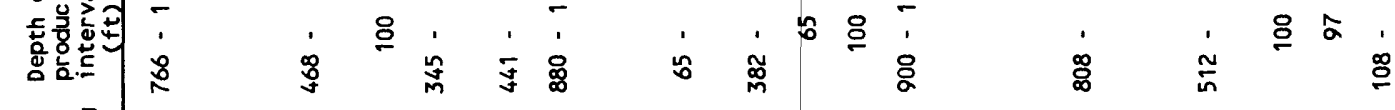

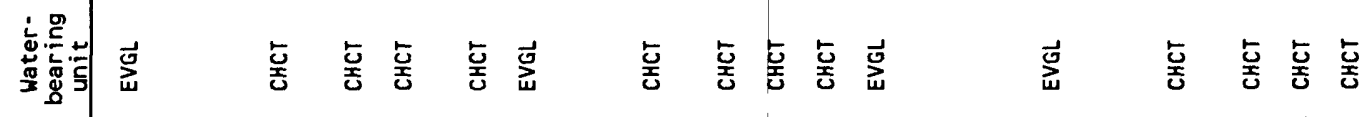

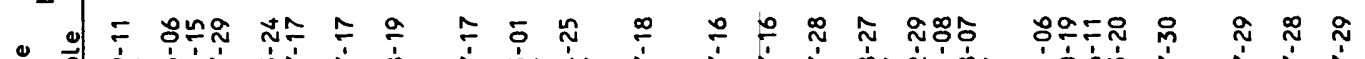

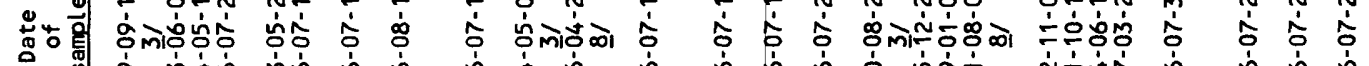

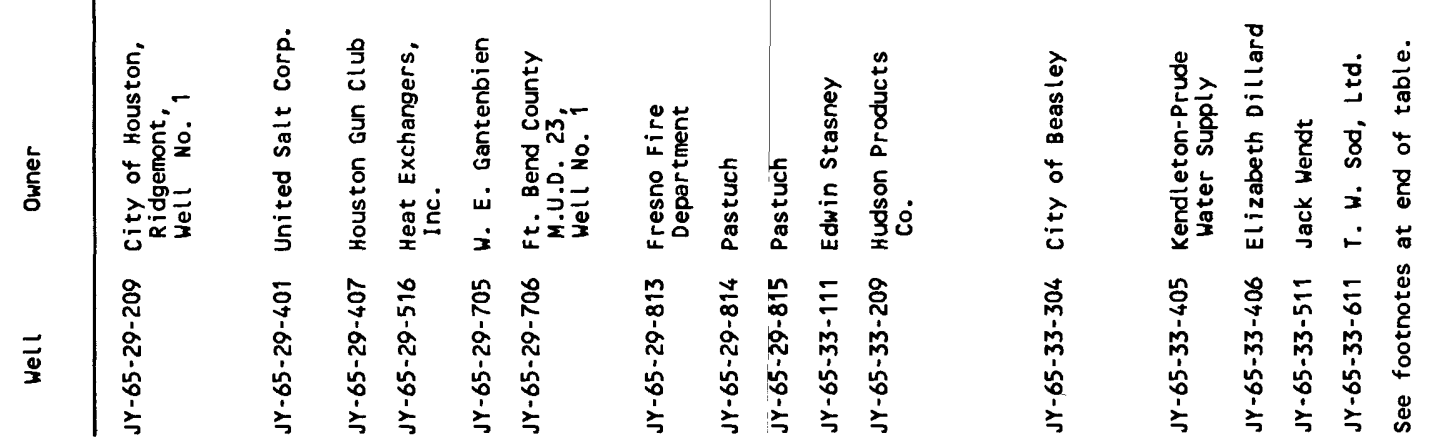




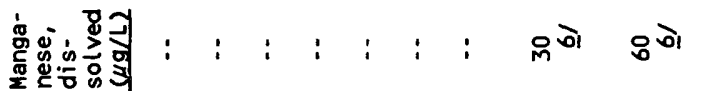

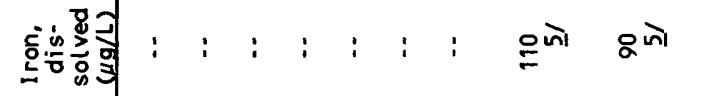

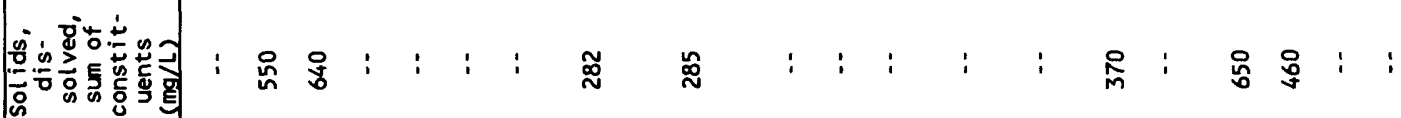

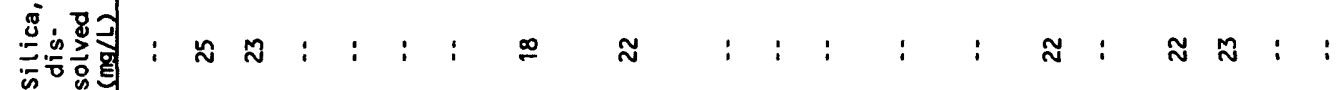

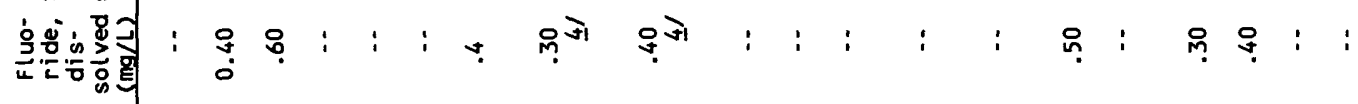

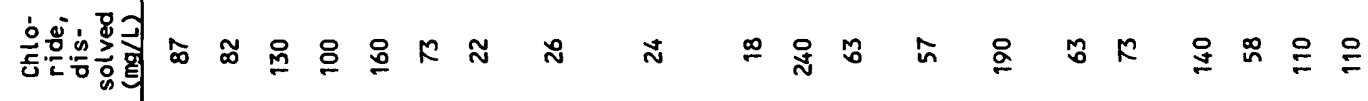

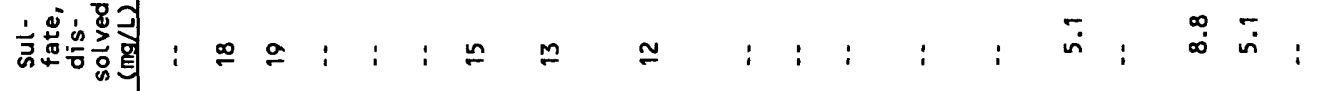

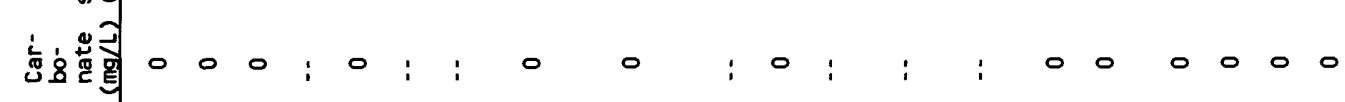

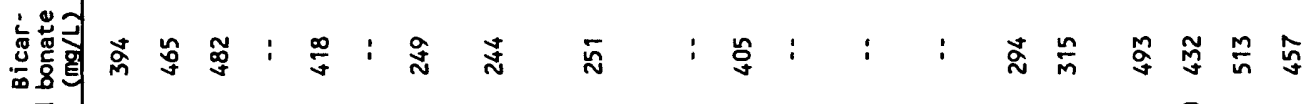

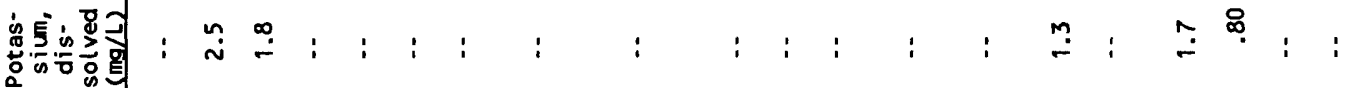

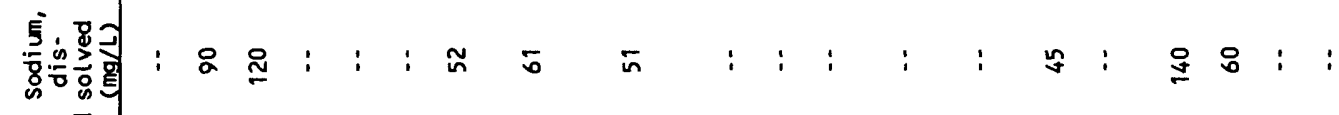

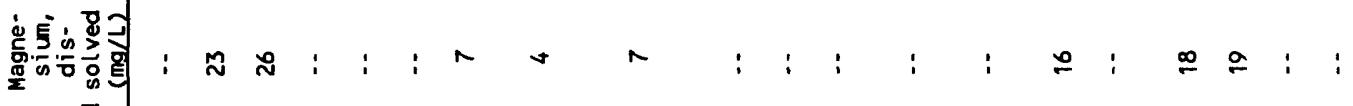

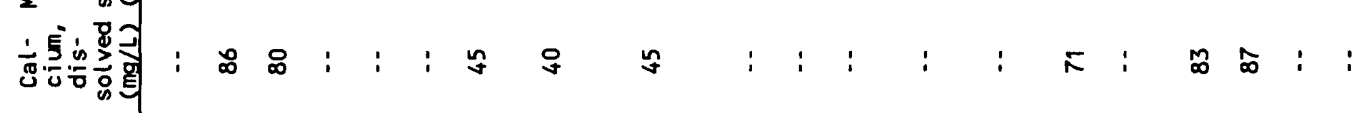

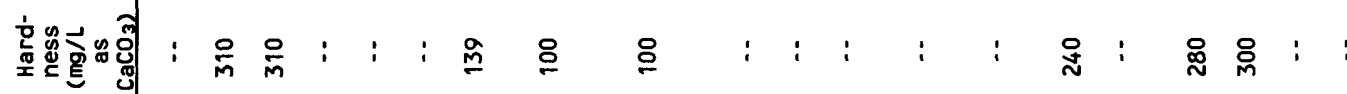

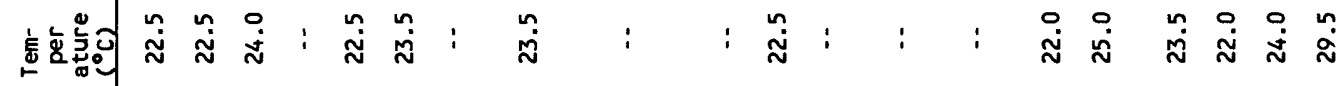

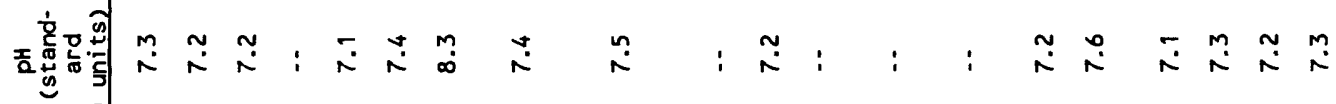

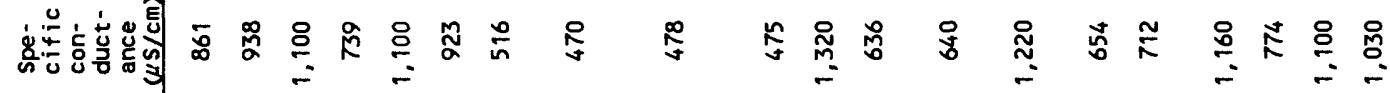

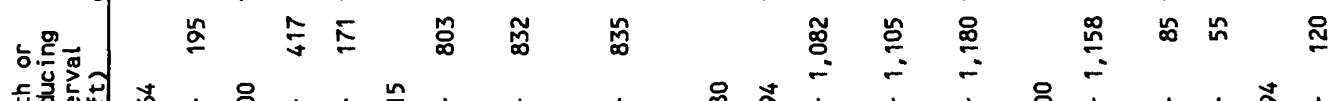

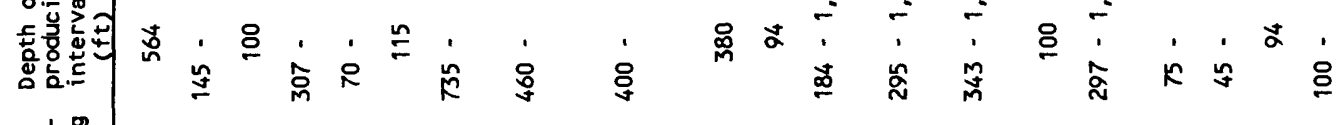

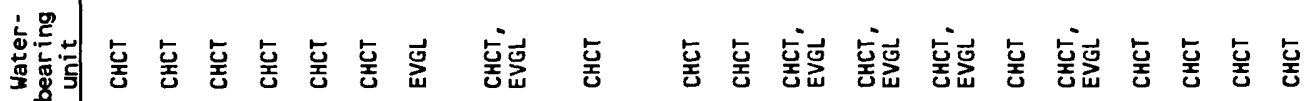

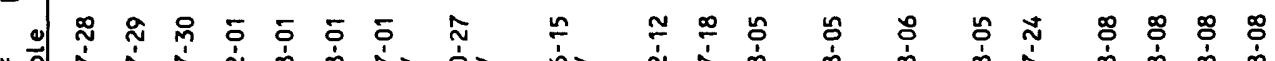

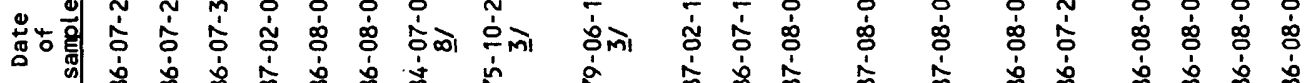

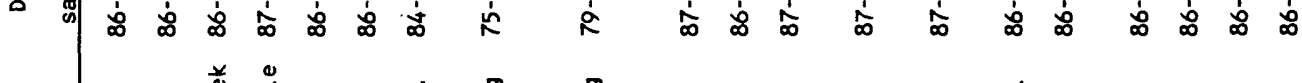

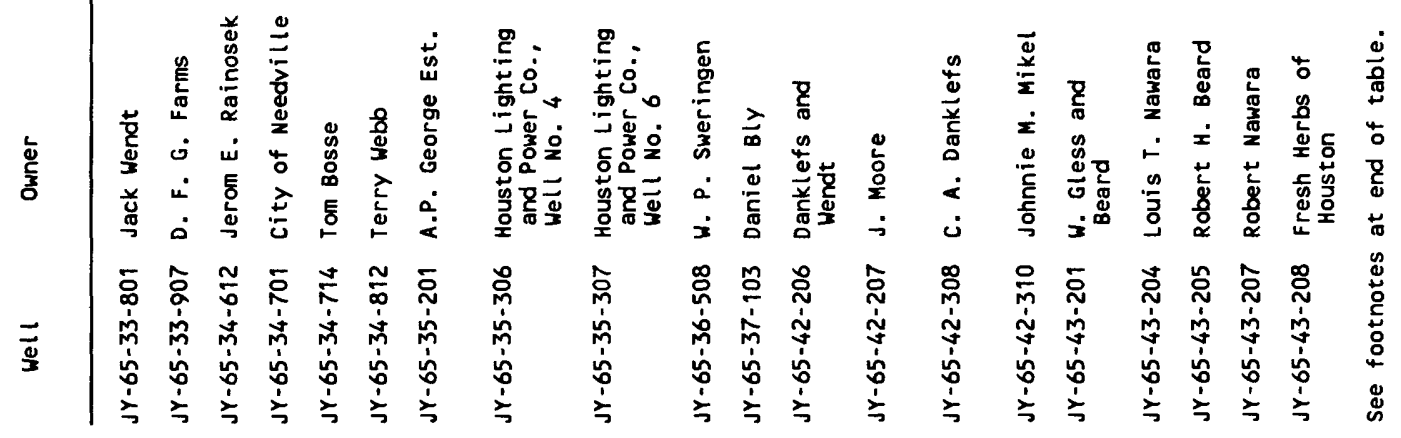




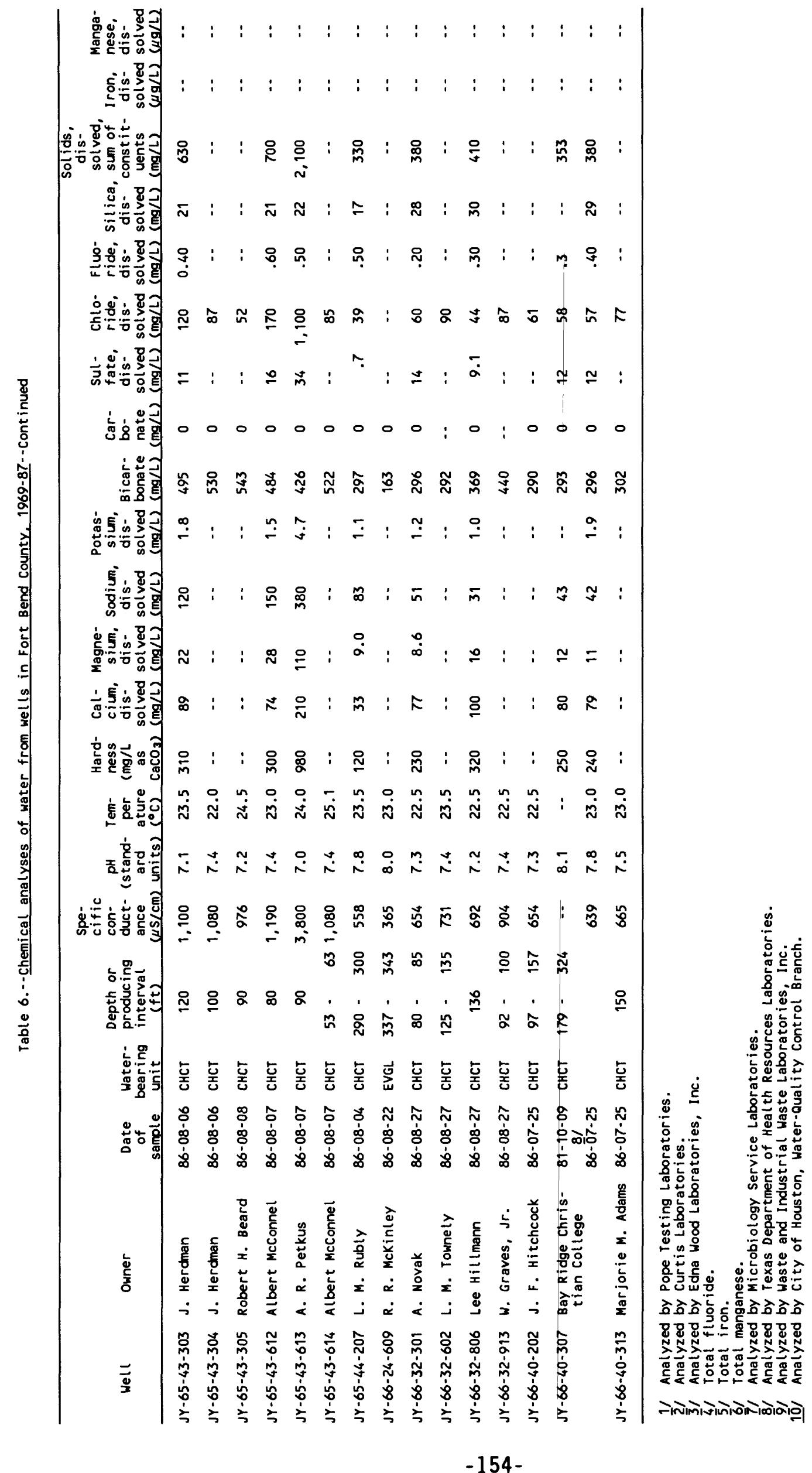


Table 7.-- statistical summary of the concentrations of major dissolved ions, and the values of specific conductance, hardness, and sodium-adsorption ratio

[ $\mu \mathrm{s} / \mathrm{cm}$, microsiemens per centimeter at $25^{\circ}$ Celsius; mg/L, milligrams per liter]

\begin{tabular}{|c|c|c|c|c|c|c|c|c|c|c|}
\hline Aquifer & $\begin{array}{l}\text { Spe- } \\
\text { cific } \\
\text { con- } \\
\text { duct- } \\
\text { ance } \\
(\mu s / \mathrm{cm})\end{array}$ & $\begin{array}{l}\text { Hard- } \\
\text { ness } \\
\text { (mg/L } \\
\text { CaCO3) }\end{array}$ & $\begin{array}{l}\text { Calcium } \\
(\mathrm{mg} / \mathrm{L})\end{array}$ & $\begin{array}{l}\text { Magne- } \\
\text { sium } \\
\text { (mg/L) }\end{array}$ & $\begin{array}{l}\text { Sodium } \\
\text { ad- } \\
\text { sorp- } \\
\text { tion } \\
\text { ratio }\end{array}$ & $\begin{array}{l}\text { Sodium } \\
+ \text { potas- } \\
\text { sium } \\
(\mathrm{mg} / \mathrm{L})\end{array}$ & $\begin{array}{l}\text { Bicar- } \\
\text { bonate } \\
\text { (mg/L) }\end{array}$ & $\begin{array}{c}\text { sul - } \\
\text { fate } \\
\text { (mg/L) }\end{array}$ & $\begin{array}{l}\text { Chlo- } \\
\text { ride } \\
\text { (mg/L) }\end{array}$ & $\begin{array}{l}\text { Solids } \\
\text { dis- } \\
\text { solvec } \\
\text { (mg/L) }\end{array}$ \\
\hline \multicolumn{11}{|c|}{ Upper unit of the Chicot } \\
\hline $\begin{array}{l}\text { Number of } \\
\text { analyses }\end{array}$ & 99 & 133 & 114 & 113 & 65 & 95 & 184 & 151 & 186 & 144 \\
\hline $\begin{array}{l}\text { Smallest } \\
\text { value }\end{array}$ & 534.0 & 22.0 & 5.0 & 6.1 & 0.5 & 13.0 & 160.0 & 2.0 & 12.0 & 264.0 \\
\hline $\begin{array}{l}\text { Largest } \\
\text { value }\end{array}$ & $1,470.0$ & 580.0 & 156.0 & 66.0 & 20.7 & 224.0 & 698.0 & 80.0 & 250.0 & 882.0 \\
\hline Mean & 901.2 & 271.6 & 78.2 & 22.9 & 2.45 & 81.7 & 398.7 & 16.9 & 79.7 & $\begin{array}{l}496.4 \\
475.0\end{array}$ \\
\hline $\begin{array}{l}\text { Medi an } \\
\text { Standard }\end{array}$ & 880.0 & 258.0 & 78.0 & 21.0 & 2.0 & 80.0 & 403.0 & 10.0 & 71.5 & 475.0 \\
\hline deviation & 99.0 & 108.3 & 29.9 & 10.7 & 2.6 & 36.0 & 101.3 & 15.8 & 43.8 & 131.1 \\
\hline
\end{tabular}

Lower unit of the Chicot

$\begin{array}{ccccccccccc}\begin{array}{c}\text { Number of } \\ \text { analyses }\end{array} & 107 & 98 & 70 & 70 & 61 & 49 & 109 & 94 & 119 & 62 \\ \begin{array}{c}\text { Smallest } \\ \text { value }\end{array} & 365.0 & 56.0 & 15.0 & 3.1 & 0.7 & 23.0 & 132.0 & 0.7 & 21.5 & 220.0 \\ \begin{array}{c}\text { Largest } \\ \text { value }\end{array} & 1,360.0 & 330.0 & 106.0 & 37.0 & 9.0 & 194.0 & 475.0 & 60.0 & 230.0 & 650.0 \\ \begin{array}{l}\text { Mean } \\ \begin{array}{l}\text { Median } \\ \text { Standard }\end{array}\end{array} & 651.2 & 178.6 & 60.9 & 11.2 & 2.0 & 69.5 & 268.0 & 12.7 & 65.2 & 377.2 \\ \quad \text { deviation } & 365.0 & 64.9 & 21.1 & 5.7 & 1.6 & 43.2 & 65.9 & 8.8 & 40.4 & 101.7\end{array}$

\section{Evangel ine}

\begin{tabular}{ccccccccccc}
$\begin{array}{c}\text { Number of } \\
\text { analyses }\end{array}$ & 42 & 35 & 34 & 34 & 22 & 27 & 40 & 35 & 44 & 32 \\
$\begin{array}{c}\text { Smallest } \\
\text { value }\end{array}$ & 441.0 & 11.0 & 3.5 & 0.5 & 1.4 & 37.0 & 124.0 & 0.8 & 23.0 & 270.0 \\
$\begin{array}{c}\text { Largest } \\
\text { value }\end{array}$ & $1,090.0$ & 240.0 & 54.0 & 11.0 & 31.8 & 247.0 & 342.0 & 19.0 & 173.0 & 682.0 \\
$\begin{array}{l}\text { Mean } \\
\begin{array}{l}\text { Median } \\
\text { Standard } \\
\text { deviation }\end{array}\end{array}$ & 580.9 & 110.0 & 31.9 & 5.6 & 5.8 & 84.7 & 244.2 & 11.6 & 57.9 & 345.2 \\
& 441.0 & 49.6 & 13.6 & 2.2 & 6.4 & 43.4 & 38.4 & 5.3 & 36.3 & 94.0 \\
\hline
\end{tabular}

NOTE: Table includes data presented by Wesselman (1972, table 7). 